University of Tennessee Health Science Center

UTHSC Digital Commons

\title{
Cephalometric Evaluation of One-Phase and Two-Phase Treatment Alternatives in Matched Class li Subjects
}

Fredrick Jerome Burr

University of Tennessee Health Science Center

Follow this and additional works at: https://dc.uthsc.edu/dissertations

Part of the Orthodontics and Orthodontology Commons

\section{Recommended Citation}

Burr, Fredrick Jerome, "Cephalometric Evaluation of One-Phase and Two-Phase Treatment Alternatives in Matched Class li Subjects" (2007). Theses and Dissertations (ETD). Paper 35. http://dx.doi.org/10.21007/ etd.cghs.2007.0040.

This Thesis is brought to you for free and open access by the College of Graduate Health Sciences at UTHSC Digital Commons. It has been accepted for inclusion in Theses and Dissertations (ETD) by an authorized administrator of UTHSC Digital Commons. For more information, please contact jwelch30@uthsc.edu. 


\title{
Cephalometric Evaluation of One-Phase and Two-Phase Treatment Alternatives in Matched Class li Subjects
}

\author{
Abstract \\ "Early" or two-phase orthodontic treatment of Class II malocclusions is a highly debated topic in the \\ orthodontic literature. We report here on a retrospective cephalometric study of patients with Class II, \\ division 1 malocclusions. One group consisted of 32 consecutively treated patients who received "early" \\ treatment with a Fränkel II appliance followed by treatment with full fixed appliance. These subjects were \\ "matched" by sex and cephalometric value, to a subject treated with standard edgewise appliances in a \\ single phase. Matching criteria focused on the bony facial characteristics, notably ANB, NAP, Y Axis, \\ AOBO, FMA, overjet, and overbite. The question was whether the cephalometric results at the end of \\ treatment were comparable. Two-phase subjects were treated on average 2 years longer than the one- \\ phase edgewise group. Neither integumental variable ( $Z$ angle, E plane) differed statistically. Two of eight \\ skeletal variables differed statistically, namely ANB and FMA. ANB was on average $1.3^{\circ}$ smaller in the \\ two-phase group, while the FMA was on average $1.8^{\circ}$ steeper in the one-phase group. Four of the eleven \\ dental variables differed statistically between the two groups. These variables were related to incisor \\ position. Overall, the incisors in both treatment groups proclined during treatment. The two-phase \\ subjects had a lower rate of premolar extraction (3\% vs. $56 \%$ ) than the one-phase subjects. Similar \\ skeletodental endpoints were achieved regardless of treatment protocol; indicating the treatments can be \\ viewed as equivalent approaches to a common problem.

\section{Document Type} \\ Thesis

\section{Degree Name} \\ Master of Dental Science (MDS)

\section{Program} \\ Orthodontics

\section{Research Advisor} \\ Edward F. Harris, PhD

\section{Keywords} \\ Orthodontics, Class II malocclusion, Functional appliance, Mandibular retrognathia, Occlusion, Esthetics, \\ Facial growth

\section{Subject Categories} \\ Dentistry | Medicine and Health Sciences | Orthodontics and Orthodontology
}




\title{
CEPHALOMETRIC EVALUATION OF ONE-PHASE AND \\ TWO-PHASE TREATMENT ALTERNATIVES IN \\ MATCHED CLASS II SUBJECTS
}

\author{
A Thesis \\ Presented for \\ The Graduate Studies Council \\ The University of Tennessee \\ Health Science Center
}

\author{
In Partial Fulfillment \\ Of the Requirements for the Degree \\ Master of Dental Science \\ From The University of Tennessee
}

By

Frederick Jerome Burr, D.D.S.

May 2007 
Copyright ( 92007 by Frederick Jerome Burr All rights reserved 


\section{ACKNOWLEDGEMENTS}

I would like to thank my wife, Amanda, and my son, Elliott, for their never ending and loving support during my residency and during this thesis. I would also like to thank Dr. Edward Harris for all of his support and leadership during the writing of this manuscript. Without the help of Dr. Harris, this project would not be possible. I would also like to thank Dr. Marlin Grimes and Dr. Joe Wasson for serving on my committee. I appreciate their support, direction, and friendship. 


\begin{abstract}
"Early" or two-phase orthodontic treatment of Class II malocclusions is a highly debated topic in the orthodontic literature. We report here on a retrospective cephalometric study of patients with Class II, division 1 malocclusions. One group consisted of 32 consecutively treated patients who received "early" treatment with a Fränkel II appliance followed by treatment with full fixed appliance. These subjects were "matched" by sex and cephalometric value, to a subject treated with standard edgewise appliances in a single phase. Matching criteria focused on the bony facial characteristics, notably ANB, NAP, Y Axis, AOBO, FMA, overjet, and overbite. The question was whether the cephalometric results at the end of treatment were comparable. Two-phase subjects were treated on average 2 years longer than the one-phase edgewise group. Neither integumental variable ( $Z$ angle, E plane) differed statistically. Two of eight skeletal variables differed statistically, namely ANB and FMA. ANB was on average $1.3^{\circ}$ smaller in the two-phase group, while the FMA was on average $1.8^{\circ}$ steeper in the one-phase group. Four of the eleven dental variables differed statistically between the two groups. These variables were related to incisor position. Overall, the incisors in both treatment groups proclined during treatment. The two-phase subjects had a lower rate of premolar
\end{abstract}


extraction (3\% vs. 56\%) than the one-phase subjects. Similar skeletodental endpoints were achieved regardless of treatment protocol; indicating the treatments can be viewed as equivalent approaches to a common problem. 
$\begin{array}{lll}\text { Chapter Page } & \end{array}$

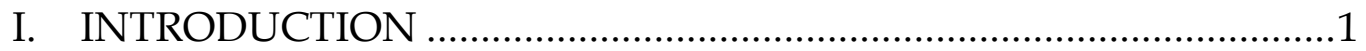

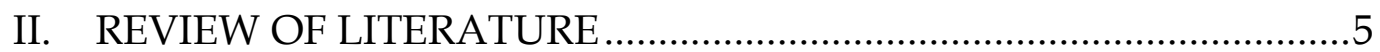

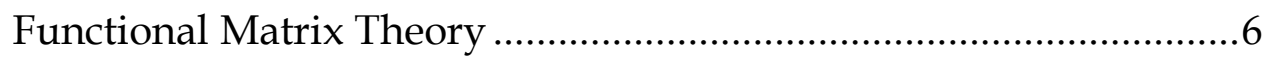

Application of the Functional Matrix Theory

to Functional Jaw Orthopedics .......................................................8

Indications for Fränkel Treatment.................................................13

Mandibular Deficiencies ...........................................................15

Condylar Cartilage and Mandibular Growth ...................................15

Treatment Effects Produced by the Fränkel Appliance......................17

Characterizing the Class II Malocclusion ...........................................28

Occlusal Development in Untreated Class II Malocclusions............30

Comparison of One- and Two-Phase Treatments..............................33

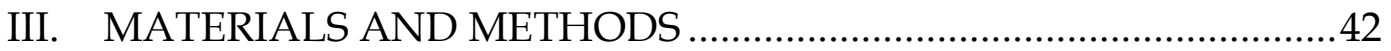

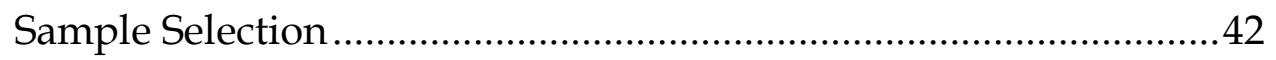

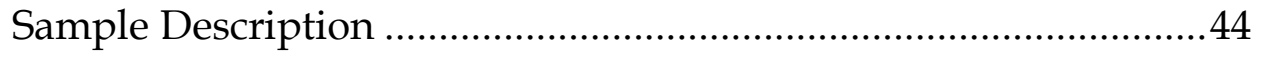

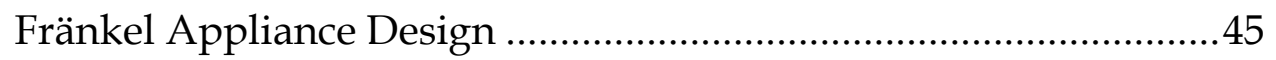

Cephalometric Analysis ....................................................................5

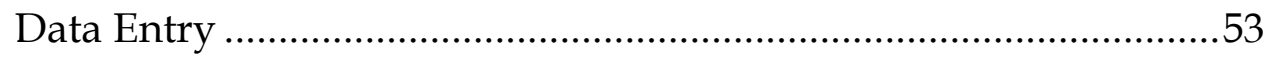


Statistical Analysis

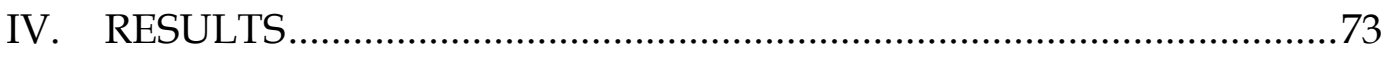

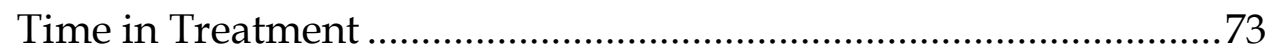

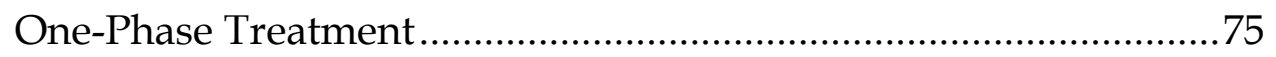

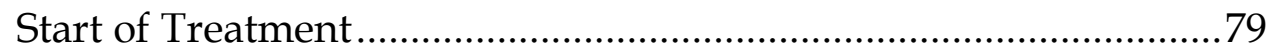

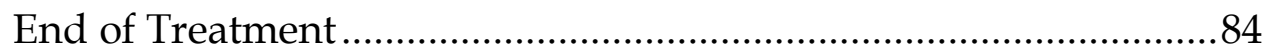

Patterns of Skeletodental Changes.................................................. 87

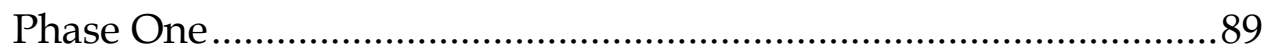

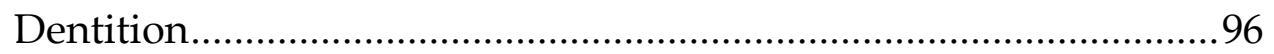

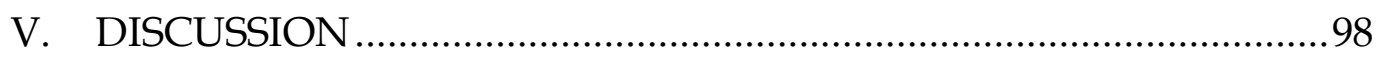

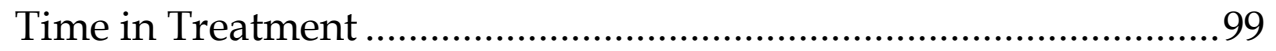

Patterns of Skeletodental and Dentoalveolar Change .....................101

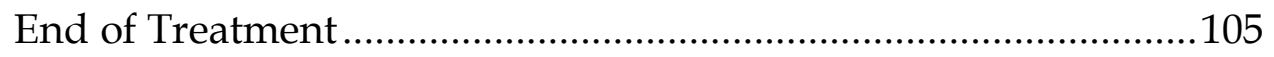

VI. SUMMARY AND CONCLUSIONS ...............................................107

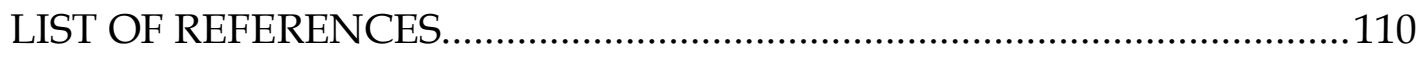

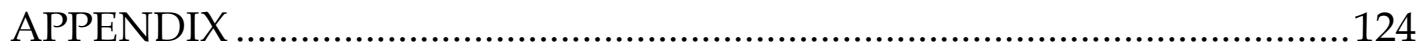

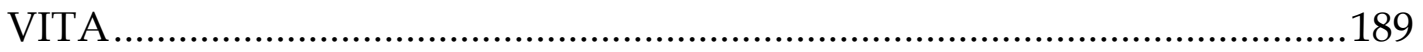

vii 


\section{LIST OF TABLES}

Table

1. Comparison of chronological age and time in treatment between the two treatment groups

2. Status of the one-phase sample at the two examinations, with tests for in-treatment changes

3. Descriptive statistics and inferential comparisons between starting conditions.

4. Comparison of skeletodental means between the two treatment groups at the end of treatment

5. Descriptive statistics for the two-phase sample $(n=32)$ at the three cephalometric examinations

6. Tests of change within each phase and comparison between changes of the two-phase group 


\section{LIST OF FIGURES}

Figure

1. Cephalometric diagram showing locations of the skeletodental landmarks to be used in this study....

2. Schematic tracing of a lateral cephalogram showing construction of the SNA angle.

3. Schematic tracing of a lateral cephalogram showing construction of the SNB angle

4. Schematic tracing of a lateral cephalogram showing construction of the angle ANB

5. Schematic tracing of a lateral cephalogram showing construction of the angle of convexity (NAP)

6. Schematic of the method of measuring the AOBO discrepancy.

7. Schematic tracing of a lateral cephalogram showing construction of FMA

8. Schematic tracing of a lateral cephalogram showing construction of the angle formed by the intersection of Frankfort Horizontal and Se-Na line

9. Schematic tracing of a lateral cephalogram showing construction of Down's Facial Angle.

10. Schematic tracing of a lateral cephalogram showing construction of the angle of the mandibular incisor and the mandibular plane (IMPA)

11. Schematic tracing of a lateral cephalogram showing construction of FMIA measurement 
12. Schematic tracing of a lateral cephalogram showing construction of the angle between the upper incisor with the lower incisor (i.e., interincisal angle)

13. Schematic tracing of overbite and overjet measurements................66

14. Schematic tracing of a lateral cephalogram showing construction of the $\mathrm{Y}$ axis angle

15. Schematic tracing of a lateral cephalogram showing construction of the Down's occlusal plane angle

16. Schematic tracing of a lateral cephalogram showing construction of the Condylion-B Point

17. Schematic tracing of a lateral cephalogram showing construction of the Condylion to Gnathion. 


\section{CHAPTER I}

\section{INTRODUCTION}

The prevalence of Class II malocclusions among children in the United States is approximately 15\% of all malocclusions (Kelly and Harvey 1977). Although Class II malocclusions are common, the optimal age for treatment has been controversial. One approach used today is to begin treatment during the preadolescent years (i.e., the mixed dentition) with limited treatment goals. This early treatment typically is followed by a second treatment phase with broader goals in adolescence; after the eruption of the permanent dentition. The common alternative approach is to initiate treatment in the early complete permanent dentition and forego the early treatment phase altogether. It has been estimated that at least one third of all children in orthodontic treatment are in a two-phase regimen (Gianelly 1995).

There has been considerable discussion in the orthodontic literature regarding the biological and clinical advantages and disadvantages of early intervention. Some have argued that early treatment has no long-term advantage (Tulloch et al. 2004). Others argue that early treatment may reduce treatment time and reduce the complexity of the second phase of treatment (Dugoni 1996). Reported benefits of early treatment include the ability to intercept and/or reduce dentoalveolar, skeletal, and neuromuscular 
abnormalities prior to the eruption of the permanent dentition (McNamara and Brudon 1993). Moyers (1988) suggests that early treatment of Class II malocclusions may harness normal growth to gain skeletal correction before the malocclusion becomes more severe, and he notes that compliance often is higher in younger patients. Other reported benefits include: reduced need for extraction, better treatment results, less potential for damage such as tooth fracture prior to treatment, and improved patient self esteem (Bishara et al. 1998). Opponents of two-phase treatment in Class II malocclusions contend that early treatment produces no reduction in the average time a child is in fixed appliances nor does it reduce the proportion of complex treatments involving extractions or orthognathic surgery (Tulloch et al. 2004).

In cases of Class II malocclusions with mandibular deficiencies, functional appliances often are used to stimulate mandibular growth. The notion that functional appliances can "stimulate" mandibular growth has been controversial. Björk (1951) and Nelson et al. (1993) concluded that functional appliances contribute little to final mandibular size. In contrast, authors such as Bolmgreen and Mishiri (1986), Mills (1991), Ghafari et al., (1998), and Illing et al. (1998) concluded that the use of functional appliances significantly enhances mandibular growth. Other research indicates that the treatment effects of functional appliances are restricted to dentoalveolar compensations (Chadwick et al. 2001). 
Of principal concern is the benefit of early Class II treatment in the mixed dentition compared with treatment started in the early permanent dentition. That is, gained with regard to treatment outcomes when using a two-phase treatment protocol compared to one-phase? A recent study on this question concluded that there is no difference in orthodontic outcomes between subjects treated in two phases compared to those treated in a single phase (Tulloch et al. 1998).

In a randomized clinical trial subjects are not allocated according to who may respond favorably to a certain treatment modality based on clinical evaluation (e.g., Smith and Pell 2003); rather, they are perforce randomly assigned to the treatment protocols. Although allocation bias is curtailed, the ability to choose treatment modalities based on clinical evaluation also is eliminated. Subjects that may respond favorably to early treatment may not be assigned to the treatment modality best suited for them.

The primary aim of the present study is to gain substantive information through cephalometric analysis on the efficacy of Fränkel II treatment in the early mixed dentition followed by subsequent full appliance treatment. The design used in the present thesis was to match each Fränkel-treated case, by sex and cephalometric values, to a corresponding one-phase edgewise-treated case. The matched edgewise sample receiving fixed appliance treatment in a single phase was used as a comparison to determine which patients responded 
favorably to early treatment and which would be equally served with full appliances treatment alone. 


\section{CHAPTER II}

\section{REVIEW OF LITERATURE}

The eponym “Fränkel Appliance” refers to Rolf Fränkel. His investigations into the etiology and treatment of dentoskeletal malocclusions in young children were the impetus for his development of the removable "function corrector." Prior to the 1970s, the widely accepted view of craniofacial growth was restrictive in that it was believed that the pattern of growth was established by 3 months of age and was unalterable thereafter (Brodie 1941). With numerous subsequent clinical studies, it became apparent that this narrow view was contraindicated by the skeletal changes that could be brought about by orthodontic treatment. Observed clinical changes were more compatible with Melvin Moss' functional matrix model (e.g., Moss 1968). In fact, the theoretical basis for Fränkel's appliance is founded in the functional matrix concept of Moss (e.g., Moss 1962), who, in turn, had expanded on the work of Van der Klaauw (1946). Throughout the inception, development, modification and clinical use of the appliance, Fränkel consistently stressed the theoretical basis of his appliance. 


\section{Functional Matrix Theory}

The functional matrix model, as postulated by Moss (e.g., 1962, 1968, 1969), has contributed greatly to the level of understanding of growth and development. Although certain aspects of the model have been disputed, the basic principles set forth in the functional matrix model have provided a viable interpretive framework for many of the problems associated with the understanding of craniofacial development.

Moss's theory was influenced by the ideas of Van der Klaauw (1946). Van der Klaauw asserted that the skull was made up of units, the size, shape, and position of which are determined by their functions. Using the basic concepts of Van der Klaauw and combining these with his own research, Moss postulated his functional matrix model.

The genetic predetermination of sutural growth (and, thus, bone growth) had been a dominant theory up to Moss's time. This concept adhered to the notion that within each suture was the genetic information that would determine the amount of growth occurring at the site of that suture (Sicher 1952). The suture was considered to be a growth center (Koski 1968). Moss argued, instead, that the suture was a growth site and the determinate of growth was the functional matrix enveloping the suture and adjacent bones (Moss 1954). 
According to Moss (1968), a functional cranial component consists of the tissues (hard and soft) and spaces that are necessary to carry out a specific function. Moss viewed the head as a structure that performs several functions. Each functional component is composed of two parts: (1) the functional matrix that consists of the soft tissue and spaces that perform a particular function and (2) the skeletal unit that is the hard tissue existing strictly in response to biomechanical demands for support, attachment, and protection of the functional matrix. Bone assumes the appropriate size and shape that best enables the soft tissues to perform their function. Moss' contention is that there is no need for genetic information in the skeletal unit because the functional matrix is endowed with the appropriate information for the initiation and direction of skeletal growth.

Moss (1969) conceptualized functional matrices as being of two sorts. The periosteal matrix is usually of the type that affects a portion of bone (termed a microskeletal unit). As an example, the temporalis muscle is responsible for the development of the coronoid process of the mandible. The capsular matrix, on the other hand, involves masses or spaces. Usually several different bones are involved in performing a particular function in a capsular matrix. As one example, the function of respiration depends on adequate spaces (nasal, pharyngeal, etc.) that in turn are enveloped by capsules of soft 
tissue that determine the development and morphology of more than one surrounding skeletal unit. The overall complex is termed a macroskeletal unit.

\section{Application of the Functional Matrix Theory}

$\underline{\text { to Functional Jaw Orthopedics }}$

Fränkel emphasized throughout his publications that Moss' model cannot be clinically adapted without modifications. Fränkel described how the theory applies to his own treatment by elaborating on four topics. First Fränkel reasoned that growth of related organs and tissues (i.e., a functional matrix) carrying out specific functions induces the growth and development of adjacent skeletal tissues (Fränkel 1980). An example is the function of the lateral ptyergoid muscle that protrudes the mandible. Increased growth due to hyperfunction of this muscle induces compensatory cartilage formation in the condyle (Fränkel 1980; Stokli and Willert 1971; Petrovic et al. 1975). Secondly, he reasoned that the size, shape, position, and maintenance of skeletal units are a response to the biomechanical demands for protection and support (Fränkel 1980). Fränkel reasoned that, by using "proper" orofacial orthopedics, aberrant muscular forces in the orofacial complex are changed, thereby correcting unfavorable growth forces. Fränkel cautioned that this concept has been badly misinterpreted. By altering the aberrant inductive mechanisms one will not get more than the pre-programmed genetic potential growth, but he does stress 
that, by creating a favorable growth environment, this growth potential will be more closely attained. He has argued that most abnormal skeletal patterns are not inherited. There is a predetermined tendency for disproportionate development; however, in a developing deformity, environmental factors also are involved. By disproportionate development Fränkel is implying that jaw growth is unbalanced and may cause either the maxilla or mandible to be retrusive or protrusive relative to one another. He stated, "A fundamental aim of orofacial orthopedics is to prevent adverse environmental factors from influencing the developmental course, thus providing the fullest accomplishment of a particular pattern in a given case" (Fränkel 1980:45).

Fränkel's clinical concept, like that of Moss, implies that "function dictates form "and "muscle is dominant over bone." Much orthodontic treatment with fixed appliances, other tooth-borne functional appliances, and surgical techniques embrace the scenario that, by mechanically altering function, muscle form and surrounding soft tissues will adapt (Fränkel 1980, 1966).

Fränkel stated that, "the musculature and nervous system develop in accordance with a fixed genetic plan and their anatomical shape and position are genetically predestined" (Fränkel 1974:382). The mechanical influences created by the functional matrix have the quality of genetic information, and, 
thus, skeletal tissue is being primarily shaped by "normal" function secondary to an intrinsically functioning matrix.

It is informative to propose a hypothetical situation involving the interruption of normal nasorespiratory function by an obstruction, such as hypertrophied adenoids. The functional matrix of respiration (the collection of muscles, other tissues and spaces that perform proper breathing) is altered by the nervous system, because inspiration is necessary for survival. The orofacial capsule can no longer undergo normal operation for the creation of an oral seal. The lips are required to be apart for survival and the closely interlaced facial musculature now responds to an abnormal triggering mechanism. The entire functional matrix for respiration undergoes change. Muscles now act to lower the mandible to facilitate oral inspiration. In so doing the growth of the mandible is altered and compensatory secondary cartilage formation at the condyle allows for the temporomandibular joint to continue functioning. The tongue assumes a more inferior and anterior position. With these several changes in the functional matrix, secondary to interruption of normal function, there are related bony changes of the supporting units for respiration. The alveolar arches are restricted or expanded according to the pressures of the change in tongue position and of the orofacial musculature. The size, shape, and position of the mandible are changed due to the mechanical forces acting on it. 
Recalling the concepts of the functional matrix model, Fränkel's claims for his therapy with the function corrector would be of benefit for the situation just hypothesized. Assuming that the etiology of the problem (nasal airway obstruction) is resolved, insertion of the appliance with its buccal and labial acrylic shields would interrupt abnormal muscle function. This is done by the shields facilitating normal mandibular position. The muscles are trained to function normally. So, if Fränkel's claims are correct, the appliance would inhibit the negative effect of improper function and allow alveolar bone and the teeth to reestablish proper development. The device will then retrain the musculature to function properly, according to Fränkel, which is necessary for a stable result. Therefore, a fundamental aim of functional therapy is to "reestablish functional performance as provided for in the genetic plan. This will allow for optimum morphogenetic development" (Fränkel 1980:112).

Fränkel stated that functionally adapted skeletal structures have the capacity for adaptive and compensatory growth at all sites where cells differentiate into osteoblasts and osteoclasts and that growth and development of various structures are largely controlled by genetic factors that predetermine the kind and range of the related functions (Fränkel 1980). Fränkel did not deny the influence of hereditary factors, especially on musculature and neural elements. However, he contended that there is, to a limited extent, an ability to influence the biomechanical inductive mechanisms themselves. Fränkel argued 
that a properly made appliance is able to stimulate morphology. The appliance induces the soft tissues to function properly and, hence, good skeletal growth is achieved.

Fränkel also stated that, "the functioning spaces as a factor of the functional matrix play an important role in craniofacial morphogenesis" (Fränkel 1980). He implies that a space per se does not constitute a dynamic factor. The only way the size of a space can be changed is from the size of the cranial components or outside pressures. This pertains to the oral cavity in that the muscular walls of the nasal and oral cavity can change their shape and size.

Another factor determining the size of the oral cavity is mandibular position. Fränkel stated that the primary factor for mandibular position is the maintenance of an adequate airway. Without adaptive, compensatory growth at the condyles, a functionally adaptive mandible would not be present (Fränkel 1974).

After adapting the functional matrix theory to the oral cavity, Fränkel claimed that the real problem is to design and construct an exercise device (i.e., orthodontic appliance) that directly interferes with the maladapted functional environment. The two main tasks of the appliance are (1) to prevent faulty muscular function and (2) to stimulate normal performance; it must act to retrain the musculature. By requiring that muscles function in normal posture, a more normal development of the dental and skeletal structures is attained. It 
is important to remember that the presence of teeth is necessary for the formation and function of alveolar bone. So, it is argued, if teeth are allowed to erupt without the inhibition of outside pressures (i.e., pressures of the lips, cheeks, and tongue), then it is more likely that a well rounded, uncrowded dental arch will develop. It can be argued that if the mandible is allowed to develop without abnormal posturing due to inadequate nasopharyngeal space, a more balanced predetermined genetic profile can be achieved.

\section{$\underline{\text { Indications for Fränkel Treatment }}$}

The Fränkel appliance is different from other functional appliances in that it uses the oral vestibule as its operational base. The configuration of the design is proposed to achieve alveolar and skeletal changes in a manner different from other functional appliances. Most functional appliances are tooth-borne or tooth-moving orthodontic appliances. The Fränkel appliance exerts its effects by withholding muscular pressure from the developing jaw and dentoalveolar areas, allowing changes in sagittal, transverse, and vertical dimensions. The appliance may be thought of as a deficiency appliance that stimulates underdeveloped structures rather than retarding overdeveloped structures.

There are several types of Fränkel appliances or "function regulators." Uses of the Fränkel I and II are similar, but the Fränkel II is the basic appliance 
of choice in cases of retropositioned mandibles (McNamara and Brudon 1993). Generally speaking, the Fränkel I is used when an anterior openbite is noted. The Fränkel II has been suggested for the following cases: (1) Class II skeletal crowding, (2) Class I skeletal relationships with crowding for width and development, (3) maxillary and mandibular anterior teeth normal to maxilla and mandible, (4) lower face height normal to less than normal, (5) crossbites, (6) a deep bite relationship (Diers 1998). The Fränkel III is used in Class III malocclusions, while the Fränkel IV typically is used in cases of skeletal openbites, steep mandibular plane angles, and excessive lower facial heights (Diers 1998).

The Fränkel appliance is indicated when it can work in concert with ongoing growth. The Fränkel appliance is of optimum use during seven to twelve years of age (Fränkel 1966). The four major claims made for the Fränkel appliance are: (1) stimulation of mandibular growth, (2) increased development of the alveolus in the anteroposterior and transverse dimensions, (3) "normalized" muscle function resulting in a better soft tissue profile, and (4) retardation of maxillary growth. Skeletal jaw deficiencies, dental crowding, abnormal muscle functions, openbites and crossbites are all within the realm of treatment proposed for the Fränkel appliance. 


\section{Mandibular Deficiencies}

Maxillary and mandibular differences result from some unknown combination of genetic and environmental forces. Whatever the cause, it is important to know the extent to which mandibular growth can be stimulated. Fränkel (1980) does not focus attention on stimulation of mandibular growth at the condyle. He insisted that with mandibular retrognathism, the key problem is whether the protractor muscle group can be trained so that a new mandibular postural performance pattern can be established. He favored gradual anterior repositioning of the mandible so the musculature is not overstrained.

Proponents of tooth-borne functional appliances (e.g., activators, bionators) approach skeletal deficiencies from a different perspective. These latter appliances are aimed at correcting skeletal form and this encourages the soft tissues to adapt. No direct attempt is made first to interrupt abnormal muscle function. This different approach is in opposition to that of the Fränkel functional corrector (Fränkel 1980).

\section{Condylar Cartilage and Mandibular Growth}

Correction of mandibular retrognathia by increased condylar growth due to hyperpropulsion of the mandible has been investigated. These studies vary in the manner in which the mandible was anteriorly positioned; some 
appliances were removable, while others were fixed. The histological composition of the temporomandibular joint have been shown, both in laboratory and clinical studies, to be susceptible to local extrinsic factors. Condylar cartilage of the mandible is unique in several respects. Whereas the primary effectors of growth of the epiphyseal cartilages of long bones, nasal cartilage, and cranial base synchondroses are growth hormone and somatomedin, condylar cartilage is to a great degree regulated by extrinsic local factors (particularly tension of the lateral ptyregoid muscle), as well as growth hormone (Koski 1977; Petrovic et al. 1974). Structurally, condylar cartilage consists of three basic zones. The articular zone is fibrous connective tissue and is located at the most superior aspect of the condyle. Internal to the connective tissue layer is the proliferative zone where appositional growth occurs by differentiation of mesenchymal cells into prechondroblasts, chondroblasts, and chondrocytes. The third, deepest zone is the hypertrophic zone, where chondrocytes are enlarged and bone formation occurs (Durkin 1972; McNamara et al. 1975).

Any type of appliance that causes chronic contraction of the lateral pterygoid muscle, reduction of the serial sacromeres, and, thus, actual shortening of the muscle has been shown by Petrovic (1975) to induce cellular proliferation in the prechondroblastic zone of the condylar cartilage. The "servosystem" theory is valuable here by way of interpretation (Petrovic et al. 
1981). Petrovic and coworkers stated that the maxilla, functioning normally, is under considerable genetic control and is a constantly changing reference point that the mandible must occlude with and react to. The optimal occlusal and cuspal relationship is a Class I molar relationship; Class II and Class III fall short of this. However, so long as any given occlusal relationship is not interrupted, skeletal and dental growth continues. Introduction of an appliance interrupts the occlusal relationship and, consequently, initiates a postural response. The lateral pterygoid contracts, protruding the mandible in search of a comfortable intercuspal relationship from which dentoskeletal growth can continue. This action of the lateral pterygoid appears to be a prime cause of condylar cartilage proliferation where chronic protrusion induces endochondral bone growth (Petrovic et al. 1975). It is generally understood that the condyle is not a "pacemaker" for growth of the mandible. Instead, the condyle possesses a "capacity for growth and remodeling in selective response to varied mandibular displacement and rotation. Very simply, it provides regional adaptive growth" (Enlow 1968:110).

Treatment Effects Produced by the Fränkel Appliance

Treatment effects produced by the Fränkel appliance can be arbitrarily divided into two types, namely the effects on dentoalveolar development and the effects on basal skeletal development. Fränkel (1966) stated that the 
developmental inhibition of the underlying skeletal structures that supports the teeth is causally related to perioral and buccal muscle function. By retraining the central nervous system, elimination of these aberrant perioral and buccal muscle activity may lead to a "full" development of the dental arches with fewer functional and morphological abnormalities. Fränkel theorized that his appliance is especially valuable when treatment begins in the early mixed dentition. The erupting tooth, in theory, acts as the "matrix" for alveolar growth (Fränkel 1974). Mosch (as cited by Fränkel, 1971) studied 400 patients treated only with the Fränkel appliance and observed that a spontaneous widening of the dental arches occurred routinely. Mosch also reported a mean increase in transpalatal width of over $4 \mathrm{~mm}$ in both the premolar and molar regions during a two year treatment time. Breidan and coauthors (1984) conducted a study of Fränkel patients in whom metallic implants had been placed in the maxilla and observed that most of the widening of the maxilla was due to deposition of new bone along the lateral border of the alveolus rather than due to increased growth at the midpalatal suture.

Creekmore and Radney (1983) conducted a study to evaluate: (1) the difference in mandibular growth response of Class II malocclusions compared to Class I malocclusions treated with Fränkel II therapy, (2) the influence of a functional orthopedic appliance (Fränkel II) on the growing facial complex compared to an untreated sample, (3) difference in Fränkel II therapy and 
edgewise therapy with extraoral traction, and (4) individual variations in growth and response to orthodontic therapy. A total of 9 Class I patients and 11 Class II patients were treated with the Fränkel appliance. Changes were evaluated cephalometrically and compared to an untreated Class II control sample of similar age and to an edgewise treated sample, all of whom were treated without extraction of permanent teeth. Compared to the untreated control sample, the combined Fränkel groups had significant mean increases in mandibular length (Co-Gn), lower facial height, retraction and elongation of maxillary incisors, retraction of maxillary molars, proclination of mandibular incisors, and increased vertical height of mandibular molars. Compared to Fränkel therapy, edgewise therapy had a greater retraction of the maxilla as seen by a mean reduction of SNA 1.7 degrees. Similarly, maxillary incisors were retracted without elongation (i.e., eruption). The mandibular incisors were retracted rather than protracted, and Pogonion came forward less than in the Fränkel group. Class II correction as measured by ANB reduction was similar for both treatment groups. The Fränkel group achieved this reduction mostly by an increase in SNB while the edgewise group correction resulted mostly from a reduction in SNA. As a result, Pogonion came forward more in the Fränkel group $(4.0 \mathrm{~mm})$ in comparison to the edgewise group $(2.5 \mathrm{~mm})$. The authors concluded that Fränkel appliance therapy was primarily orthodontic in nature with a small but significant orthopedic effect. 
Falck (1985) studied the long-term effect of Fränkel treatment on the skeletal and dental structures of the craniofacial complex. The study consisted of serial lateral cephalograms of 103 children with a Class II malocclusion associated with mandibular retrusion. Forty-five untreated subjects served as controls. The patients were followed from 7 years 6 months of age to 15 years and 5 months of age for the treated group, and 7 years 9 months of age to 15 years 1 month of age for the controls. Falck reported a mean forward movement of Pogonion of $14 \mathrm{~mm}$ in the treated group and $7.3 \mathrm{~mm}$ in the untreated group, disclosing a significant difference in mandibular growth increments.

Remmer and coauthors (1985) studied the effects of the activator, Fränkel II, and fixed appliance (edgewise) treatment in Class II malocclusion subjects. Lateral cephalograms taken before and after treatment were studied. Each group consisted of 25 nonextraction cases all of which were treated in a single phase. To make horizontal and vertical linear measurements, an $\mathrm{X}$ axis was established by joining the points Sella and Nasion. The Y axis was drawn through Sella perpendicular to the $\mathrm{X}$ axis. All horizontal measurements were made perpendicular to the $\mathrm{X}$ axis. A vertical measurement was made from Menton perpendicular to the $\mathrm{X}$ axis. Diagonal measurements were made from Sella to A Point and from Sella to Gnathion. Dial calipers were used to make the linear measurements. Relative to Sella, translation of the mandibular 
symphysis was the same in the three groups. There was a greater lingual tipping of the maxillary incisors in the Fränkel group compared to the fixed appliance group. Changes in soft tissue profile showed no statistically significant difference among the treatment groups at the end of treatment. There was no difference in the increase in total anterior face height, regardless of which treatment group was observed. In overview, the authors noted the remarkable similarity in the changes that occurred in the three groups.

Hamilton and coauthors (1987) evaluated the skeletal, dental, and condylar positional changes induced by Fränkel therapy. Pretreatment and posttreatment cephalometric, tomographic, and dental cast records of 25 consecutively treated cases were evaluated and comparisons were made to age and sex matched "normal" controls. It is important to clarify that these subjects were not matched with regards to severity of malocclusion. The Fränkel appliance appeared to have little effect on the anteroposterior growth pattern in that ANB was reduced on average only $0.4^{\circ}$ more than the untreated controls. After treatment, the Fränkel sample still displayed a significantly greater Class II skeletal relationship than the controls (ANB) with a deficient mandible (Cd to B Point). A statistically significant increase in mandibular plane angle was noted in the treatment group. Significant amounts of maxillary incisor retraction and mandibular incisor proclination were observed. Maxillary intermolar width along with the mandibular intercanine and intermolar widths 
were significantly increased during treatment an average of $2.3 \mathrm{~mm}, 1.3 \mathrm{~mm}$, and $1.0 \mathrm{~mm}$, respectively. No significant change in condylar position was noted. The results from this study indicate that the primary treatment effects of Fränkel therapy are dentoalveolar in nature.

McNamara et al. (1990) investigated the treatment effects produced by two types of functional appliances: (1) the Herbst appliance and (2) the Fränkel II "function regulator" (FR-II). Serial cephalometric radiographs from 45 patients treated with the acrylic splint Herbst appliance and 41 patients treated with the FR-II appliance were compared with serial radiographs of 21 untreated persons with Class II malocclusions. Treatment effects were identified through the use of cephalometric analysis consisting of 17 horizontal and vertical skeletal measures. Of the 4 maxillary skeletal measures considered, there was no significant difference between the Fränkel treatment group and the untreated controls. This finding suggests that treatment effects produced by Fränkel therapy are essentially dentoalveolar in nature.

Ghafari et al. (1998) conducted a prospective randomized clinical trial to evaluate the treatment of Class II, division 1 malocclusions in prepubertal children. A total of 63 subjects were evaluated in the study. All subjects had a Class II division, 1 malocclusion, a minimum ANB angle of $4.5^{\circ}$; they were between 7 and 13 years of age; and they had no prior orthodontic treatment. Facial and occlusal changes after treatment with either a headgear or a Fränkel 
function regulator were evaluated. Molar and canine relationships, overjet, intermolar and intercanine distances were measured from casts taken every 2 months. Cephalometric headfilms were taken annually and sagittal skeletal, sagittal dentoalveolar, and vertical skeletal measurements were taken. Results indicated that, on average, the headgear had a distal effect on the maxilla and first molars, but not the maxillary incisors. The function regulator also restrained growth of the maxilla but resulted in a retroclination of the maxillary incisors (decrease in Upper 1-NA angle of $5.22^{\circ}$ ), a more forward position of the mandible (increase in Cd-Pg $5.02 \mathrm{~mm}$ ), and a proclination of the mandibular incisors (increase in Lower 1-NB of $3.10^{\circ}$ ). The effect of both appliances on mandibular length were similar. Intermolar width increased in both groups, but more with Fränkel appliance use. Maxillary and mandibular intermolar widths increased $2.9 \mathrm{~mm}$ and $1.0 \mathrm{~mm}$ in the Fränkel group and $1.3 \mathrm{~mm}$ and 0.40 $\mathrm{mm}$ in the headgear group. Overjet correction was greater with the Fränkel appliance, presumably due to an increased retroclination of the maxillary incisors.

Toth and McNamara (1999) conducted a retrospective cephalometric study that compared the treatment effects produced by the twin-block appliance, Fränkel appliance, and an untreated control sample. A total of 40 Class II patients were observed in each group. Treatment effects of the Fränkel sample included a small but statistically significant decrease in the distance 
from Nasion-perpendicular to Point A in both treatment groups. However, overall maxillary skeletal effects of both functional appliance treatments were only minor. Mean mandibular length as measured from Condylion to Gnathion increased $2.7 \mathrm{~mm}$ in the control group and $4.6 \mathrm{~mm}$ in the Fränkel group, indicating an increase in mandiular length. In addition, a statistically significant increase in the Articulare to Gnathion measurement occurred in the Fränkel sample compared to the untreated sample. This observation of increased mandibular growth, however, did not produce significant increases in the SNB angle or the Nasion-perpendicular to Pogonion measure. The increase in mandibular length was not translated into an advancement of the chin point in the Fränkel group. The ANB angle was reduced $1.1^{\circ}$ in the Fränkel patients and remained unchanged in the control patients. Similarly, the Wits appraisal decreased by $2.2 \mathrm{~mm}$ in the Fränkel sample, whereas there was only a minor change in the untreated sample.

Relative to controls both functional appliance treatments tended to produce increases in vertical facial measures. These increases were more pronounced in the twin-block patients. Fränkel subjects had significant increases in the Frankfort horizontal to occlusal plane $\left(+1^{\circ}\right)$, ANS to Menton (+2.1 mm), and Condylion to Gonion $(+2.9 \mathrm{~mm})$ measurements. Although a mean increase in lower anterior facial height (ANS to Menton) was observed in the Fränkel patients, there was no corresponding increase in the mandibular 
plane angle. Perhaps both anterior and posterior vertical facial dimensions increased, resulting in a stable mandibular plane angle. There was a slightly larger vertical movement of the mandibular molars in the Fränkel patients than in the controls. Lingual tipping of the maxillary incisors was noted in the Fränkel group $\left(-3.3^{\circ}\right)$ and some proclination of the mandibular incisors $\left(+1.0^{\circ}\right)$ occurred as well. The movement of the maxillary first molars in the Fränkel group was not statistically different from the controls. In conclusion, treatment effects of the Fränkel appliance produced minimal dentoalveolar changes and appeared to have primarily a skeletal effect. It has been hypothesized that tissue-borne appliances, such as the Fränkel, produce less dentoalveolar change than tooth-borne appliances like the Bionator, Herbst and twin-block (Fränkel 1980).

Almeida and coauthors (2002) evaluated the dentoalveolar and skeletal cephalometric changes produced by the Fränkel appliance in individuals with Class II, division 1 malocclusions. Lateral cephalograms of 44 patients (sexes combined) were divided in two groups of 22 each. A control group of untreated Class II children (mean age 8 years 7 months) was observed for 13 months and was used for comparison. The Fränkel group had an initial mean age of 9 years and was treated for a mean period of 17 months. Cephalometric radiographs were taken at the beginning and end of treatment. No significant change in maxillary growth was observed during the observation period in 
either group. However, a statistically significant increase in mandibular length (Cd-Gn) of $1.7 \mathrm{~mm}$ occurred in the Fränkel treatment group. Both groups showed a slight downward rotation of the palatal plane, indicating no difference in craniofacial growth direction. The Fränkel appliance produced a labial tipping of the lower incisors $\left(+2.0^{\circ}\right)$ and a lingual inclination of the upper incisors $\left(-4.8^{\circ}\right)$ as well as a significant increase in mandibular posterior dentoalveolar height. No significant decrease in ANB was observed for any group. In overview, the authors state, "The major treatment effects of bionator and FR-II appliances were dentoalveolar, with a smaller, but significant, skeletal effects" (Almeida et al. 2002:464).

Janson and coauthors (2003) conducted a cephalometric study of the treatment effects of the Fränkel II appliance. Subjects consisted of 18 patients (mean age 9 years 3 months) with Class II, division 1 malocclusions. The treated group was compared with a control group of 23 untreated individuals observed during the same age interval. Lateral cephalometric headfilms were obtained for the treated group at the beginning of treatment and after 28 months of treatment. The subjects in the control group belonged to a serial growth study in which lateral cephalometric head films were obtained annually from 4 to 18 years of age. Results dislosed no statistically significant influence on maxillary development since changes in maxillary position and effective length were similar for both groups. There was no statistical 
significant difference in the effective length of the mandible (Co-Gn).

However, changes in mandibular body length were statistically significant and $1.6 \mathrm{~mm}$ greater in the treated group. Changes in the angular maxillomandibular variables (ANB and NAP) did not present statistically significant differences between the two groups. However, the changes in the proportion between maxillary and mandibular effective lengths (Co-A and Cd-Gn) were statistically significant. There was no significant change in FMA between groups. No increase in lower anterior face height (LAFH) was observed in the treated group, presumably due to posterior bite opening induced by the construction bite (Fränkel and Fränkel 1989). The treated group had significant palatal plane tipping and a decrease in protrusion of the maxillary incisors compared with the control group. The upper incisor to Nasion-A Point measurement was reduced on average $8.0^{\circ}$ in the treated group. The dentoalveolar height as well as the anteroposterior position of the maxillary molars did not vary significantly between the two groups. The anteroposterior changes in the mandibular incisors revealed no significant difference between the groups. However, the appliance did not produce any change in maxillary development, in the growth pattern, or any improvement in the basal relationships. These negative results indicate that Class II correction is primarily dentoalveolar, with only a small participation of skeletal changes. 


\section{Characterizing the Class II Malocclusion}

In a sample of Class II individuals, Elsasser and Wylie (1943) observed that maxillary protrusion occurred in males while the maxilla was in a relatively neutral position in females. No difference was noted in maxillary molar positioning compared to a Class I control group. In addition, these investigators found the mandibular length to be within normal limits for males, while it was less than normal in females.

Renfroe (1948) studied facial patterns in Class II malocclusions and observed that the maxilla was generally in a retrusive position in both sexes with maxillary incisor protrusion and molar retrusion relative to a Class I sample. He noted, as did Henry (1957), that while some Class II individuals have a deficiency in mandibular size, others have well formed mandibles of normal size. However, these normal mandibles were in a retruded position due to the posterior position of the glenoid fossae. Renfroe concluded that the mandibles of Class II individuals were retrognathic relative to other craniofacial structures.

Through an investigation of Class II individuals, Riedel (1952) determined that the maxillary skeletal base was normally positioned in both sexes but with maxillary incisor protrusion. He also noted that the mandible was retrusive relative to the averages of Class I individuals. 
Henry (1957) developed a classification of Class II division 1

malocclusions. He selected his sample according to Angle's classification system, and categorized four groups for this malocclusion: (1) maxillary alveolar protrusion; (2) maxillary basal protrusion; (3) micromandible; and (4) mandibular retrusion. From cephalometric evaluation, Henry noted an increased mandibular plane angle in Class II cases compared to Class I norms, suggesting an increase in lower facial height.

In assessing a Class II sample, Hunter (1967) found the maxilla to be in a relatively neutral position, but with incisor protrusion. The mandibular skeletal position was retrognathic while the mandibular incisors were retruded. He also observed a slight increase in anterior facial height.

McNamara (1981) examined a series of Class II patients to determine the nature and frequency of specific contributing components. The study was a cross-sectional evaluation of the lateral cephalograms of 277 children ages 8 to 10 years old. From these records, he assessed maxillary and mandibular skeletal and dental relationships. The most common findings were an excessive lower facial height and mandibular retrusion.

Moyers and coworkers (1980) in a study of 697 North American white children divided Class II individuals into six horizontal and five vertical groups. Using a procedure of aborization, he established 15 subtypes of Class II malocclusions. Analysis of these subtypes revealed persistant skeletal 
characteristics for each group during intervals of growth. He concluded that $20 \%$ had a maxillary protrusion while over $50 \%$ had a mandibular retrusion with little, if any, maxillary protrusion.

\section{Occlusal Development in Untreated Class II Malocclusions}

An indication of how to treat a malocclusion may be gained by observing how it changes with time. White (1983) examined 34 cases who began with an end-end molar relationship in the early mixed dentition. Following these until the end of the mixed dentition, he observed that 24 developed a Class I molar relationship, while the other 10 developed Class II relationships. The maxillary molars moved mesially an average of $2.3 \mathrm{~mm}$, and the average mandibular increment was $1 \mathrm{~mm}$ in a mesial direction from the initial observation. These tooth movements worsened the molar relationships. White (1983) concluded that differential jaw growth was a more important factor in the process of molar adjustment than mesial drift of the molars.

Whitney (1983) evaluated an untreated longitudinal Class II sample and recognized eight groups within this type of malocclusion. The groups displayed an array of skeletal variations and severities of protrusiveness and retrusiveness of the skeletal base. A majority of the cases were mandibular retrusive. He noted a tendency for maxillary protrusion with a maxillary bony arch that was consistently longer than the mandibular corpus. The differential 
between the two arches increased with age, resulting in a progressive worsening of the Class II relationship. Behrents (1985) conducted a follow-up study of the same sample and found that, while growth continues into adulthood, existing maxillomandibular relationships are maintained in a fairly uniform manner with only small variations.

Byczeck, Ngan and Scheick (1997) compared skeletal growth changes between Class II, division 1 and Class I females between the ages of 7 and 14 years. Lateral cephalometric radiographs had been taken annually from age 5 through 17. They observed that the maxilla was no more protrusive in the Class II sample when compared with the Class I sample. In fact, there was a decrease in maxillary prognathism in Class II subjects during the pubertal growth period. The maxillo-mandibular skeletal difference (ANB) was significantly greater in the Class II sample at age 7 and did not improve with age; consequently, skeletal differences maintained a greater degree of facial convexity in the Class II subjects. The authors state that, "Those results suggest that the Class II skeletal growth pattern is established early and maintained throughout puberty unless altered by orthodontic intervention" (Byczeck et al. 1997).

Bacetti and others (1997) recorded occlusal features of the Class II malocclusion during the transition from the deciduous to the mixed dentition in untreated subjects. During the observation period, cephalometric changes 
consisted of significantly greater maxillary growth increments and smaller increases in mandibular dimensions in the Class II sample. In addition, they observed a downward and backward rotation of the mandible over time with a subsequent decrease in the gonial angle for the Class II subjects. They concluded, "all occlusal Class II features were maintained or became exaggerated during the transition to the mixed dentition" (Bacetti et al. 1997). These findings are similar to those found by Fröhlich (1961) who reported that no improvement of Class II occlusal relationship occurs from 5 to 12 years of ages and Arya and coworkers (1973) who observed that all patients presenting with a distal step relationship of the second deciduous molars exhibit a Class II relationship in the permanent dentition.

Bishara (1988) conducted a cross-sectional and longitudinal evaluation of the changes in mandibular length and maxillary-mandibular relationships in untreated Class II subjects from the deciduous to permanent dentition. These Class II samples were compared with matched "normal," untreated individuals. Longitudinal comparisons of growth profiles indicated that the growth trends were similar between the untreated Class II, division 1 subjects and normal subjects. There was no "self correction" with growth in the untreated Class II sample.

The aforementioned literature suggests that Class II dental malocclusions do not "self correct" or improve with time. If anything, they 
tend to worsen with time. This would permit subjects with similar pretreatment Class II malocclusions to be compared with one another regardless of the age at the start of treatment. In the current thesis, subjects of varying initial ages but similar skeletal and dental characteristics are compared to each other.

\section{Comparison of One- and Two-Phase Treatments}

A goal of "early" orthodontic treatment is to correct existing or developing skeletal, dentoalveolar, and muscular imbalances to improve the orofacial environment before the eruption of the permanent teeth is complete (McNamara and Brudon 1993). Anticipation is that early intervention may reduce the overall need for complex orthodontic treatment that may include permanent tooth extraction or orthognathic surgery. On the surface, this seems reasonable; it appears more logical to prevent an abnormality from occurring rather than waiting until it has developed fully. In a recent survey of the 159 Diplomates of the American Board of Orthodontics, participants were asked what they perceived to be the benefits of early treatment (Bishara et al. 1998). The most common responses were as follows: (1) greater ability to modify growth; (2) improved patient self-esteem and parental satisfaction, (3) better and more stable results, (4) less-extensive therapy is required later; and (5) reduced potential for tooth damage such as trauma, root resorption and 
decalcification. Responses of this survey were supported by a study by King and coworkers (1999) in which orthodontists perceived that subjects who had received phase 1 treatment had less complex malocclusions and lower treatment priority than subjects in an untreated control group.

Proponents of two-phase orthodontic treatment often contend that treatment in the late mixed dentition gives the clinician only one chance at correction, and if cooperation is poor the results may be unsatisfactory (Dugoni 1998). In addition, by delaying treatment many female patients may have passed the peak velocity of their skeletal growth and strategies aimed at growth modification may have reduced effectiveness. According to Dugoni (1998) benefits of treating Class II malocclusions in the early mixed dentition include the following: (1) reduced incidence of premolar extraction, (2) reduced need for surgical orthodontics, (3) better patient cooperation, (4) reduced incidence of root resorption, and (5) reduced incidence of ectopic cuspid eruptions. The Department of Orthodontics at the University of the Pacific is conducting a randomized retrospective study to evaluate treatment changes during early mixed dentition treatment (Dugoni 2006). The study sample was restricted to patients who originally were evaluated in the early and middle mixed dentition yielding three study groups: (1) delayed treatment (i.e., no treatment), (2) phase 1 treatment only, and (3) two-phase treatment. All subjects were treated by the same orthodontist. Preliminary analysis of the 
study indicates that approximately $42 \%$ of patients who received early treatment did not require a second phase of treatment. Subjects requiring full treatment and phase- 1 treatment had fewer visits, shorter treatment times, and lower fees. In addition, $82 \%$ of subjects in the early treatment group did not require extraction in the permanent dentition.

Another proposed benefit of early orthodontic intervention in Class II malocclusions is improved self-esteem. O'Brien and coworkers (2003) conducted a multicenter, randomized, controlled trial providing early functional appliance treatment for children aged 8 to 10 who presented with Class II, division 1 malocclusions. Comparisons were made to age and sex"matched" subjects who were untreated. The second phase of the study examined the psychosocial impact of early orthodontic treatment. A total of 89 subjects were allocated to a twin-block group and 87 to an untreated control group. Data were collected at baseline and 15 months later, at which time no subject was still wearing the twin-block appliance. A series of questionnaires were used to gather data concerning psychosocial effects of early orthodontic intervention. Results indicated that children who had received early treatment reported higher self concepts and more positive childhood experiences than the untreated controls.

However, not all clinicians agree, many preferring to wait until all the permanent teeth have erupted (excluding third molars) to start treatment. 
Opponents of two-phase treatment argue that there are few, if any, benefits that are unique to and dependent on earlier treatment. Gianelly (1995) contends that at least $90 \%$ of all growing patients can be treated successfully in only one phase by starting treatment in the late mixed dentition. Gianelly (1995) proposed that the other $10 \%$ of patients could benefit from immediate resolution of the problem such as those presenting with crossbites or Class III malocclusions. Opponents of two-phase treatment contend that patients probably only have a limited capacity to cooperate, and dual treatments that require two phases of compliance and retention may be more than patients can handle (Keeling et al. 1995; Berg 1979). We are unaware of other than anecdotal evidence for this claim, however.

Opponents also contend that there is no benefit concerning self-esteem and early Class II treatment. Dann and coworkers (1995) conducted research on children with Class II malocclusions concerning early treatment and its effect on self concept. The study consisted of 209 subjects whose overjet was greater than $4.5 \mathrm{~mm}$ and who were younger than 15 . Self concept was measured using the Piers-Harris children's self concept scale, which is an 80 item "forced" choice self report designed to quantitatively assess how children feel about themselves. Untreated controls were used for comparison. The results indicated that there was no change in mean self concept score in treated subjects, nor was there any association between reduction of Class II 
malocclusion features and and improved self concept. These findings suggest that children with Class II malocclusions do not generally present for treatment with low self concept and, on average, self concept does not improve during early orthodontic treatment.

Tulloch and coauthors (2004) conducted a randomized clinical trial of preadolescent (early) versus adolescent (later) treatment of children with severe Class II malocclusions. Severe malocclusions were those having an overjet greater than $7 \mathrm{~mm}$. A total of 166 children in the mixed dentition with Class II division 1 malocclusions were randomly assigned to one of three groups: (1) headgear, (2) bionator, or (3) no treatment (i.e., observation). After 15 months, records were taken and the groups pooled together and each child was randomly assigned to one of four orthodontists for treatment with traditional fixed appliances. Cephalometric radiographs were used to assess skeletal changes. The peer assessment rating (PAR) was used to rate alignment and occlusion. In the evaluation of Phase I treatment, statistically significant differences were observed between the treatment and observation groups although responses were widely variable. The change in jaw relationship (annualized reduction in ANB angle) was favorable in $76 \%$ of the headgear group, in $83 \%$ of the functional appliance, and in $31 \%$ of control (observation only) group. Evaluation of Phase II treatment evaluated whether these changes represented long term changes. Results from Phase II of the study indicated 
that the initial correction becomes overshadowed with time. Differences among groups with respect to skeletal relationship and PAR score were not statistically significant at the end of Phase II treatment. In addition, two-phase treatment appeared to be inefficient in that it did not reduce the average time a child spent in fixed appliances nor did it reduce the complexity of later treatment (i.e., need for extraction or orthognathic surgery). "During phase 2 of the trial, the advantage created during phase 1 of treatment in the two early treatment groups was lost, and, by the end of fixed appliance treatment, there was no significant difference between any of the three groups" (Tulloch et al. 2004:660).

O'Brien and coauthors (2003) evaluated the effectiveness of early orthodontic treatment with a twin-block functional appliance in a multicenter, randomized clinical trial. In comparison to the study conducted by Tulloch and coauthors (2004), this study used 14 hospital-based orthodontic specialists in the United Kingdom. Importantly, treatment was provided by many operators outside of a controlled university setting where treatment is carried out on selected populations. In overview, the authors wanted to see how effective early Class II treatment is in the "real world" of orthodontic practice outside dental schools. Children between the ages of 8 to 10 years with Class II, division 1 malocclusions $(\mathrm{n}=174)$ were randomly allocated to receive treatment with the Twin-block appliance or to an untreated control group. 
Data were collected at the start of the study and 15 months later. The results showed that treatment with the twin-block appliance reduced overjet, corrected molar relationship, and reduced the severity of the malocclusion. The majority of the correction was attributed to dentoalveolar changes and small amounts of favorable skeletal change. The study continued until the children had completed phase 2 treatment. Operators treated the children according to their normal treatment protocols. An aim of the study was to learn whether early treatment resulted in a reduced need for phase 2 treatment, and if differences in skeletal pattern or final dental occlusion were evident. At the end of phase 2 treatment, there was no difference between the patients who had early treatment and those who did not for any variable evaluated, and most treated subjects required a second phase of treatment ( $\mathrm{O}^{\prime}$ Brien et al. 2003). In conclusion, these findings agree with those of Tulloch and coauthors (2004) in that it appears that early orthodontic treatment for Class II malocclusions does not confer any advantage over a later single-phase treatment.

Breman and Pancherz et al. (2002) studied the efficiency of early and late Class II division 1 treatment. Efficiency was defined as a better result in a shorter time. Pretreatment and posttreatment dental casts of 204 patients in the early mixed $(n=54)$, late mixed $(n=104)$, and permanent $(n=40)$ dentition were evaluated according to the peer assessment rating (PAR). Results showed that treatment time and PAR scores decreased with increasing dental 
development, indicating that early Class II division 1 treatment is less efficient and less successful than a later one phase treatment.

Johnston and Livierates (1995) conducted a study comparing one-stage and two-stage nonextraction alternatives in Class II samples. For all subjects, the clinician's initial intention was nonextraction therapy, either in a one-stage full appliance or two-stage bionater and full appliance treatment. The study sample consisted of 47 one-stage patients and 49 two-stage patients, all treated by a single orthodontist. Comparisons between the two groups, were conducted by examining pretreatment and posttreatment lateral cephalograms. Both groups exhibited similar patterns of skeletal change that could not be distinguished from each other. Skeletal changes in both groups were largely responsible for molar and overjet corrections. The magnitude of differential jaw growth was greater in the two-phase group presumably because treatment started earlier and finished later.

Dolce et al. (2005) conducted a similar comparison and observed an early mandibular response in patients treated with a Bionator. The Bionator was originally developed by Wilhelm Balters $(1964,1973)$. The Bionator is often used to treat malocclusions characterized, at least in part, by mandibular deficiency. The Bionator produces a forward positioning of the mandible, promoting a new postural position of the mandible (McNamara and Brudon 1993). The data revealed that the sagittal jaw relationship improved 
significantly in both phase 1 treatment groups compared with the observation group. However, this initial mandibular response was not evident after both groups had received full appliance therapy. This study differed from previous studies in that it used centrographic analysis. Centrographic analysis is a visual analysis with no angles to measure or normative values to compare. Landmark position relative to an established reference plane allows the tracking of landmarks in horizontal and vertical planes. 


\section{CHAPTER III}

\section{MATERIALS AND METHODS}

\section{Sample Selection}

This present study is a retrospective analysis of cephalograms from patients with Class II, division 1 malocclusions at the start of treatment. These were 32 consecutively treated patients who received phase 1 (early) treatment with a Fränkel II appliance and later treatment with full fixed appliances. Cephalometric radiographs were available at T0 (start of treatment), T1 (end of phase 1 treatment), and T2 (end of treatment) for these subjects. Records were obtained from the office of Dr. Joe L. Wasson, Memphis, Tennessee, who had treated all of these cases. The control group consisted of a sample of subjects with similar Class II, division 1 malocclusions who were treated at the University of Tennessee, Department of Orthodontics, with fixed appliances only (Standard Edgewise).

Since phase 1 treatment usually begins at a younger age, the issue arose of how to match the two groups. All cases were American whites, and data collected included the sex, chronological age, duration of treatment, and the cephalometric status for each subject. The design was to match a one-phase orthodontic case, by sex and cephalometric values, to each of the 32 two-phase cases treated with the Fränkel II appliance. Matching criteria focused on the 
bony facial characteristics, notably ANB, NAP, Y axis, and AOBO. In addition, subjects were matched with regard to FMA, overjet and overbite. We collected subjects who had bilateral Class II division 1 (at least a half step) malocclusions. All subjects were treated to Class I canine relationships. For inclusion, the subjects had to have diagnostic pre- and posttreatment lateral cephalograms and documentation regarding age at start of treatment (T0) and at the end of treatment (T1). No other inclusion filter (e.g., outcome or "cooperation") was used. All too commonly in the orthodontic literature groups are labeled "matched" when, in fact, there merely have similar group characteristics. "Matched" is used in its correct statistical sense in the present study, where each individual in one (Fränkel) sample is matched demographically and cephalometrically to a control (edgewise) case. Consequently, the two sample sizes are directly comparable and, more importantly, repeated-measures statistical designs (Winer et al. 1991) can be used in place of croup comparison tests. A repeated-measures analysis of variance (or, equivalently, a paired ttest) matches each case from one group with a case from the second group, so the difference between the groups is tested as a function of the standard error of the mean difference. This measure of variability is always smaller than the more common group comparison t-test (or factorial ANOVA), so it is more efficient (i.e., more likely to discover a difference if one actually exists). In other 
words, a paired t-test is less likely to produce a type II statistical error (i.e., acceptance of a false null hypothesis).

\section{$\underline{\text { Sample Description }}$}

Dr. Joe L. Wasson, Memphis, Tennessee employs a three tier treatment strategy for Class II malocclusion treatment. Fränkel patients typically fall into the first tier. According to Dr. Wasson, patients that typically respond well to Fränkel appliance therapy often present with certain characteristics. They tend to be in the early mixed dentition (7 to 10 years of age), with high mandibular plane angles, openbite tendencies, and deleterious oral habits (e.g., tongue thrusts). In addition, these patients tend to have large overjets and constricted or underdeveloped dental arches. Treatment of these patients typically occurs in a two-phase regimen. The first phase includes Fränkel II wear, approximately 12 hours a day for approximately 1 year. Patients are seen every 2 months for evaluation with chair time being relatively short. In between phase 1 and phase 2 treatment, patients are instructed to continue Fränkel wear at night only for retention purposes. When the permanent canines and premolars emerge (excluding second and third molars) full appliances are placed.

The second tier of Dr. Wasson's treatment strategy is geared to patients who have Class II division 1 malocclusions. These patients tend to have low 
mandibular plane angles and deepbite tendencies. These patients are somewhat older than the Fränkel patients but are still in the mixed dentition $(8$ to 11 years of age). According to Dr. Wasson, these patients respond well to Bionator therapy.

The third treatment strategy is aimed at Class II patients in the early permanent dentition (11 to 14 years of age). This group of patients often is treated with fixed functional appliances such as the Herbst appliance and MARA (i.e., mandibular advancement repositioning appliance).

\section{Fränkel Appliance Design}

The appliance consists of an intricate configuration of wire onto which self-curing acrylic is applied, each part having a specific purpose (Fränkel and Fränkel 1980). The following description presents an outline of features and functions of this appliance. The reader is referred to the text by Graber and Neumann (1984) for actual construction of the appliance. The following items are a list of the components which make up the Fränkel II appliance:

Labial pad: This pad is positioned beneath the mentolabial fold on each side ofthe mandibular frenum. It does not contact the gingiva and must be constructed to allow constant, consistent opening and closing of the mandible without impinging on the loose or attached labial gingiva. The purpose of the pad is to interrupt abnormal muscle function in this 
region and, hopefully, initiate proper muscle function in the orofacial complex. Another benefit is the aid this shield provides in the attainment of an appropriate oral seal.

Lingual pad: This pad is positioned just beneath the crest of the gingiva on the lingual of the lower incisors. It does not rest upon the teeth and there is a slight relief from the attached gingiva. The purpose of this pad is to constantly "remind" the lateral pterygoid muscle to posture the mandible forward. If not, the mandible retrudes and contacts the acrylic of the pad. This forward posturing of the mandible is the result of a neuromuscular response precipitated by the lingual pad.

Buccal shields: These are positioned in the right and left sides of the oral vestibule. The shields extend from the most superior functional deflection of the unattached gingiva in the maxilla to the same position in the mandible. Mesiodistally, these pads extend from the distal aspects of the deciduous canine to the distal most aspect of the permanent first molar. Buccolingually, the shields stand away 3 millimeters from the maxilla, 1 to 2 millimeters from the mandible, and five to six millimeters from the teeth. The purpose of these shields is to prevent abnormal muscle function which would inhibit full development of the alveolar arches. Also, it is arguable that the shields 
stretch the periosteum in the buccal apical areas, thus enhancing bony deposition and broadening the alveolar arches (Fränkel 1975).

Maxillary labial wire: This wire $(0.036 ")$ strengthens and stabilizes the appliance. In rare incidences, it may be used to align an incisor.

Maxillary lingual wire: This wire $\left(0.035^{\prime \prime}\right)$ adds to the overall stability and isused to lock the appliance between the maxillary deciduous canine and deciduous first molar. Grooves are made in these teeth into which the lingual wire is fit to provide an anteriorposterior stop for the appliance.

Palatal wire: This wire's $\left(0.045^{\prime \prime}\right)$ primary purpose is to increases stability, but it also inhibits eruption of the maxillary molars by placing rests on the occlusal surfaces. Grooves are made interproximally between the deciduous second molar and permanent first molar to aid in retention.

Mandibular labial wire: This wire $\left(0.036^{\prime \prime}\right)$ is primarily used to hold the acrylic of the labial pads.

Mandibular lingual wires: These wires $\left(0.045^{\prime \prime}\right)$ also provide stability, but theymainly support the lingual shield. There are, in addition, certain smaller lingual wires that may be processed into the acrylic to apply labial pressure on the cingulum of the lower incisors to advance them labially or to impede eruption.

The appliance design affects the process of providing adequate space for the erupting permanent teeth. "Decrowding" is the process by which the 
permanent maxillary teeth, which are normally in a crowded position with their apical bases lingual to the deciduous counterparts, drift in a lateral direction while erupting (Dier 1998). Fränkel and coworkers (1987) noted that a different path of eruption occurred in the lower arches in patients treated with the Fränkel appliance than normally occurred in untreated individuals, with a less lingually-directed pattern of lower tooth eruption noted in the former. The process of providing adequate space for the erupting permanent teeth is controlled by genetic and environmental factors. With abnormal integumental forces, teeth are buccally restricted in their eruptive course, and crowding may result. Fränkel (1974) claimed that the buccal and labial shields of the function corrector remove oral pressures and allow for development of the alveolar arches. Elimination of cheek and lip forces with proper appliance construction and wear allows the intraoral forces, principally that of the tongue, to exert pressures that expand the arches facially. McDougall and coworkers (1982) compared serial dental casts from 60 patients treated with the Fränkel appliance to serial dental casts of 47 untreated individuals. The expansion was not limited to a particular region of either dental arch, although in absolute terms the greatest amount of expansion was in the premolar and molar regions. The average amount of expansion was $4-5 \mathrm{~mm}$ in the maxillary arch and 3-4 $\mathrm{mm}$ in the mandibular arch when compared to controls. In addition, Fränkel claimed that the buccal shields place tension on the vestibular 
tissues, which stimulates deposition of bone in the apical alveolar region and, thereby, displace muscle attachments buccally.

Fränkel (1974) contended that more than just facial muscle activity can adversely affect the natural decrowding process. The intraoral subatmospheric pressure that occurs when adequate anterior and posterior lip seal is present causes the lips and cheeks to "suck" in against the developing dentoalvolar structure. As the outer buccal shields of the Fränkel appliance are moved laterally, an increase in the "decrowding" mechanism of the dentoalveolar structures is proposed to occur. The proper timing of uncrowding was argued to be of utmost importance. Using Moss' model that the tooth is the functional matrix for alveolar growth, Fränkel reasons that in order to obtain maximum decrowding, the function corrector should be used during the period of active permanent tooth eruption: 7 to 12 years of age.

The Fränkel appliance design also aids in vertical discrepancies (open and closed). Interdental tongue posture is a compensatory or adaptive behavior intended to establish an anterior oral seal and tongue thrust, often labeled as an atypical or immature behavior pattern, should rather be considered as a proper compensatory physiological potential. According to Fränkel (1974), if a proper oral seal is obtained, there is no need for tongue exercises or devices to guide tongue function. After normal muscle function is achieved, the tongue will assume an appropriate position in the palate and the 
open bite will close. Fränkel admits that the most difficult task is to achieve good patient cooperation in performing lip seal exercises. However, once this is obtained and the labial pad of the FR-II is positioned beneath the mentolabial fold, the lower lip is more easily brought into contact with the upper lip. The neuromuscular functions of the external soft tissue capsule are forced to adapt to new conditions established by the vestibular shields when the lips are sealed. Tonic and motor aberrations of the buccinator and mentalis are redirected by the acrylic trainer leading to correction of structural and functional imbalance of the related muscles.

In the overclosed bite, the lip pad of the Fränkel appliance acts as a mechanical barrier to the invagination of the mentolabial fold, bringing the lips together and allowing the deep bite to normalize.

Moyers et al. (1980) stated that, "in the new orthodontics our emphasis may change to altering the conditions which determine the pattern of occlusion directly." The FR-II function corrector, when used on properly diagnosed children, would appear to fulfill this new perspective.

\section{Cephalometric Analysis}

Lateral cephalometric radiographs from three time points were used for the group being studied: (1) the pretreatment examination, (2) at completion of phase one treatment, and (3) at completion of full appliances. Since the control 
group was treated with fixed appliances in one phase, only pretreatment and post treatment radiographs were used for this group. A total of angular and linear variables were used in this study. The following alphabetical listing provides definitions of the cephalometric landmarks used in the study:

A Point A (Subspinale): the most posterior point on the exterior ventral curve of the maxilla between the anterior nasal spine and supradentale.

ANS Anterior Nasal Spine: the spinous process of the maxilla forming the most anterior projection of the floor of the nasal cavity.

B Supramentale: the most posterior point on the bony curvature of the mandible between Infradentale and Pogonion.

Ba Basion: the most inferior-posterior point in the midsagittal plane on the anterior margin of the foramen magnum at the base of the clivus.

$\mathrm{Cd}$ Condylion: the most superior and posterior point on the curvature of the capitulum of the condyle.

DOP Downs' Occlusal Plane: the line that vertically bisects incisal overbite and the most anterior occlusal contact of the maxillary and mandibular first molars (Downs 1948).

Gn Gnathion (anatomic): the most anterior-inferior point of the mandibular symphysis. 
Go Gonion (anatomic): the most posterior-inferior point on the gonial angle of the mandible.

$\mathrm{I}_{\mathrm{i}} \quad$ Incision inferius: the incisal tip of the most anterior mandibular central incisor.

$\mathrm{I}_{\mathrm{s}} \quad$ Incision superius: the incisal tip of the most anterior maxillary central incisor.

LIA Apex of mandibular central incisor: the apical end of the same mandibular central incisor used to locate $\mathrm{I}_{\mathrm{i}}$.

L6C L6 cusp: the mesial cusp tip of the mandibular first molar.

Me Menton: the most inferior point on the symphysis of the mandible.

Na Nasion: the anterior point of the intersection between the nasal and frontal bones.

Or Orbitale: the most inferior point on the lower margin of the bony orbit.

Pg Pogonion: the most anterior point on the anterior contour of the bony chin below B point and above Gnathion.

PNS Posterior Nasal Spine: the most posterior point at the midsagittal plane on the bony hard palate.

Po Porion: the midpoint on the superior aspect of the rim of the external auditory meatus.

Pt Pterygomaxillary fissure: the most superior-posterior point on the radiographic outlines of the pterygomaxillary fissure 
Se Sella turcica: the center of the hypophyseal fossa, determined by inspection.

U1A Apex of the maxillary central incisor: the apical end of the most anterior maxillary central incisor of the same tooth used to locate $\mathrm{I}_{\mathrm{s}}$.

U6C U6 cusp: the mesial cusp tip of the maxillary first molar.

\section{$\underline{\text { Data Entry }}$}

The cephalometric radiographs from all subjects were scanned using a UMAX Powerlook III flatbed scanner at $300 \mathrm{dpi}$ and 256 gray scale and were saved as TIFF files. The radiographs in TIFF format were imported into Dolphin Imaging ${ }^{\circledR} 10.0$ and traced using the program's digital cephalometric tracing. Prints of the cephalometric tracings of all the subjects used in the present study can be found in the Appendix. A custom analysis was created with the "custom analysis builder" function of Dolphin Imaging $10.0^{\circledR}$ for measurement of the 25 skelotodental angular and linear variables used in the present study. The measurements were exported from Dolphin Imaging $10.0^{\circledR}$ into Microsoft Excel ${ }^{\circledR} 2003$ and then into JMP ${ }^{\circledR}$ for statistical analysis.

\section{Statistical Analysis}

The skeletodental landmarks and angular measurements used in this study are illustrated in Figures 1 through 17. Data were collated into an Excel 


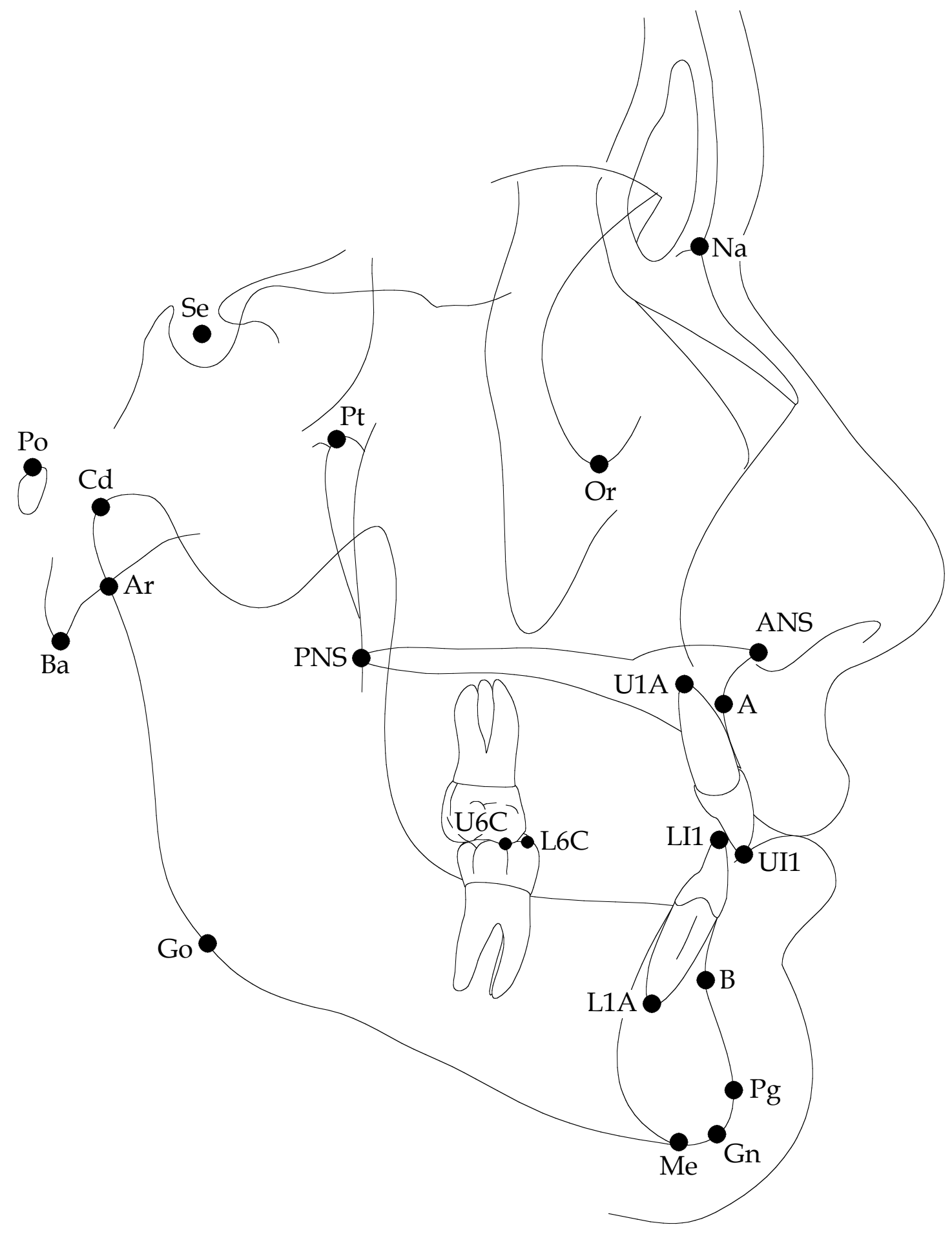

Figure 1: Cephalometric diagram showing locations of the skeletodental landmarks to be used in this study. 


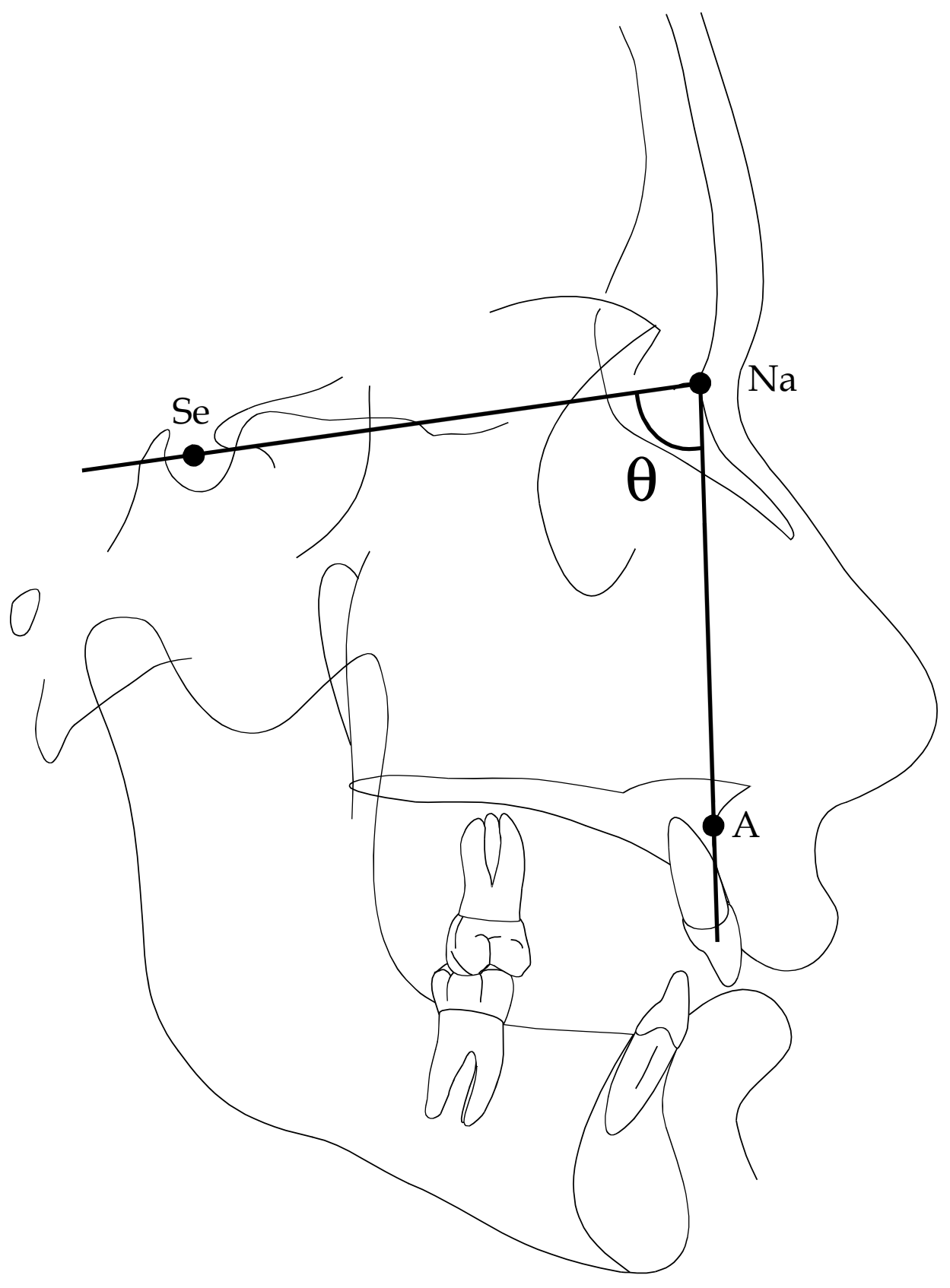

Figure 2: Schematic tracing of a lateral cephalogram showing construction of the SNA angle. 


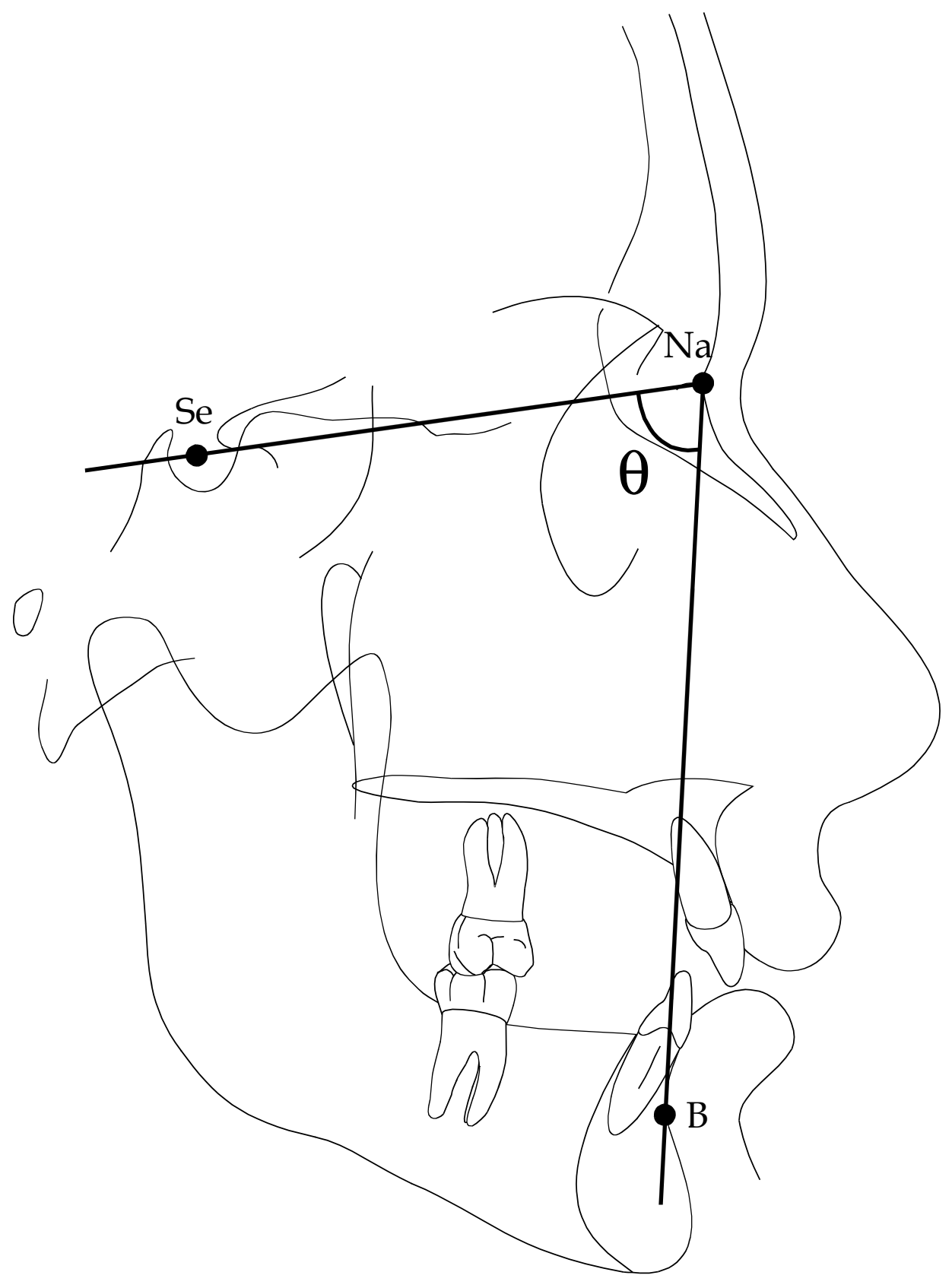

Figure 3: Schematic tracing of a lateral cephalogram showing construction of the SNB angle. 


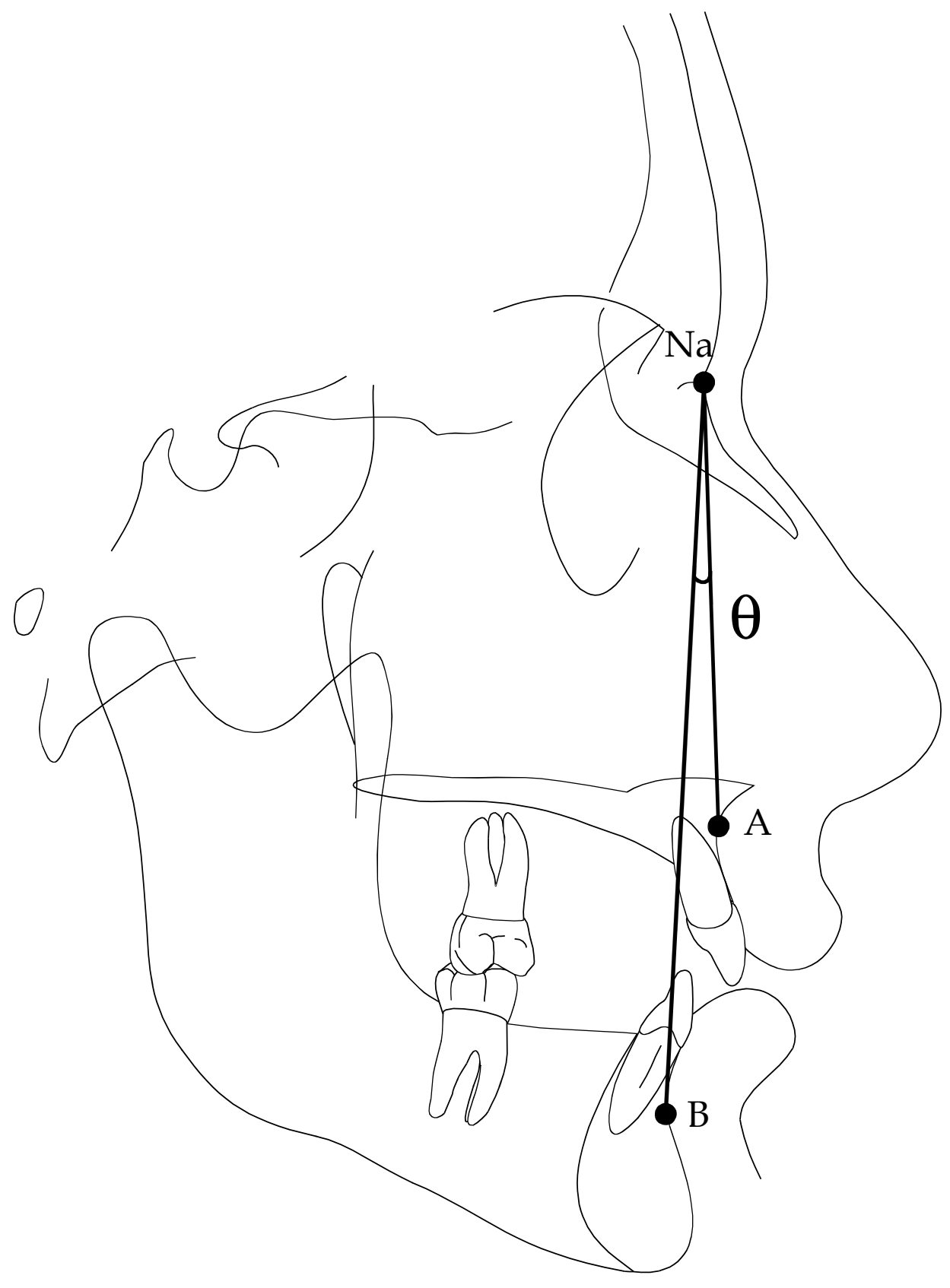

Figure 4: Schematic tracing of a lateral cephalogram showing construction of the angle ANB. 


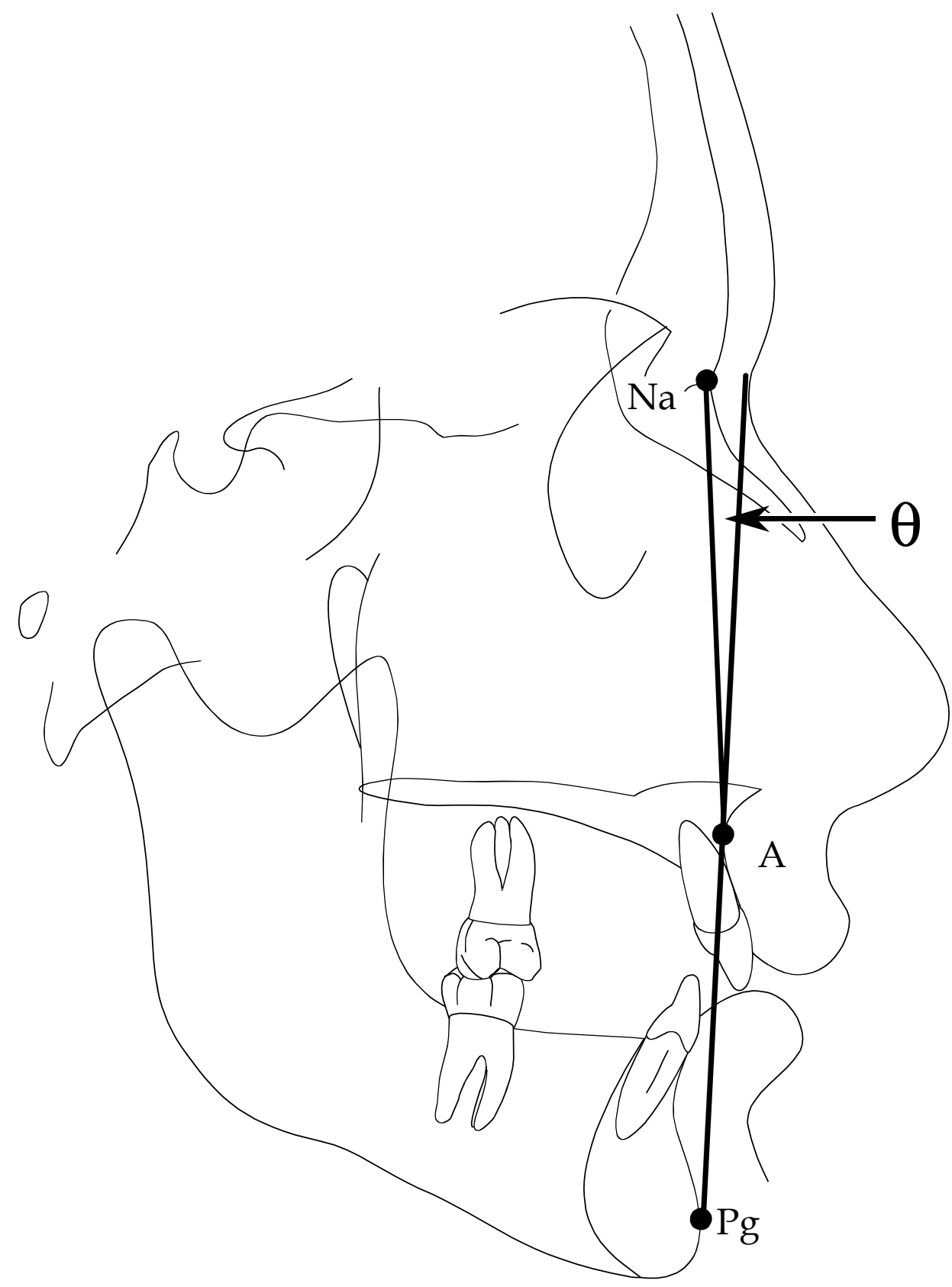

Figure 5: Schematic tracing of a lateral cephalogram showing construction of the angle of convexity (NAP). This is the superior-anterior angle at the intersection of the Nasian-A and A-Pogonion line. 


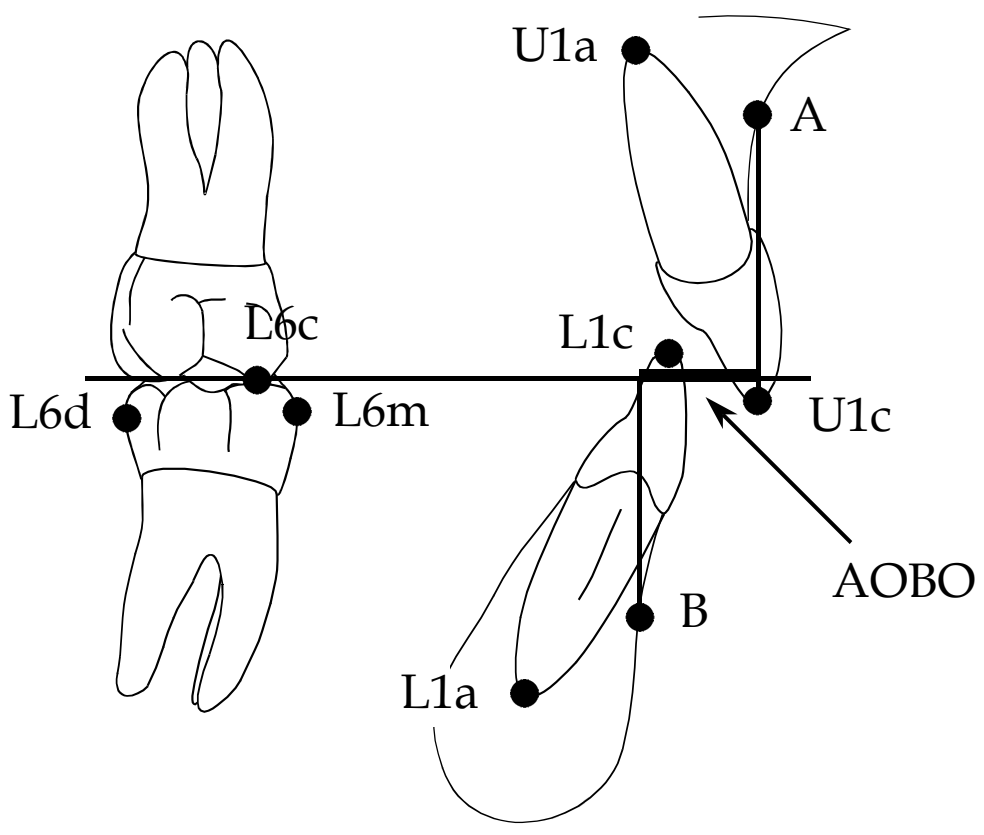

Figure 6: Schematic of the method of measuring the AOBO discrepancy. Point $A$ and Point $B$ are projected orthogonally onto the functional occlusal plane. $\mathrm{AOBO}$ is the millimetric distance between the projected line segment along the occlusal plane. 


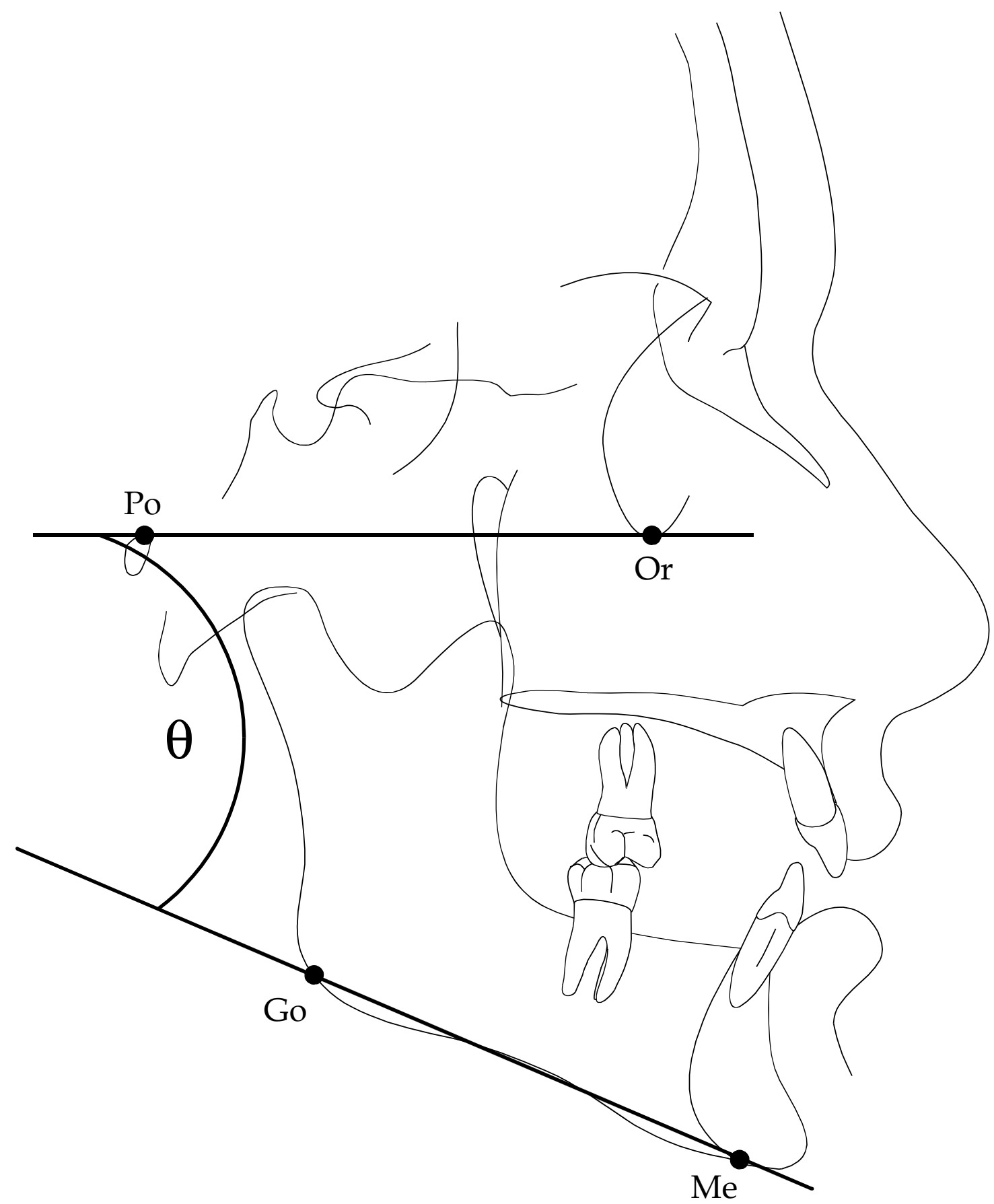

Figure 7: Schematic tracing of a lateral cephalogram showing construction of FMA. This is the angle formed by the intersection of Frankfort Horizontal and Gonion-Menton. 


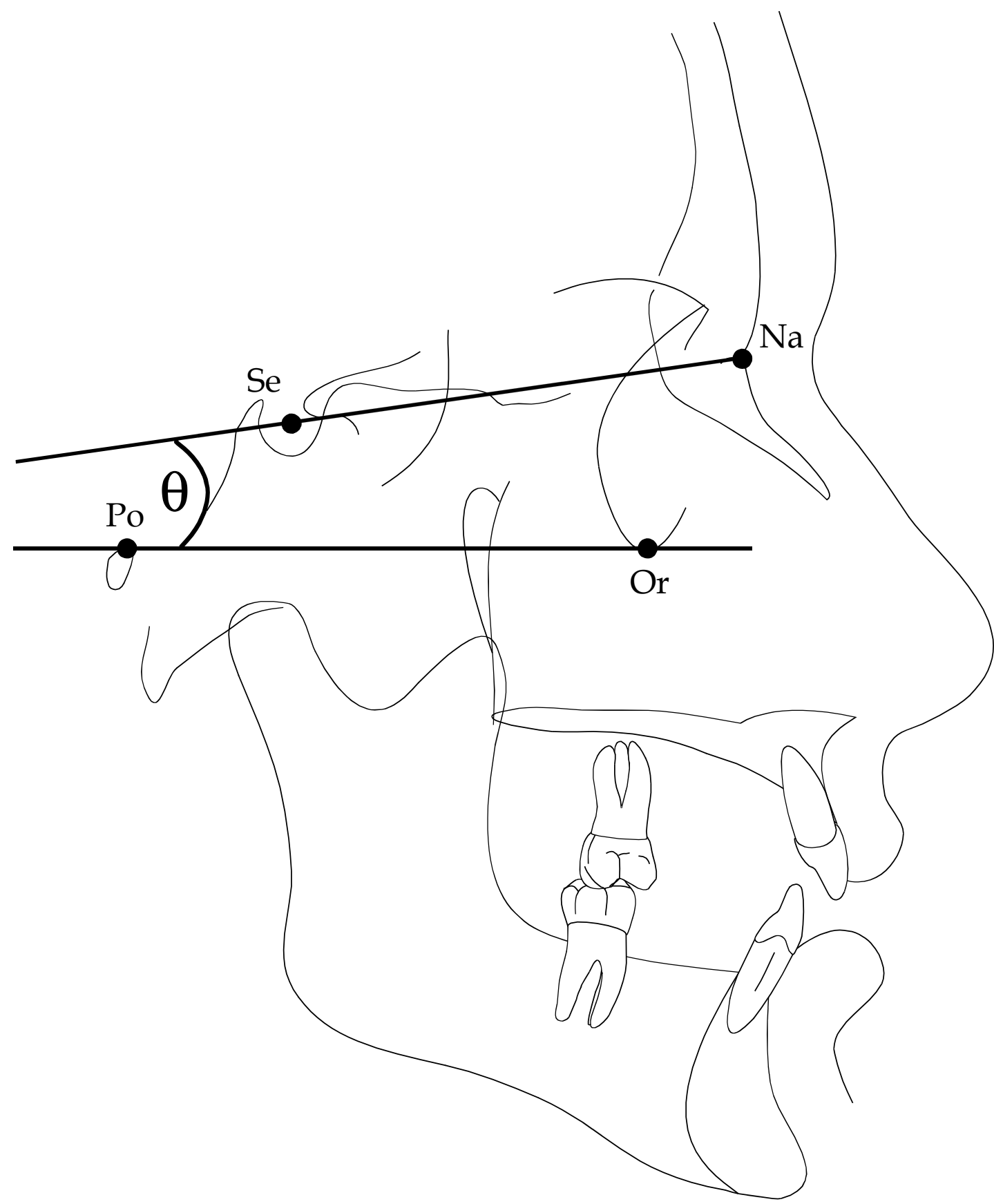

Figure 8: Schematic tracing of a lateral cephalogram showing construction of the angle formed by the intersection of Frankfort Horizontal and Se-Na line. 


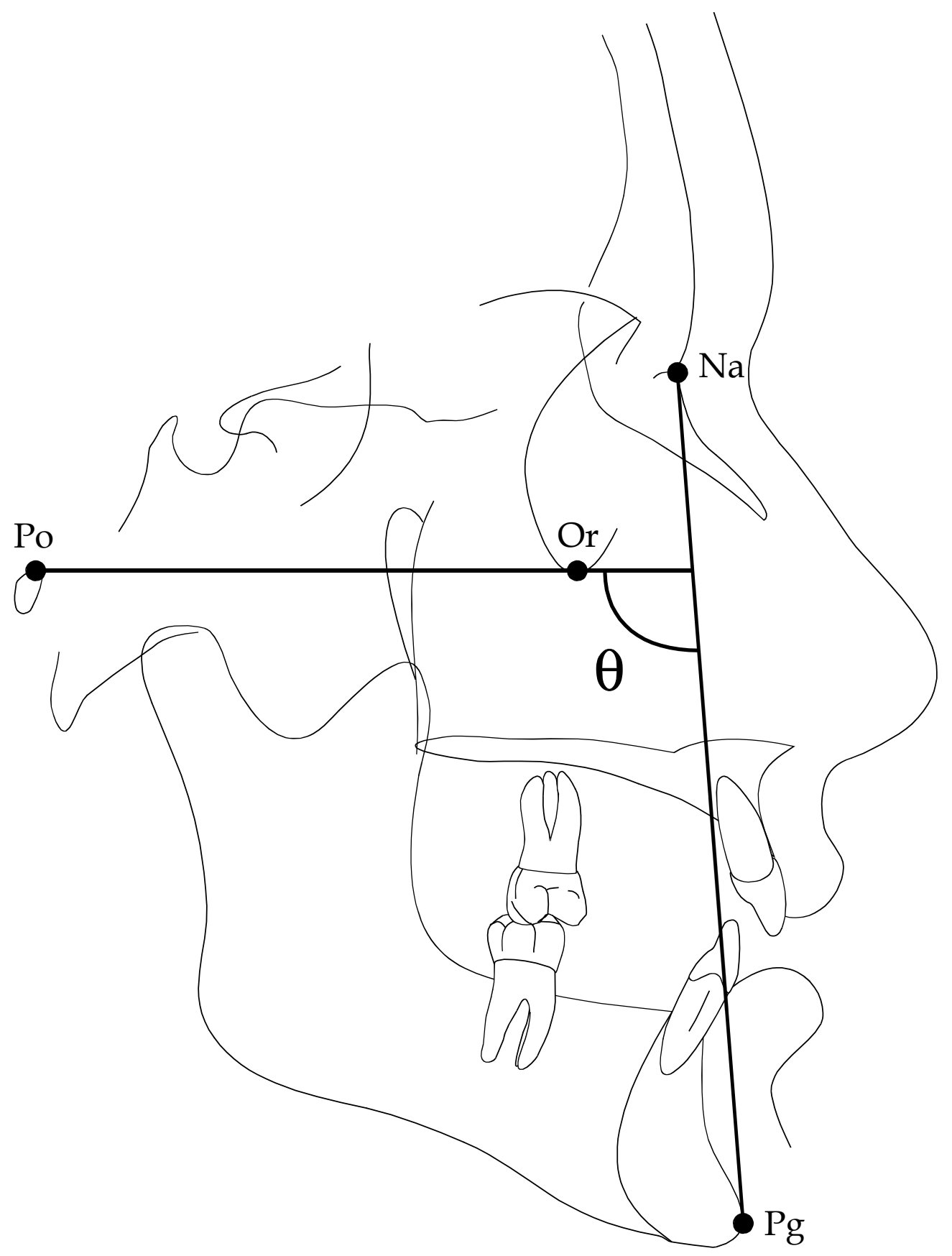

Figure 9: Schematic tracing of a lateral cephalogram showing construction of Down's Facial Angle. This is the angle formed by the posterior-inferior intersection of Frankfort Horizontal and Nasion-Pogonion lines. 


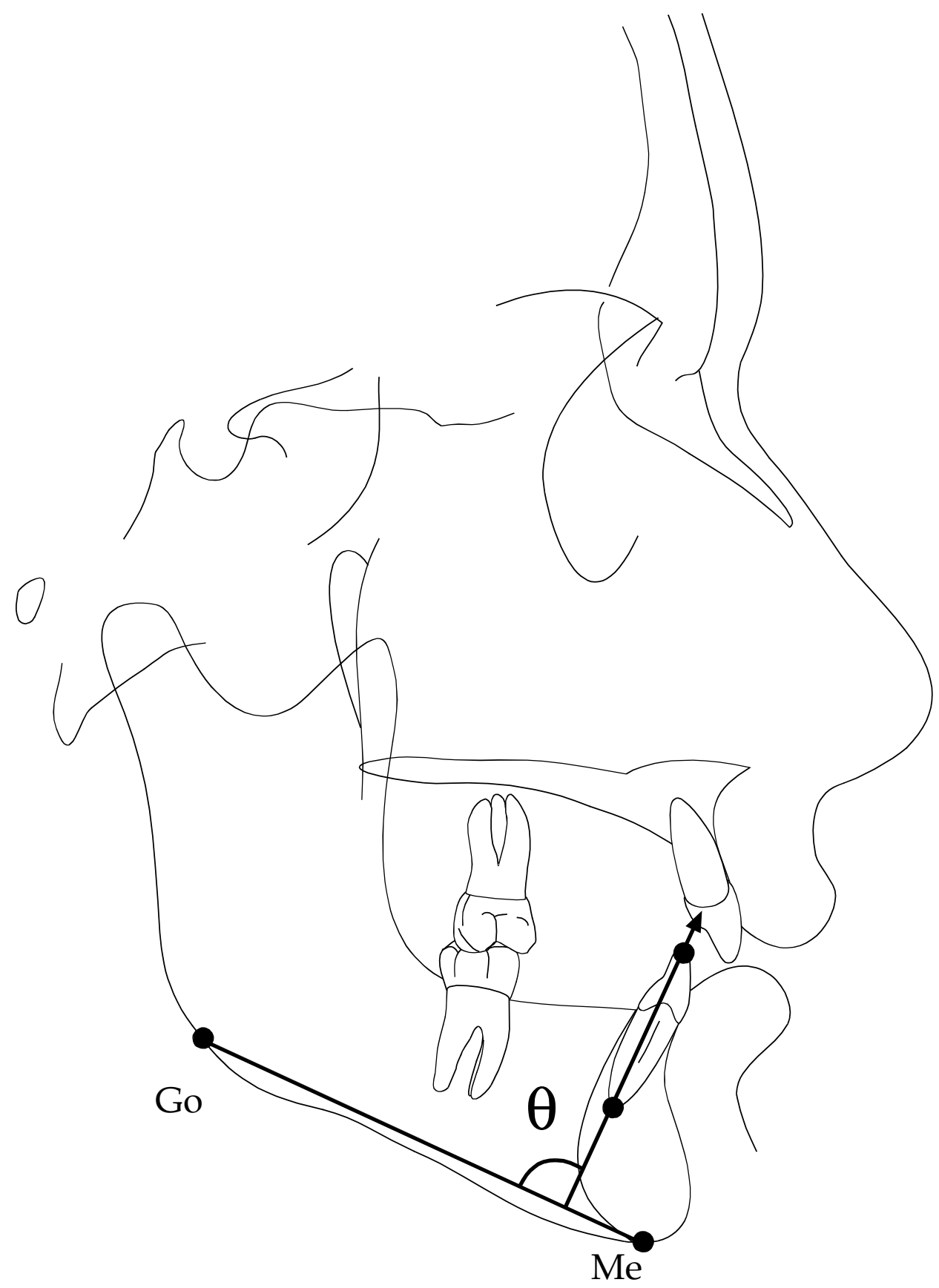

Figure 10: Schematic tracing of a lateral cephalogram showing construction of the angle of the mandibular incisor and the mandibular plane (IMPA). 


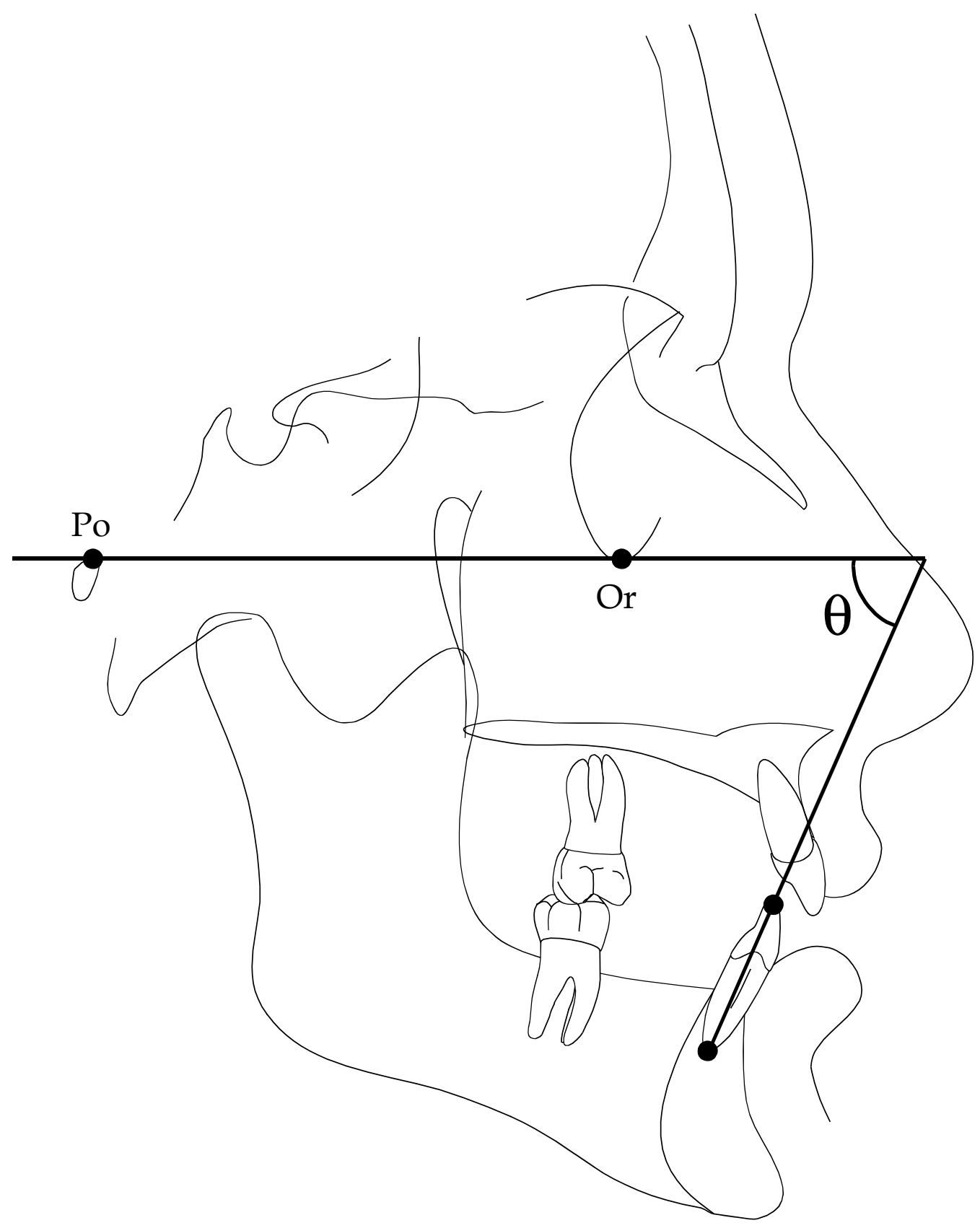

Figure 11: Schematic tracing of a lateral cephalogram showing construction of FMIA measurement. This is formed by the intersection of Frankfort Horizontal and the long axis of the mandibular incisor (L1 apex and L1 incisal edge). 


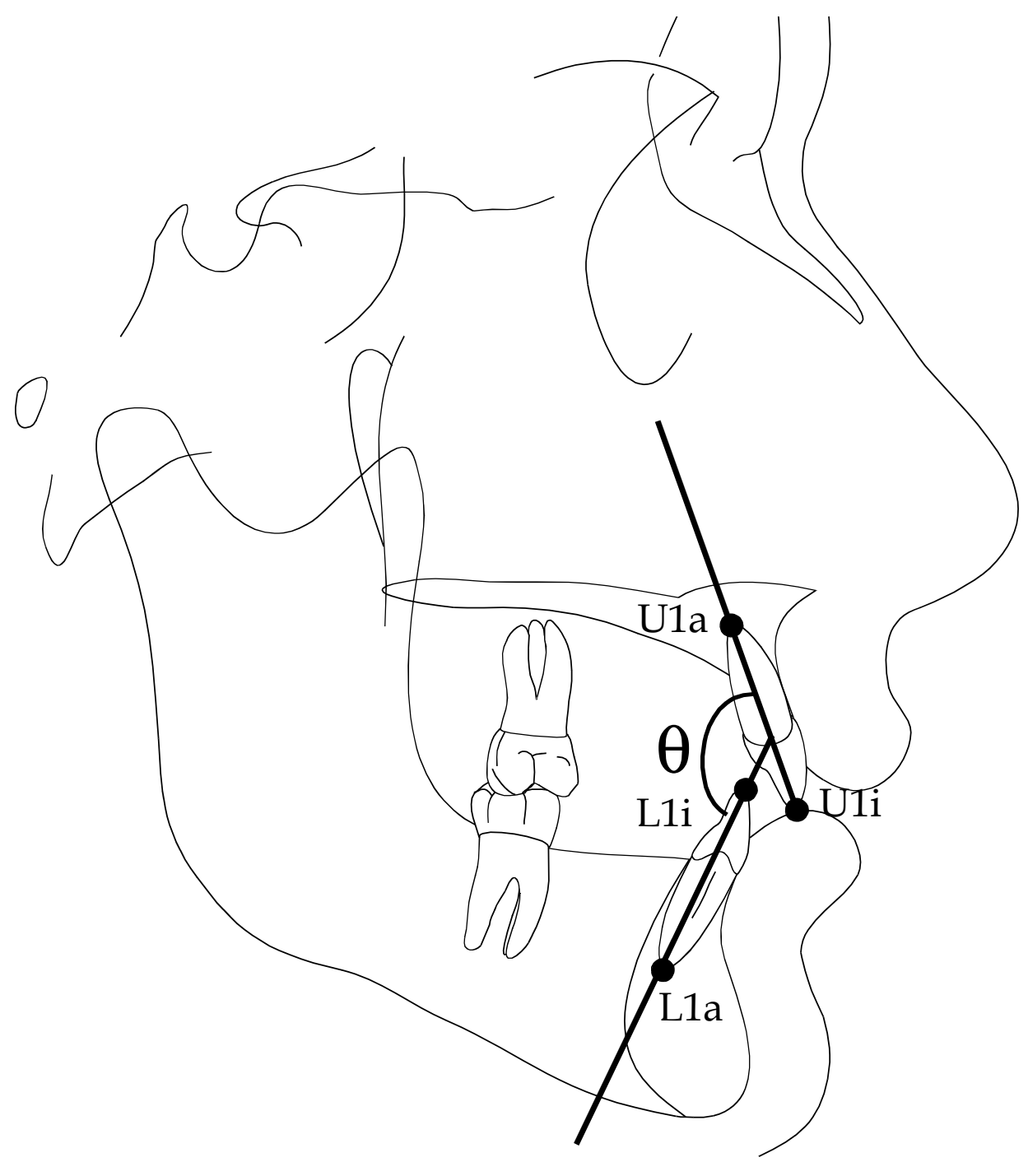

Figure 12: Schematic tracing of a lateral cephalogram showing construction of the angle between the upper incisor with the lower incisor (i.e., interincisal angle). 

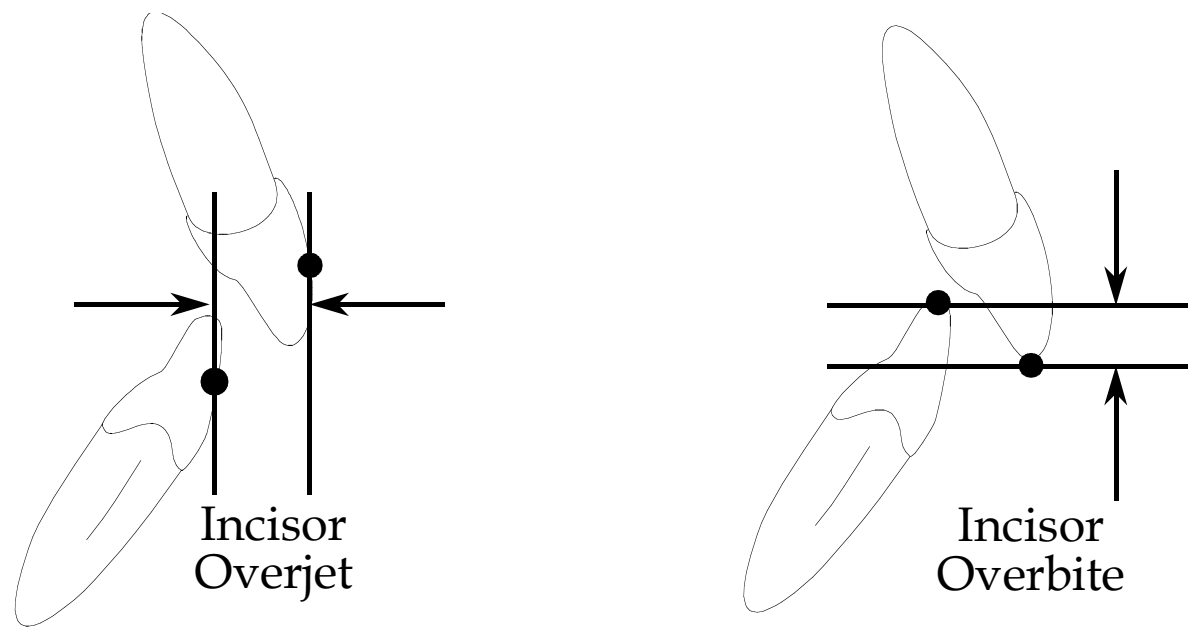

Figure 13: Schematic tracing of overbite and overjet measurements. 


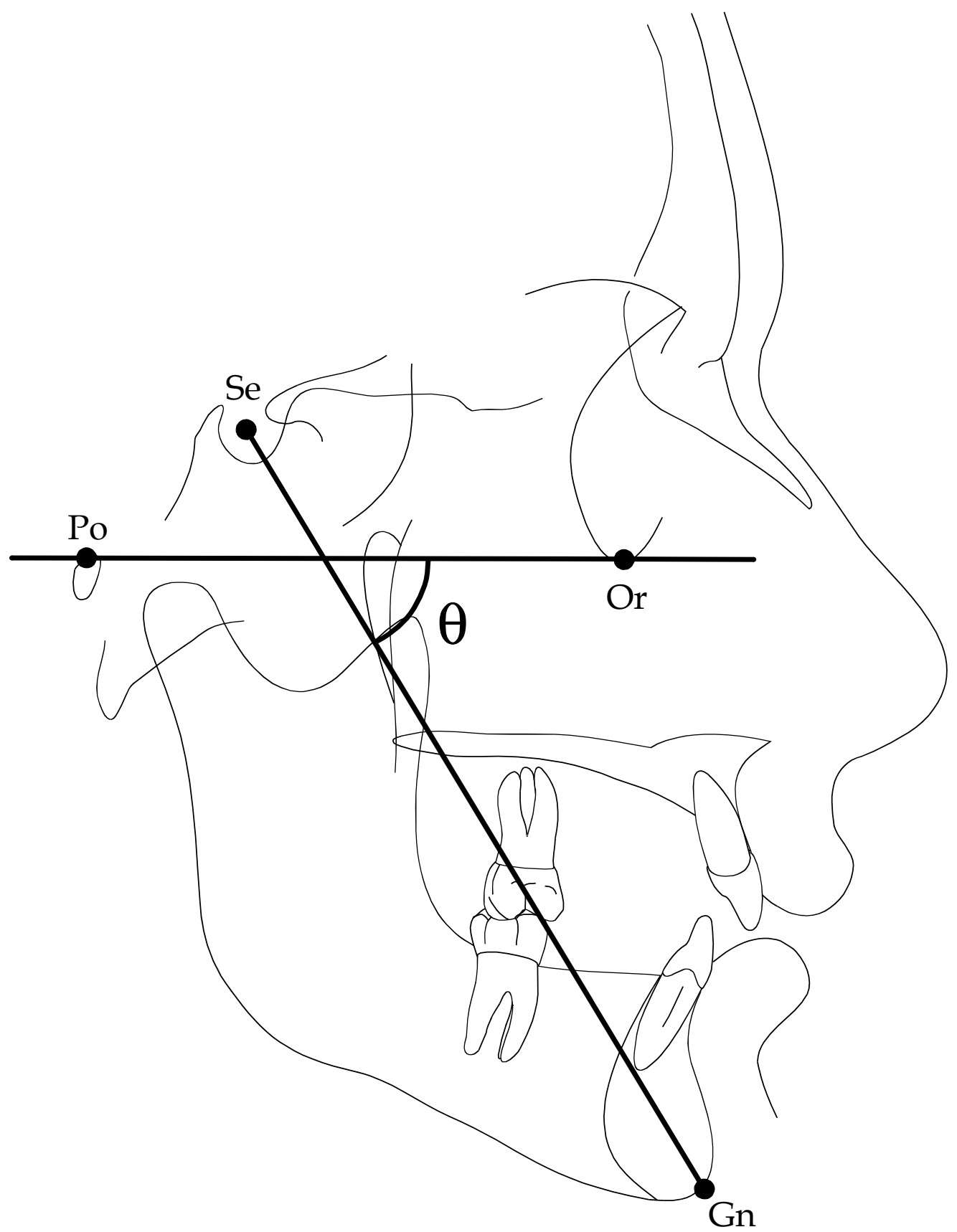

Figure 14: Schematic tracing of a lateral cephalogram showing construction of the $Y$ axis angle. This is the anterior-inferior angle at the intersection of Frankfort Horizontal and the Sella-Gnathion line. 


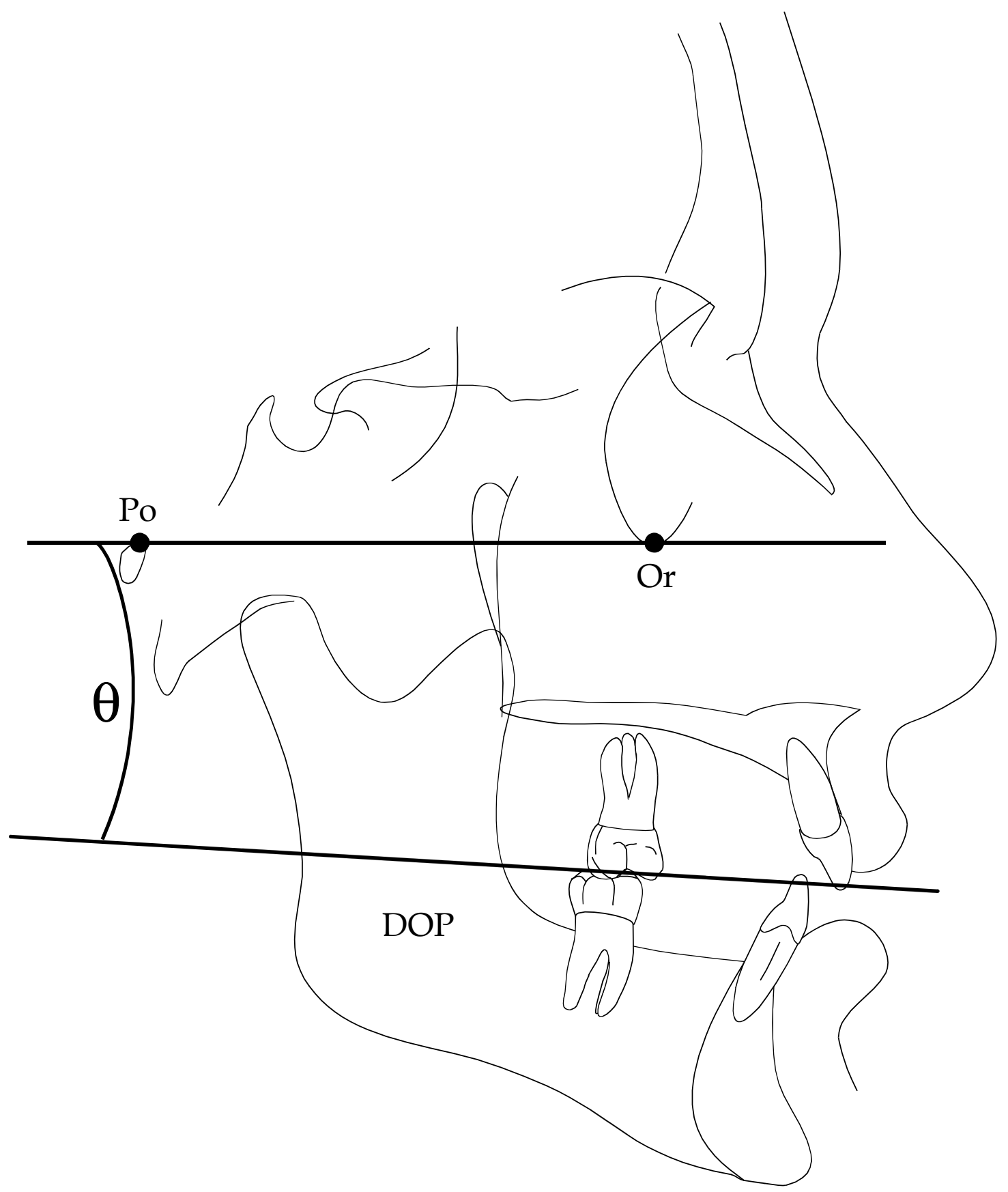

Figure 15: Schematic tracing of a lateral cephalogram showing construction of the Down's occlusal plane angle. This is the angle formed by the intersection of Frankfort Horizontal and Down's occlusal plane. 


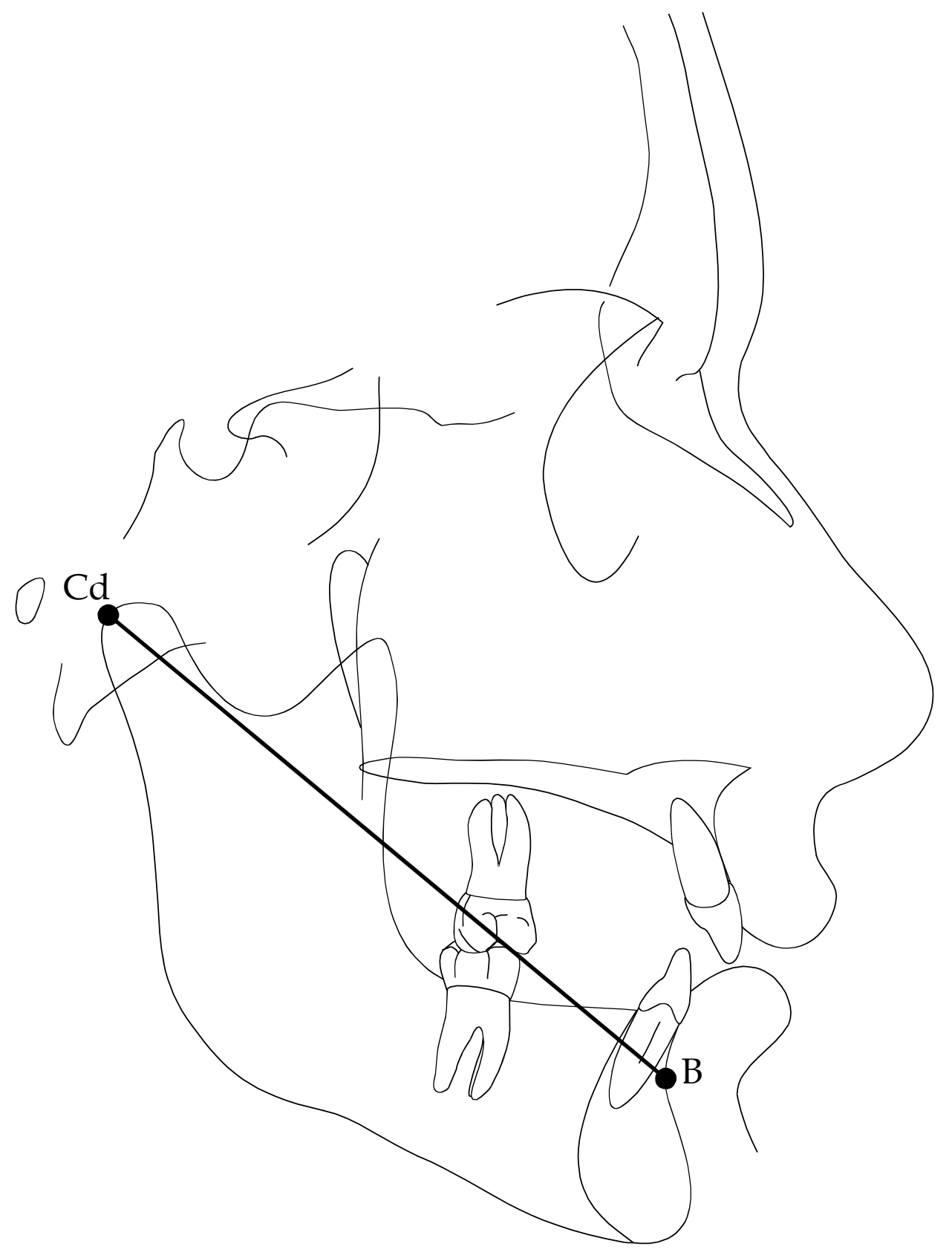

Figure 16: Schematic tracing of a lateral cephalogram showing construction of the Condylion-B Point. 


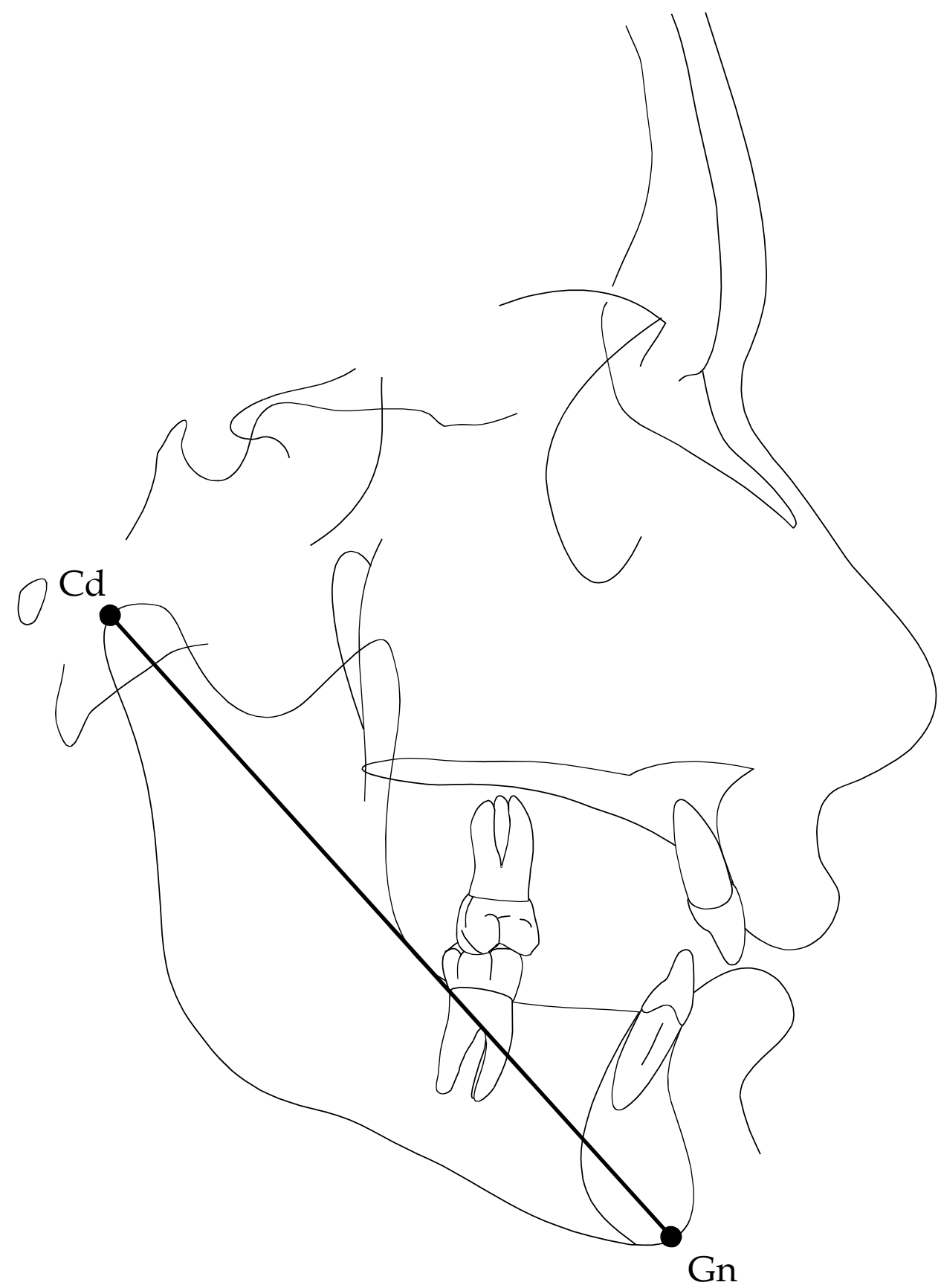

Figure 17: Schematic tracing of a lateral cephalogram showing construction of the Condylion to Gnathion. 
spreadsheet $\left(\right.$ Microsoft $\left.^{\circledR}\right)$, then transferred to the statistical package termed JMP 5.0.2 (SAS Institute Inc., Cary, NC). Exploratory data analysis (Tukey 1977) was performed, searching for outliers; those due to technical errors were corrected.

Conventional descriptive statistics (e.g., Sokal and Rohlf 1995) were calculated; these (and abbreviations) are sample size (n, taken as counts of individuals, not sides), the arithmetic mean $(\bar{x})$, the standard deviation (sd), and the standard error of the mean (sem, calculated as $\mathrm{sd} / \sqrt{ } \mathrm{n})$. The conventional alpha level of 0.05 was used throughout, and all of the tests were two-tail. No correction was made for multiple comparisons. Salient results of the analysis were graphed using Delta Graph ${ }^{\circledR} 4.0 .5$ for the PC.

The data were used to compare treatments with regard to (1) whether changes occurred during the first phase treated with a functional appliance, (2) whether changes occurred during the second phase treated with a fixed appliance, and (3) whether the amounts of change differed between the two phases for the experimental group. The differences were calculated at the start of treatment and the progress record, then between the progress record and the end of treatment. One sample t-tests (two tail) were used to evaluate changes in variables across time as a function of the sample variability. Repeated measures analysis of variance (Winer et al. 1991) was used to determine if the change from phase one to progress differed significantly from the change observed in phase two. 
Statistical descriptions for the one-phase treatment group were calculated by obtaining the descriptive statistics for the sample at the two examinations, and then separately using pairing-design t-tests to assess whether the treatment change differed significantly from the null hypothesis of no change (two tail). To assess how comparable the skeletodental variables were at the end of treatment we calculated the difference in the arithmetric means of the one- and two-phase groups. In addition, a paired t-test was calculated to determine if the two groups differ statistically. 


\section{CHAPTER IV}

\section{RESULTS}

\section{$\underline{\text { Time in Treatment }}$}

This sample of two-phase treatments began treatment at an average of 8.8 years $(\mathrm{sd}=0.15$ years), and treatment lasted an average of 5.1 years $(\mathrm{sd}=$ 1.52 years), so the children were an average of 13.9 years of age ( $\mathrm{sd}=1.45$ years) at completion (Table 1). The first "functional" phase was, on average, 2.5 years in duration ( $\mathrm{sd}=1.00$ years), so the children averaged 11.2 years of age at the start of fixed appliances, which had an average duration of 2.6 years $(\mathrm{sd}=1.10$ years). Mean age at the end of treatment was 13.8 years ( $\mathrm{sd}=1.35$ years).

The one-phase group began treatment at an average age of 13.1 years (sd $=1.32$ years $)$, with a mean time in treatment of 3.1 years $(\mathrm{sd}=2.58$ years $)$. The average age at completion was 16.2 years ( $\mathrm{sd}=2.93$ years).

Overall time in treatment for the two-phase group was, then, 5.2 years $(\mathrm{sd}=1.32$ years) compared to the 3.1 in the single-phase group. Fixed appliances were used for 2.6 years ( $\mathrm{sd}=1.10$ years) in the two-phase group, which is 0.5 years (6 months) shorter than in the one-phase treatment. 
Table 1. Comparison of chronological age and time in treatment between the two treatment groups.

\begin{tabular}{lccccr}
\hline Variable & $\begin{array}{c}\text { One } \\
\text { Phase }\end{array}$ & $\begin{array}{c}\text { Two } \\
\text { Phase }\end{array}$ & $\begin{array}{c}\text { Mean } \\
\text { Difference }\end{array}$ & t test & P value \\
\hline Age at Start & 13.09 & 8.82 & 4.28 & 16.10 & $<0.0001$ \\
Time in Treatment & 3.15 & 5.08 & -1.93 & -3.73 & 0.0008 \\
\hline
\end{tabular}




\section{One-Phase Treatment}

This sample of 32 subjects was treated with conventional edgewise mechanics, and the descriptive statistics based on the pretreatment and posttreatment cephalograms are listed in Table 2. The statistical descriptions were calculated by obtaining the descriptive statistics for the sample at the two examinations, and then pairing-design t-tests were used to assess whether the treatment change differed significantly from the null hypothesis of no change (two tail). These observed changes are some composite of treatment plus growth.

There were statistically significant improvements of both measures of lip profiles ( $\mathrm{Z}$ angle, $\mathrm{E}$ plane). The $\mathrm{Z}$ angle steepened an average of $4.1^{\circ}$, meaning that the lower lip became more prominent, which is desirable in these Class II, retrognathic subjects. Comparably, the lower lip to E plane moved from behind this plane at the start of treatment to in front of the plane at the end of treatment, a mean change of $1.5 \mathrm{~mm}(\mathrm{P}=0.0026)$.

There were several significant skeletal changes. Usefully, though, the $Y$ axis did not change (Ricketts 1961). There are four cephalometric measures that measure changes in relationships between the two jaws, namely NAP, ANB, AOBO, and the Facial Angle (Table 2). Each of these exhibited a highly significant change in a favorable direction. NAP decreased $4.2^{\circ}(\mathrm{P}<0.0001)$. ANB decreased an average of $1.5^{\circ}(\mathrm{P}<0.0001)$. AOBO diminished $3.1 \mathrm{~mm}(\mathrm{P}<$ 


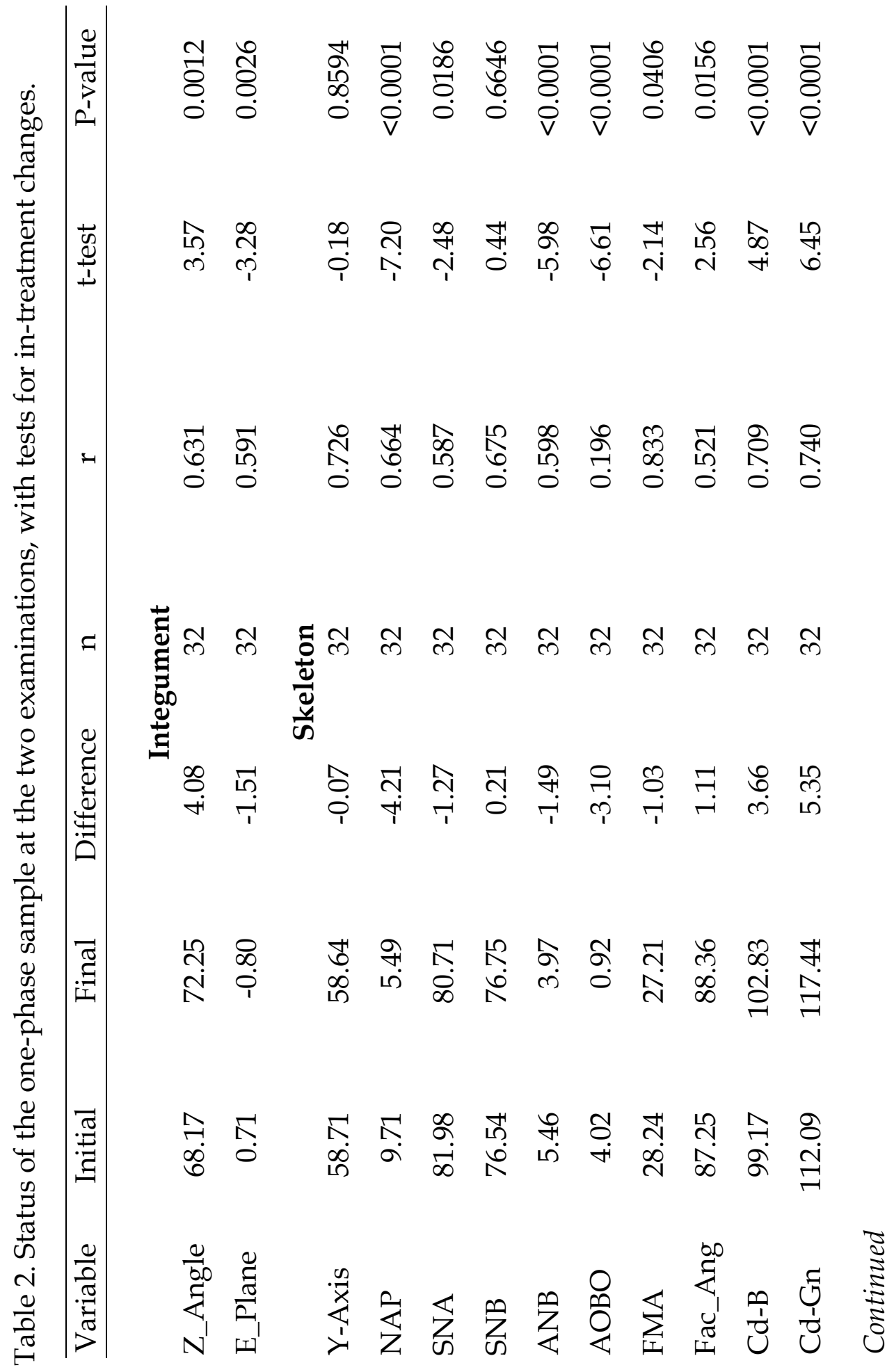




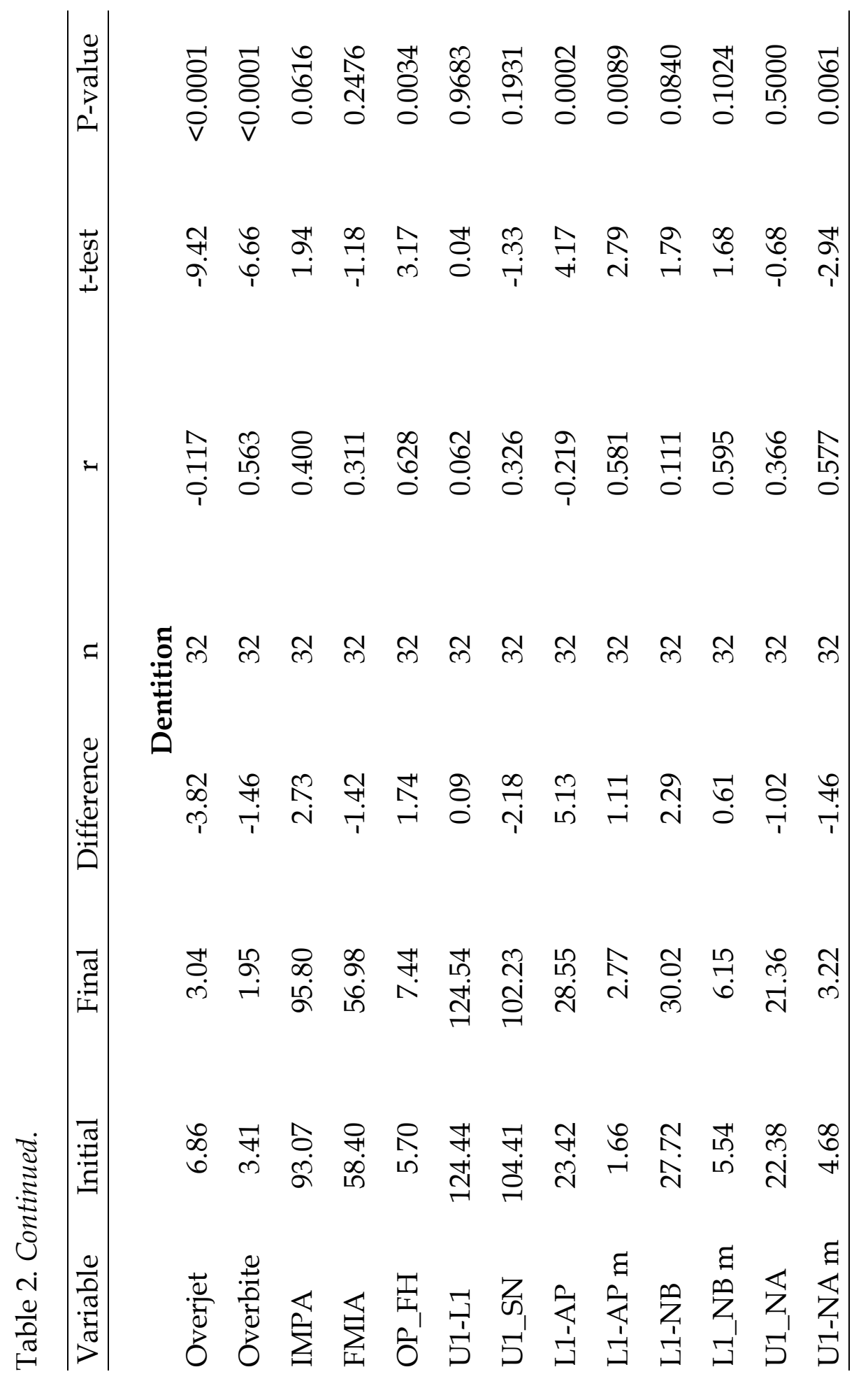


0.0001). Facial Angle increased $1.1^{\circ}(\mathrm{P}=0.02)$. These changes show that the facial profile becomes less retrognathic (as a result of treatment plus growth) between the ages of about 13 and 16 years of age.

It appears that much of the sagittal improvement in jaw relationships was achieved by mandibular growth. For example, AOBO improved an average of $3.1 \mathrm{~mm}$, which is comparable to the $3.7 \mathrm{~mm}$ advancement recorded for Condylion-B Point. The other measure of mandibular growth used here, Condylion-to-Gnathion, showed a mean improvement of $5.3 \mathrm{~mm}(\mathrm{P}<0.0001)$. Looking at the 13 dental variables measured here, six changed significantly over the course of treatment (Table 2). Overjet diminished $3.8 \mathrm{~mm}$ on the average $(\mathrm{P}<0.0001)$, and overbite decreased from $3.4 \mathrm{~mm}$ to $2.0 \mathrm{~mm}$, which also was highly significant statistically. The occlusal plane (based on Downs' occlusal plane) steepened $1.7^{\circ}(\mathrm{P}=0.003)$. The mandibular incisor uprighted $5.1^{\circ}$ (L1 to AP), which was highly significant $(\mathrm{P}=0.0002)$. Comparably, the L1 to AP distance diminished an average of $1.1 \mathrm{~mm}(\mathrm{P}=0.009)$. The other significant change was of the upper incisor to the Nasion-A Point line (U1-NA) measured millimetrically. This distance diminished an average of $1.5 \mathrm{~mm}(\mathrm{P}=$ $0.006)$. 


\section{$\underline{\text { Start of Treatment }}$}

Our research design was to match an orthodontic case, by sex and cephalometric values, to each of the 32 cases treated with the Fränkel appliance. Matching criteria focused on the bony facial characteristics, notably ANB, NAP, and the $\mathrm{Y}$ axis. Table 3 lists the descriptive statistics for the one- and two phase samples, along with pairing design t-tests that evaluated the differences between groups.

Our ability to find reasonable matches for the two-phase group seems to have been good since just one of the eight bony variables differed significantly, and this did not involve the features that we focused on. Indeed, all of the mean differences between groups were less than one degree (or one millimeter) except for SNA and AOBO. The angle SNA was non-significantly larger in the one-phase group $\left(82.0^{\circ}\right)$ versus $80.8^{\circ}$ in the two-phase group $(\mathrm{P}=0.14)$. The one significant skeletal difference was $\mathrm{AOBO}$, which was larger in the onephase group $(\bar{x}=4.0 \mathrm{~mm})$ than the two-phase sample $(\bar{x}=0.9 \mathrm{~mm})$ at $P=$ 0.002 .

To reemphasize, the two groups were adequately matched for $\mathrm{Y}$ axis, NAP, SNA, SNB, ANB, FMA, and the Facial Angle at the start of treatment. Examination of Table 3 also shows that the facial profile (assessed by the $\mathrm{Z}$ Angle and the E plane) were comparable. 


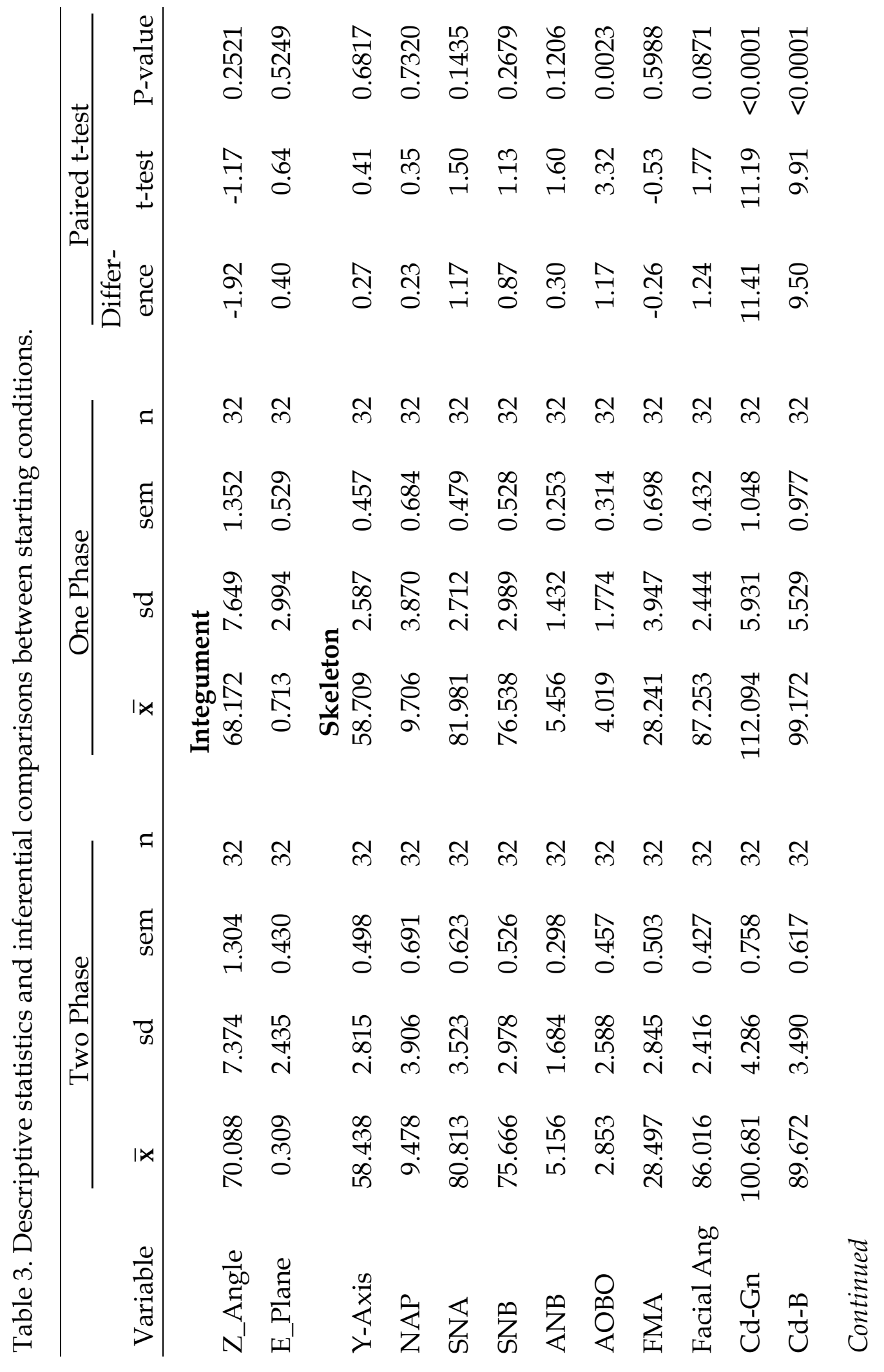




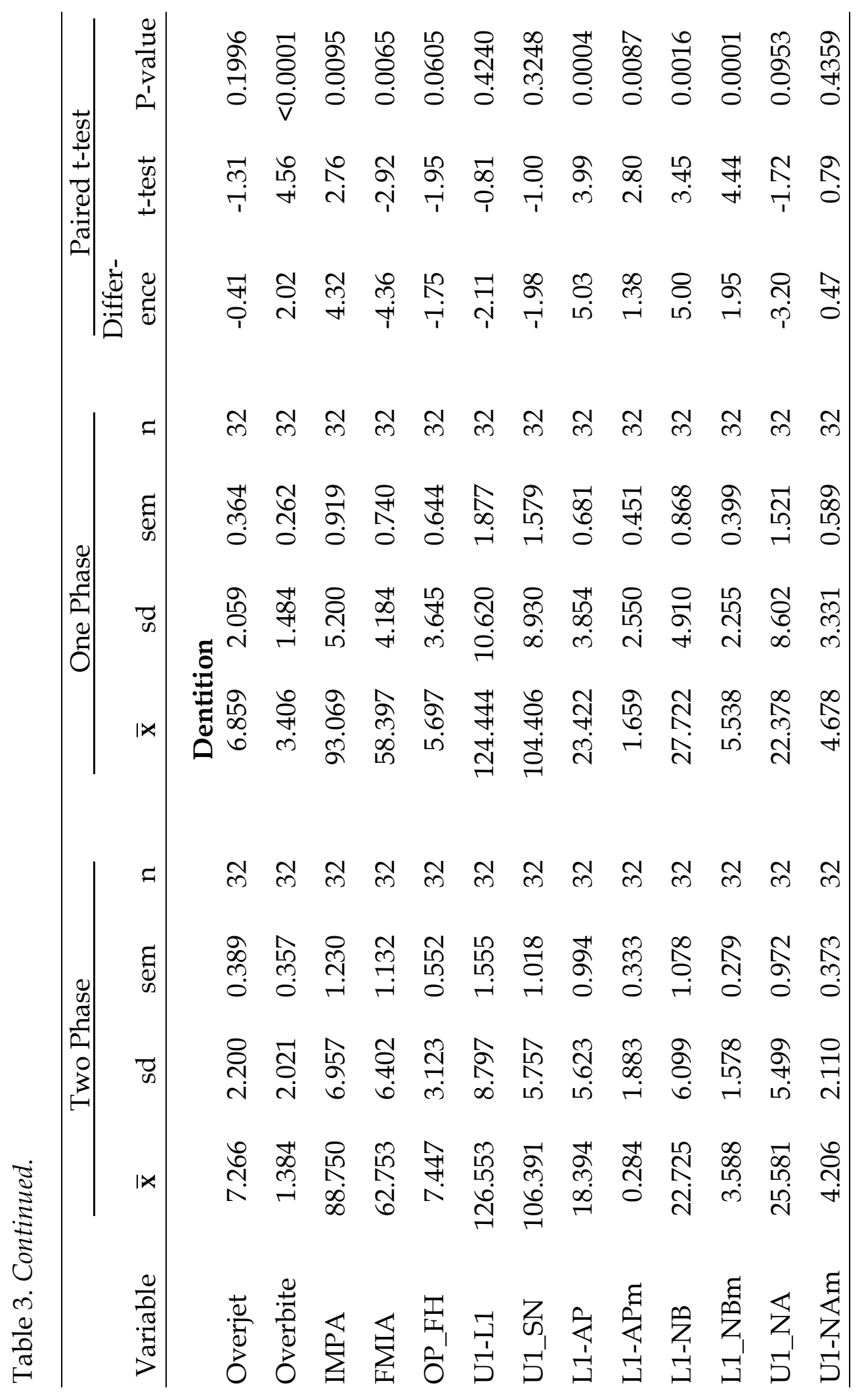


No attention was paid to the dental relationships during the matching process, other than the obvious need that all cases had a Class II, division 1 malocclusion, so it is not surprising that several of the 11 dental variables (6 of 11) differed between the two groups (Table 3).

IMPA showed that the lower incisor was proclined significantly more in the one-phase group $\left(93.1^{\circ}\right.$ versus $\left.88.8^{\circ}\right)$, and, comparably, FMIA was smaller in the one-phase sample $\left(58.4^{\circ}\right.$ versus $\left.62.8^{\circ}\right)$. Of note, though, interincisal angle was very similar in the two groups at about $125^{\circ}(\mathrm{P}=0.42)$.

There are four other significant dental differences listed in Table 3, but they all reflect the same issue, namely that the lower central incisor was proclined less and positioned farther back in the one-phase group. This is the inference from comparison of L1-AP, L1-A millimetric, L1-NB, and L1-NB millimetric.

In overview, then, matching of individuals in the one-and two-phase samples based on key cephalometric skeletal characteristics was successful (Table 3), and all cases were Class II, division 1. Rather little emphasis was placed on the dental relationships because both groups underwent comparable sessions of full-banded treatment before the final assessment. One possibility is that the dental malocclusions are somewhat worse in the one-phase group because they are about five years older. The merit of creating matched samples is that it is more efficient than a group comparison test and is more likely to 
disclose a significant difference if one exists (e.g., Woolf 1968). One important consideration is the skeletal difference between the two groups in mandibular size. Table 3 shows that there are large differences for the Condylion-Gnathion and Condylion-B Point distances. The rationale is that there had to be adjustment for age. To correlate mandibular length at different ages one would need a mean annualized change in mandibular length in Class II subjects receiving no treatment (i.e., observation). Mills and McCulloch (2000) conducted a cephalometric study that investigated treatment changes over time in Class II subjects that were either treated with the twin block appliance or observed (i.e., no treatment). The mean age of the control group $(n=28)$ was 9 years 1 month at the start of the observation period and 12 years 11 months approximately 4 years later. The control group experienced a mean increase in mandibular unit length (Condylion-Gnathion) of $2.3 \mathrm{~mm}$ during the first 13 months of observation. Over the next 36 months of observation the control group experienced an average increase in mandibular unit length of $6.7 \mathrm{~mm}$ or an annualized rate of change of $2.4 \mathrm{~mm}$ per year. Toth and McNamara (1999) in a similar study observed a mean change in mandibular unit length (Condylion-Gnathion) of $2.7 \mathrm{~mm}$ over a 16 month period in an untreated Class II control (mean age of 10 years 5 months). Janson and coworkers (2003) observed an untreated Class II division 1 control group over a 28 month period mean age of 8 years and 5 months to 11 years 6 months. A mean increase in 
mandibular length (Condylion-Gnathion) of $5.0 \mathrm{~mm}$ was observed. Almeida et al. (2002) also observed an untreated control group of Class II division 1 subjects over a 13 month period and noted a mean increase in mandibular length (Condylion-Gnathion) of $3.0 \mathrm{~mm}$. Hamilton and coworkers (1987) also examined untreated Class II division 1 subjects over a 2 year period and observed a mean annualized increase in mandibular length (Condylion-B Point) of $2.0 \mathrm{~mm}$ per year.

\section{End of Treatment}

One important consideration in this study is how comparable the skeletodental variables are at the end of treatment. Results are listed in Table 4 . The important statistics in this table are the arithmetic means of the one-and two-phase groups, the difference between the means, and a paired t-test of whether the two groups differ statistically. On inspection, rather few of the 21 variables differed significantly.

Neither integumental variable ( $\mathrm{Z}$ angle, E plane) differed at an alpha of 0.05 , but both differ at $0.10>\mathrm{P}>0.05$, which is suggestive. Both variables are at more desirable values in the one-phase group, showing that the lower lip is less retrusive. Again, though, neither variable truly differs statistically.

Two of the eight skeletal variables differs statistically, namely ANB and FMA. ANB is greater in the one-phase sample. That is, ANB is significantly 
Table 4. Comparison of skeletodental means between the two treatment groups at the end of treatment.

\begin{tabular}{|c|c|c|c|c|c|c|c|}
\hline Variable & $\begin{array}{l}\text { One } \\
\text { Phase }\end{array}$ & $\begin{array}{l}\text { Two } \\
\text { Phase }\end{array}$ & $\begin{array}{c}\text { Mean } \\
\text { Difference }\end{array}$ & sem & $\mathrm{n}$ & t-test & P-value \\
\hline \multicolumn{8}{|c|}{ Integument } \\
\hline Z Angle & 72.25 & 75.65 & -3.40 & 1.80 & 32 & -1.89 & 0.0679 \\
\hline E_Plane & -0.80 & -1.86 & 1.06 & 0.62 & 32 & 1.72 & 0.0957 \\
\hline \multicolumn{8}{|c|}{ Skeleton } \\
\hline Y-Axis & 58.64 & 59.31 & -0.66 & 0.68 & 32 & -0.97 & 0.3388 \\
\hline NAP & 5.49 & 3.12 & 2.37 & 1.41 & 32 & 1.68 & 0.1026 \\
\hline SNA & 80.71 & 79.62 & 1.09 & 1.00 & 32 & 1.09 & 0.2860 \\
\hline SNB & 76.75 & 77.00 & -0.25 & 0.96 & 32 & -0.26 & 0.7928 \\
\hline ANB & 3.97 & 2.63 & 1.34 & 0.58 & 32 & 2.31 & 0.0279 \\
\hline AOBO & 0.92 & 0.18 & 0.74 & 0.66 & 32 & 1.12 & 0.2697 \\
\hline FMA & 27.21 & 28.98 & -1.77 & 0.82 & 32 & -2.16 & 0.0388 \\
\hline Facial Ang & 88.36 & 88.08 & 0.28 & 0.70 & 32 & 0.41 & 0.6857 \\
\hline Cd-Gn & 117.44 & 115.28 & 2.17 & 1.22 & 32 & 1.78 & 0.0846 \\
\hline Cd-B & 102.83 & 101.26 & 1.58 & 1.02 & 32 & 1.55 & 0.1318 \\
\hline \multicolumn{8}{|c|}{ Dentition } \\
\hline Overjet & 3.04 & 3.22 & -0.17 & 0.18 & 32 & -0.93 & 0.3596 \\
\hline Overbite & 1.95 & 2.22 & -0.27 & 0.24 & 32 & -1.13 & 0.2673 \\
\hline IMPA & 95.80 & 90.00 & 5.80 & 1.54 & 32 & 3.76 & 0.0007 \\
\hline FMIA & 56.98 & 61.04 & -4.06 & 1.37 & 32 & -2.96 & 0.0058 \\
\hline OP-FH & 7.44 & 6.39 & 1.05 & 0.91 & 32 & 1.15 & 0.2576 \\
\hline U1-L1 & 124.54 & 127.96 & -3.42 & 1.94 & 32 & -1.76 & 0.0886 \\
\hline U1_SN & 102.23 & 103.08 & -0.85 & 1.62 & 32 & -0.52 & 0.6046 \\
\hline L1-AP & 28.55 & 25.48 & 3.07 & 1.23 & 32 & 2.50 & 0.0179 \\
\hline L1-AP m & 2.77 & 3.02 & -0.25 & 0.47 & 32 & -0.54 & 0.5930 \\
\hline L1-NB & 30.02 & 25.96 & 4.06 & 1.19 & 32 & 3.40 & 0.0018 \\
\hline L1-NB m & 6.15 & 5.56 & 0.59 & 0.46 & 32 & 1.27 & 0.2137 \\
\hline U1_NA & 21.36 & 23.46 & -2.10 & 1.56 & 32 & -1.35 & 0.1882 \\
\hline U1-NA m & 3.22 & 4.90 & -1.68 & 0.84 & 32 & -1.99 & 0.0555 \\
\hline
\end{tabular}


smaller in the two-phase sample $\left(\bar{x}=2.6^{\circ}\right)$ than the one-phase sample $(\bar{x}=$ $4.0)$, and this difference of $1.3^{\circ}$ is significant statistically $(\mathrm{P}=0.03)$.

The mandibular plane angle also differed at the end of treatment. The two-phase sample had a final FMA averaging $29.0^{\circ}$, which is $1.8^{\circ}$ steeper than the one-phase sample $(\mathrm{P}=0.0388)$.

Four of the eleven dental variables differed at the end of treatment. IMPA finished at $90^{\circ}$ in the two-phase group, which is $5.8^{\circ}$ less than in the onephase sample $\left(\bar{x}=95.8^{\circ}\right)$, which is a highly significant difference. FMIA ended at a mean of $61.0^{\circ}$ in the two-phase group, which is $4.1^{\circ}$ greater than in the onephase sample $(P=0.0058)$. Two measures of the mandibular central incisors also differed. The angulation of L1 to the AP line was $25.5^{\circ}$ in the two-phase group, which on average is $3.1^{\circ}$ smaller than in the one-phase group $(\mathrm{P}=$ 0.0179). Comparably, the angle between L1 and the NB line was $26.0^{\circ}$ in the two-phase group and $4.1^{\circ}$ larger $\left(\overline{\mathrm{x}}=30.0^{\circ}\right)$ in the one-phase group $(\mathrm{P}=$ 0.0018). Overall, then, the incisors in both treatment groups proclined during treatment. It should be noted that the two-phase group began treatment with the lower incisors more retroclined with respect to both FMIA and IMPA.

It seems that the key feature in these comparisons (Table 4) is whether the two-phase group exhibits a significantly better (more harmonious, orthognathic) skeletal profile. There are three important variables that measure the maxillomandibular relationships, namely NAP, ANB, and AOBO. Of these, 
mean NAP was smaller in the two-phase group ( $3.1 \mathrm{~mm}$ versus $5.5 \mathrm{~mm}$ ), but the difference is nonsignificant $(\mathrm{P}=0.10)$. The difference in means is $1.3^{\circ}(\mathrm{P}=$ 0.03), indicating that the jaw relationships are slightly better in the two-phase sample at $2.6^{\circ}$ (versus $4.0^{\circ}$ in the one-phase sample). The Wits appraisal (AOBO) also is slightly better in the two-phase group $(0.2 \mathrm{~mm}$ versus $0.9 \mathrm{~mm})$, but the difference is nonsignificant $(\mathrm{P}=0.27)$.

\section{$\underline{\text { Patterns of Skeletodental Changes }}$}

We had the good fortune that the specialist who treated the functional cases was careful to take a cephalogram at the end of the first phase, so the major changes in the orthodontic variables could be evaluated. The descriptive statistics (mean, standard deviation, and standard error of the mean) are listed in Table 5. With these data, treatment could be compared as to (1) whether changes occurred during the first phase treated with a functional appliance, (2) whether changes occurred during the second phase treated with a fixed appliance, and (3) whether the amounts of change differed between these two phases.

These issues were addressed statistically by first calculating the differences between the start of treatment and the progress record (labeled "Phase One"), then between the progress record and the cephalogram taken at the end of treatment (labeled "Phase Two"). These descriptive statistics are 


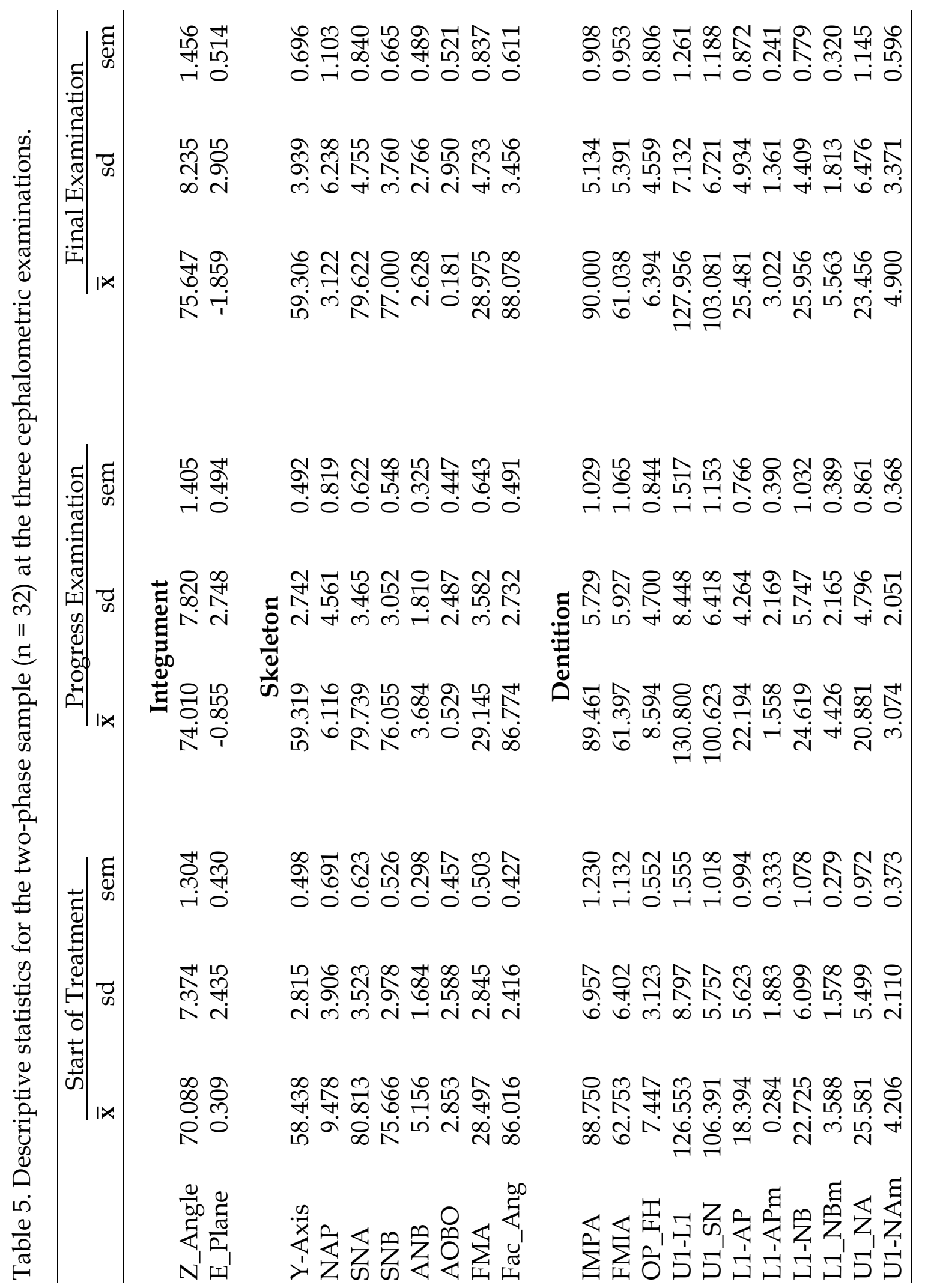


presented in Table 6. One-sample t-tests were calculated for all of these mean changes. These t-tests (two tail) evaluated whether there was a systematic change in the variable across time as a function of the sample variability. Finally, repeated measures analysis of variance was used to test whether the change during phase one differed significantly from that observed during phase two. It is worth reemphasizing that all of these steps reserve the individual's changes during treatment; the statistical comparisons do not depend on group comparison tests.

\section{Phase One}

Most of the variables tested (16/21) exhibit significant changes during the first phase of treatment. Fewer (8/21) variables changed significantly during the second phase. The changes during the two phases differ, largely because the treatment goals of each phase are different.

Both measures of lip posture improved during phase one as the lower lip moved forward into a more orthognathic relationship (Table 6). As with all of these changes, there is no way from these data alone to assess whether they are attributable to treatment or growth.

Six of the eight skeletal variables changed significantly during phase one (Table 6). The Y-axis increased because the mandible autorotated down-andback. This clockwise rotation is seen in several of the variables. Y axis was at a 


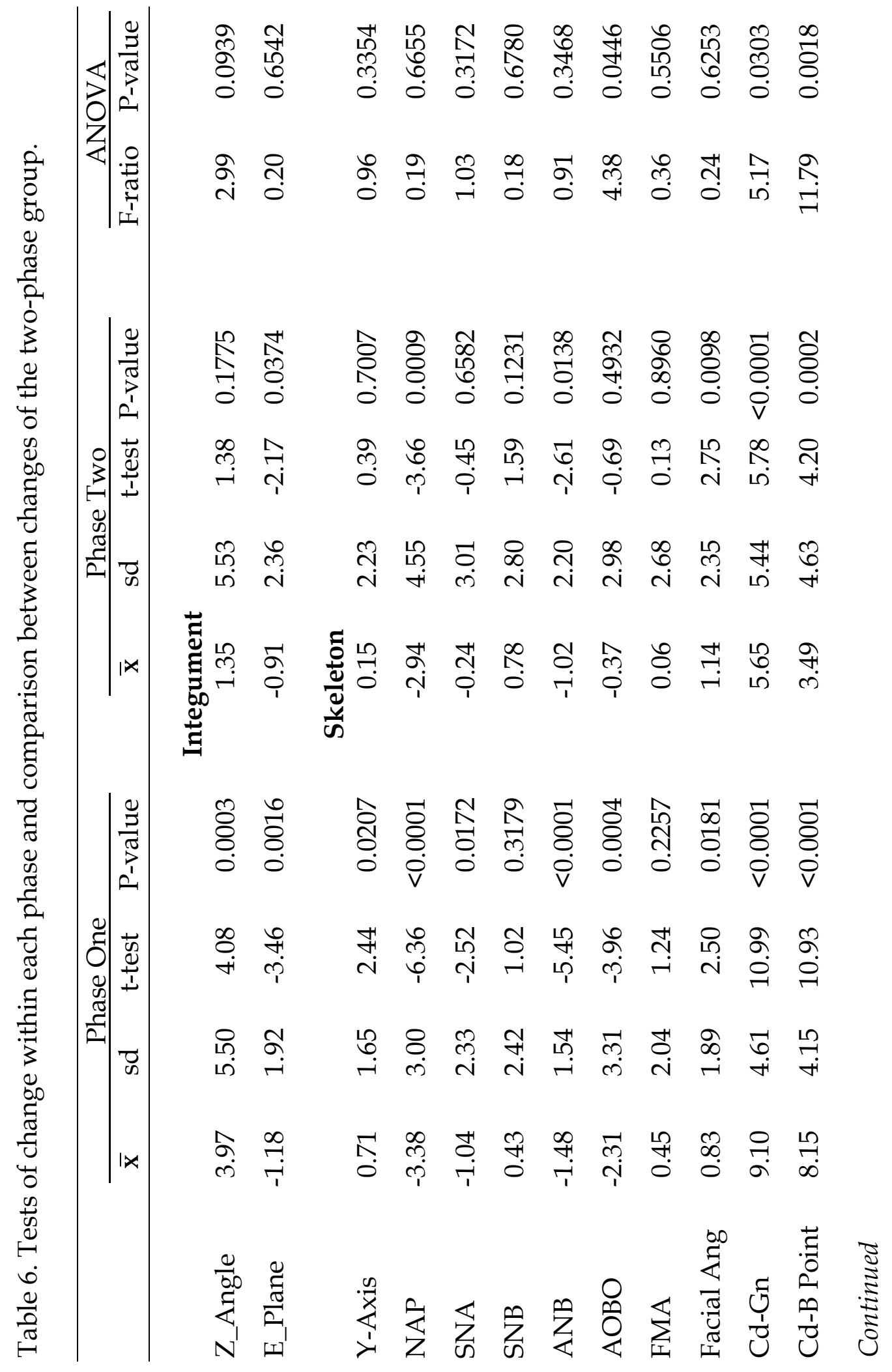




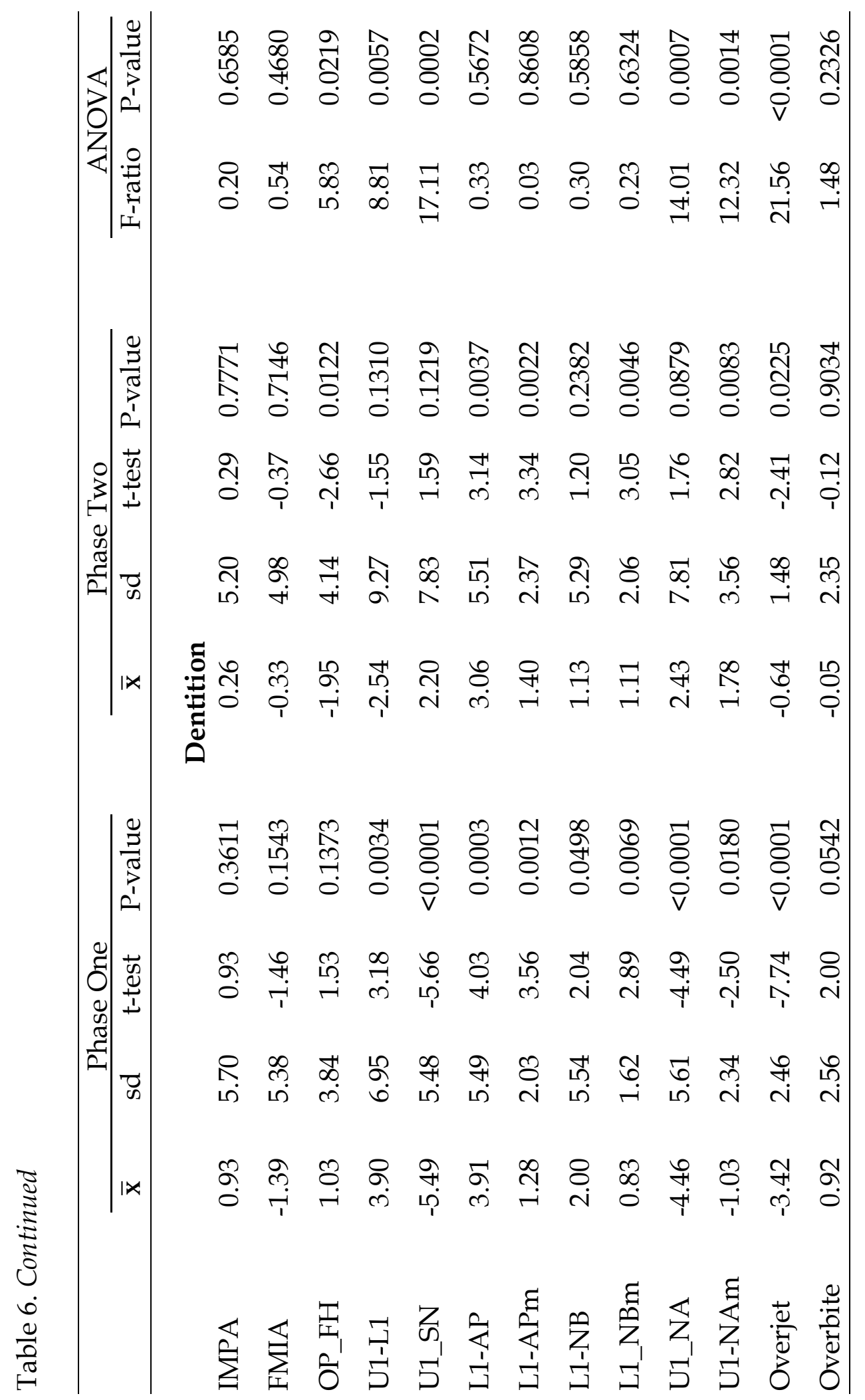


mean of $58.4^{\circ}$ at the start of phase one, and this increased to $59.3^{\circ}$ at the end of this phase, with no subsequent change at the end of treatment $\left(\bar{x}=59 \cdot 3^{\circ}\right)$.

In contrast, other cephalometric measures suggest that the mandible achieves a better (i.e., less retrognathic) relationship during phase one. NAP decreased from a mean of $9.5^{\circ}$ to $6.1^{\circ}$ during phase one $(\mathrm{P}<0.0001)$. ANB started at a mean of $5.2^{\circ}$ and decreased to $3.7^{\circ}$ at the end of phase one $(\mathrm{P}<$ 0.0001). ANB continued to decrease during phase two, from a mean of $3.7^{\circ}$ to $2.6^{\circ}(\mathrm{P}=0.01)$. A third measure of maxillomandibular relationship is $\mathrm{AOBO}$, which started at $2.8 \mathrm{~mm}$ and decreased to $0.5 \mathrm{~mm}$ at the end of phase one $(\mathrm{P}=$ 0.0004). AOBO did not change statistically during phase two.

The Facial Angle is of interest because it underwent a significant change during both phases of treatment. The Facial Angle started at a mean of $86.0^{\circ}$, which increased to $86.8^{\circ}$ at the end of phase one $(\mathrm{P}=0.02)$, but then increased to a mean of $88.1^{\circ}$ at the end of treatment $(\mathrm{P}=0.01)$. Overall, this is a net increase of $2.1^{\circ}$ throughout treatment $(\mathrm{P}=0.0001)$.

The maxilla "improved" significantly during phase on (SNA; $\mathrm{P}=0.02)$, with no systematic change during phase two. FMA, the mandibular plane angle, did not change systematically during either phase of treatment. What actually is accomplished during the first (functional) phase of treatment compared to the conventional approach? Based on the cephalometric values we analyzed (Table 6), several skeletal as well as dental changes 
occurred during this phase. Parenthetically, though, several dimensional changes may be attributable to growth, though few changes occur in skeletodental relationships in the absence of treatment (Hamilton et al. 1987).

It may be helpful, as in Table 6, to arrange the variables into three categories of integument, skeleton, and dentition. Regardless, the overall comparison is that more of the overall correction is achieved during phase one than phase two. This is obvious for the two measures of the soft tissue profile (i.e., $\mathrm{Z}$ angle and $\mathrm{E}$ plane), where most of the correction was achieved during phase one.

One likewise sees that most of the skeletal correction occurred during phase one in the Fränkel group (Table 6). The change in AOBO is illustrative. Average $\mathrm{AOBO}$ diminished (i.e., the profile became less retrognathic) by 2.7 mm overall, but most (86\%) of this change was accomplished during phase one. The difference is not as dramatic for NAP and ANB, but again, the majority of the treatment change occurred during phase one. Three of the 10 skeletal variables changed significantly more in phase one of treatment than the other as disclosed by the repeated-measure ANOVA listed to the right in Table 6 . One, $\mathrm{AOBO}$ changed significantly more during phase one $(86 \%$ of total change). Indeed, the improvement in AOBO with fixed appliances (phase two), was not significant $(P=0.49)$, perhaps because most of the desired correction had already been achieved. Two, mandibular length (Condylion-Gnathion) 
increased significantly during both phases, but significantly more so during phase one. It is worth looking at this issue of mandibular growth in a bit more detail. Several investigators have contended that a Fränkel and other functional devices can enhance mandibular growth (see Meikle 2006 for a current review). The study design used in the present study does not address this topic. Phase one and two were both of about 2.5 years duration, but the annualized growth velocity during phase one averaged $3.1 \mathrm{~mm} /$ year which is somewhat higher than the $2.0 \mathrm{~mm} /$ year recorded during phase two $(\mathrm{t}=1.9 ; \mathrm{P}=$ 0.07 by paired $t$-test). The point to be made here is that the much-greater increase of Condylion-Gnathion seen in Table 6 compared to that in phase two should not be interpreted to mean that the Fränkel appliance enhanced growth. We cannot, in fact, decide either way given our study design. The rates of growth are not significantly different during phase one and two, but, of course, patients were older (and, probably, slower growing) during the second phase. The picture is different for Condylion-B Point. The annualized rate of Condylion-B Point growth averaged $4.1 \mathrm{~mm} /$ year during phase one, which is significantly higher than the $1.1 \mathrm{~mm} /$ year documented during phase two (paired $\mathrm{t}=2.6 ; \mathrm{P}=0.015$ ). Again, though, without a matched control group, it is not indisputable that the faster rate of growth during the Fränkel phase was produced by age-appropriate growth alone. 
Table 6 also shows that more of the dental changes occurred during phase one than two. Inspection shows that the nature of the dental changes falls into three categories. Some variables, such as IMPA and FMIA, did not change systematically at all during the course of treatment. Others, like L1 to $\mathrm{AP}$ and L1 to NB, changed significantly during the Fränkel phase and then were changed more (in the same direction) during the fixed appliance phase. For example, the $\mathrm{L} 1$ to $\mathrm{AP}$ angle was increased (i.e., proclinated) about $4^{\circ}$ during phase one and proclined another $3^{\circ}$ during phase two.

As a third group, six of the 13 dental variables disclose changes in opposite directions between the two phases, which probably reflects differences in designs of the two sorts of appliances. These changes are seen in (1) the occlusal plane to Frankfort Horizontal, (2) interincisal angle, (3) U1 to Sella-Nasion, (4) U1 to the Nasion-A Point line (degrees), and (5) U1 to the Nasion-A Point line (mm). Notice that these differences also are disclosed by the repeated-measure ANOVA tests, where each of the five phase-one versus phase-two differences is highly significant statistically.

The occlusal plane (Downs) experienced a nonsignificant increase of $1^{\circ}$ during phase one, but then was decreased about $2^{\circ}$ with fixed appliances. The interincisal angle increased $4^{\circ}$ during phase one, mostly due to uprighting of the upper incisors, but then decreased $2.5^{\circ}$ with fixed appliances. The U1 to Sella-Nasion angle decreased $5.5^{\circ}$ with Fränkel wear (a significant decrease), 
and then increased nonsignificantly during phase two. Comparably, U1 to the Nasion-A Point line (both angular and millimetrically) was reduced significantly with Fränkel wear, but then increased a bit during phase two.

\section{$\underline{\text { Dentition }}$}

Eleven dental variables were measured (Table 6), and nine of these exhibited a significant change during one or the other phases of Fränkel treatment. All of these variables measure positions and angulations of the central incisors.

The maxillary central incisor was uprighted and retracted during phase one which increased the interincisal angle. U1-Sella became more upright, moving back distal of the Nasion-A Point reference line. The mandibular incisor was proclined during phase one treatment. For example, L1 to the A-P line started at $18.4^{\circ}$ and increased to $22.2^{\circ}$ at the end of phase one $(\mathrm{P}=0.0003)$. L1 to AP further increased during the second phase, from a mean of $22.2^{\circ}$ to $25.5^{\circ}(\mathrm{P}=0.004)$

Table 6 discloses four variables where the direction of dental change during phases one and two are in opposite directions. One, interincisal angle increased an average of $3.9^{\circ}$ during phase one $(P=0.003)$, but then decreased $2.5^{\circ}$ during phase two $(\mathrm{P}=0.006)$. Overall, the net change was an increase of $1.4^{\circ}$, which is nonsignificant $(\mathrm{t}=0.7 ; \mathrm{P}=0.47)$. Two, the angle $\mathrm{U} 1$ to Se-Na 
decreased significantly from $106.4^{\circ}$ to $100.6^{\circ}(\mathrm{P}<0.0001)$, but then increased to an average of $103.1^{\circ}$ at the end of treatment, which is a smaller but also significant change statistically $(P=0.0002)$. Overall, the net change in U1 to SeNa averages a decrease of $3.3^{\circ}$, which is significant statistically $(\mathrm{P}=0.04)$.

Three, U1 to the Nasion-A Point line diminished from a mean of $25.6^{\circ}$ to $20.9^{\circ}$ during phase one $(\mathrm{P}<0.0001)$, but then increased to $23.5^{\circ}$ at the end of treatment, which is a significant change of $2.4^{\circ}(\mathrm{P}=0.0007)$. Overall, the net change of $-2.1^{\circ}$, from $25.6^{\circ}$ to $23.5^{\circ}$ is nonsignificant $(t=1.4 ; \mathrm{P}=0.18)$. The fourth variable is the same, but measured millimetrically. Overall, U1 to Na-APoint (in millimeters) went from $4.2 \mathrm{~mm}$ to $4.9 \mathrm{~mm}$, which is nonsignificant ( $\mathrm{t}=$ $1.0 ; \mathrm{P}=0.33)$. 


\section{CHAPTER V}

\section{DISCUSSION}

The prevalence of Class II malocclusions among children in the United States is approximately 15\% (Kelly and Harvey 1977). Although Class II malocclusions are common, treatment timing has been controversial. One approach used today is to begin treatment during the preadolescent years (i.e., the mixed dentition) with limited treatment goals. This early treatment typically is followed by a second phase of treatment with broader goals in adolescence or after the eruption of the permanent dentition. The common alternative treatment approach is to initiate treatment at a later period in the early complete permanent dentition and forego the early treatment phase altogether.

There has been considerable discussion in the orthodontic literature regarding the biological and clinical advantages and disadvantages of early orthodontic treatment. Some have argued that early treatment has no longterm advantage (Bowman 1998; King et al. 2003; Tulloch et al. 2004). Others argue that early treatment may reduce treatment time and reduce the complexity of the second phase of treatment (Dugoni 2004). The primary aim of the present study was to gain substantive information through cephalometric analysis on the efficacy of Fränkel II treatment in the early mixed 
dentition followed by subsequent full appliance treatment. A matched control sample that received fixed edgewise treatment in a single phase was used as a comparison to determine whether the cephalometric results at the end of treatment were comparable.

\section{Time in Treatment}

The results of the present study showed that the one-phase group had a mean time in treatment of 3.1 years compared to 5.2 years for the two-phase group. Overall treatment time, then, was 2 years longer for the two-phase group. These findings agree with those of Bremen and Pancherz (2002), who studied the efficiency of early and late Class II division 1 treatment. They noted that patients treated exclusively with fixed appliance had a shorter duration than did patients treated with functional appliances or a combination of appliances (38 months for functional appliances and 49 months for a combination). These data also concur with those of Livieratos and Johnston (1995) who studied the benefits of a two-stage Bionator/edgewise regimen in comparison to a conventional one-stage edgewise treatment in a "matched" Class II sample. They found that the two-phase group started earlier and finished later. More specifically, the two-phase group was in treatment 1.5 years longer than the one-phase group. Intuitively one would assume that two phases of treatment takes a longer time than a single phase of treatment. 
Livieratos and Johnston (1995) also observed that in addition to taking 18 months longer, the two-stage treatments averaged 10 more appointments. However, the overall appointment rate (13 per year) was lower than for the single-phase edgewise group (17 per year). This difference was attributed to fewer appointments or a more "leisurely" pace during the functional phase of treatment. Similar findings also were noted by Tulloch et al. (2004). In the current study, Fränkel patients were seen every 2 months with minor chair time per appointment during phase one. Both studies revealed no significant difference in treatment time of phase two treatment.

It would seem logical that if early treatment reduced the complexity of later treatment, there should be a reduction in phase two treatment time and/or a need for extraction. In a study of early Class II treatment outcomes, Tulloch et al. (2004) noted that comprehensive treatment took as long in children who had early treatment than in those who did not. They also noted that early treatment did not reduce the need for extractions and/or surgery. In the present study, overall time spent in fixed appliances for the two-phase group was 6 months shorter than in the one-phase treatment. In addition, the two-phase group had a much lower premolar extraction percentage of $3 \%$ ( $1 / 32$ cases) for two-phase group and 56\% (18/32 cases) for one-phase group. Complexity of treatment appears to have been reduced in our two-phase 
subjects considering they had fewer extractions and the second phase of treatment was reduced.

It is unclear how to evaluate the preservation of the four premolars in the two-phase sample. Two-phase treatment substantially increases chances that a case can be treated nonextraction, but so what? Preservation of a full dentition does not seem to affect masticatory efficiency and, of course, the extracted teeth in the midarch do not seem to affect the person's smile or esthetics (Boley and others). Other than the bias that extractions are "wrong" and parents may want their children to be treated without extractions, there seems to be no scientific basis for preferring the retention of all premolars.

\section{Patterns of Skeletodental and Dentoalveolar Change}

During the first phase treatment in the two-phase group, most of the variables tested (16/21) exhibited significant changes. The data suggest that the mandible achieved a less retrognathic relationship during phase one. NAP decreased on average $3.4^{\circ}$, ANB was reduced on average $1.5^{\circ}$, and $\mathrm{AOBO}$ decreased on average $2.3 \mathrm{~mm}$. Tulloch et al. (2004) observed a mean annualized reduction in ANB of $0.91^{\circ}$ for early Class II treatment with the Bionator. Janson and coauthors (2004) compared the treatment effects of the Fränkel appliance on Class II subjects to an untreated control, and they observed a mean reduction in $\mathrm{ANB}$ of $1.2^{\circ}, \mathrm{NAP}$ of $2.5^{\circ}$, and $\mathrm{AOBO}$ of $1.1 \mathrm{~mm}$. These findings 
are in agreement with the present study in that the three important variables that measure maxillomandibular relationship (i.e., $\mathrm{NAP}, \mathrm{ANB}$, and $\mathrm{AOBO}$ ) improved with Fränkel II treatment. Ghafari and coauthors (1998) compared headgear and Fränkel treatment of subjects with Class II division 1 malocclusions. There was an average reduction in ANB of $1.3^{\circ}$ and an average reduction in $\mathrm{AOBO}$ of $2.4 \mathrm{~mm}$ in the Fränkel group. These results are also very similar to our current findings. Toth and McNamara (1999) noted similar reductions in $\mathrm{ANB}$ of $1.1^{\circ}$ in $\mathrm{ANB}$ and $2.2 \mathrm{~mm}$ in $\mathrm{AOBO}$ in their study comparing Fränkel II treatment to untreated Class II controls. McNamara et al. (1990) and Almeida et al. (2002) observed similar ANB reductions of $1.7^{\circ}$ and $0.8^{\circ}$

Of note, though, the one-phase treatment group had similar improvement in skeletal relationships between the two jaws, namely NAP, ANB, AOBO, and the Facial Angle (Table Q5). All of these conditions changed significantly in a favorable direction (i.e., less retrognathic) with treatment. NAP decreased an average of $4.2^{\circ}$ in the present study, ANB decreased $1.5^{\circ}$, AOBO diminished $3.1 \mathrm{~mm}$, and the Facial Angle increased $1.1^{\circ}$.

As with the two-phase changes, these improvements are the result of some combination of growth and treatment. Battagel (1990) examined the relationship between hard and soft tissue changes after treating Class II division 1 malocclusions using edgewise and Fränkel appliance techniques. 
Thirty-two patients were treated non-extraction with a Fränkel appliance, and the remainder by standard edgewise involving extractions. The Fränkel treatment group had a mean reduction in ANB of $1.5^{\circ}$ which was significant, with most of the correction resulting from an SNB increase of $1.0^{\circ}$. Conversely, the standard edgewise group had a greater mean decrease in ANB of $2.3^{\circ}$. However, in contrast to the Fränkel group, the majority of correction was obtained by an SNA reduction of $2.4^{\circ}$. In the current study, ANB reduction in the one-phase sample was largely attributed to a reduction in SNA $\left(-1.3^{\circ}\right)$ with a trivial increase in SNB of $0.2^{\circ}$. This result is in agreement with Battagal (1990). However, in the two-phase Fränkel sample the majority of ANB correction involved a reduction in SNA $\left(-1.0^{\circ}\right)$ with a smaller SNB increase of $0.4^{\circ}$ which is in contrast with the findings of Battagal (1990), Hamilton et al. (1987), Ghafari et al. (1998), Toth and McNamara (1999), and Janson (2004), all of whom all observed a greater increase in SNB with treatment and smaller reductions in SNA during Fränkel treatment. One plausible explanation for this finding may be that some of the two-phase sample received headgear therapy during phase one in the present study. The clinician stated that supplemental headgear was often used in concert with Fränkel therapy during phase one. This may explain the larger-than-normal reduction in SNA seen in the present study. 
Luppanapornlarp and Johnston (1993) studied the effects of premolar extractions on outcomes of extraction and nonextraction patients with Class II malocclusions. All subjects had edgewise appliances as part of the treatment regimen. Cephalometric data from the study revealed that ANB was reduced an average of $1.8^{\circ}$ in the extraction group and $1.0^{\circ}$ in the nonextraction group, with the majority of correction achieved with a reduction in SNA $\left(-1.9^{\circ}\right.$ for extraction and $-1.4^{\circ}$ for nonextraction). AOBO also reduced in a favorable direction with an average reduction of $-1.9^{\circ}$ for the extraction group and $-1.1^{\circ}$ for the nonextraction group, which is similar to the current study.

Nine of eleven dental variables (Table 4) exhibited significant changes during treatment in the Fränkel group. In general, the maxillary central incisor was uprighted and retracted during phase one, resulting in an increased interincisal angle. Conversely, the mandibular incisor was proclined during phase one. Janson and coworkers (2004) observed similar dental movements during Fränkel II treatment. The Fränkel group had significant palatal tipping and a decrease in protrusion of the maxillary incisors compared with the control group (e.g., Upper 1-NA reduced $8.0^{\circ}$ and overjet reduced $3.8 \mathrm{~mm}$ ). Toth and McNamara (1999) observed a reduction of Upper 1-SN of 3.3 during Fränkel II treatment with an increased IMPA of $1.1^{\circ}$. Almeida et al. (2002) and Ghafari et al. (1998) observed similar dental movements, with a reduction in Upper 1-NA of $4.8^{\circ}$ and $5.7^{\circ}$, and an increase in Lower $1-\mathrm{NB}$ of $2.7^{\circ}$ and $3.1^{\circ}$, 
respectively. Uprighting the maxillary incisors and proclining the mandibular incisors would appear to be beneficial to subjects with large overjets and accentuated tipping of the maxillary incisors, which are characteristic of Class II division 1 malocclusions. In our Fränkel sample, overjet was reduced by 3.3 $\mathrm{mm}$ during phase one, in part due to these dental movements.

Similar dental movements were observed for the one-phase subjects. In general, the maxillary incisor was retracted (Upper 1-SN was reduced $2.2^{\circ}$, Upper 1-NA was reduced $1.0^{\circ}$ ) while the mandibular incisor was proclined (IMPA increased $2.73^{\circ}$, Lower 1-NB increased $2.29^{\circ}$, and FMIA decreased $\left.1.42^{\circ}\right)$. Once again these dental movements would aid in overjet correction.

\section{End of Treatment}

How comparable the skelotodental variables are at the end of treatment is of prime importance in the present study. Of the eight skeletal variables only ANB and FMA differed significantly. The FMA of the two-phase group was higher by $1.8^{\circ}$, while ANB was $1.3^{\circ}$ less than the one-phase group. The use of the Fränkel appliance was effective in modifying growth during the early phase of treatment (i.e., NAP decreased on average $3.4^{\circ}$, ANB was reduced on average $1.5^{\circ}$, and $\mathrm{AOBO}$ decreased on average $2.3 \mathrm{~mm}$ ). However, at the end of comprehensive fixed appliance therapy for all subjects the cephalometric data were very similar. This finding is in agreement with Tulloch et al. (2004), Dolce 
et al. (2005), and O'Brien et al. (2005) whose data disclose comparable changes. Two-phase orthodontic therapy appears to be less efficient than a single phase treatment in that it involves more treatment time with a similar posttreatment result. One striking difference between the present study and Tulloch et al. (2004) is the number of subjects requiring premolar extraction. Tulloch and coauthors (2004) concluded that no difference existed among previously treated and untreated groups with regards to the number of subjects requiring premolar extractions during comprehensive treatment. In the present study, the majority of two-phase subjects were treated without the removal of premolars, while the majority of one-phase subjects had extractions. What our study shows is that similar skeletodental endpoints can be achieved with the preservation of teeth. We have no opinion on the benefit, if any, that four premolars have on esthetics or function. A common criticism of nonextraction treatment is that it is more unstable; however, our two-phase sample and onephase sample had very similar skeletodental relationships at the end of treatment. Presumably, our two-phase sample is no less stable than the onephase group, though, this is beyond the scope of this study. What can be concluded is that both treatment alternatives can arrive at a desirable endpoint (i.e., Class I canine, overjet, overbite, and similar skeletodental variables). 


\section{CHAPTER VI \\ SUMMARY AND CONCLUSIONS}

There has been considerable discussion in the orthodontic literature regarding the biological and clinical advantages and disadvantages of "early" orthodontic intervention, specifically for Class II malocclusions. Of primary concern is the benefit, or lack thereof, gained with regard to treatment outcomes when using a two-phase treatment protocol compared to a one-phase treatment approach. The present study compared two groups of patients, one treated with the Fränkel II appliance in the mixed dentition followed by full appliance treatment. The second group was treated in a single phase with conventional edgewise mechanics. The question was whether the cephalometric results at the end of treatment were comparable.

This study was a retrospective analysis of cephalograms from patients with Class II, division 1 malocclusions at the start of treatment. These were 32 consecutively treated patients who received phase 1 (early) treatment with a Fränkel II appliance and later with full appliances (all treated by a single clinician). The control group consisted of a sample of subjects with similar Class II, division 1 malocclusions who were treated in a single phase with fixed edgewise appliances only. Both treatment groups consisted of 32 subjects, each of which were matched based on sex and cephalometric values. 
The results of the present study showed that the one-phase group had a mean time in treatment of 3.1 years compared to 5.2 years for the two-phase group. Overall treatment time, then, was two years longer for the two-phase group. However, the two-phase group was in full fixed appliances on average 6 months less than the one-phase edgewise sample. In addition, the two-phase group had a much lower premolar extraction percentage of $3 \%(1 / 32)$ for the two-phase group and 56\% (18/32) for the one-phase group. This indicated that the full appliance phase in the two-phase group was less complex on average.

In terms of profile changes, neither integumental variable ( $\mathrm{Z}$ angle, $\mathrm{E}$ plane) differed statistically. Both variables were more desirable in the onephase group, showing that the lower lip is less retrusive. Again, though, neither variable truly differed statistically. Skeletally, ANB was greater in the one-phase sample. That is, ANB was significantly smaller in the two-phase sample $\left(\bar{x}=2.6^{\circ}\right)$ than the one-phase sample $\left(\bar{x}=4.0^{\circ}\right)$. The mandibular plane angle also differed at the end of treatment. The two-phase sample had a final FMA which was $1.8^{\circ}$ steeper than the one-phase sample. Dentally, the maxillary and mandibular incisors in both treatment groups proclined during treatment. In conclusion, quite similar skeletodental endpoints were achieved regardless of which treatment protocol was employed. By these outcome criteria, the treatments can be viewed as equivalent approaches to a common problem. What the study shows is that, for the average Class II patient, 
intervention with a Fränkel II followed by fixed appliance treatment yields

effectively the same skeletodental outcome as conventional edgewise

treatment, though some patients treated with conventional edgewise may not have as many premolars at the end of treatment. 
LIST OF REFERENCES 
Almeida MR, Henriques JF, Ursi W. Comparative study of the Fränkel (FR-2) and bionator appliances in the treatment of Class II malocclusion. Am J Orthod Dentofacial Orthop 2002;121:458-66.

Arya BS, Savara BS, Thomas DR. Prediction of first molar occlusion. Am J Orthod 1973;63:610-21.

Baccetti T, Franchi L, McNamara JA Jr, Tollaro I. Early dentofacial features of Class II malocclusion: a longitudinal study from the deciduous through the mixed dentition. Am J Orthod Dentofacial Orthop 1997;111:502-9.

Balters W. Die Einfugrund in die Bionatorheilmethode. In: Ausgewahlte, Schriften und Vortraage, Druckerei Holzer, Heidelbert, 1973.

Balters W. Die Technik und Ubung der allgemeinen and speziellen bionatortherapie. Quintessenz, 1964;1:77.

Battagel JM. The relationship between hard and soft tissue changes following treatment of Class II division 1 malocclusions using Edgewise and Fränkel appliance techniques. Europ J of Orthod 1990;12:154-65.

Baume LJ. Physiological tooth migration and its significance for the development of occlusion; the biogenesis of accessional dentition. J Dent Res 1950;29:440-7.

Behrents RG. An atlas of growth in the aging craniofacial skeleton, Monograph number 18. Craniofacial Growth Series. Ann Arbor: Center for Human Growth and Development, The University of Michigan, 1985. 
Berg R. Post-retention analysis of treatment problems and failures in 264 consecutively treated cases. Eur J Orthod 1979;1:55-68.

Bishara SE. Mandibular changes in persons with untreated and treated Class II Division 1 malocclusions. Am J Orthod Dentofacial Orthop 1998;113:661-73.

Bishara SE, Hoppens BJ, Jakobsen JR, Kohout FJ. Changes in the molar relationship between the deciduous and permanent dentitions: a longitudinal study. Am J Orthod Dentofacial Orthop 1988;93:19-28.

Björk A. The principle of the Andresen method of orthodontic treatment: a discussion based upon cephalometric X-ray analysis of treated cases. Am J Orthod 1951;37:437-58.

Boley JC, Pontier JP, Smith S, Fulbright M. Facial changes in extraction and nonextraction patients. Angle Orthod 1998;68:539-46.

Bolmgren GA, Moshiri F. Bionator treatment in Class II, division 1. Angle Orthod 1986;56:255-62.

Bowman SJ. One stage versus two-stage treatment: are two really necessary? Am J Orthod Dentofacial Orthop 1998;113:111-6.

Breiden CM, Pangrazio-Kulbersh V, Kulbersh R. Maxillary skeletal and dental change with Fränkel appliance therapy - an implant study. Angle Orthod 1984;54:232-66. 
Bremen J, Pancherz H. Efficiency of early and late Class II Division 1 treatment. Am J Orthod Dentofacial Orthop 2002;121:31-7.

Brodie AG. On the growth pattern of the human head. Am J Anat 1941;68:20962.

Chadwick SM, Aird JC, Taylor PJ. Functional regulator treatment of Class II division 1 malocclusions. Eur J Orthod 2001;23:495-505.

Creekmore TD, Radney LJ. Fränkel appliance therapy: orthopedic or orthodontic? Am J Orthod 1983;83:89-108.

Dann C IV, Phillips C, Broder H, Tulloch C. Self-concept, Class II malocclusion, and early treatment. Angle Orthod 1995;65:411-16.

Diers N. The functional regulator of Fränkel. Clarks Clinical Dentistry. Vol. 2. Philadelphia, Pa: JB Lippincott, 1998;25:1-25.

Dolce C, Schader RE, McGorray SP, Wheeler TT. Centrographic analysis of 1phase versus 2-phase treatment for Class II malocclusion. Am J Orthod Dentofacial Orthop 2005;128:195-200.

Dugoni SA. Comprehensive mixed dentition treatment: can it avoid full banded therapy? [Summary of presentation to annual meeting, September 30, 1996, Monterey, California]. Pac Coast Soc Orthod Bull 1996;68:33-4. 
Dugoni SA, Aubert M, Baumrind S. Differential diagnosis and treatment planning for early mixed dentition malocclusions. Am J Orthod Dentofacial Orthop 2006;129:S80-1.

Durkin JF. Secondary cartilage: a misnomer? Am J Orthod 1983:83;89-108.

Elsasser WA, Wylie WL. The craniofacial morphology of mandibular retrusion. J Anthrop 1943;6:461-73.

Enlow DH. The human force: an account of the postnatal growth and development of the craniofacial skeleton. New York: Harper and Row, 1968, p 110.

Falck F. Kephalometrische Längsschnittuntersuchung uber Behandlungsergebnisse der mandibularen Retrognathie mit Funktionsreglen im Vergleich zu einer Controllgruppe. Med. Diss., Berlin, 1985.

Fränkel R. The artificial translation of the mandible by function regulators. In: J.T. Cook (ed). Transaction of the Third International Orthodontic Congress. St. Louis: Mosby Company, 1975.

Fränkel R. Decrowding during eruption under the screening influence of vestibular shields. Am J Orthod 1974;65:372-406.

Fränkel R. A functional approach to orofacial orthopedics. Br J Orthod 1980;7:41-51. 
Fränkel R. Guidance of eruption without extraction. Trans Eur Orthod Soc 1971, p 303-15.

Fränkel R. The theoretical concept underlying treatment with functional correctors. Trans Eur Orthod Soc 1966, p 223-50.

Fränkel R, Fränkel C. Orofacial orthopedics with the function regulator. S. Karger, Munich, 1989.

Fränkel R, Fränkel C. A rejoinder. Am J Orthod Dentofacial Orthop 987;92:425536.

Fröhlic FJ. A longitudinal study of untreated Class II type malocclusions. Trans Eur Orthod Soc 1961;37:137-59.

Ghafari J, Shofer FS, Hunt J, Markowitz DL, Laster LL. Headgear versus function regulator in the early treatment of Class II, Division 1 malocclusion: a randomized clinical trial. Am J Orthod Dentofacial 1998;113:51-61.

Gianelly AA. One-phase versus two-phase treatment. Am J Orthod Dentofacial Orthop 1995;108:556-9.

Graber TM. Functional appliances. In: Graber TM, Vanarsdall RL, Vig KWL, (eds). Orthodontics: current principles and techniques. St. Louis: Mosby, 2005, p 493-542.

Graber TM, Neumann B. Removable orthodontic appliances. Philadelphia: Saunders, 1984, p 85-90. 
Hamilton SD, Sinclair PM, Hamilton RH. A cephalometric, tomographic, and dental cast evaluation of Fränkel therapy. Am J Orthod Dentofacial Orthop 1987;92:427-34.

Henry RG. A classification of Class II, division 1 malocclusions. Angle Orthod 1957;27:83-92.

Hunter WS. The vertical dimensions of the face and skeletodental retrognathism. Am J Orthod Dentofacial Orthop 1967;53:586-95.

Illing HM, Morris DO, Lee RT. A prospective evaluation of Bass, Bionator and Twin Block appliances. Part I-The hard tissues. Eur J Orthod 1998;20:501-16.

Janson GRP, Toru o JLA, Martins DR. Class II treatment effects of the Fränkel appliance. Euro J Orthod 2003;25:301-9.

Keeling SD, King GJ, Wheeler TT, McGorry S. Timing of Class II treatment: rationale, methods, and early results of an ongoing randomized clinical trail. In: Trotman C, McNamara JA Jr. (eds). Orthodontic treatment: outcome and effectiveness, Monograph 30. Craniofacial Growth Series. Ann Arbor: Center for Human Growth and Development, The University of Michigan, 1995, p 1-112.

Kelly J, Harvey C. An assessment of the teeth of youth 12-17 years. United States. DHEW publication no. (HRA) 77-1644. Washington, DC: National Center for Health Statistics, 1977, p 1-18. 
King GJ, McGorray SP, Wheeler TT, Dolce C, Taylor M. Comparison of peer assessment ratings (PAR) from 1-phase and 2-phase treatment for Class II malocclusions. Am J Orthod Dentofacial Orthop 2003;123:489-96.

King GJ, Wheeler TT, McGorray SP, Aiosa LS, bloom RM, Taylor MG.

Orthodontists' perceptions of the impact of phase 1 treatment for Class II malocclusion on phase 2 needs. J Dent Res 1999;78:1745-53.

Koski K. Cranial growth centers: facts of fallacies? Am J Orthod 1968;54:56683.

Koski K. The role of the craniofacial cartilages in the postnatal growth of the craniofacial skeleton. In: Dahlberg AA, Graber TM, (eds). Orofacial growth and development. Philadelphia: the Hague, 1977, p 9-34.

Livieratos FA, Johnston LE Jr. A comparison of one-stage and two-stage nonextraction alternatives in matched Class II samples. Am J Orthod Dentofacial Orthop 1995;108:118-31.

Luppanapornlarp S, Johnston LE Jr. The effects of premolar-extraction: a long-term comparison of outcomes in "clear-cut" extraction and nonextraction Class II patients. Angle Orthod 63:257-72.

McDougall PD, McNamara JA Jr, Dierkes JM. Arch width development in Class II patients treated with the Fränkel appliance. Am J Orthod $1982 ; 82: 10-22$. 
McNamara JA. Components of Class II malocclusion in children 8-10 years of age. Angle Orthod 1981;51:177-202.

McNamara JA Jr, Brudon WL. Orthodontic and orthopedic treatment in the mixed dentition. Ann Arbor: Needham Press, 1993, p 3-6.

McNamara JA Jr, Connelly TG, McBride MC. Histological studies of temporomandibular joint adaptations. In: McNamara JA Jr (ed). Control mechanisms in craniofacial growth, Monograph 3. Craniofacial Growth Series. Ann Arbor: Center for Human Growth and Development, The University of Michigan, 1975.

McNamara JA Jr, Howe RP, Dischinger TG. A comparison of Herbst and Fränkel treatment in Class II malocclusion. Am J Orthod Dentofacial Orthop 1990;98:134-44.

Meikle MC. Remodeling the dentofacial skeleton: the biological basis of orthodontics and dentofacial orthopedics. J Dent Res 2007;86:12-24.

Mills JR. The effect of functional appliances on the skeletal pattern. J Orthod 1991;18:267-8.

Mills JR, McCulloch K. Posttreatment changes after successful correction of Class II malocclusions with the Twin Block appliance. Am J Orthod Dentofacial Orthop 2000;118:24-33.

Moss ML. Functional cranial analysis of the mandibular angular cartilage of the rat. Angle Orthod 1969;39:209-14. 
Moss ML. The functional matrix. Vistas in orthodontics. Philadelphia: Lea and Febiger, 1962, p 85-98.

Moss ML. Growth of the calvaria in the rat: the determination of osseous morphology. Am J Anat 1954;94:333-41.

Moss ML. The primacy of functional matrices in orofacial growth. Dent Pract 1968;19:65-73.

Moyers RE. Handbook of orthodontics, 4th ed. Chicago: Year Book Medical Publishers, 1988, p 346-7, p 433-4.

Moyers RE, Riolo ML, Guire KE, Wainright RL, Bookstein FL. Differential diagnosis of class II malocclusions. Part 1. Facial types associated with class II malocclusions. Am J Orthod 1980;78:477-94.

Nelson C, Harkness M, Herbisson P. Mandibular changes during functional appliance treatment. Am J Orthod Dentofacial Orthop 1993;104:153-61.

Ngan PW, Byczek E, Scheick J. Longitudinal evaluation of growth changes in Class II division 1 subjects. Semin Orthod 1997;3:222-31.

O'Brien K, Wright J, Conboy F. Effectiveness of early orthodontic treatment with the Twin-block appliance: a multicenter, randomized, controlled trial. Part 1: Dental and skeletal effects. Am J Orthod Dentofacial Orthop 2003;124:234-43. 
O'Brien K, Wright J, Conboy F. Effectiveness of early orthodontic treatment with the Twin-block appliance: a multicenter, randomized, controlled trial. Part 2: Psychosocial effects. Am J Orthod Dentofacial Orthop 2003;124:488-95.

O'Brien K, Wright J, Conboy F. Effectiveness of treatment for Class II malocclusion with the Herbst or Twin-block appliances: a randomized, controlled trial. Am J Orthod Dentofacial Orthop 2003;124:128-37.

Petrovick AG. Control processes in the postnatal growth of the condylar cartilage of the mandible. In: Johnston LE Jr. (ed). Control mechanisms in craniofacial growth. Craniofacial Growth Series. Ann Arbor: Center for Human Growth and Development, The University of Michigan, 1975:101-54.

Petrovick A, Gasson N, Oudet C. Growth Hormone, postural hyperpropulsion and condylar prechondroblast proliferation in rats. J Dent Res 1974:53;745-6.

Petrovick AG, Stutzmann JJ, Gasson N: The final length of the mandible: is it genetically predetermined? Is the functional maxipropulsion involving periodic forward repositioning the best procedure to elicit over lengthening? In: Carlson DS (ed). Craniofacial biology, Monograph 10. Craniofacial Growth Series. Ann Arbor: Center for Human Growth and Development, The University of Michigan, 1981. 
Proffit WR, Fields HW, Moray LJ. Prevalence of malocclusion and orthodontic treatment need in the United States: estimates from the NHANES III survey. Int J Adult Orthognath Surg 1988;13:97-106.

Remmer KR, Mamandras AH, Hunter S, Way DC. Cephalometric changes associated with treatment using the activator, the Fränkel appliance, and the fixed appliance. Am J Orthod 1985;88:363-72.

Renfroe EW. A study of the facial patterns associated with Class I, Class II, and division 1, and Class II, division 2 malocclusions. Angle Orthod 1948;19:12-5.

Ricketts RM. Cephalometric analysis and synthesis. Angle Orthod 1961;31:11541.

Riedel RA. The relation of maxillary structures to the cranium in malocclusion and normal occlusion. Angle Orthod 1952;22:142-5.

Ruf S, Pancherz H. Temporomandibular joint remodeling in adolescents and young adults during Herbst treatment: a prospective longitudinal magnetic resonance imaging and cephalometric radiographic investigation. Am J Orthod Dentofacial Orthop 1999;115:607-18.

Sicher J. Oral anatomy. St. Louis: Mosby Company, 1952.

Smith GC, Pell JP. Parachute use to prevent death and major trauma related to gravitational challenge: systematic review of randomized controlled trials. BMJ 2003;327:1459-61. 
Sokal RR, Rohlf FJ. Biometry: the principles and practice of statistics in biological research, 3rd ed. San Francisco: WH Freeman and Company, 1995.

Stockli PW, Willert HG. Tissue reactions in the temporomandibular joint resulting from anterior displacement of the mandible in the monkey. Am J Orthod 1971;60:142-54.

Toth LR, McNamara JA Jr. Treatment effects produced by the Twin-block appliance and the FR-2 appliance of Fränkel compared with an untreated Class II sample. Am J Orthod Dentofacial Orthop 1999;116:597-609.

Tukey JW. Exploratory data analysis. Massachusetts: Addison-Wesley, Reading, 1977, p 1-56.

Tulloch JFC, Proffit WR, Phillips C. Outcomes in a 2-phase randomized clinical trial of early Class II treatment. Am J Orthod Dentofacial Orthop 2004;125:657-67.

Van der Klaauw CJ. Cerebral skull and facial skull. Arch Neerl Zool 1946;7:1637.

White RC. The role of mandibular growth in occlusal development. Unpublished M.S. Thesis. St Louis University, St. Louis, Missouri, 1983. 
Whitney DJ. A longitudinal morphometric analysis and classification of the Class II individual. Unpublished M.S. Thesis. Case Western Reserve University, Cleveland, Ohio, 1983.

Winer BJ, Brown DR, Michels KM. Statistical principles in experimental design, 3rd ed. New York: McGraw-Hill Book Company, 1991.

Woolf CM. Principles of biometry. Princeton, NJ: D. Van Nostrand Company, Inc., 1968. 


\section{APPENDIX.}

\section{CEPHALOMETRIC TRACINGS}

Prints of the cephalometric tracings (superimpositions) of the subjects used in the present study. For the Fränkel-treated group, the black lines are at pretreatment, the green lines are at the beginning of fixed appliances, and the red lines are at the end of treatment. For the edgewise cases, the black lines are at the start of treatment, and the red lines are at the end of treatment. All tracings are printed at true size (i.e., 1:1 with the original radiograph). 


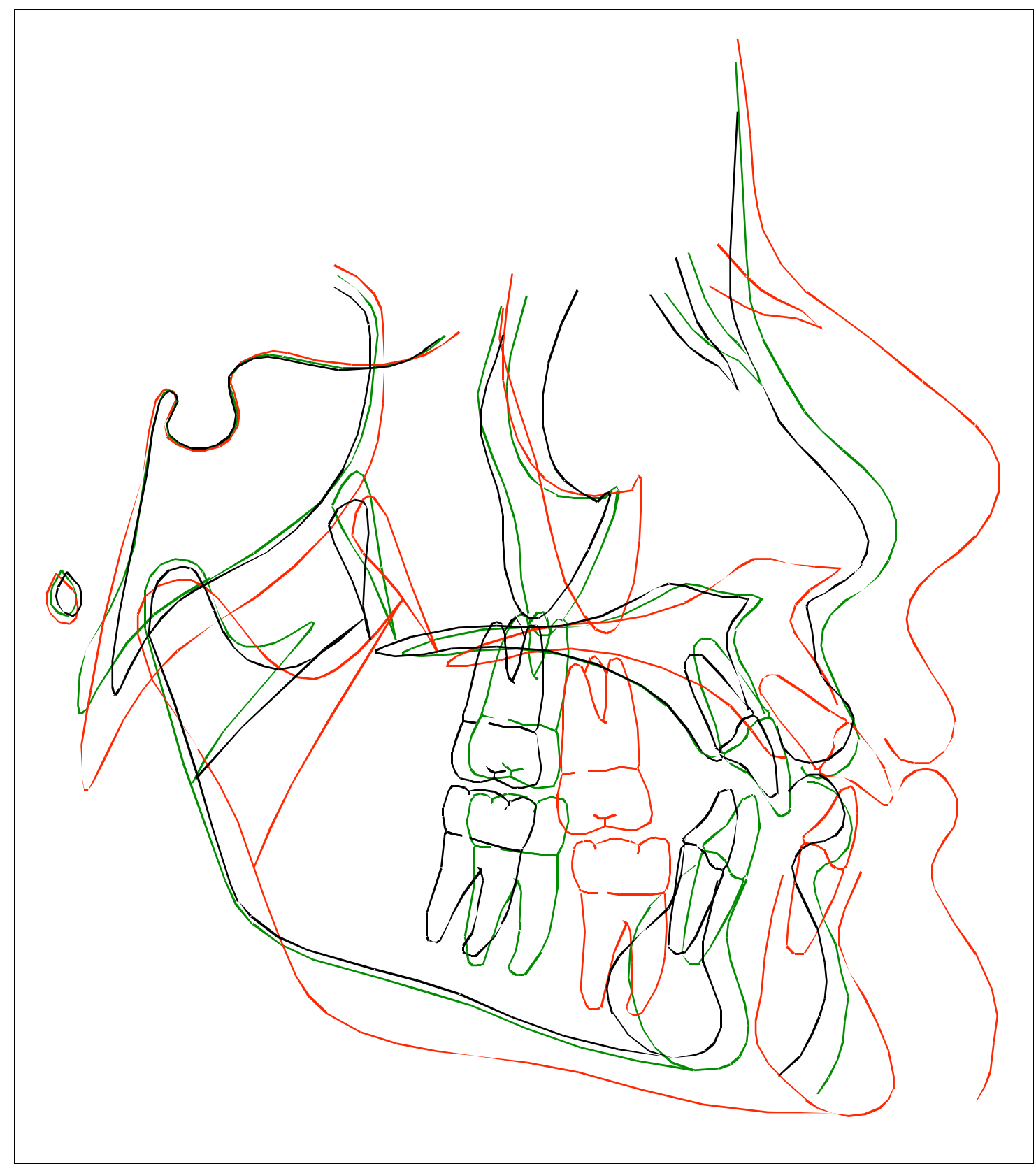

Figure A-1: Print of cephalometric tracing superimposition subject 01 . 


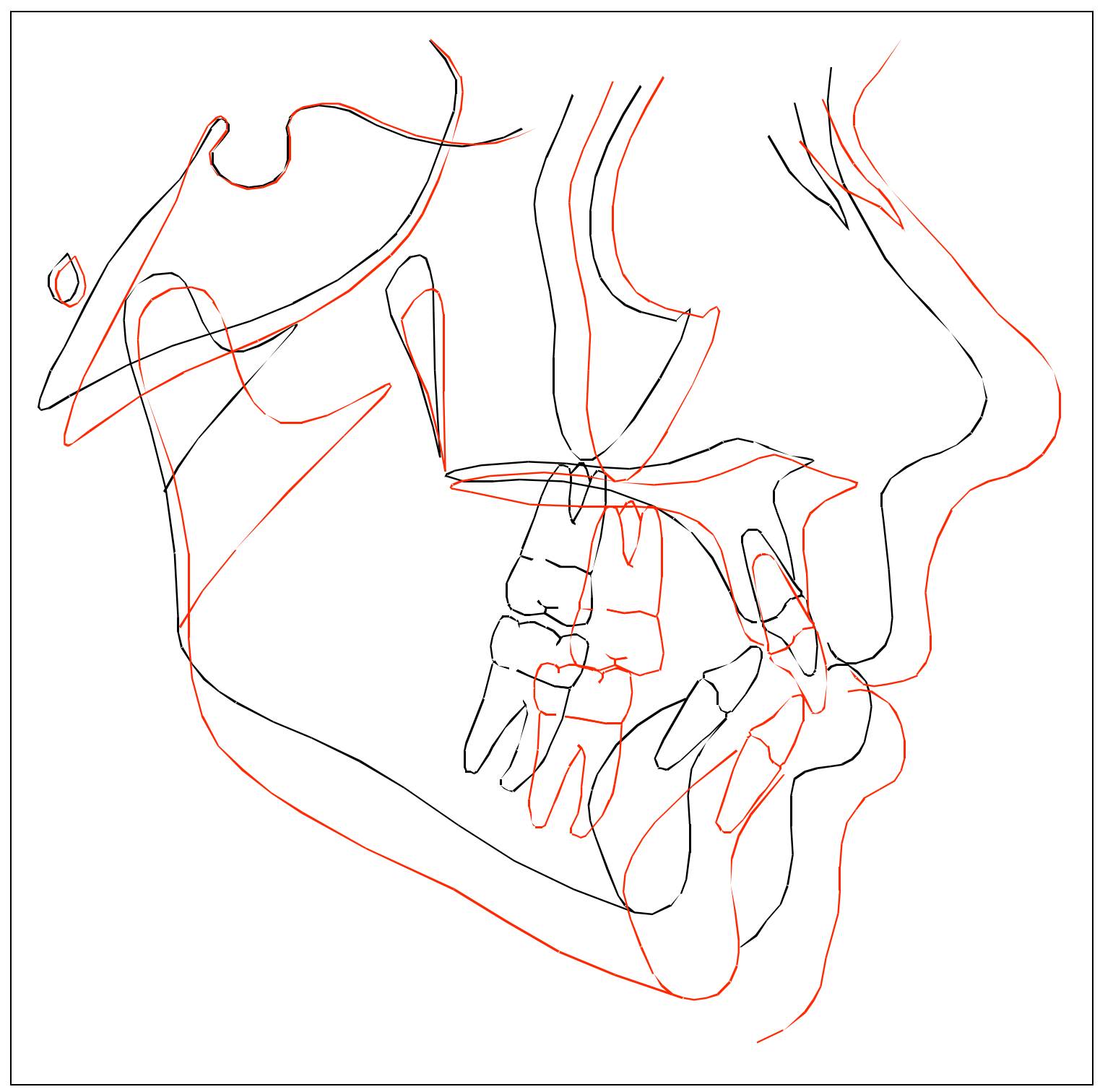

Figure A-2: Print of cephalometric tracing superimposition subject 02 . 


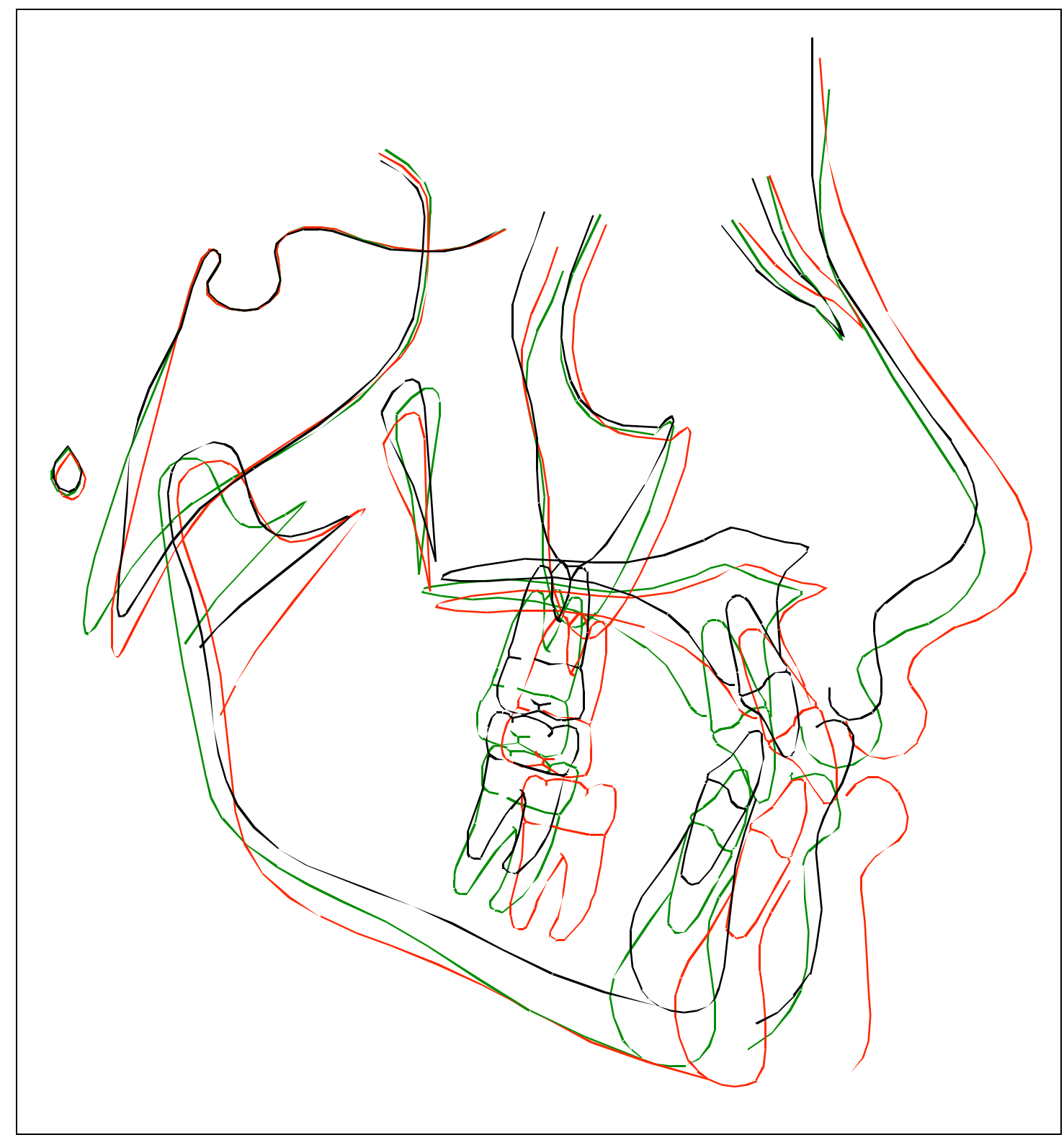

Figure A-3: Print of cephalometric tracing superimposition subject 03. 


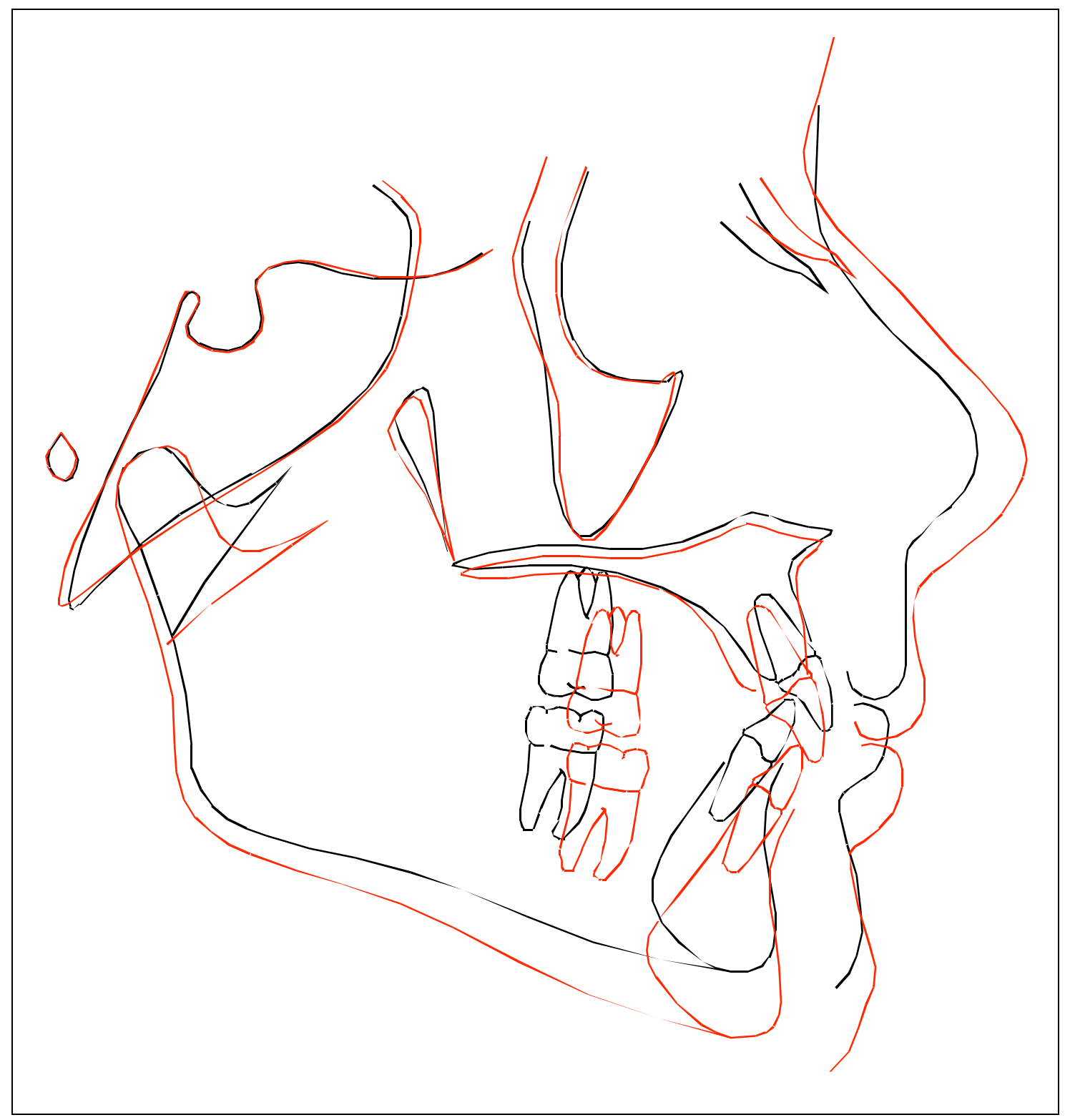

Figure A-4: Print of cephalometric tracing superimposition subject 04. 


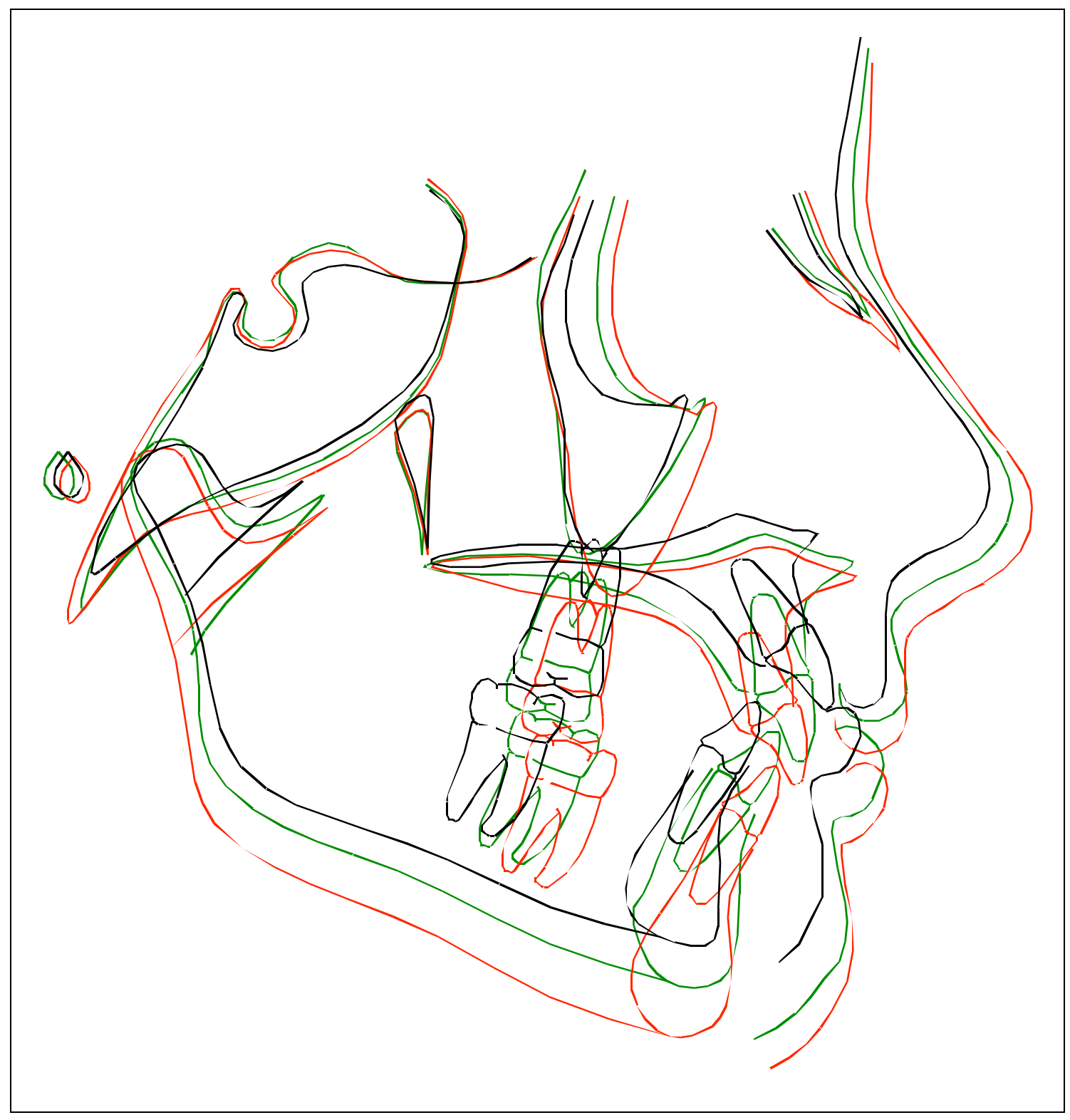

Figure A-5: Print of cephalometric tracing superimposition subject 05. 


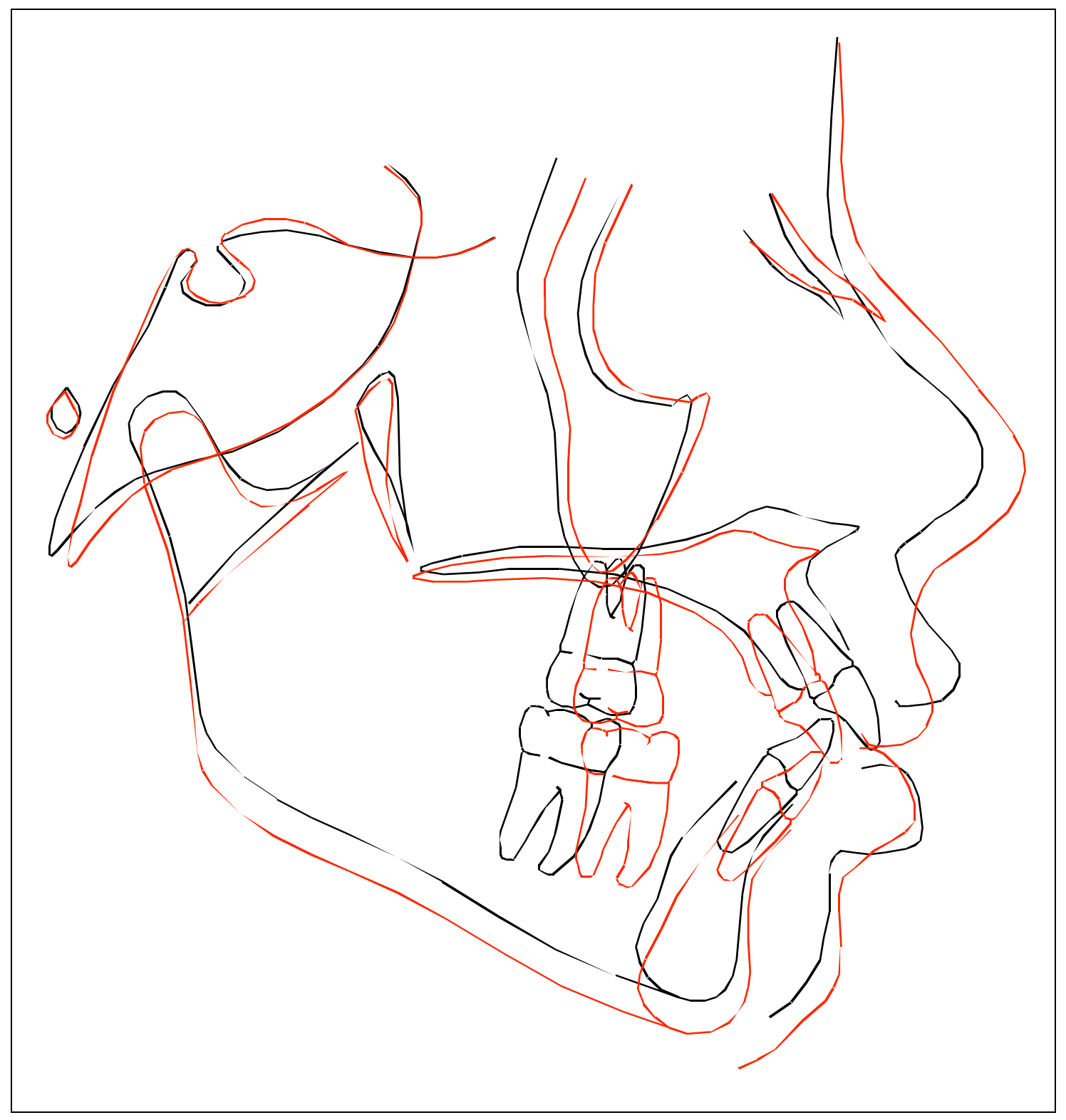

Figure A-6: Print of cephalometric tracing superimposition subject 06. 


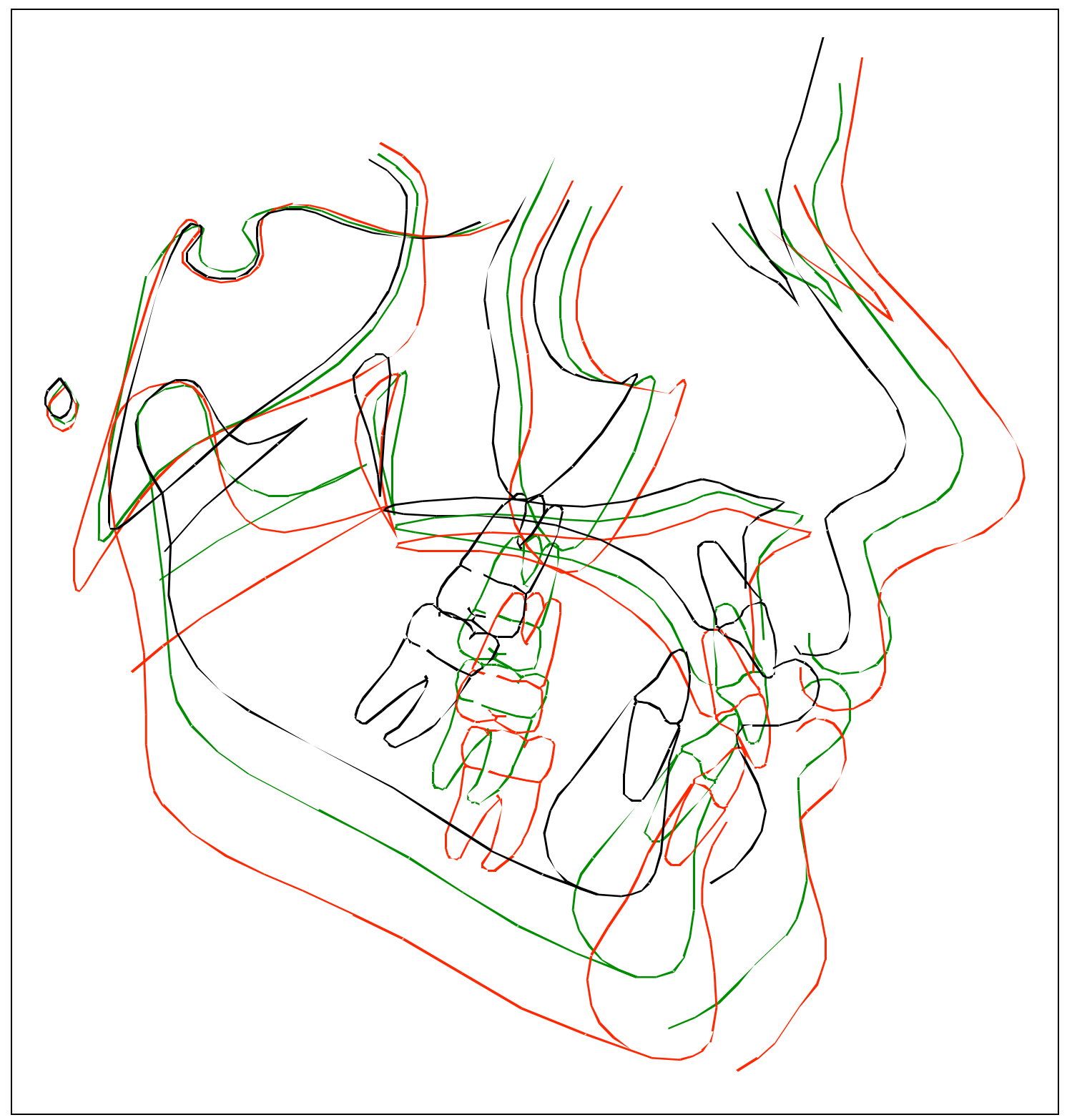

Figure A-7: Print of cephalometric tracing superimposition subject 07. 


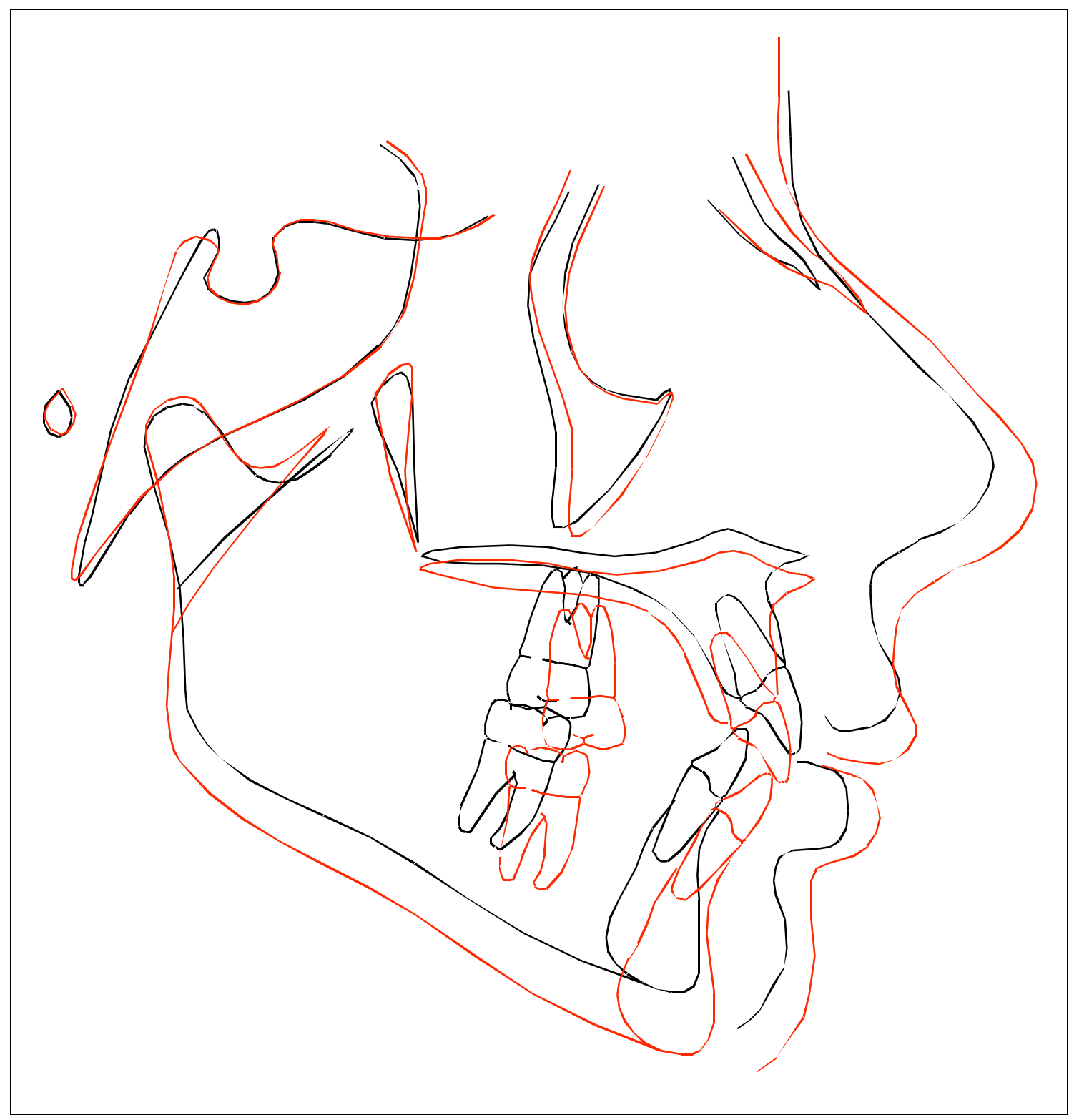

Figure A-8: Print of cephalometric tracing superimposition subject 08 . 


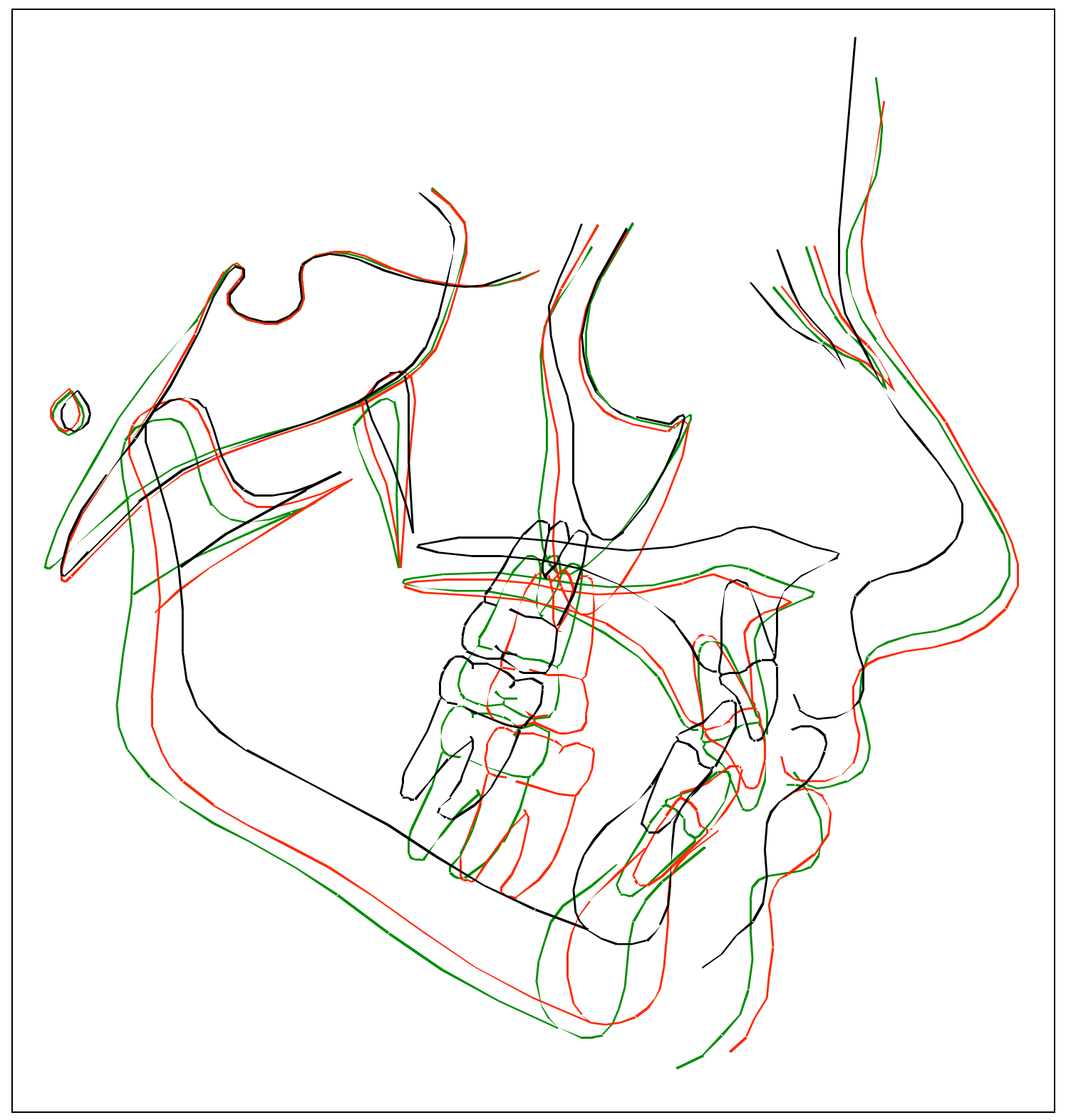

Figure A-9: Print of cephalometric tracing superimposition subject 09. 


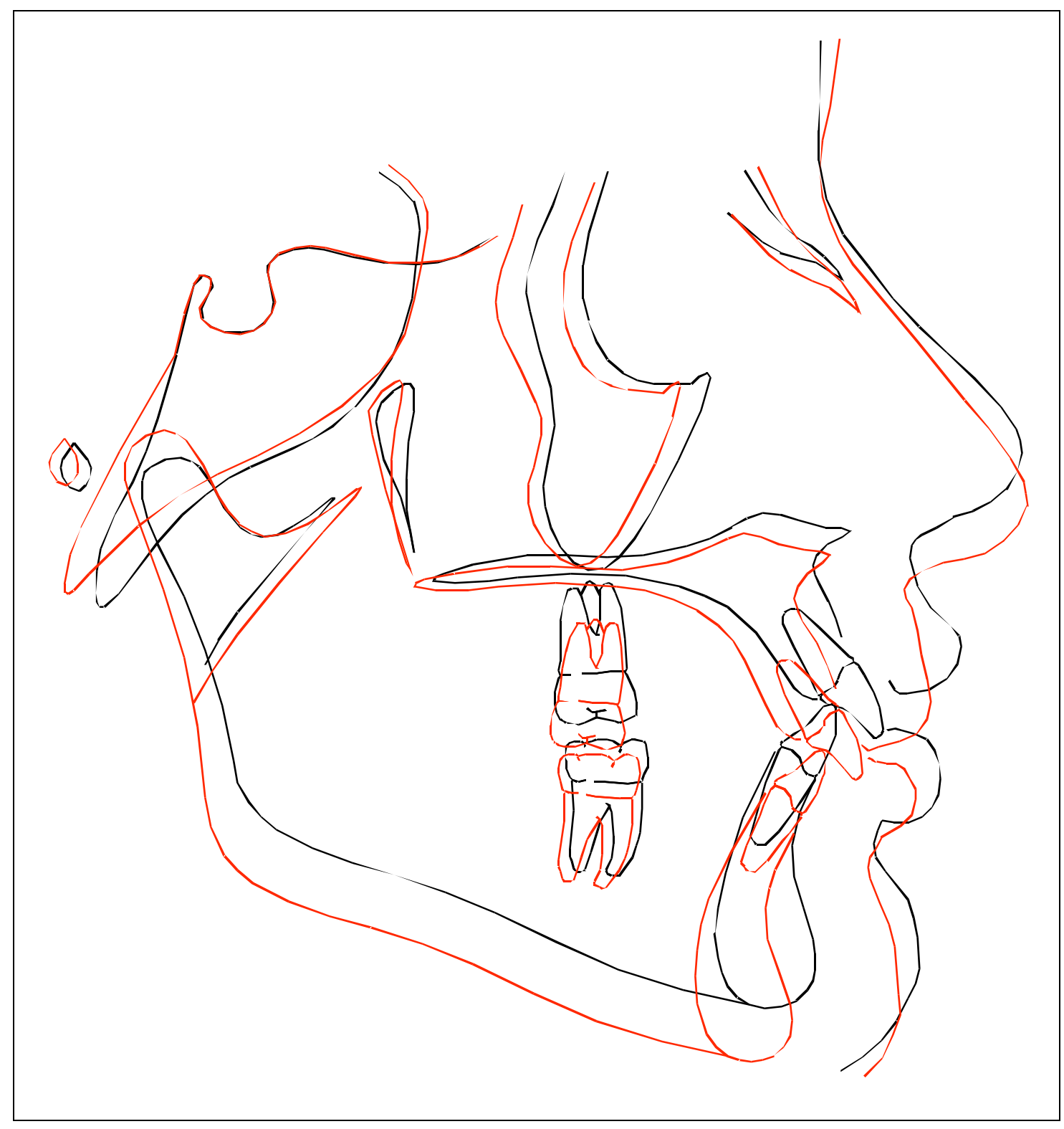

Figure A-10: Print of cephalometric tracing superimposition subject 10. 


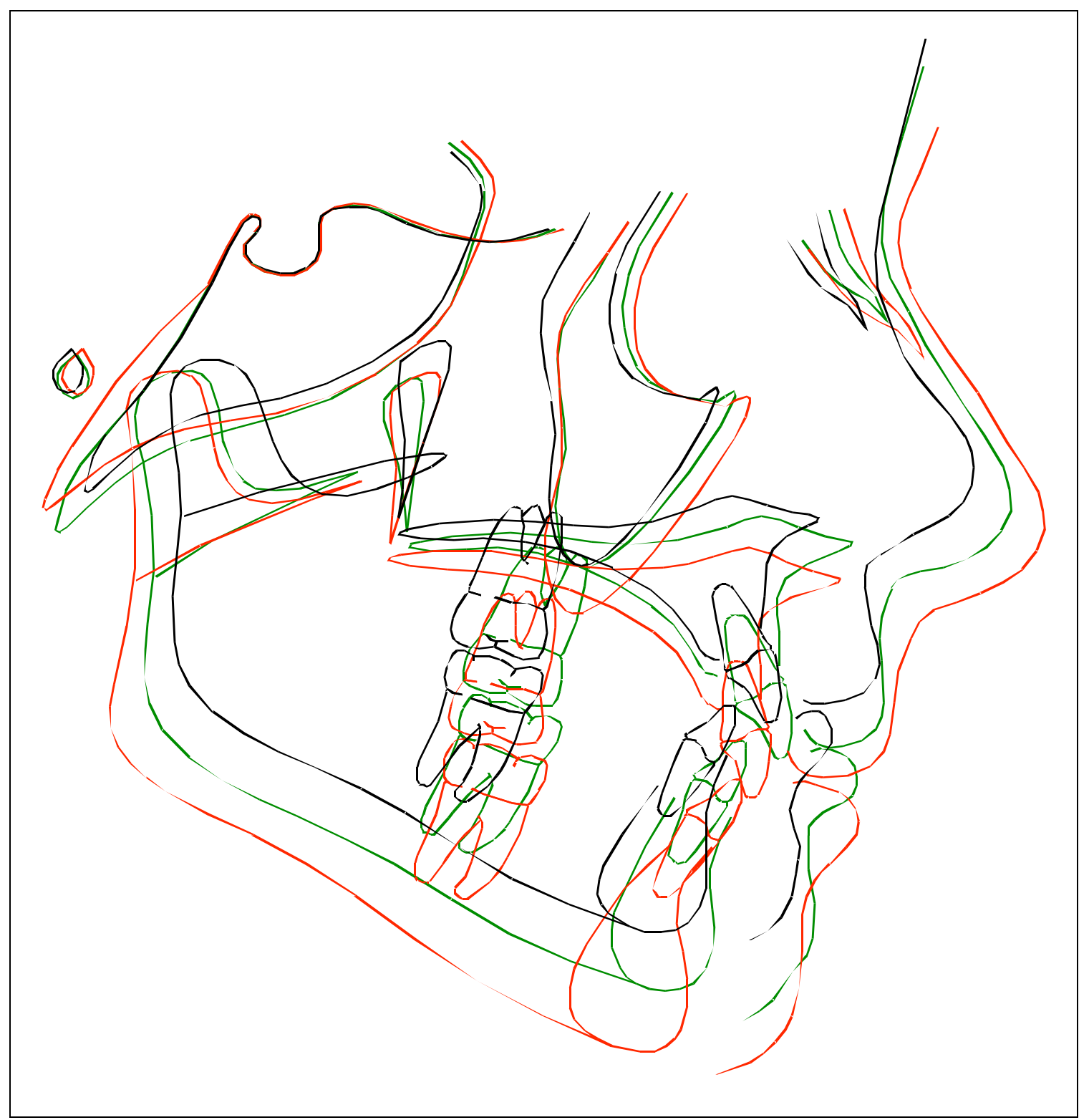

Figure A-11: Print of cephalometric tracing superimposition subject 11. 


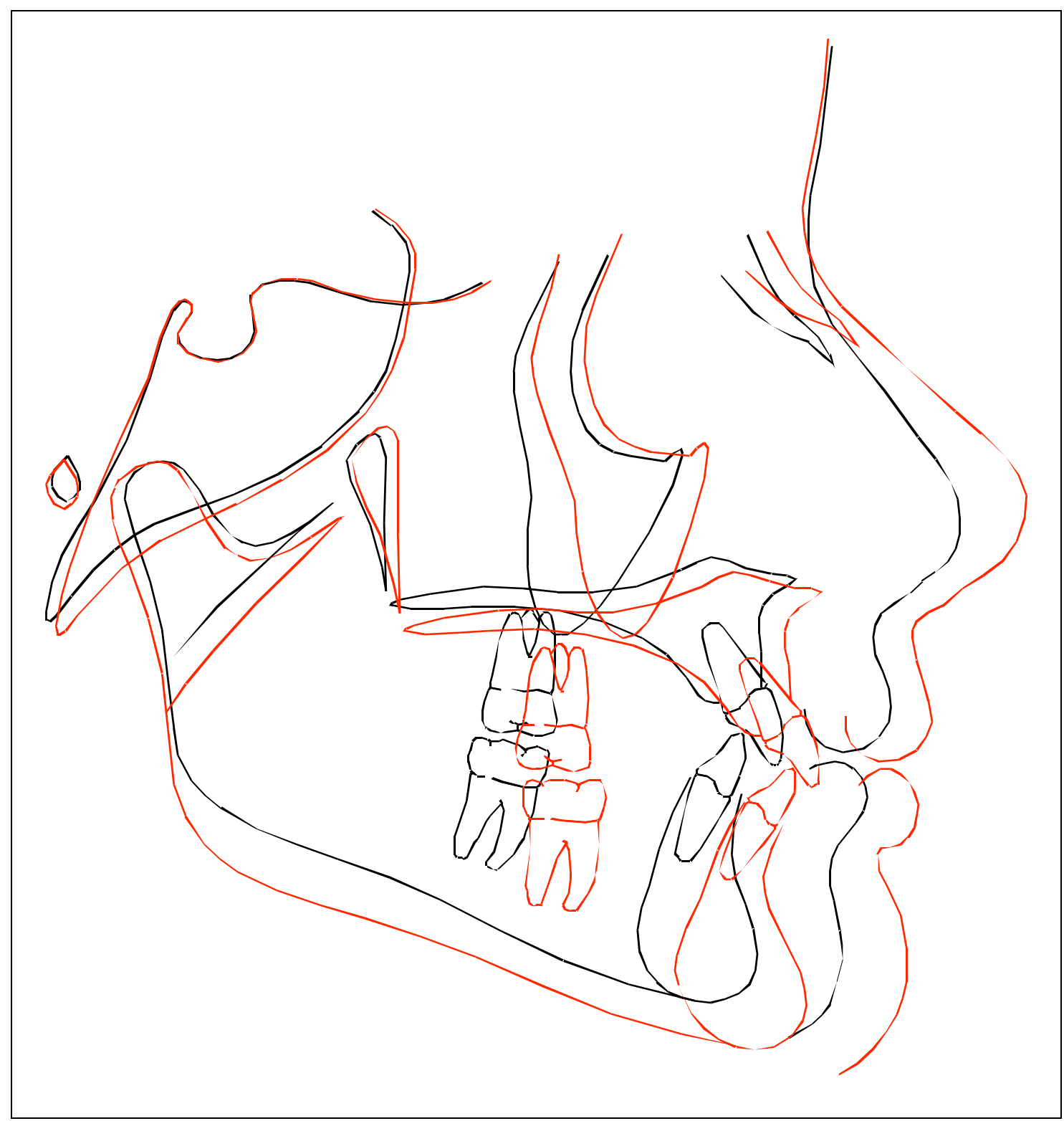

Figure A-12: Print of cephalometric tracing superimposition subject 12. 


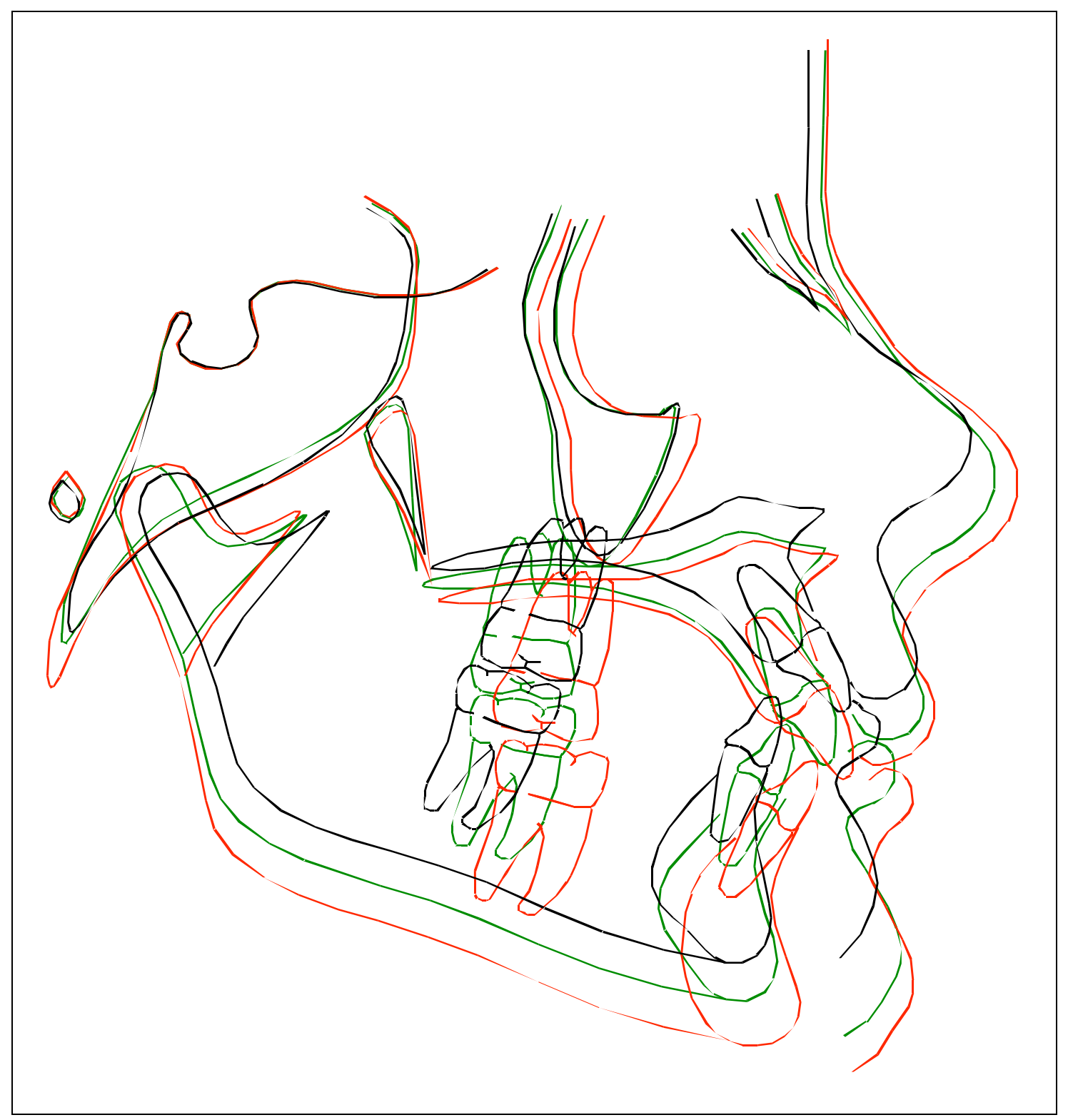

Figure A-13: Print of cephalometric tracing superimposition subject 13. 


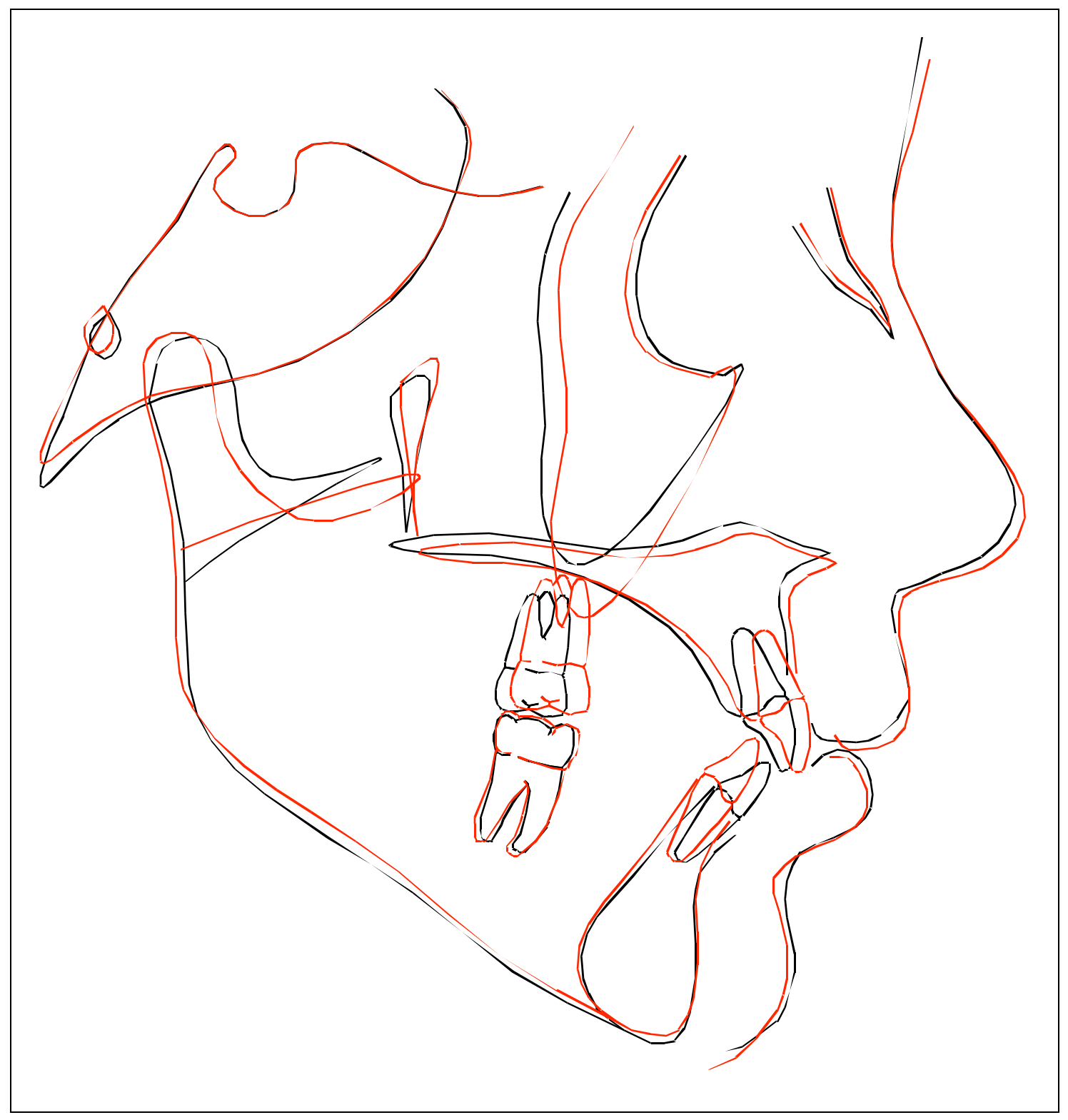

Figure A-14: Print of cephalometric tracing superimposition subject 14 . 


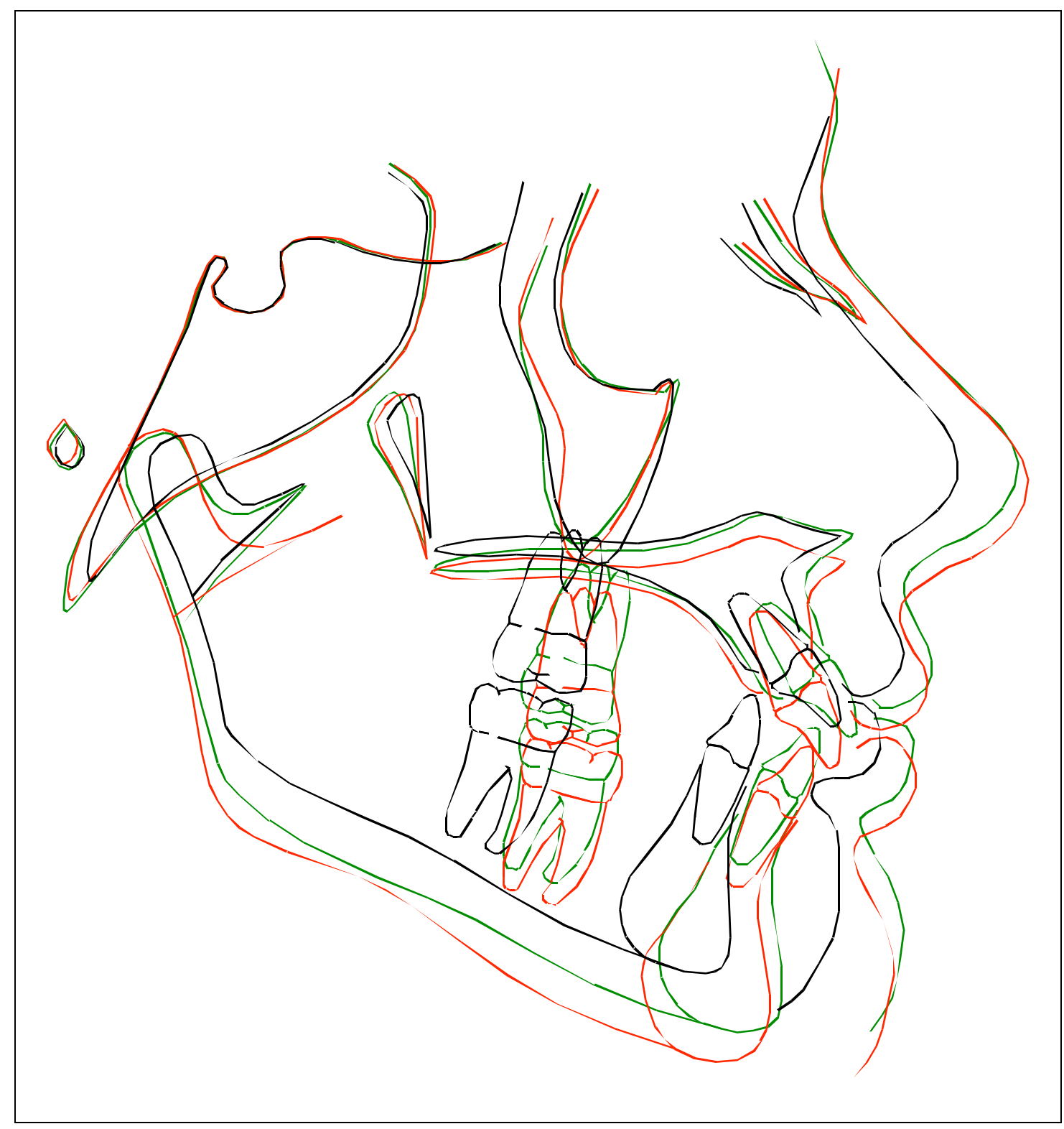

Figure A-15: Print of cephalometric tracing superimposition subject 15. 


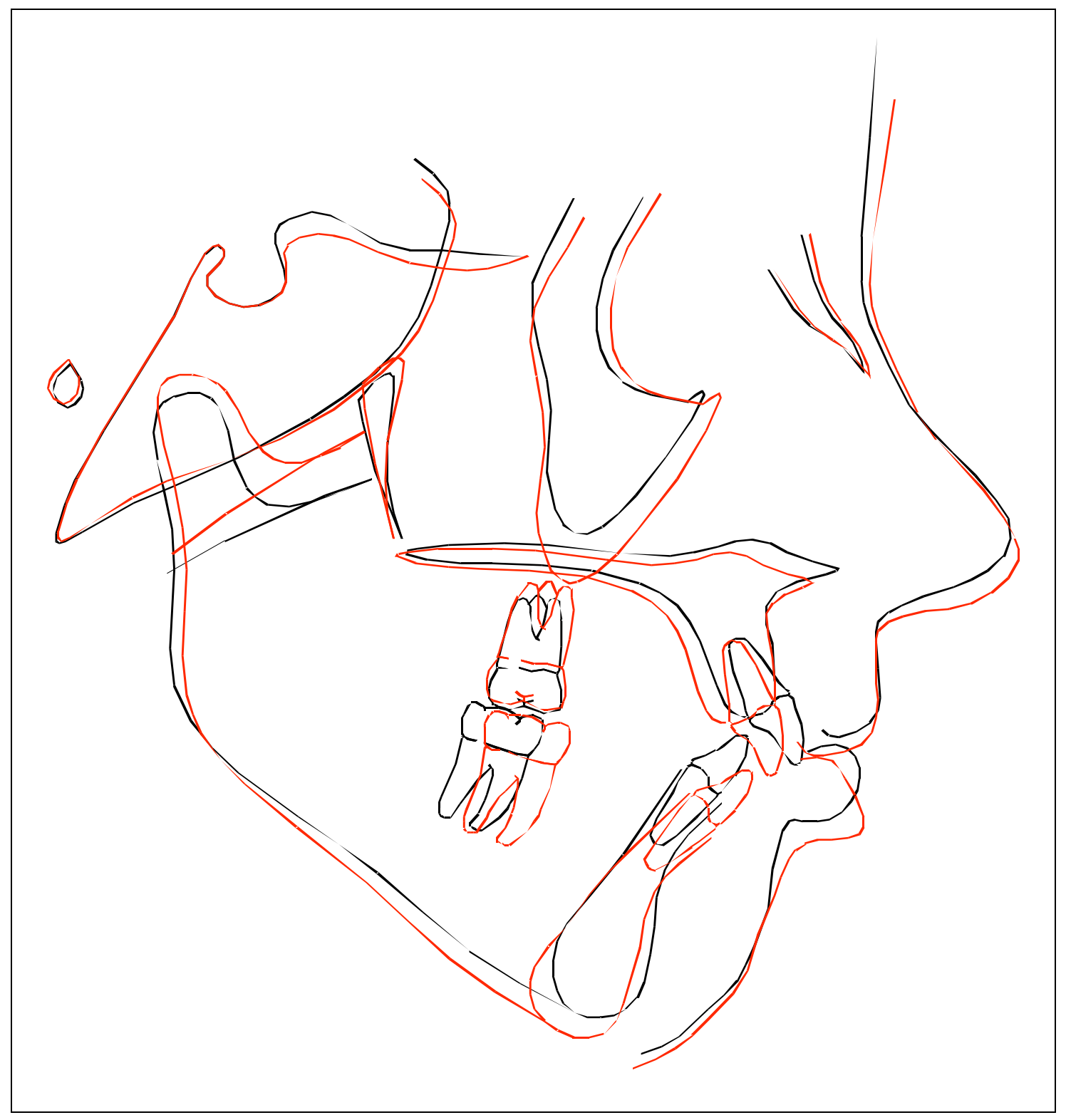

Figure A-16: Print of cephalometric tracing superimposition subject 16. 


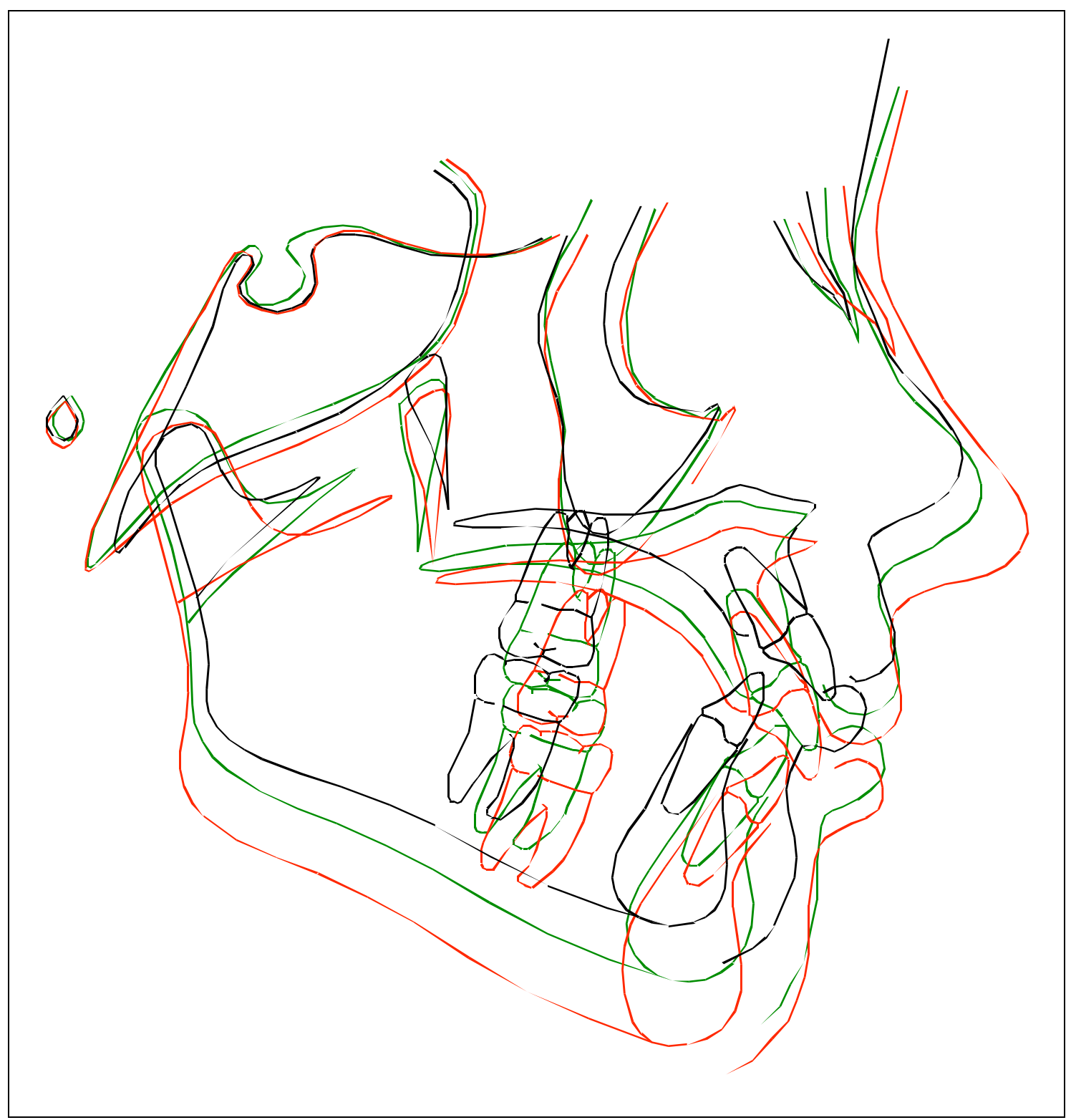

Figure A-17: Print of cephalometric tracing superimposition subject 17. 


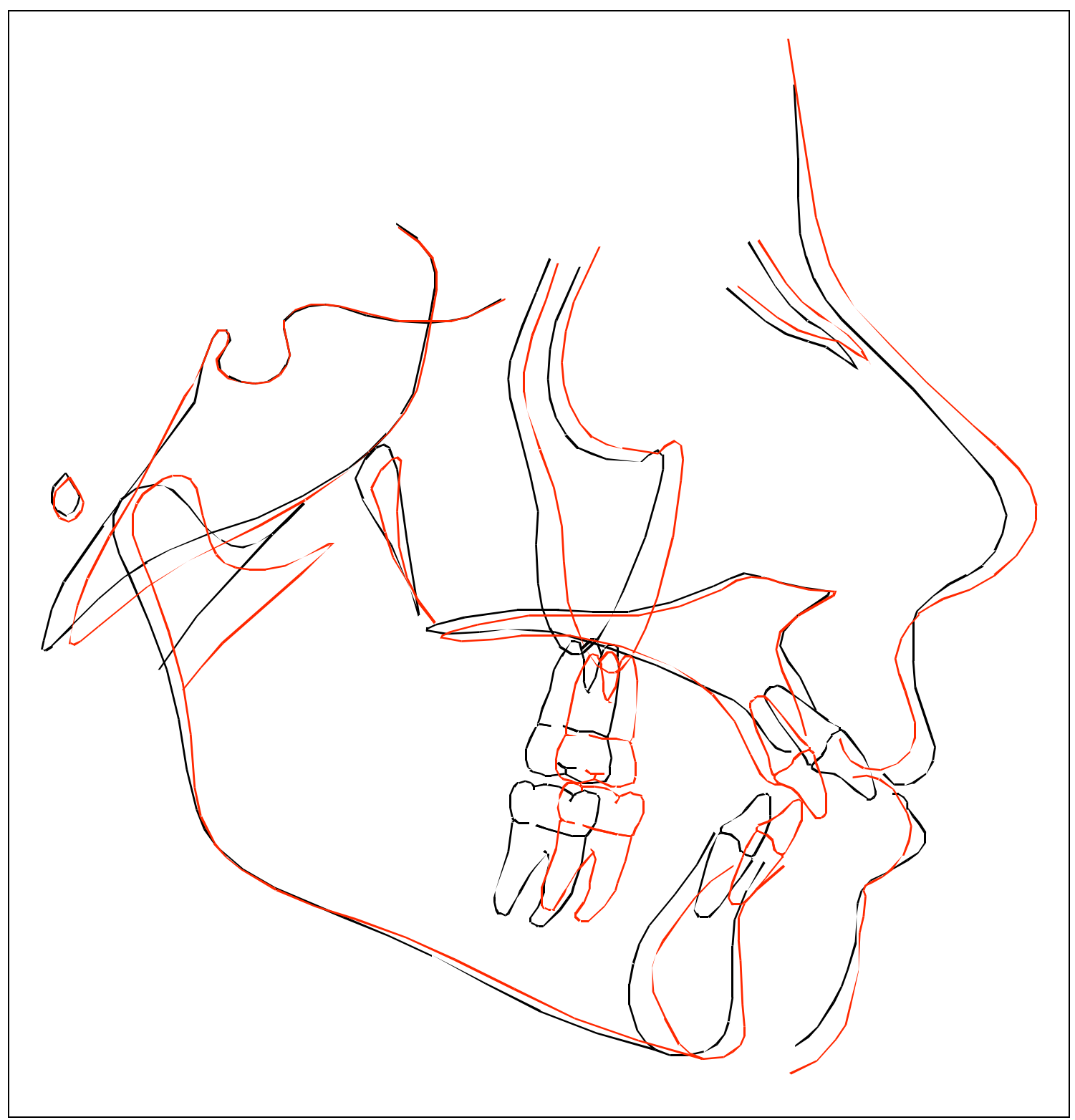

Figure A-18: Print of cephalometric tracing superimposition subject 18. 


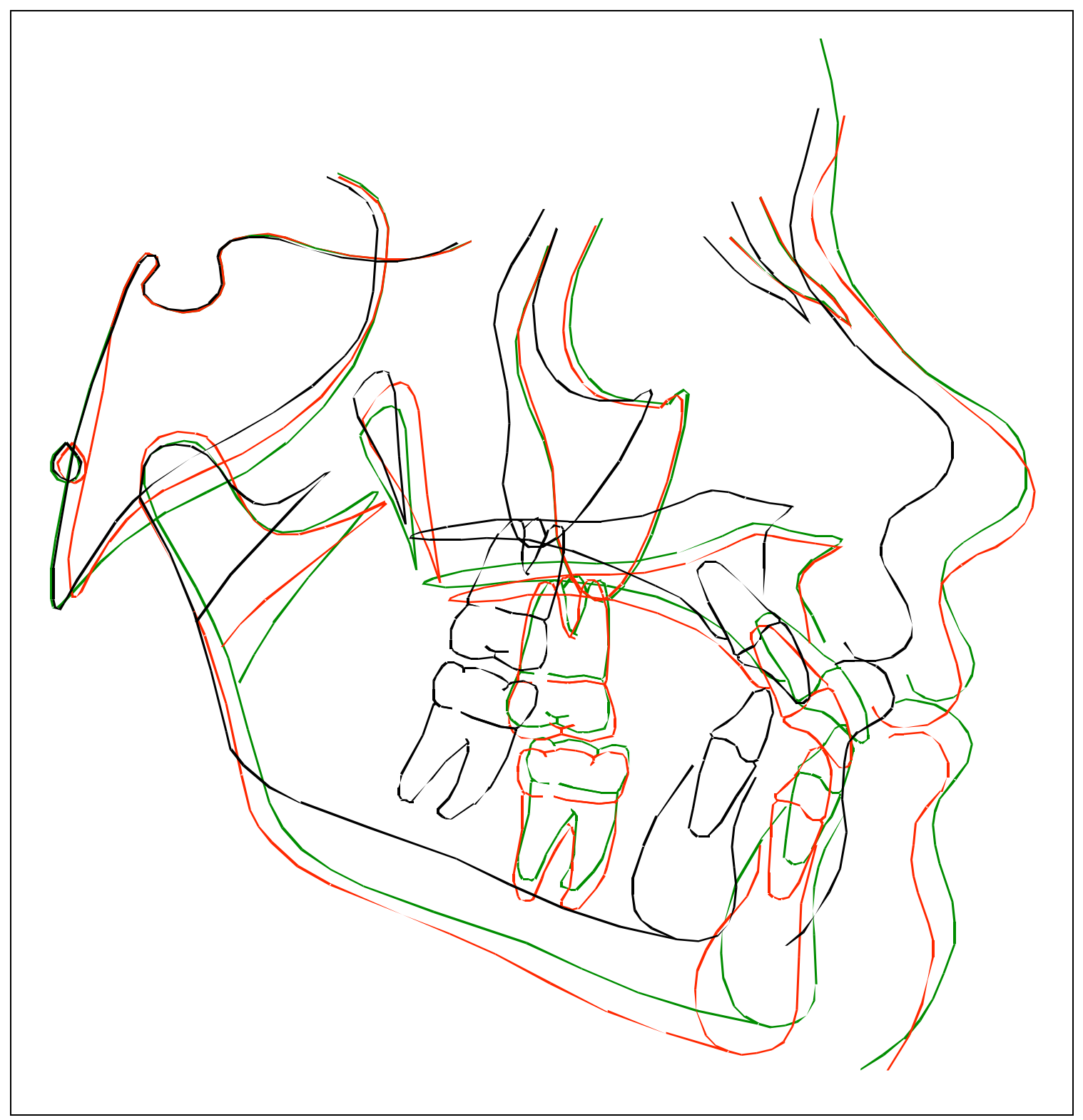

Figure A-19: Print of cephalometric tracing superimposition subject 19. 


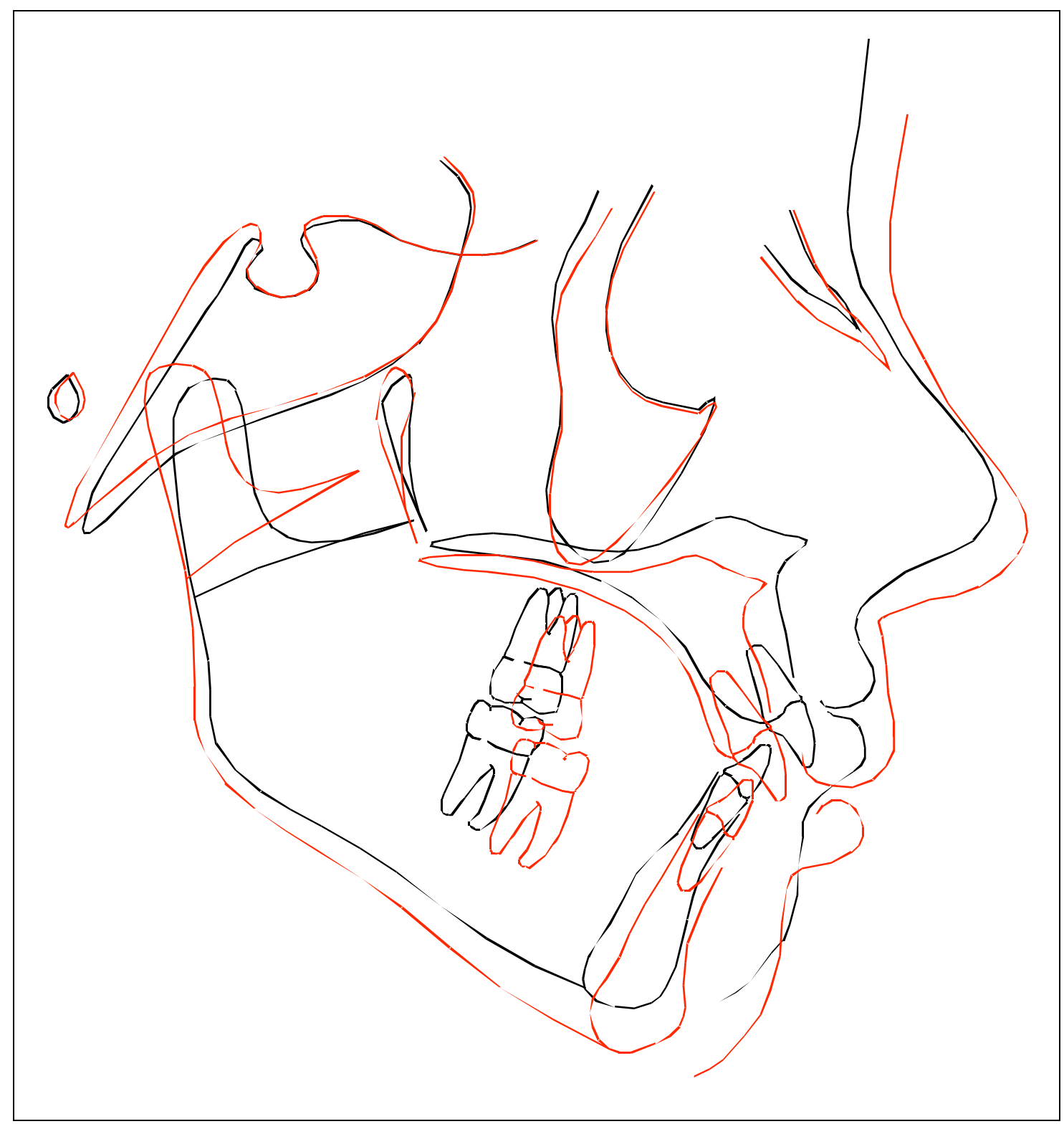

Figure A-20: Print of cephalometric tracing superimposition subject 20. 


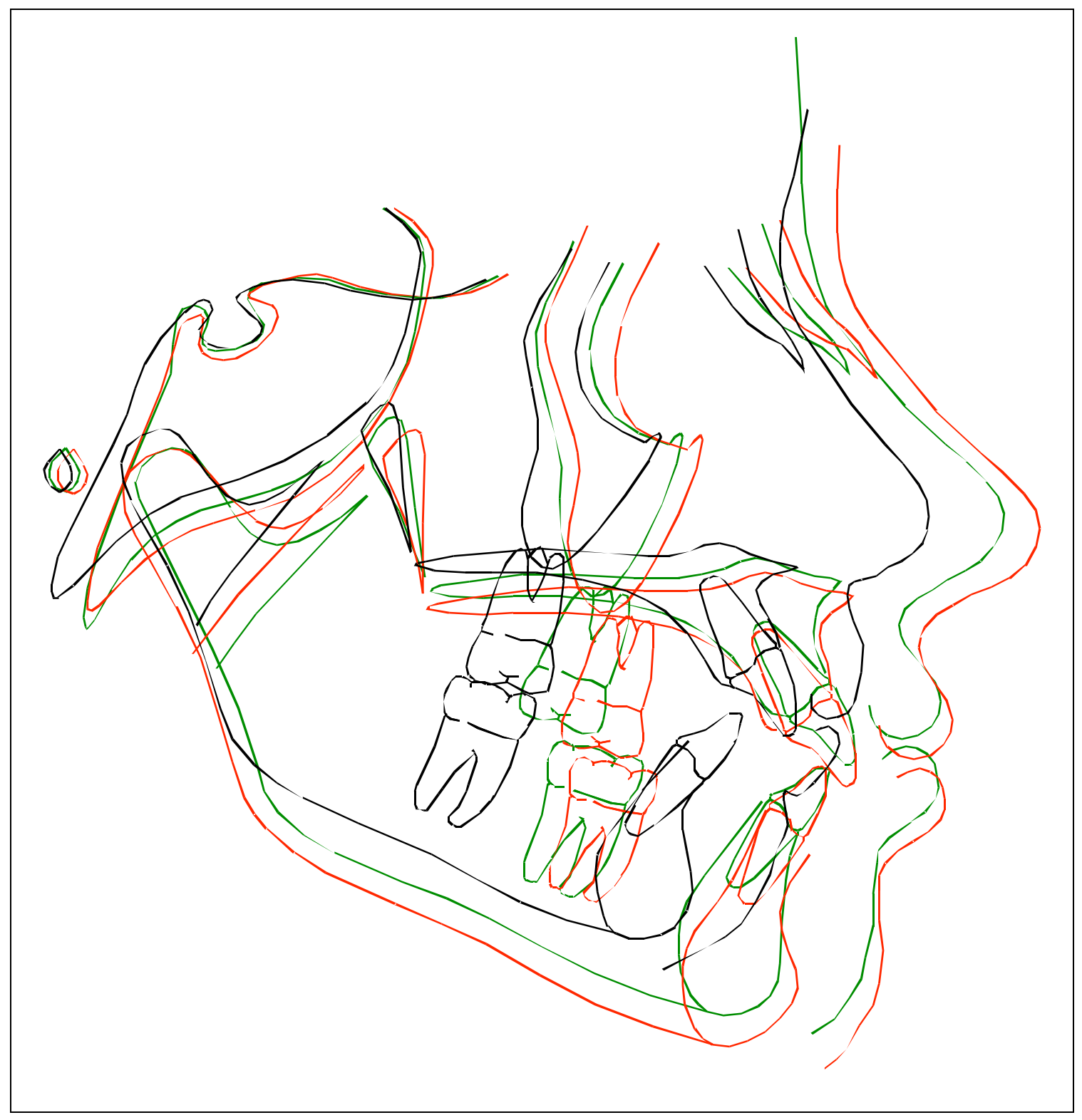

Figure A-21: Print of cephalometric tracing superimposition subject 21. 


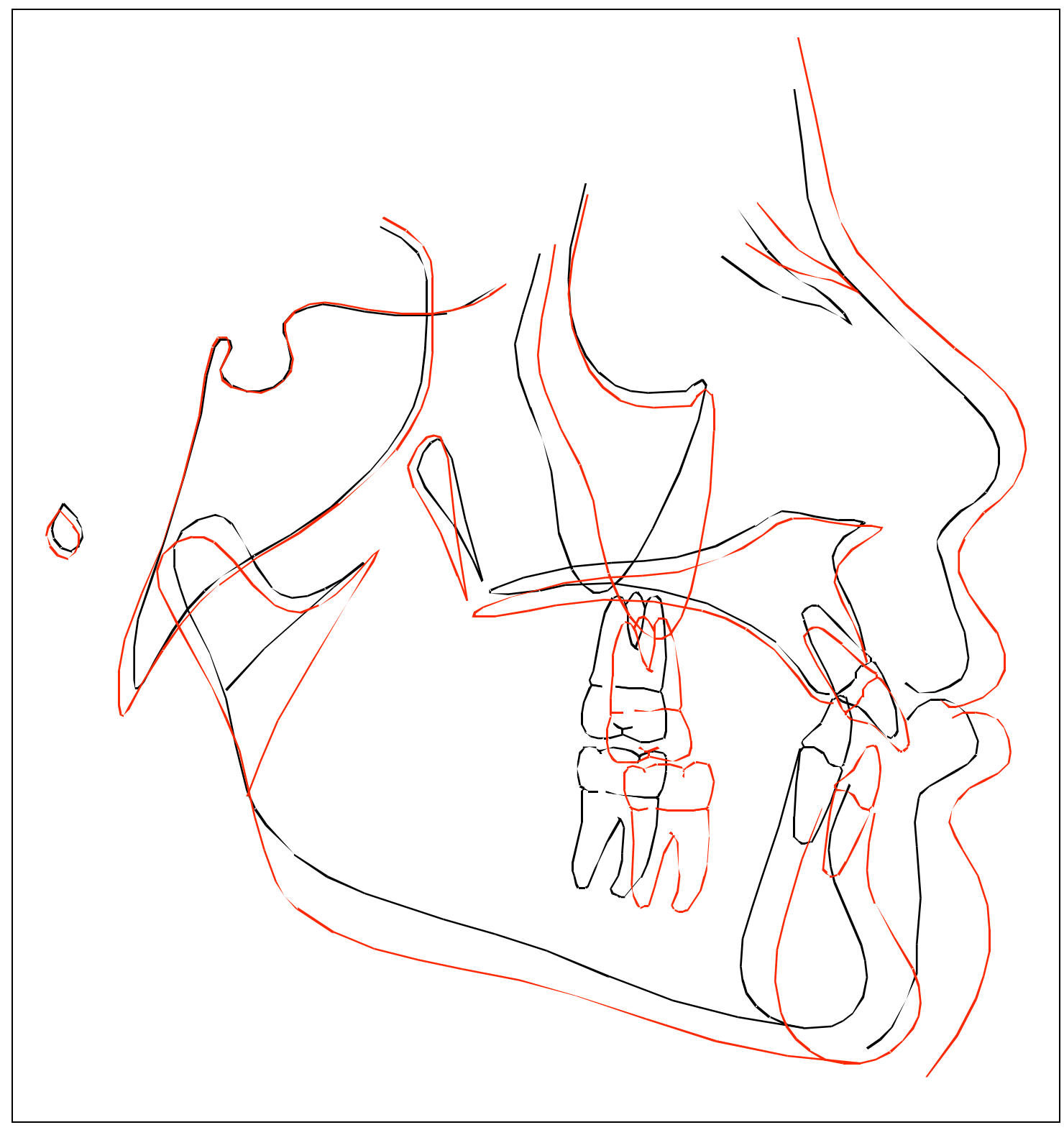

Figure A-22: Print of cephalometric tracing superimposition subject 22. 


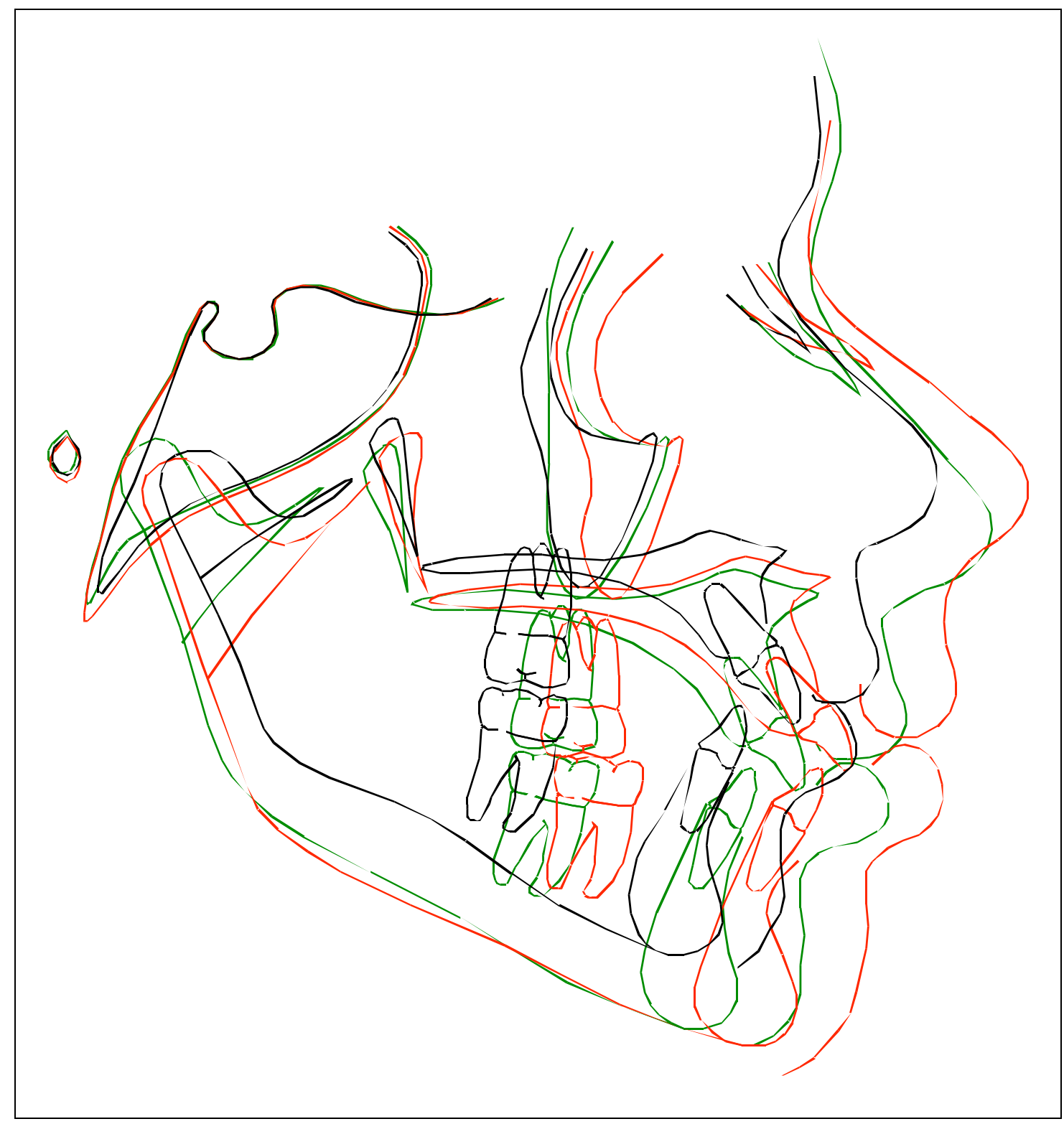

Figure A-23: Print of cephalometric tracing superimposition subject 23. 


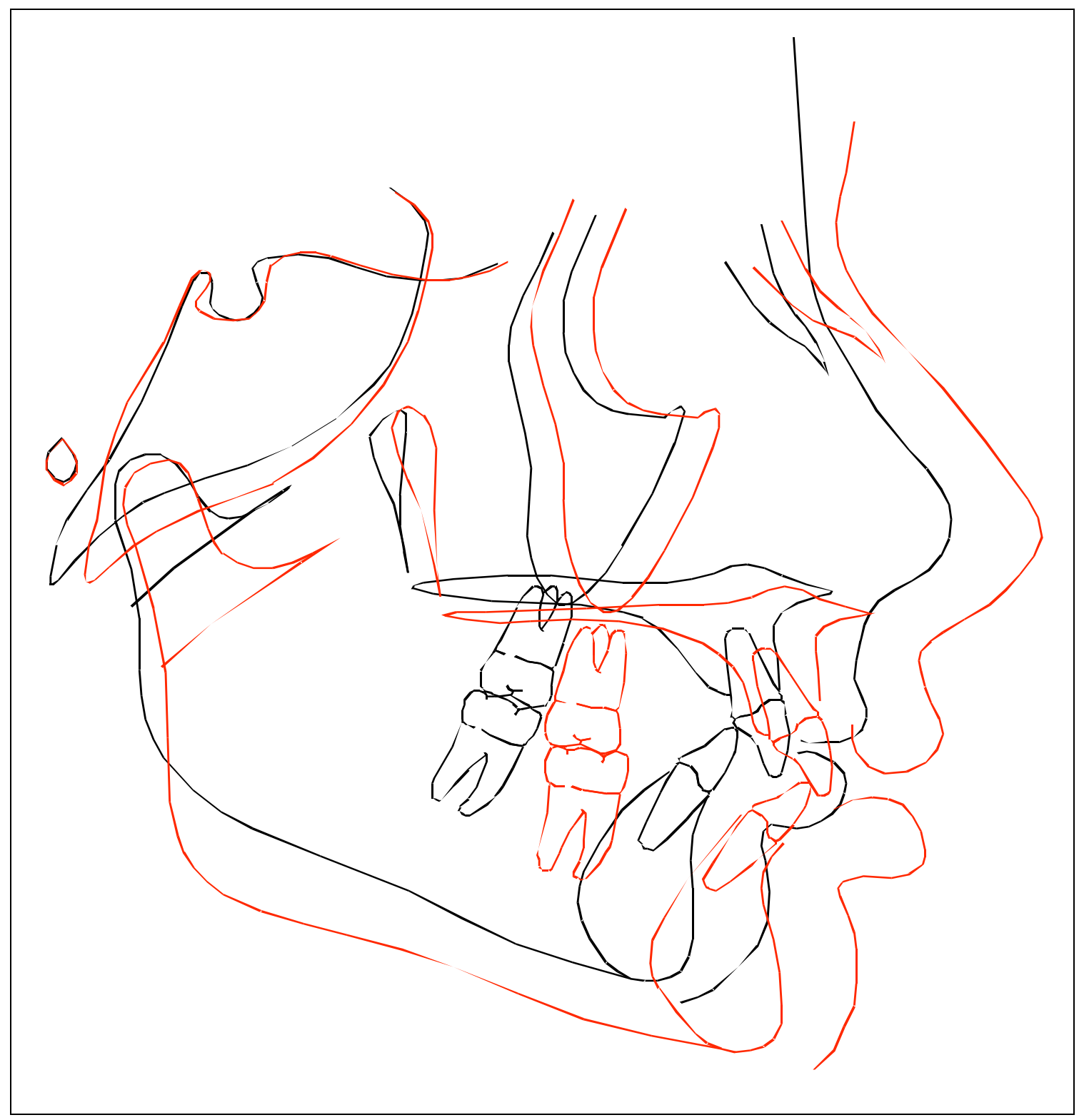

Figure A-24: Print of cephalometric tracing superimposition subject 24. 


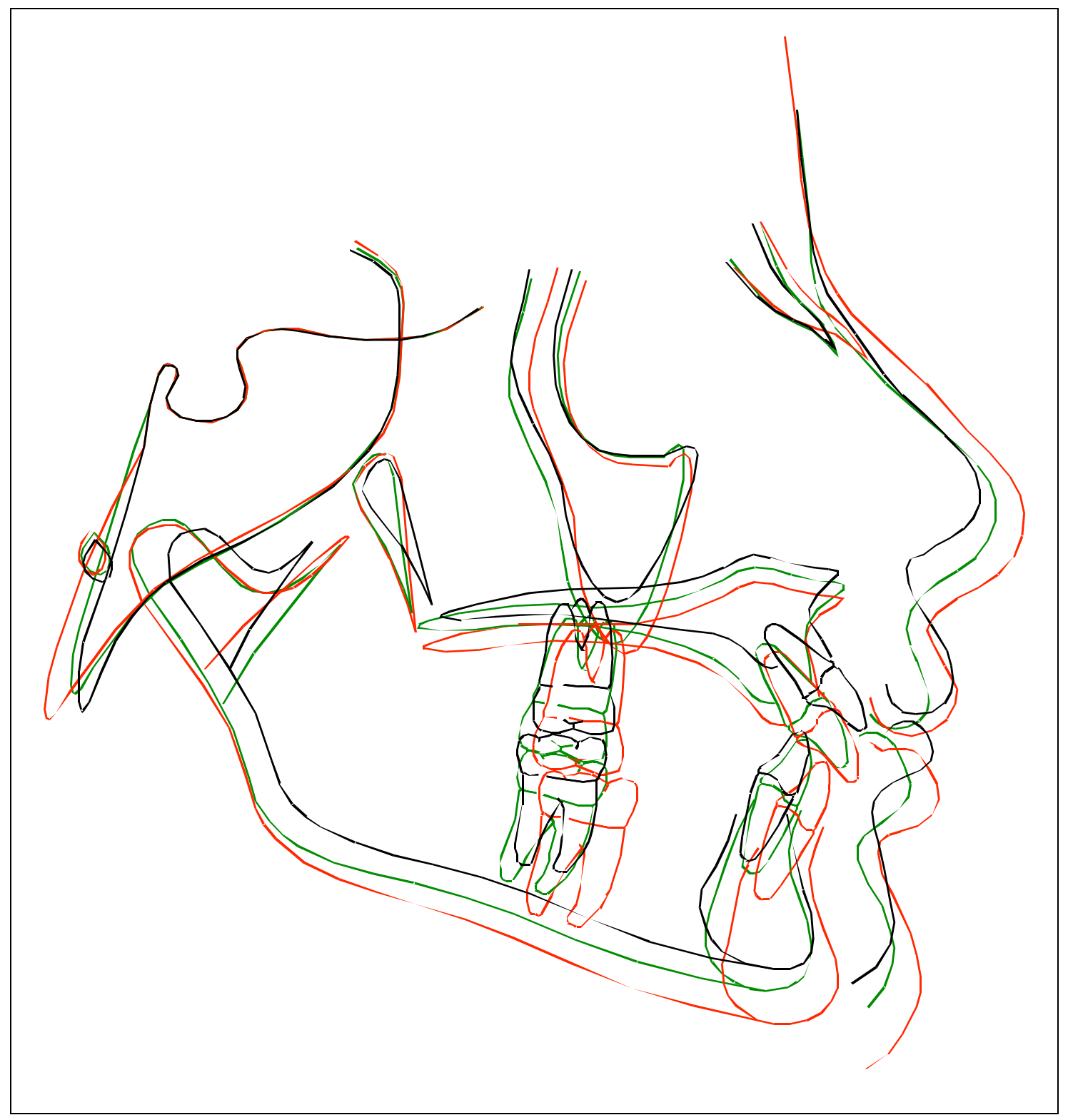

Figure A-25: Print of cephalometric tracing superimposition subject 25. 


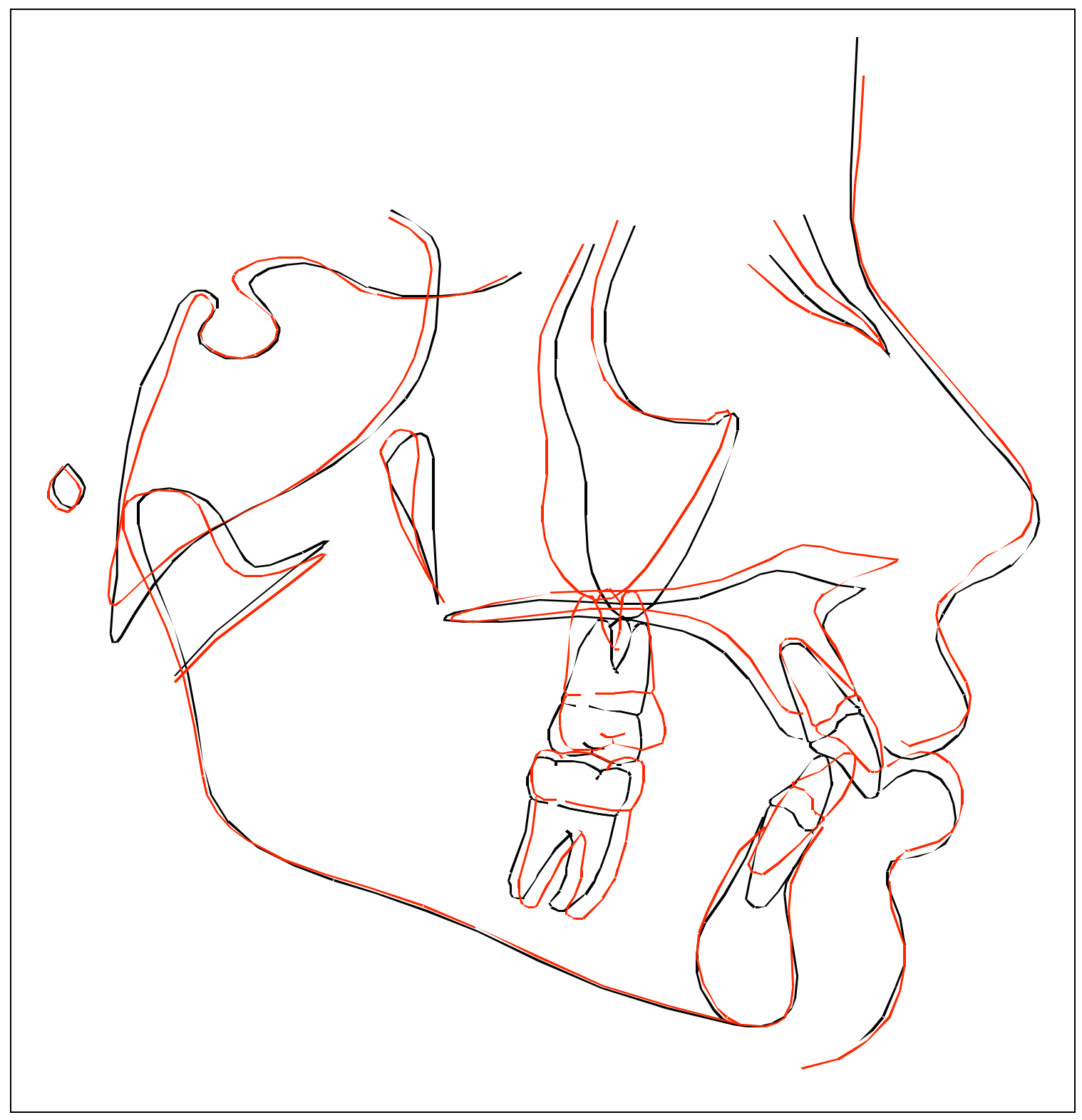

Figure A-26: Print of cephalometric tracing superimposition subject 26. 


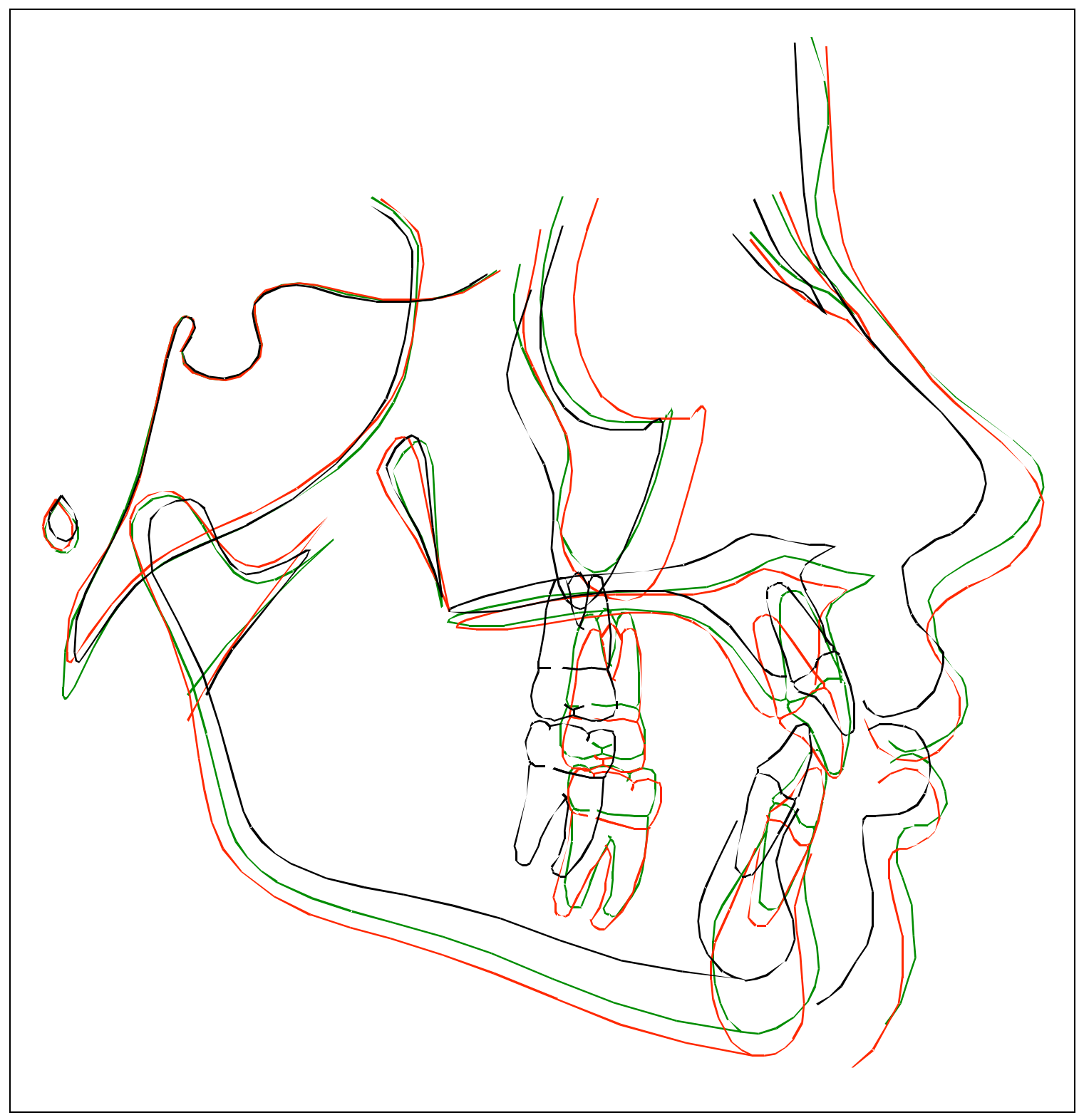

Figure A-27: Print of cephalometric tracing superimposition subject 27. 


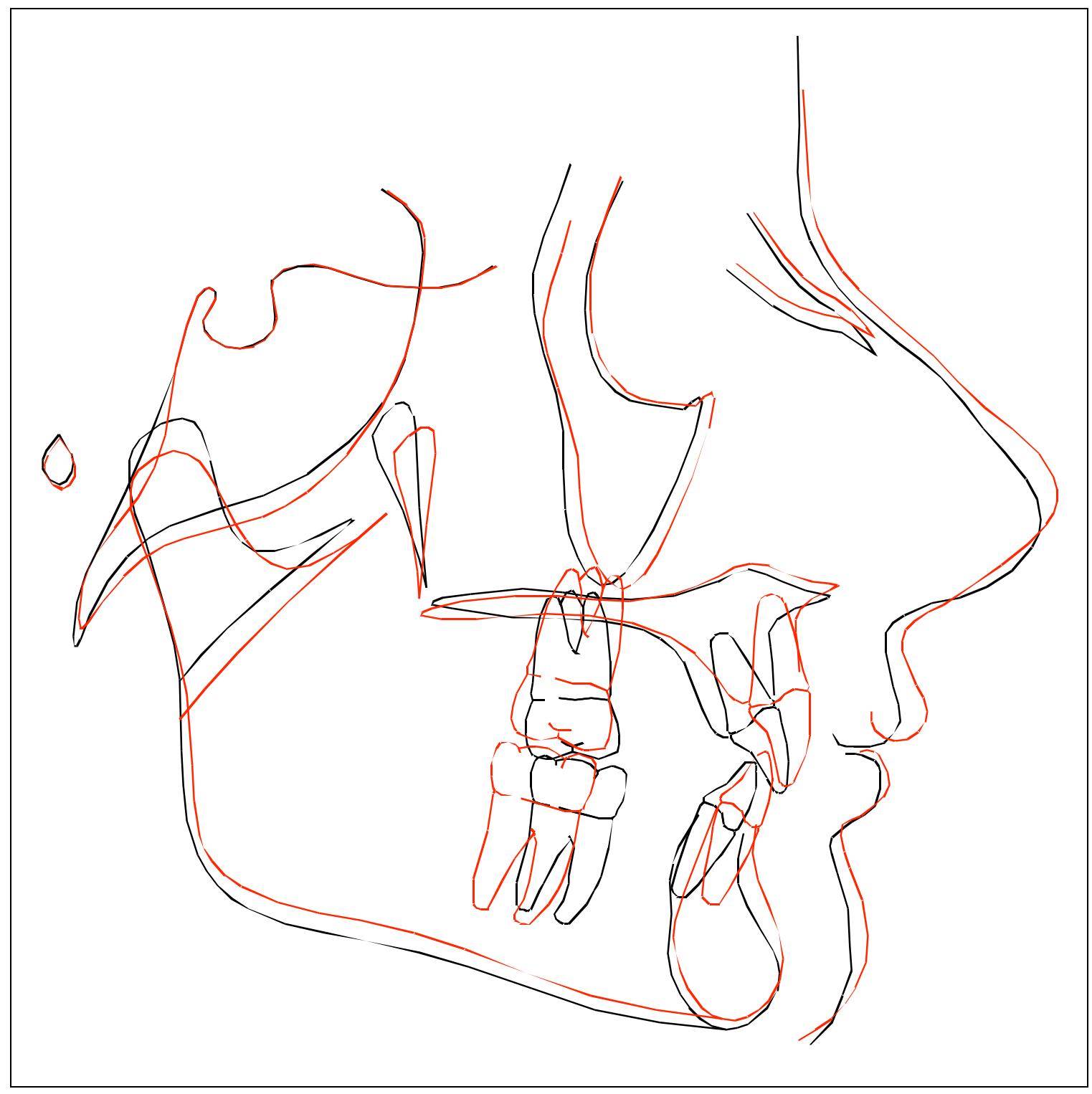

Figure A-28: Print of cephalometric tracing superimposition subject 28. 


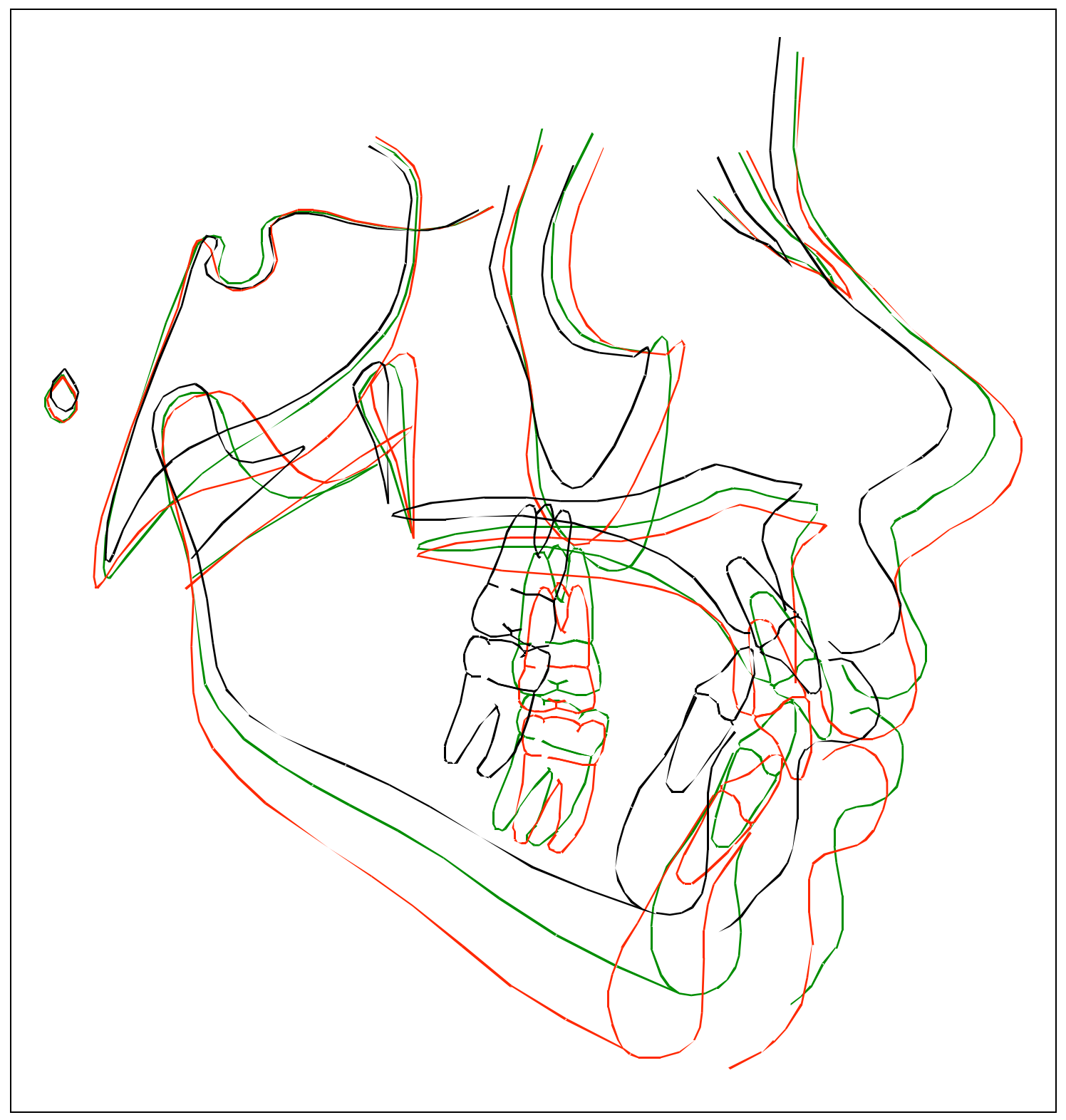

Figure A-29: Print of cephalometric tracing superimposition subject 29. 


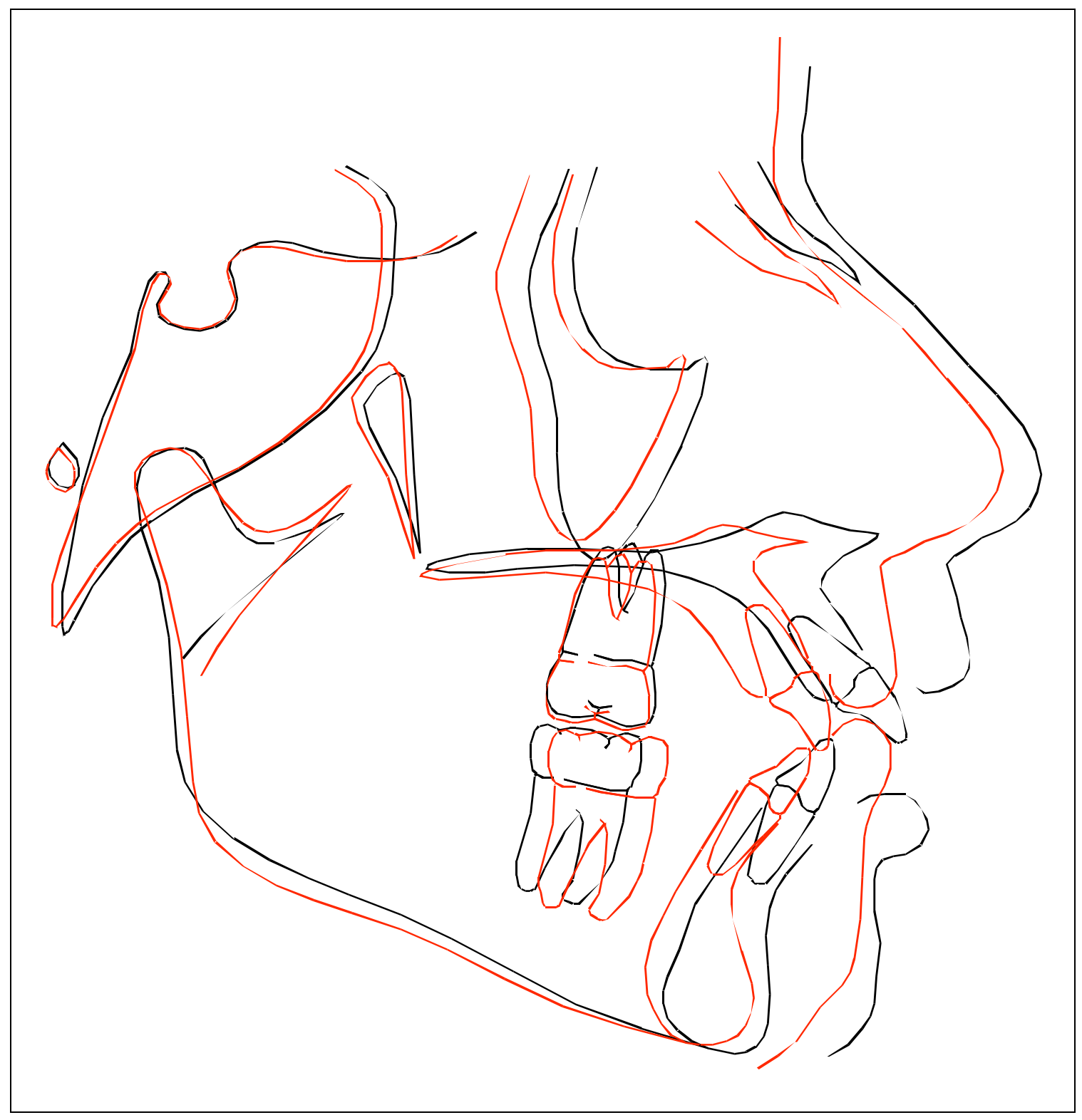

Figure A-30: Print of cephalometric tracing superimposition subject 30. 


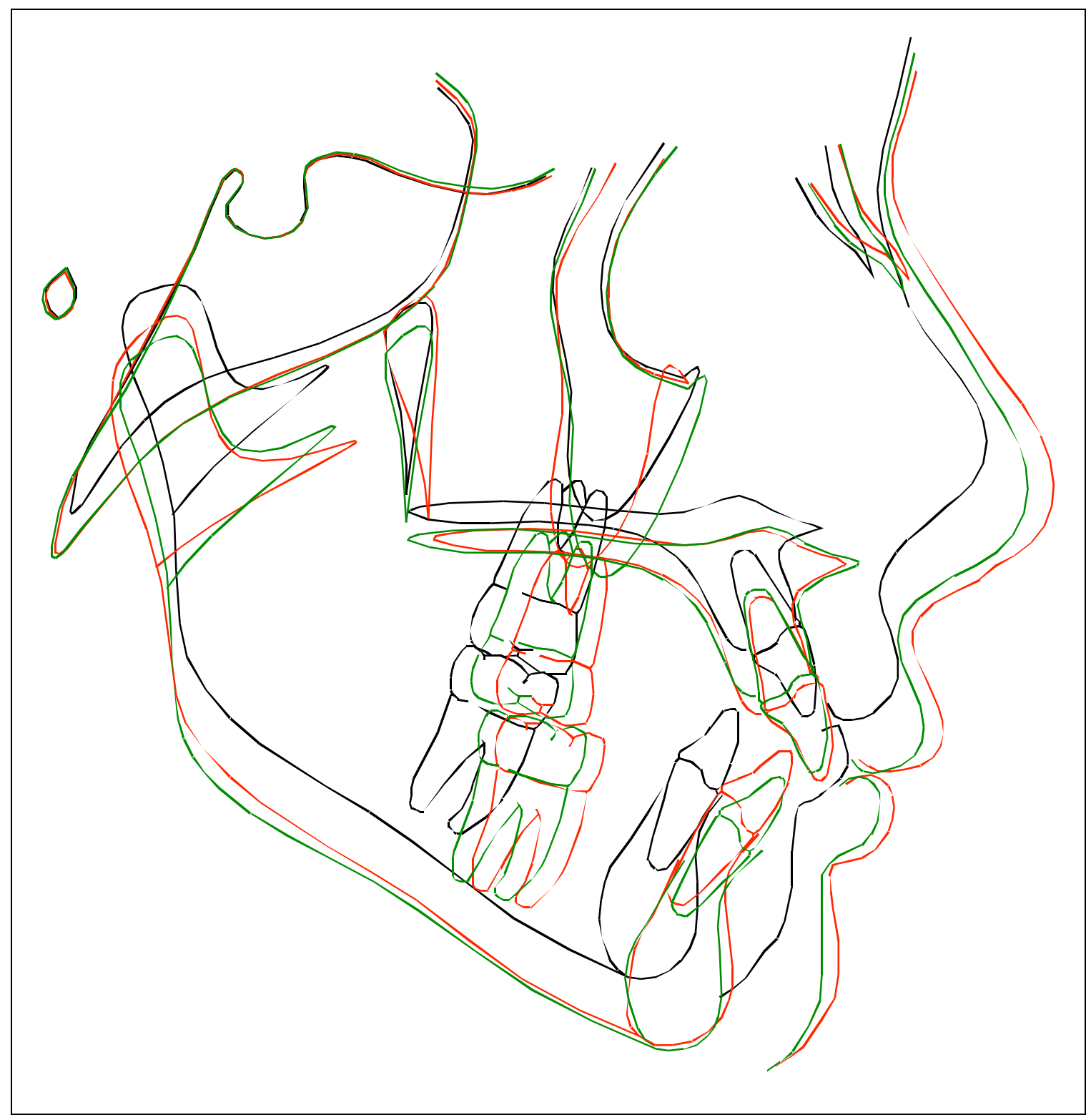

Figure A-31: Print of cephalometric tracing superimposition subject 31. 


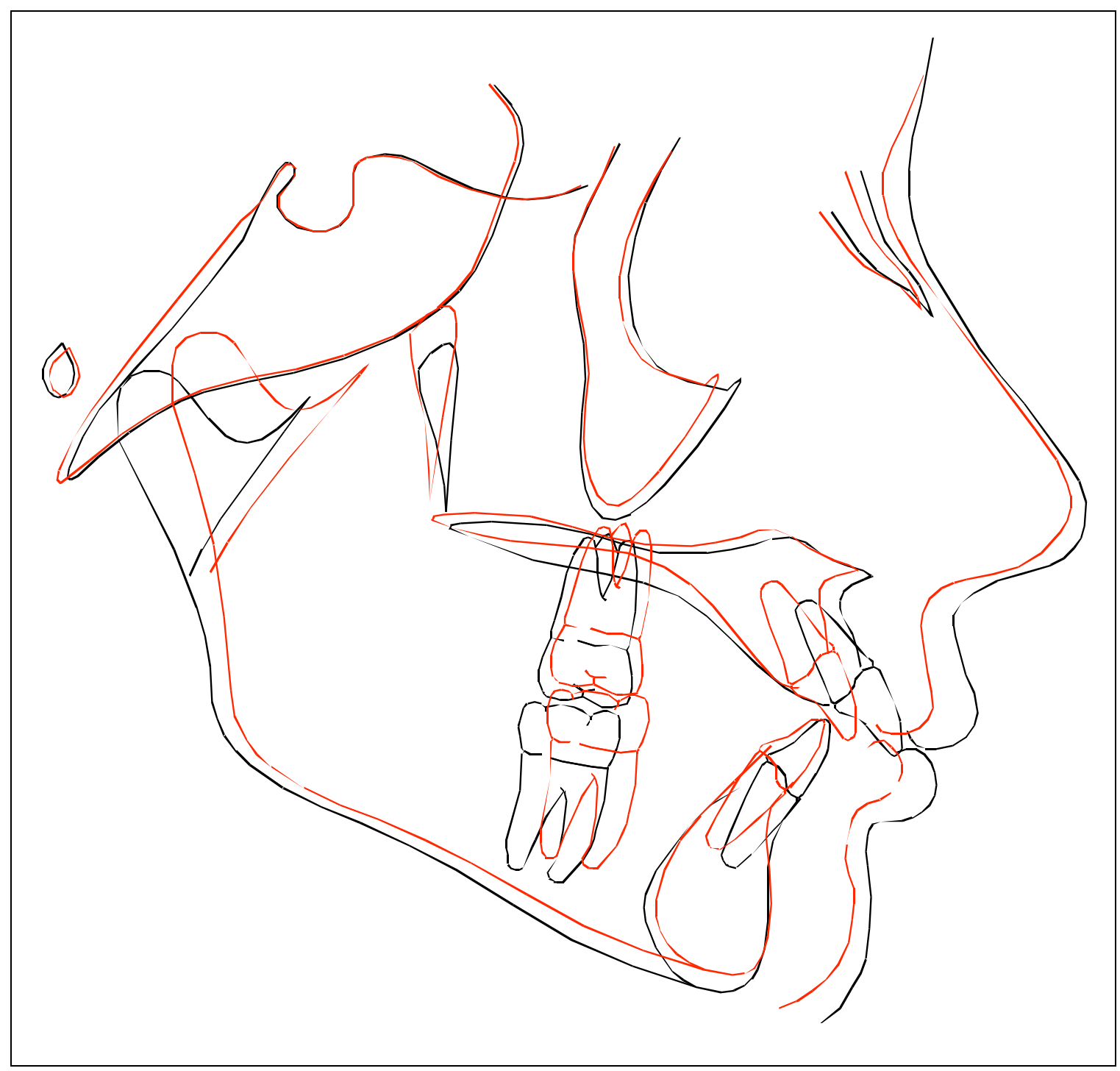

Figure A-32: Print of cephalometric tracing superimposition subject 32. 


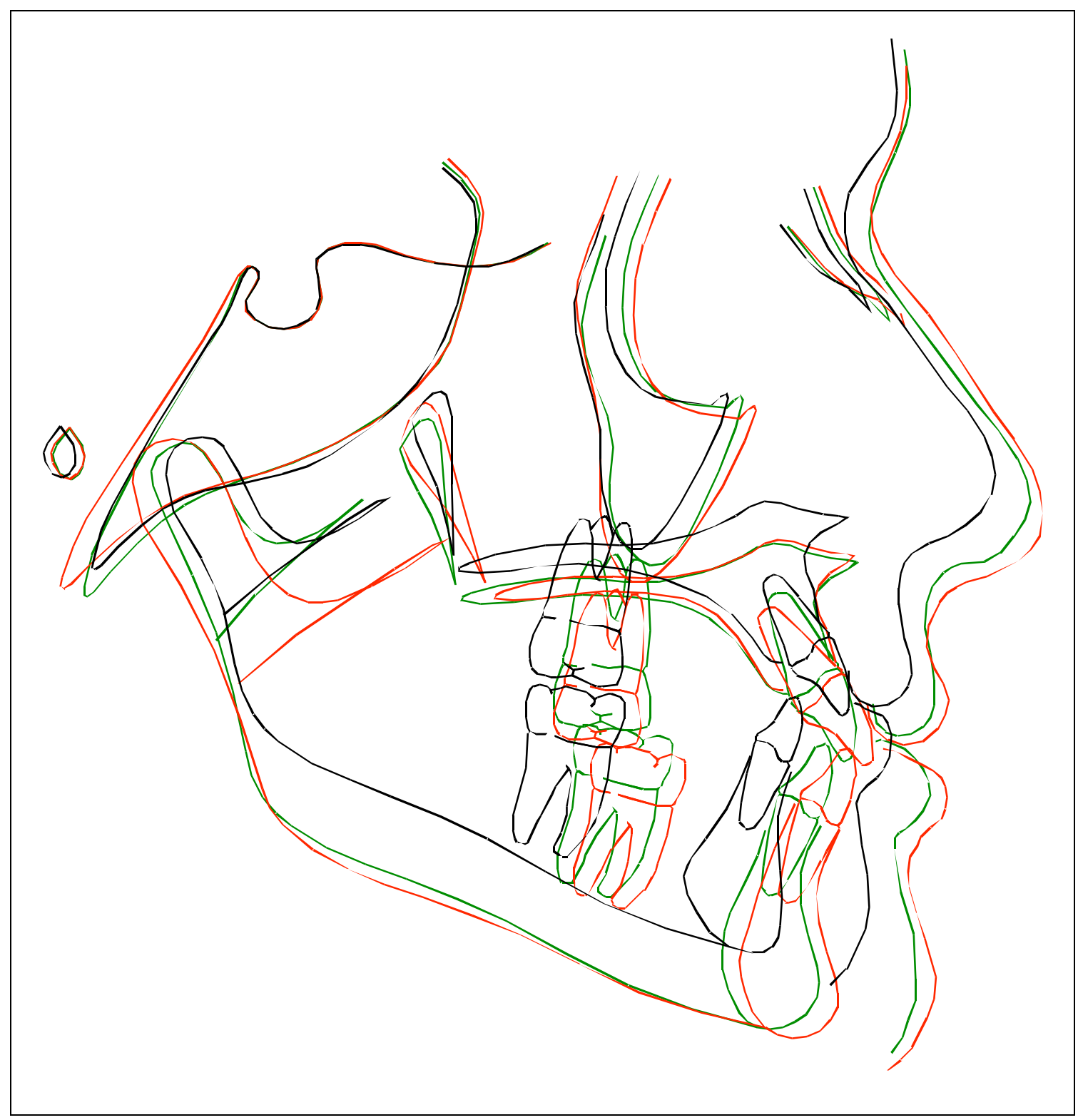

Figure A-33: Print of cephalometric tracing superimposition subject 33. 


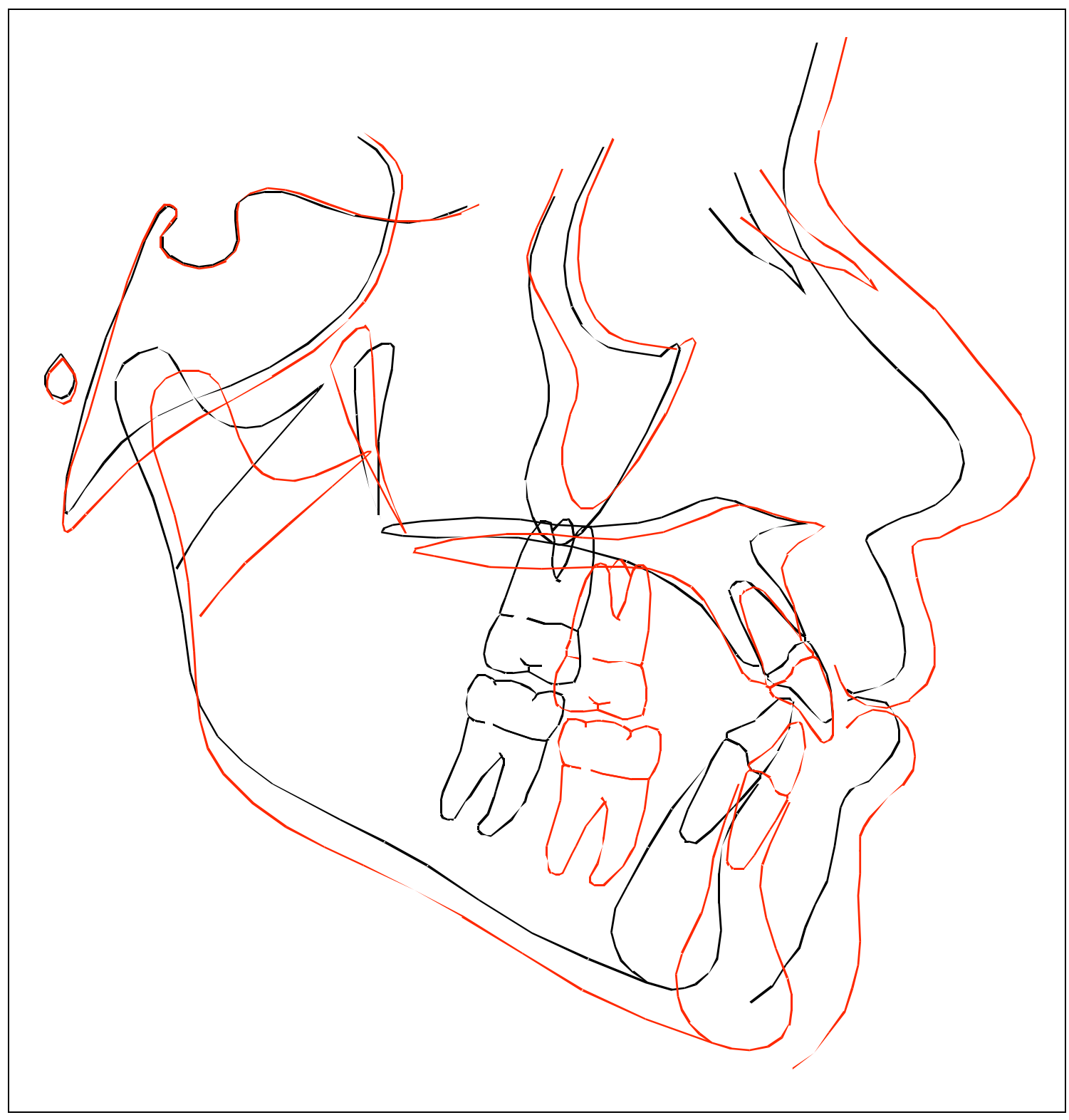

Figure A-34: Print of cephalometric tracing superimposition subject 34. 


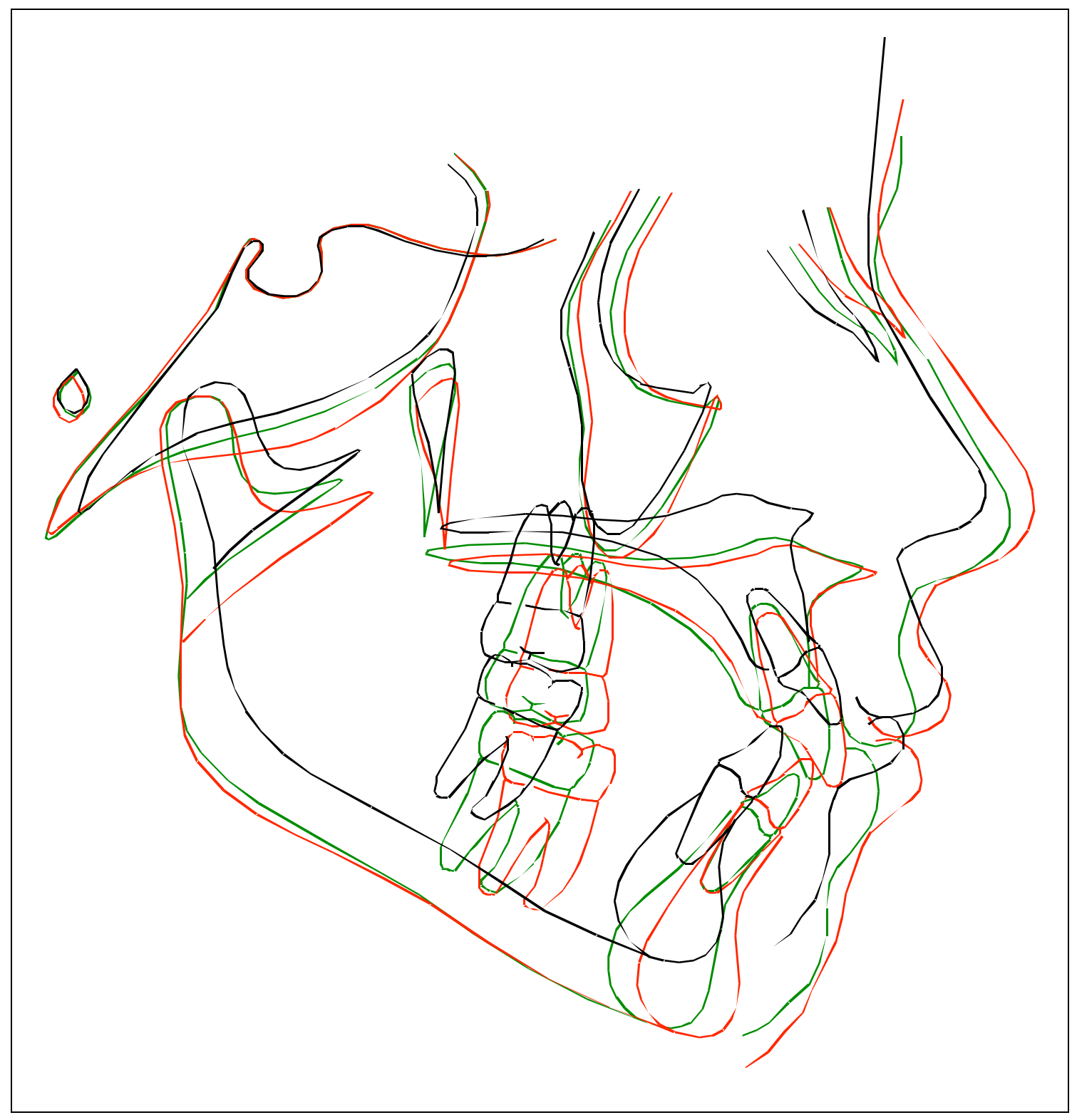

Figure A-35: Print of cephalometric tracing superimposition subject 35. 


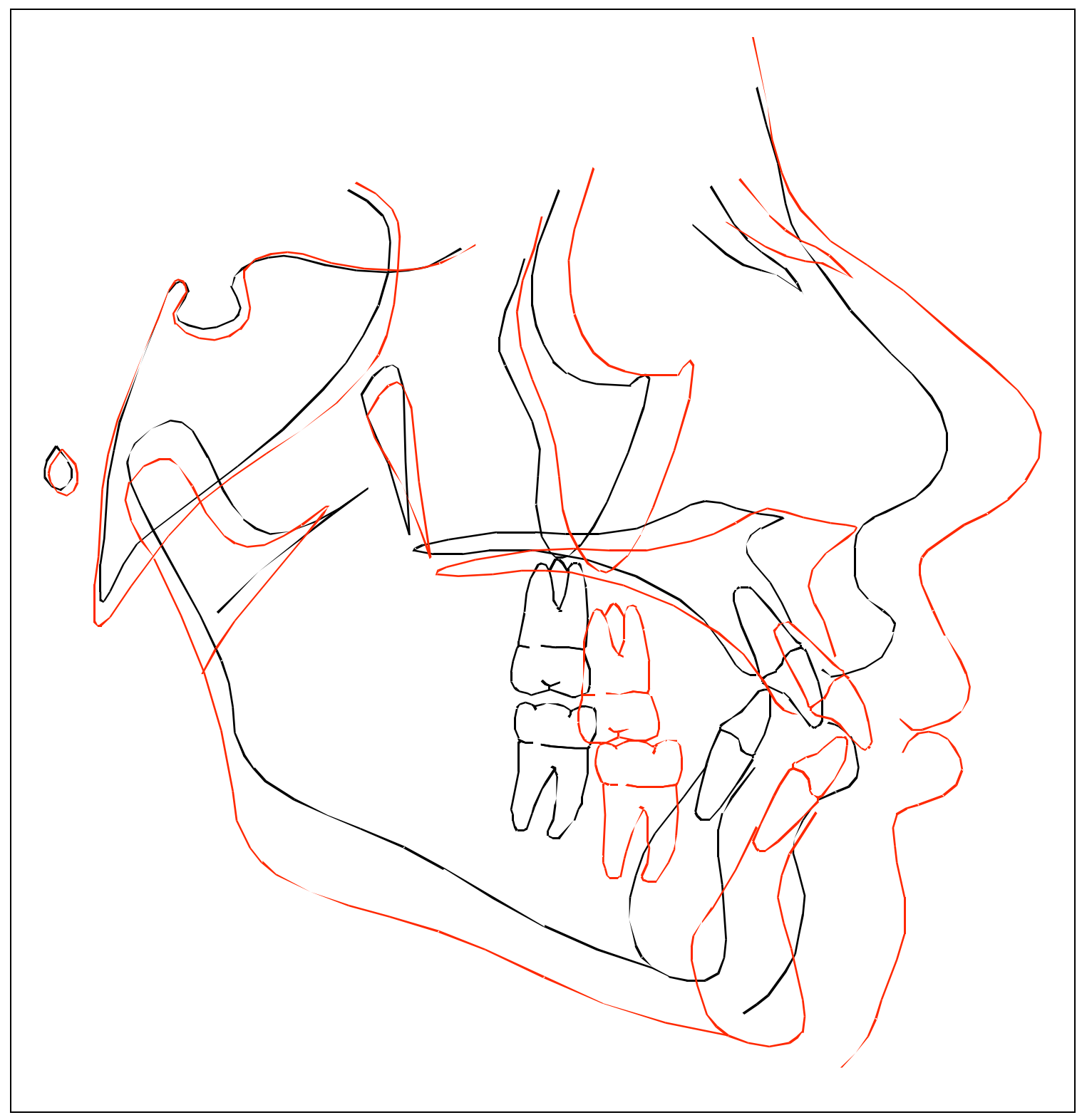

Figure A-36: Print of cephalometric tracing superimposition subject 36. 


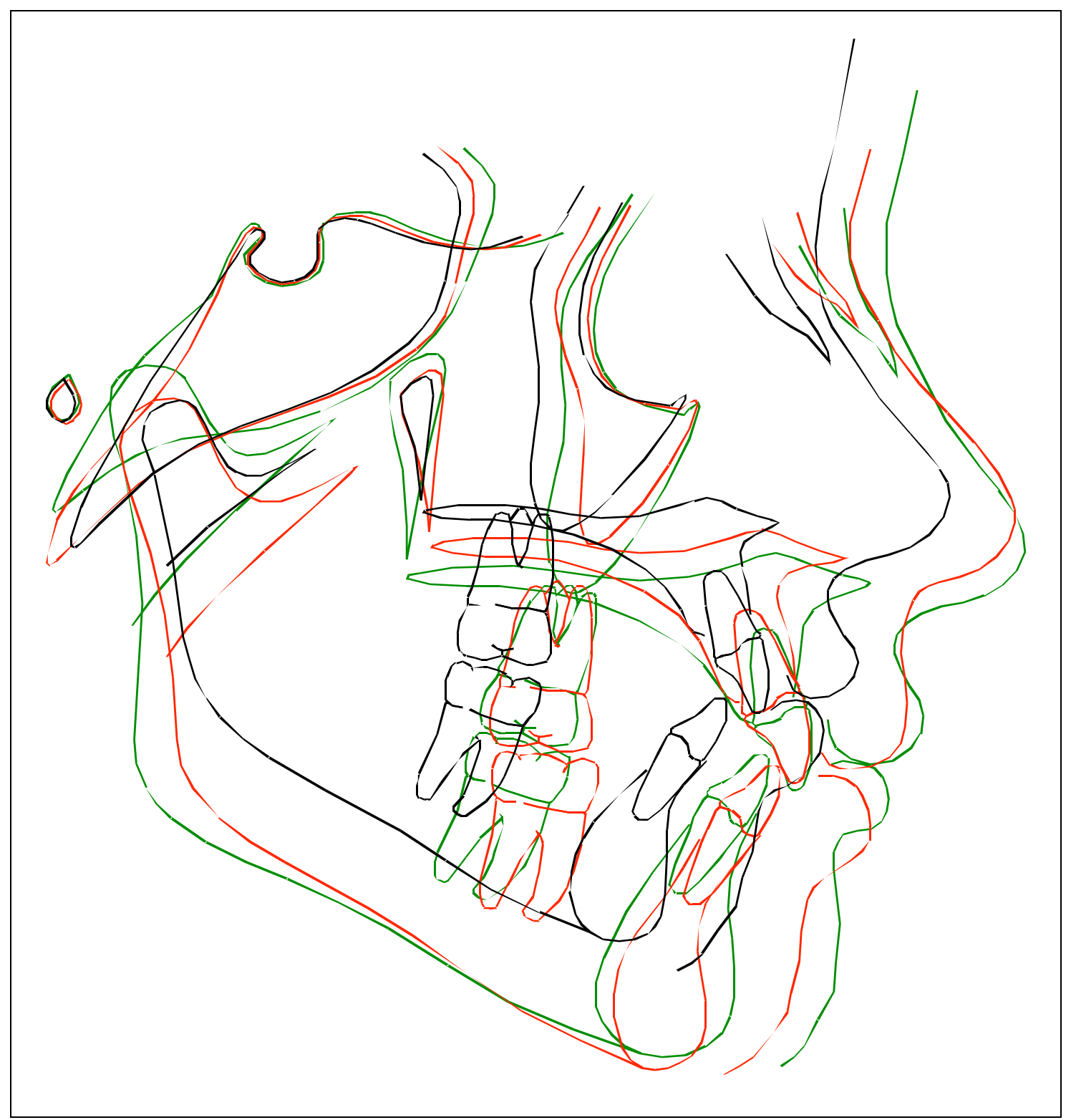

Figure A-37: Print of cephalometric tracing superimposition subject 37. 


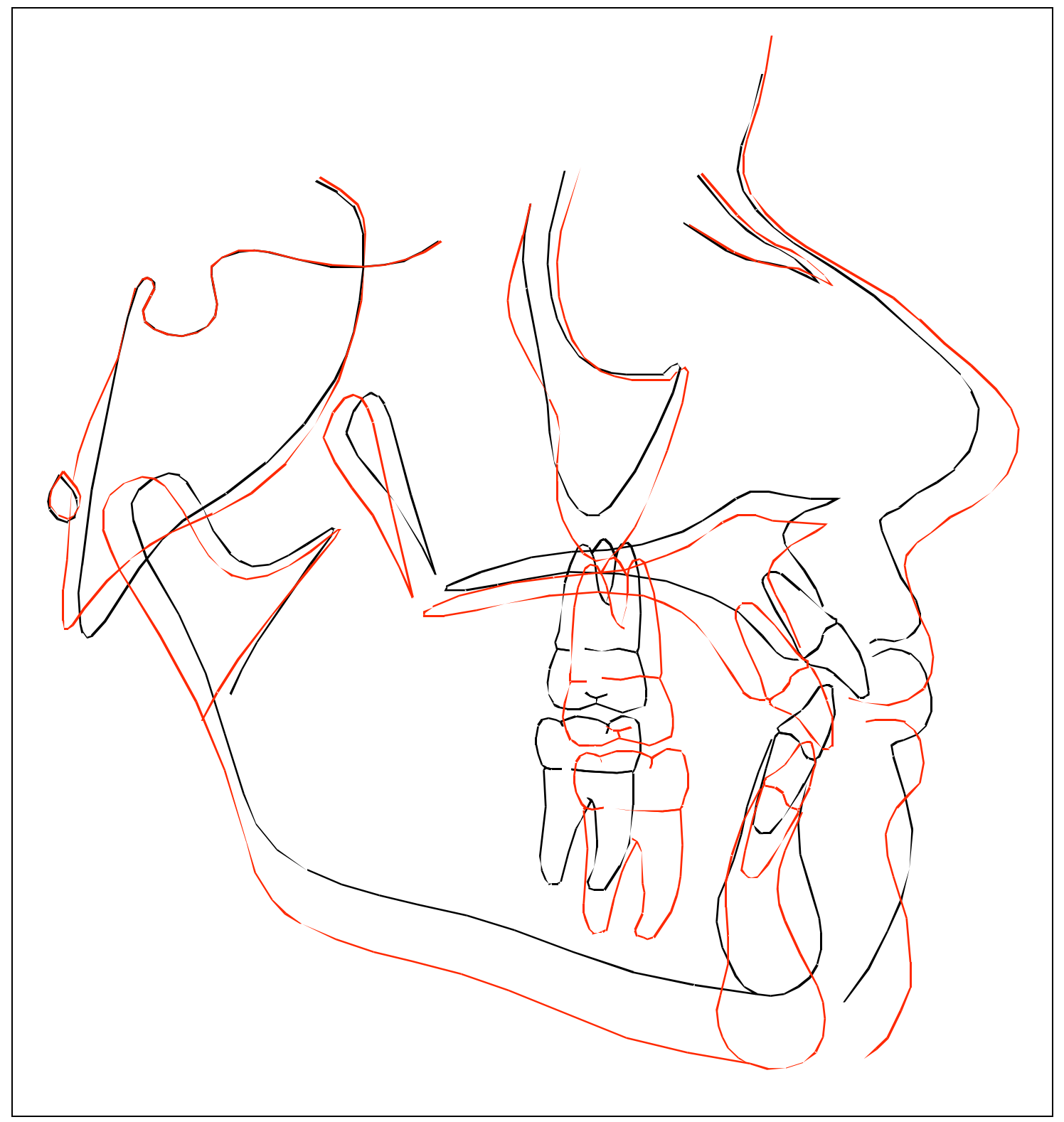

Figure A-38: Print of cephalometric tracing superimposition subject 38. 


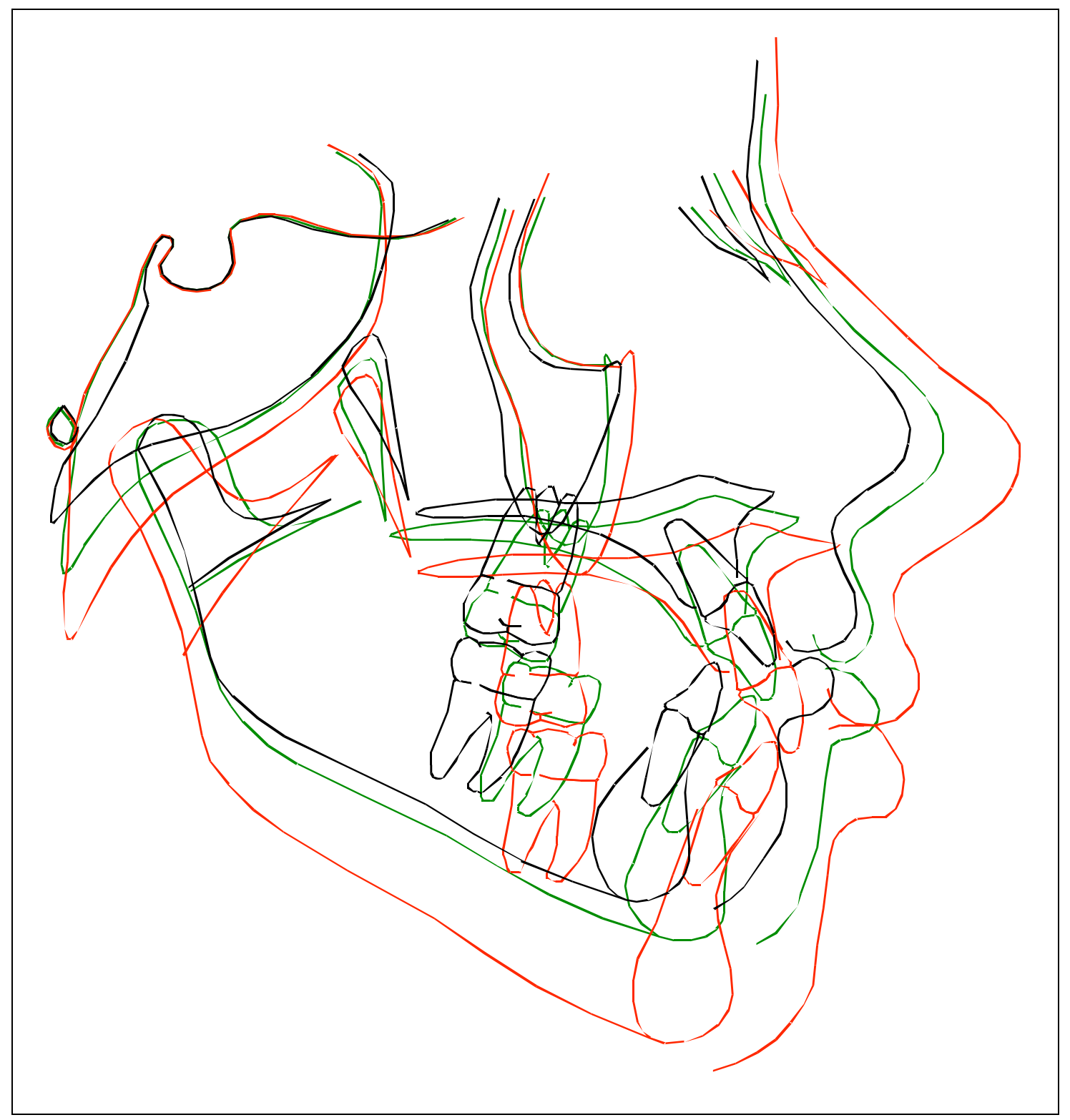

Figure A-39: Print of cephalometric tracing superimposition subject 39. 


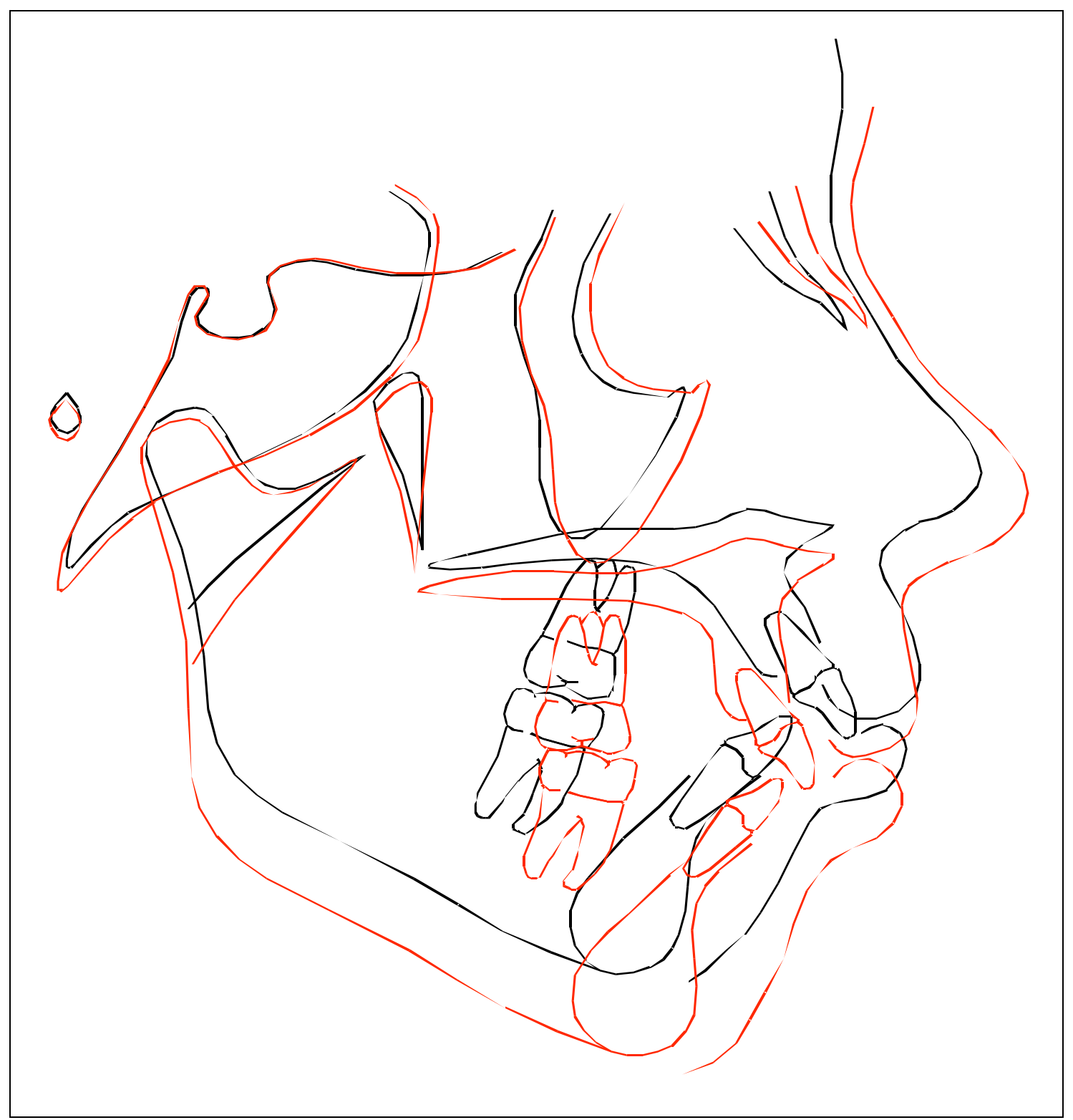

Figure A-40: Print of cephalometric tracing superimposition subject 40 . 


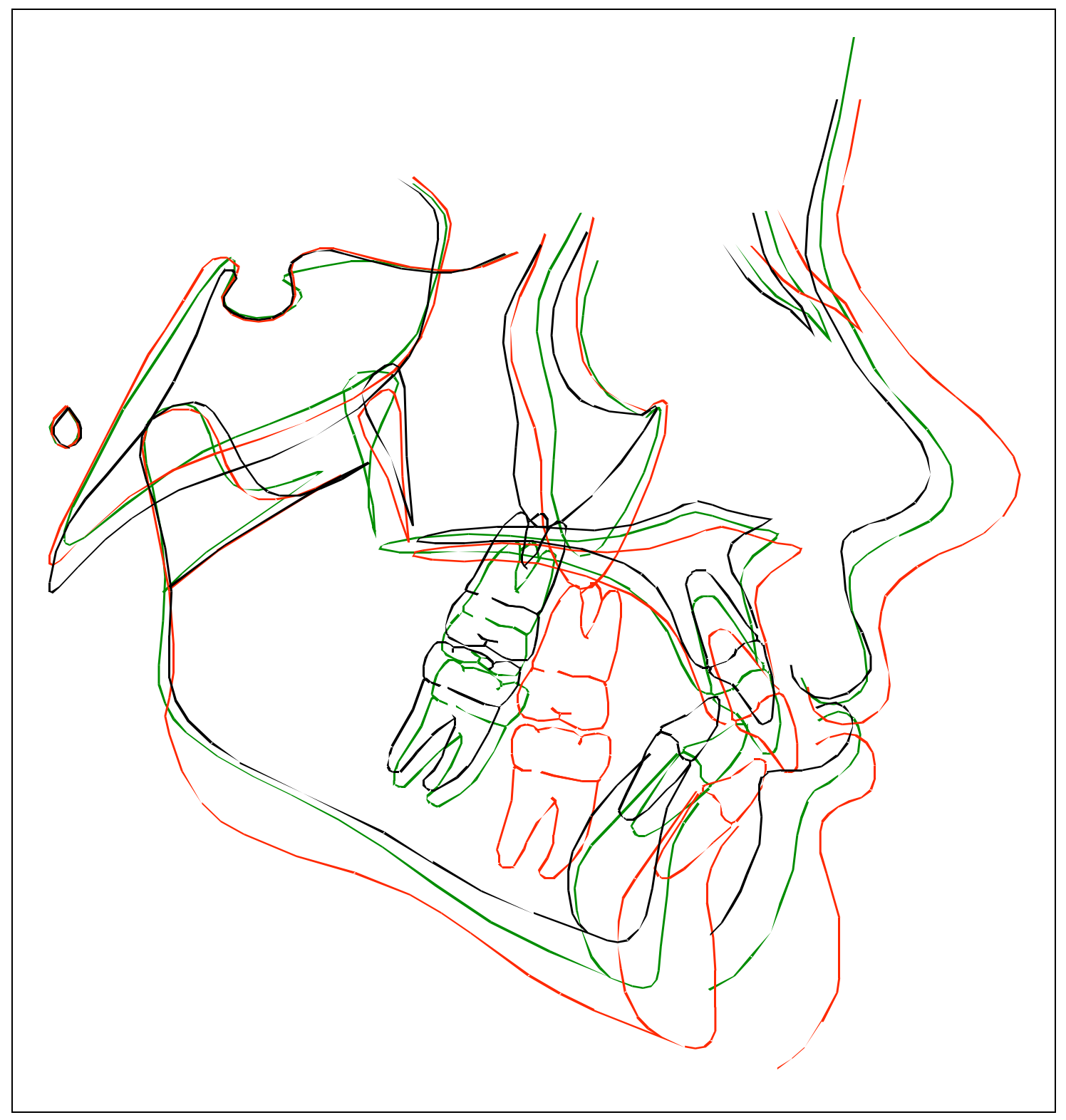

Figure A-41: Print of cephalometric tracing superimposition subject 41 . 


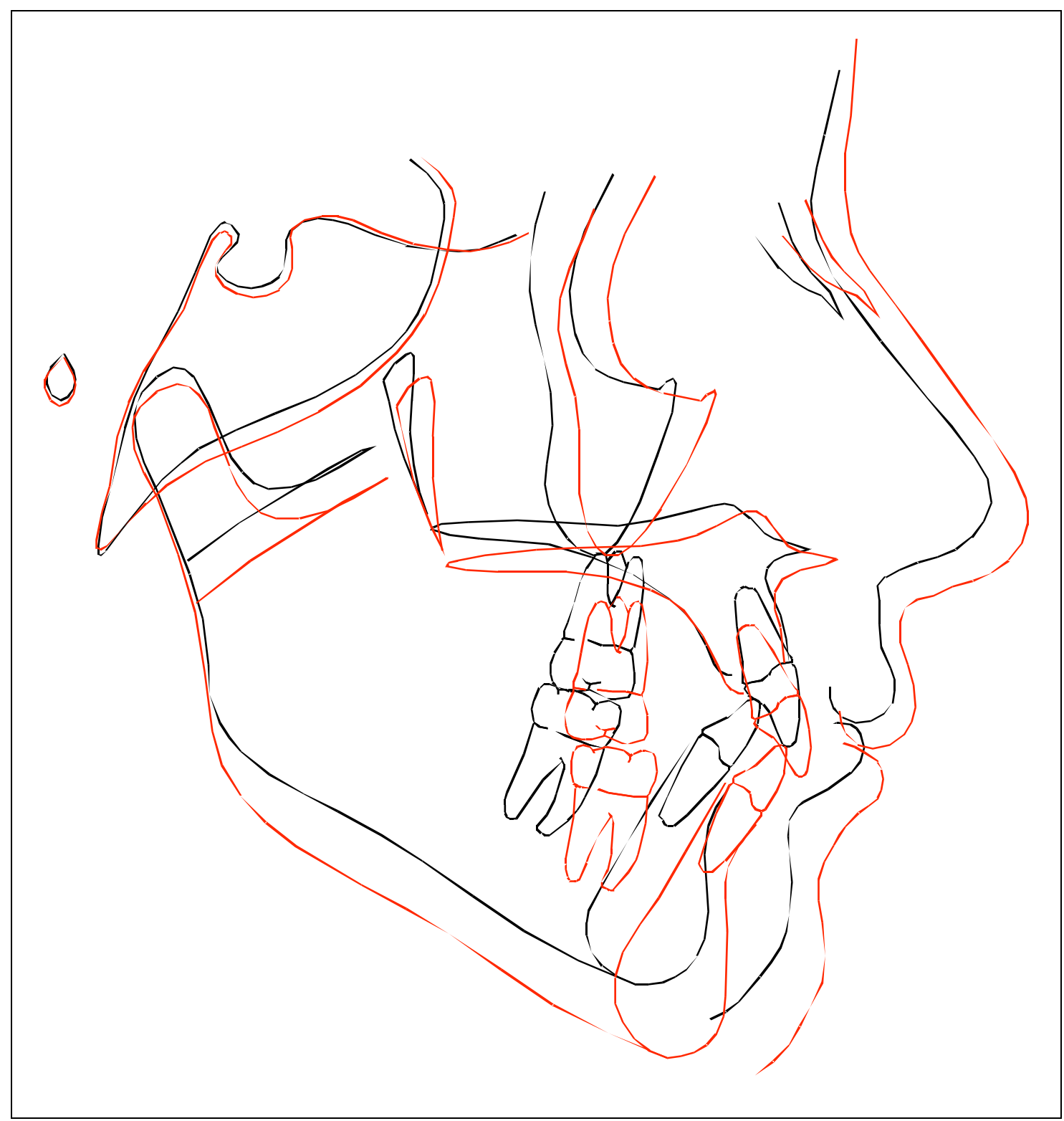

Figure A-42: Print of cephalometric tracing superimposition subject 42 . 


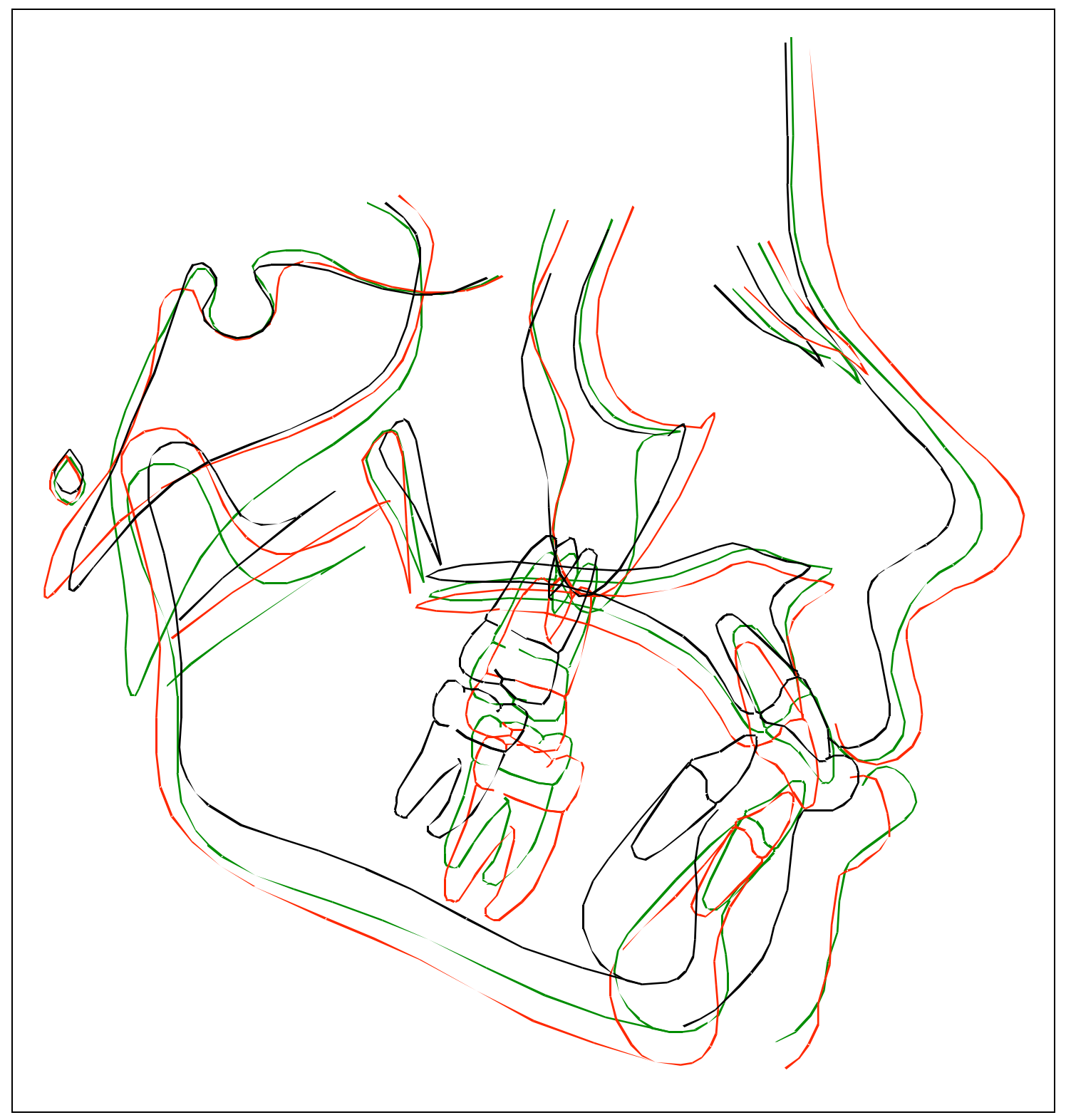

Figure A-43: Print of cephalometric tracing superimposition subject 43 . 


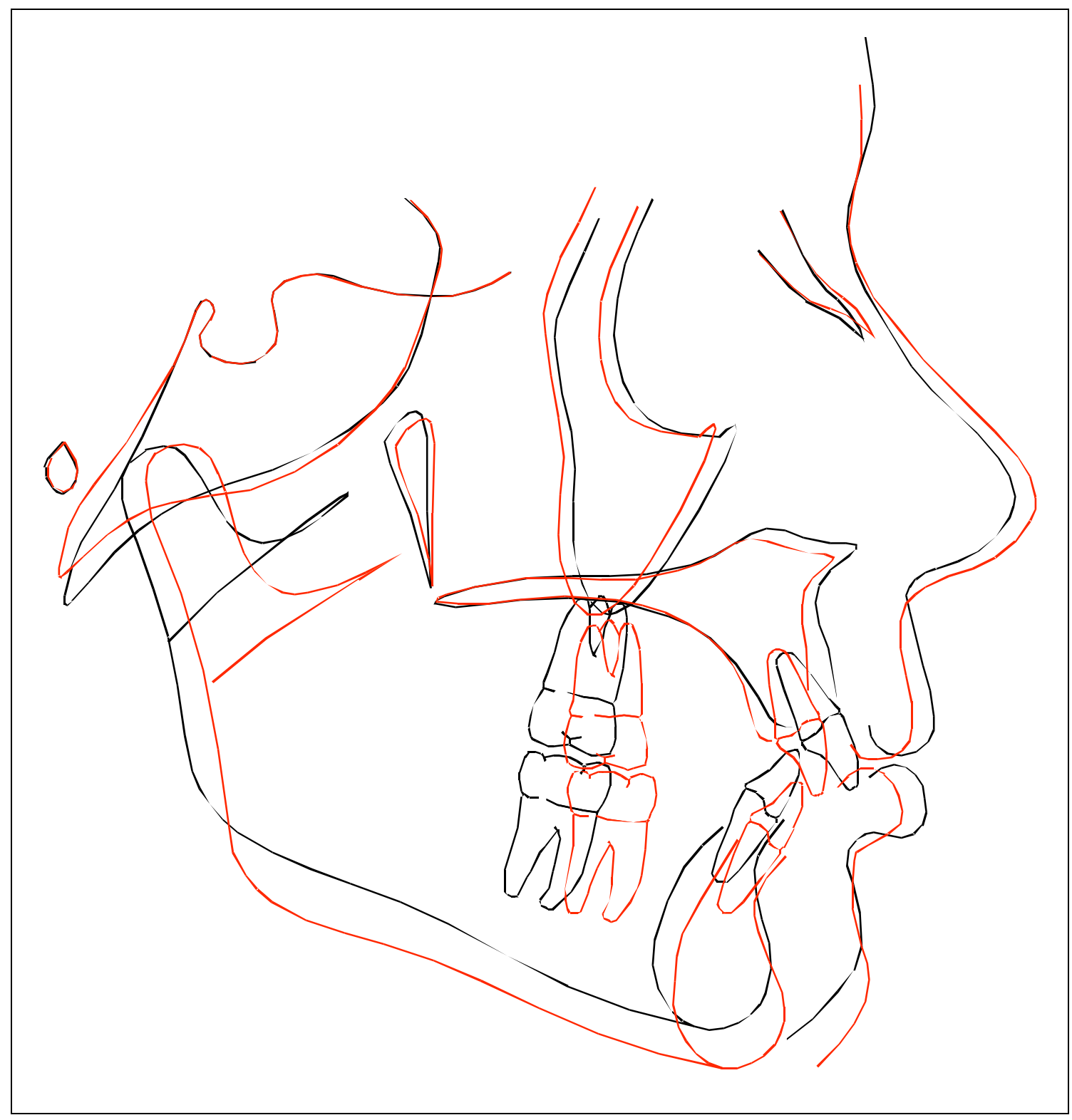

Figure A-44: Print of cephalometric tracing superimposition subject 44 . 


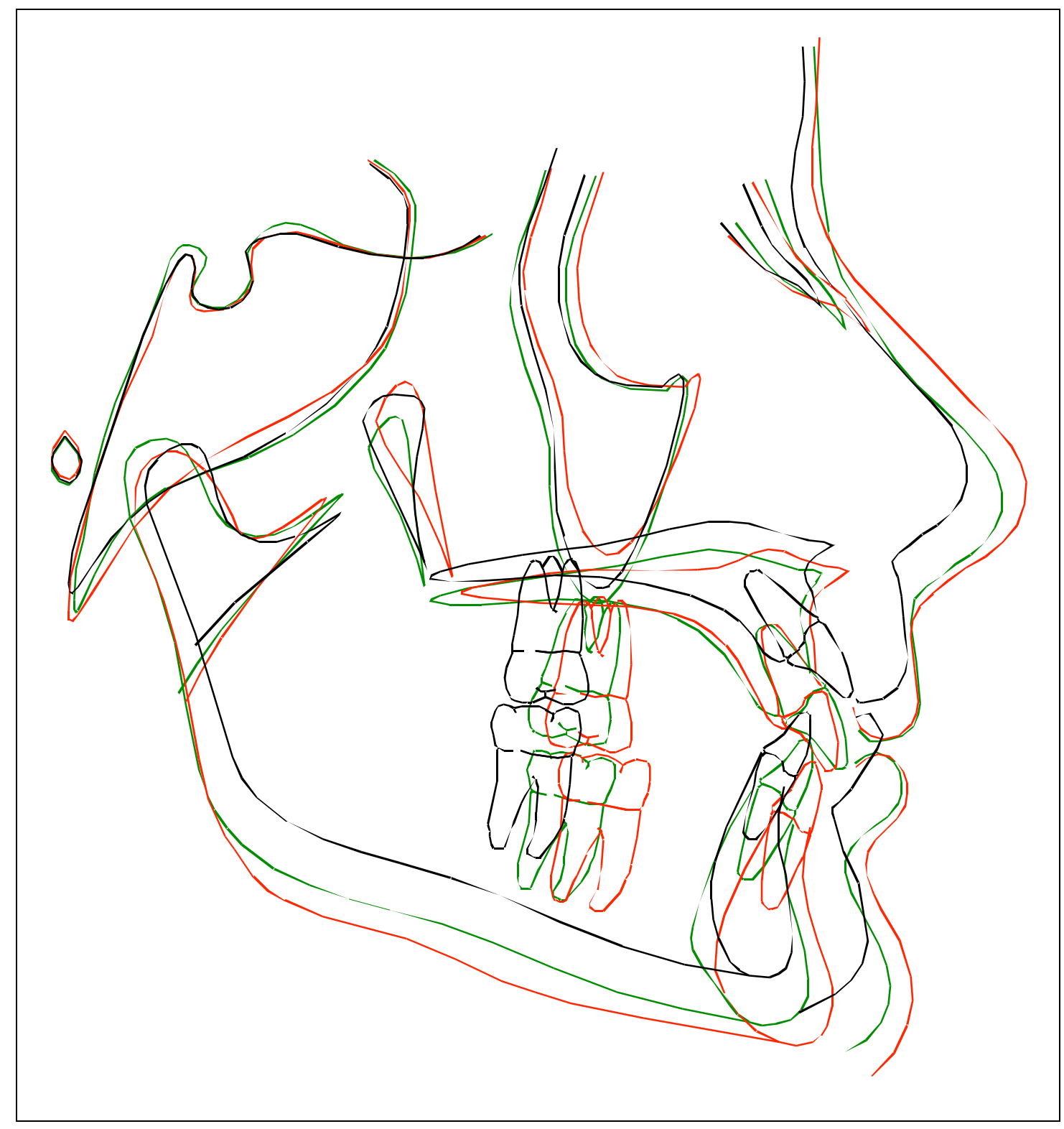

Figure A-45: Print of cephalometric tracing superimposition subject 45 . 


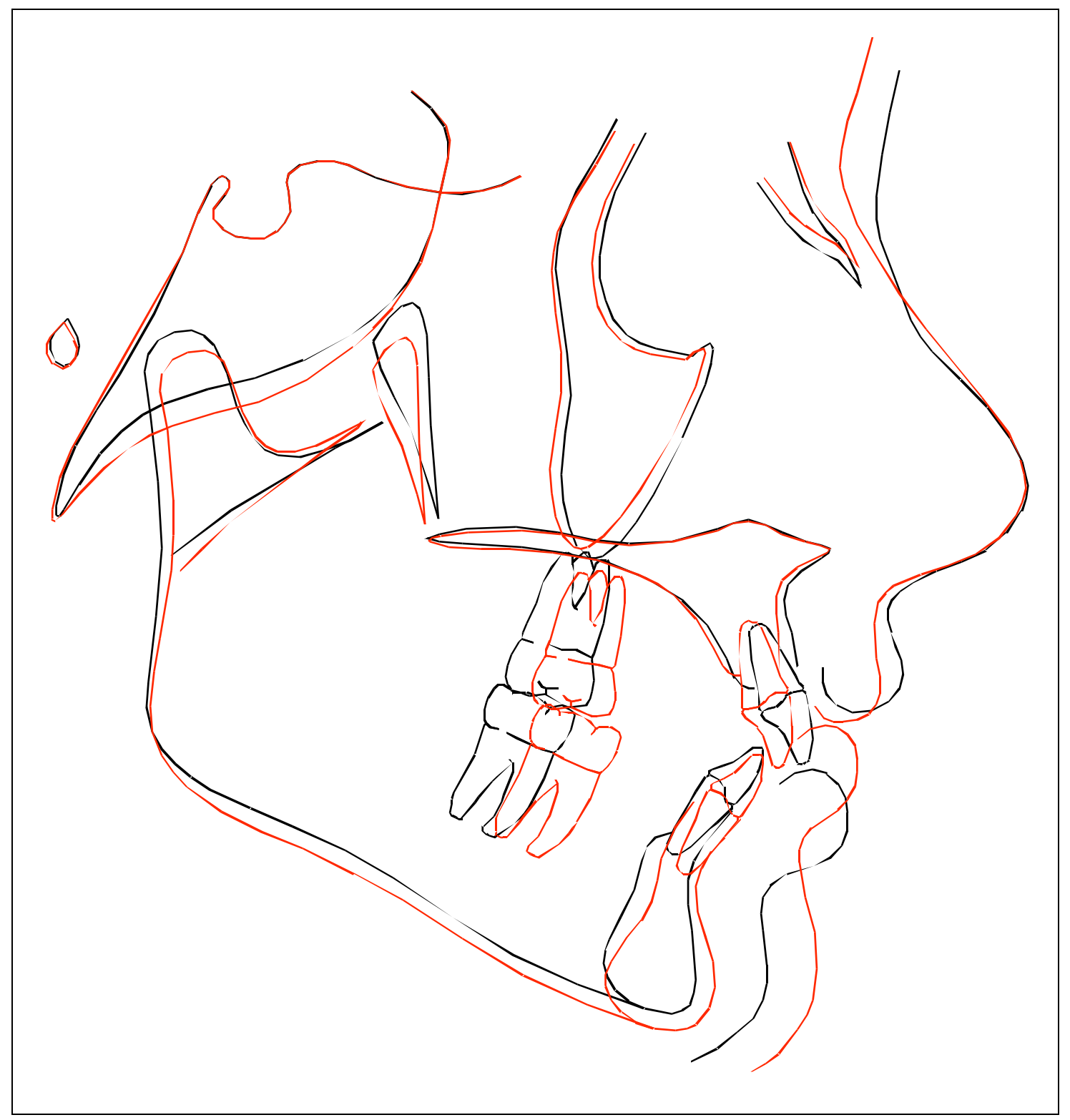

Figure A-46: Print of cephalometric tracing superimposition subject 46. 


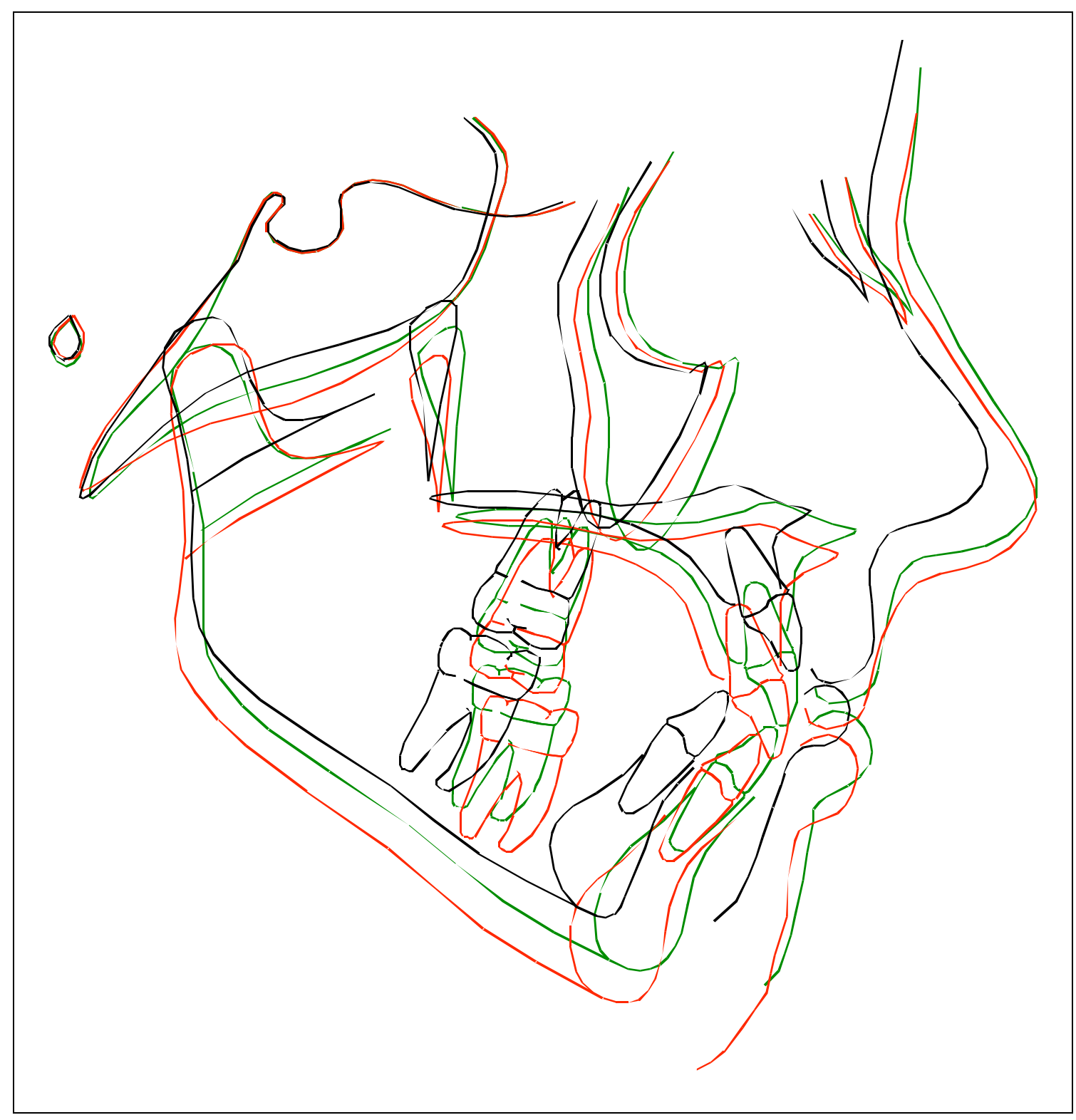

Figure A-47: Print of cephalometric tracing superimposition subject 47 . 


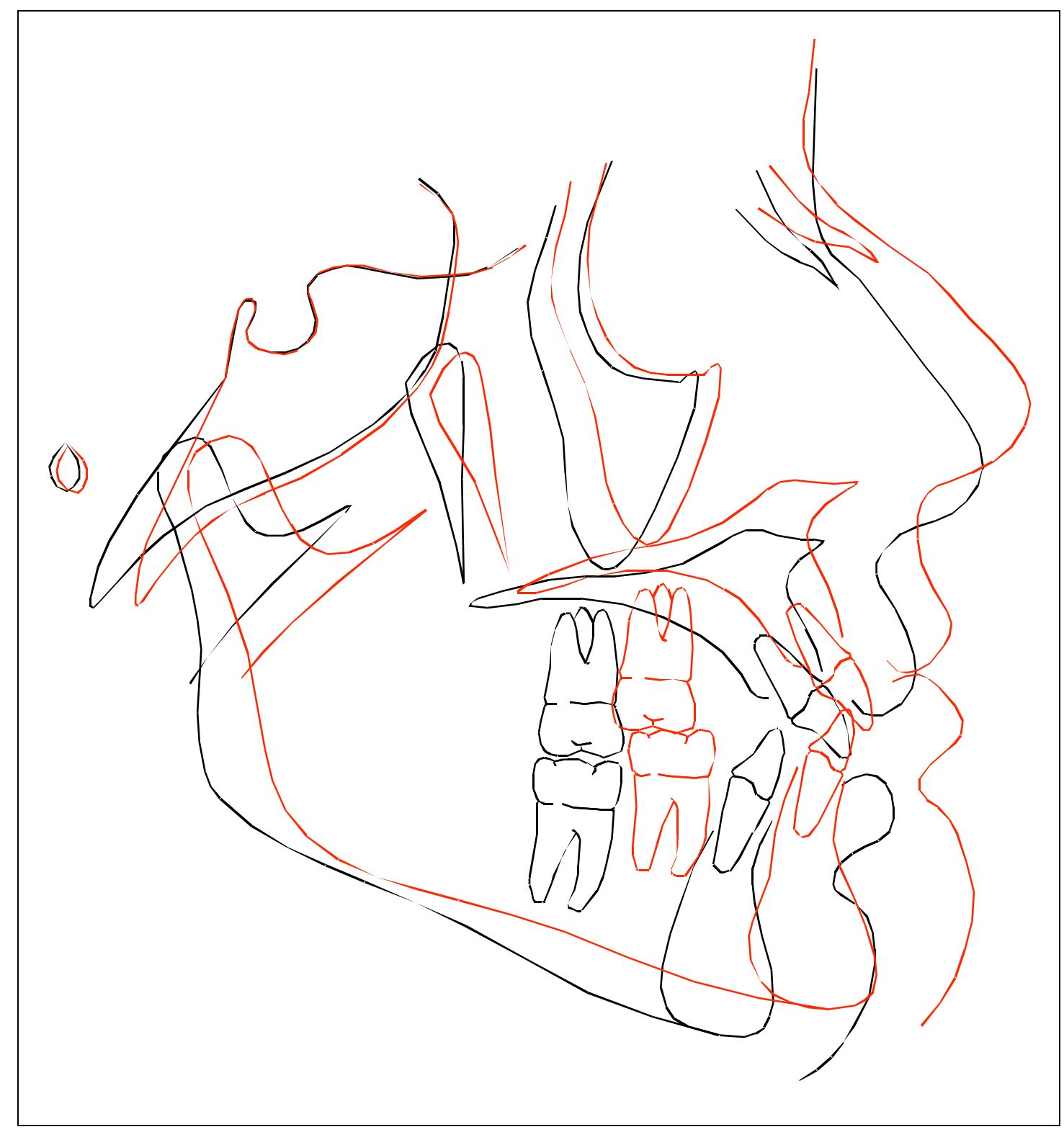

Figure A-48: Print of cephalometric tracing superimposition subject 48 . 


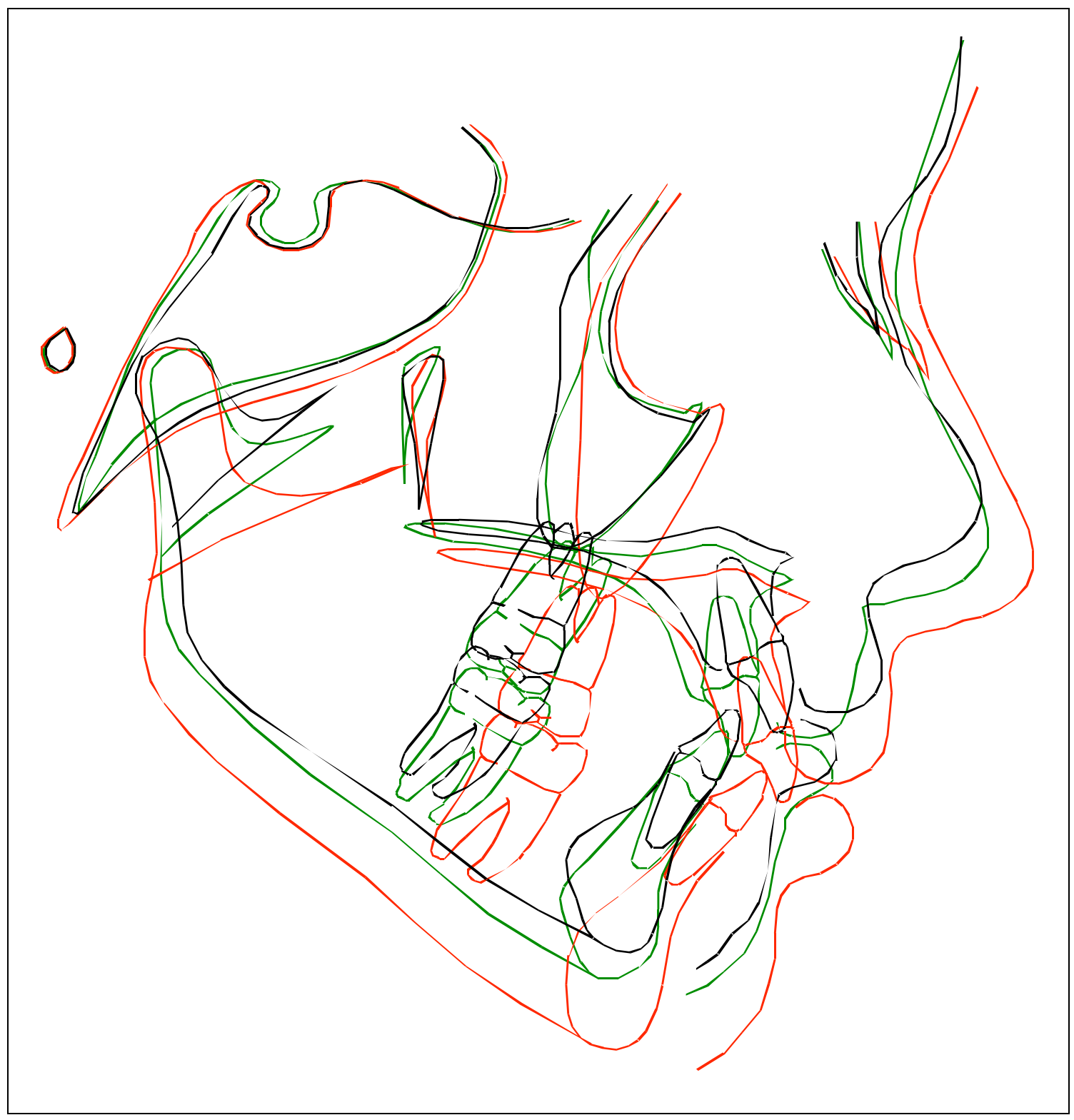

Figure A-49: Print of cephalometric tracing superimposition subject 49. 


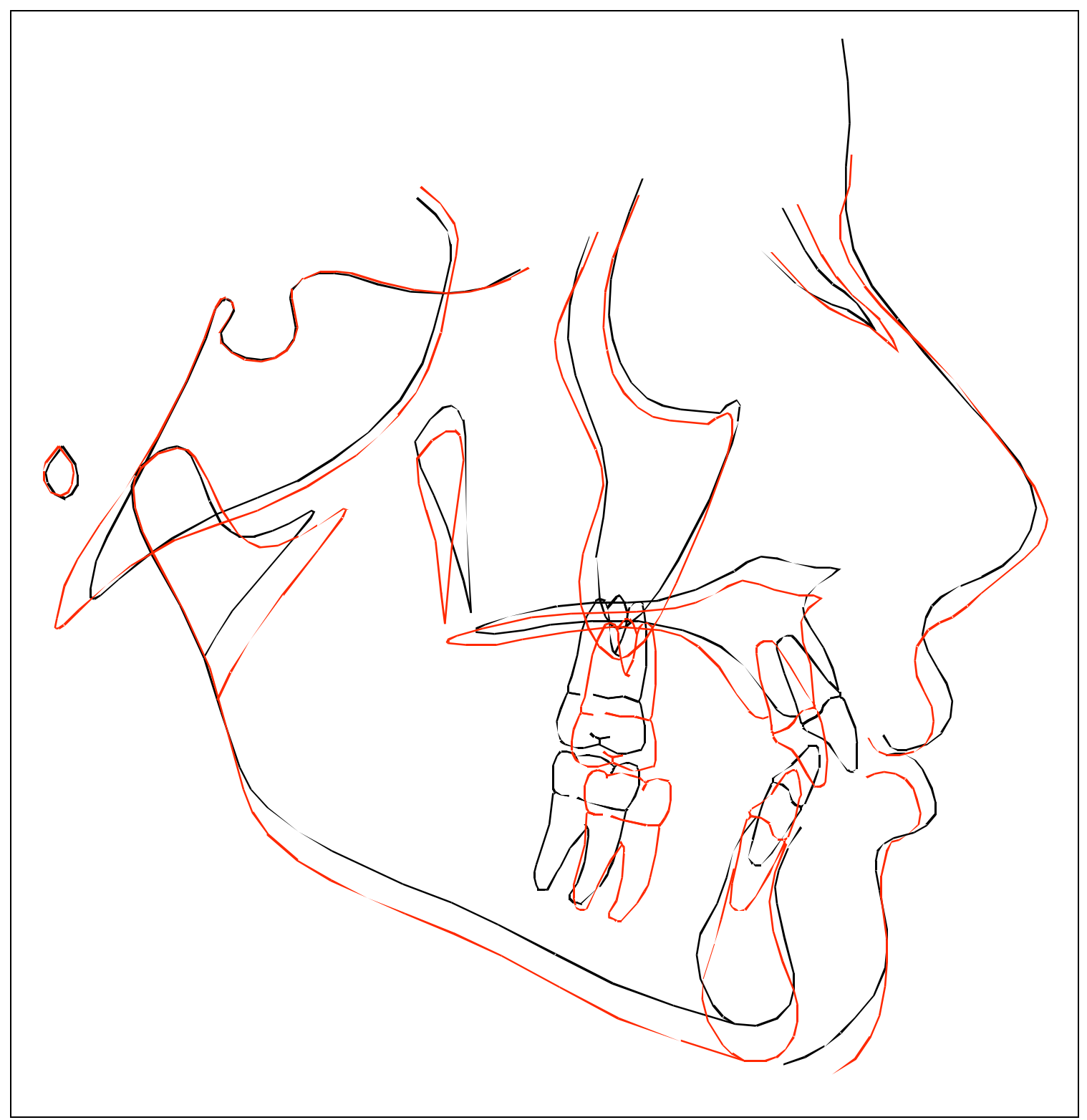

Figure A-50: Print of cephalometric tracing superimposition subject 50. 


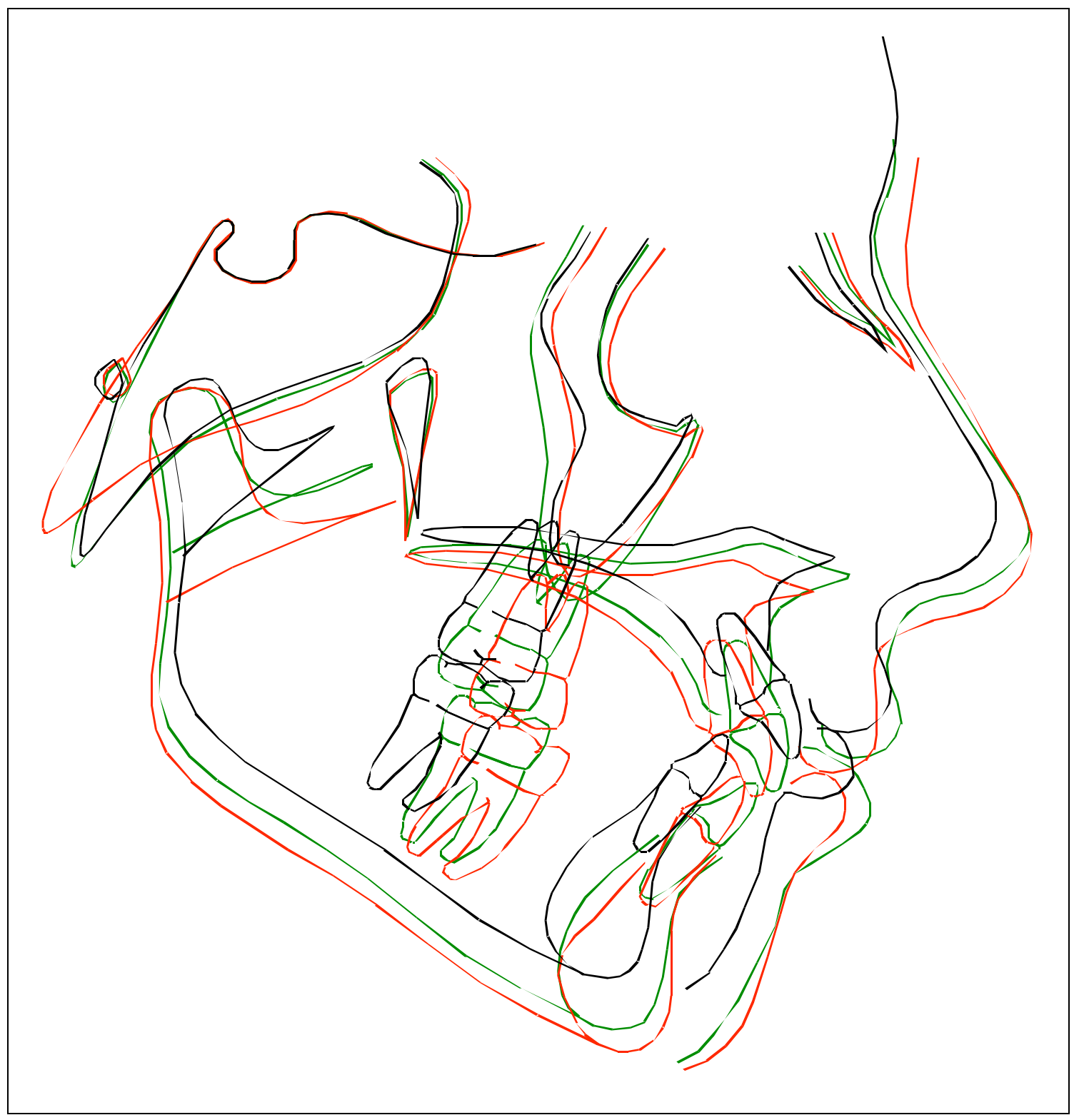

Figure A-51: Print of cephalometric tracing superimposition subject 51. 


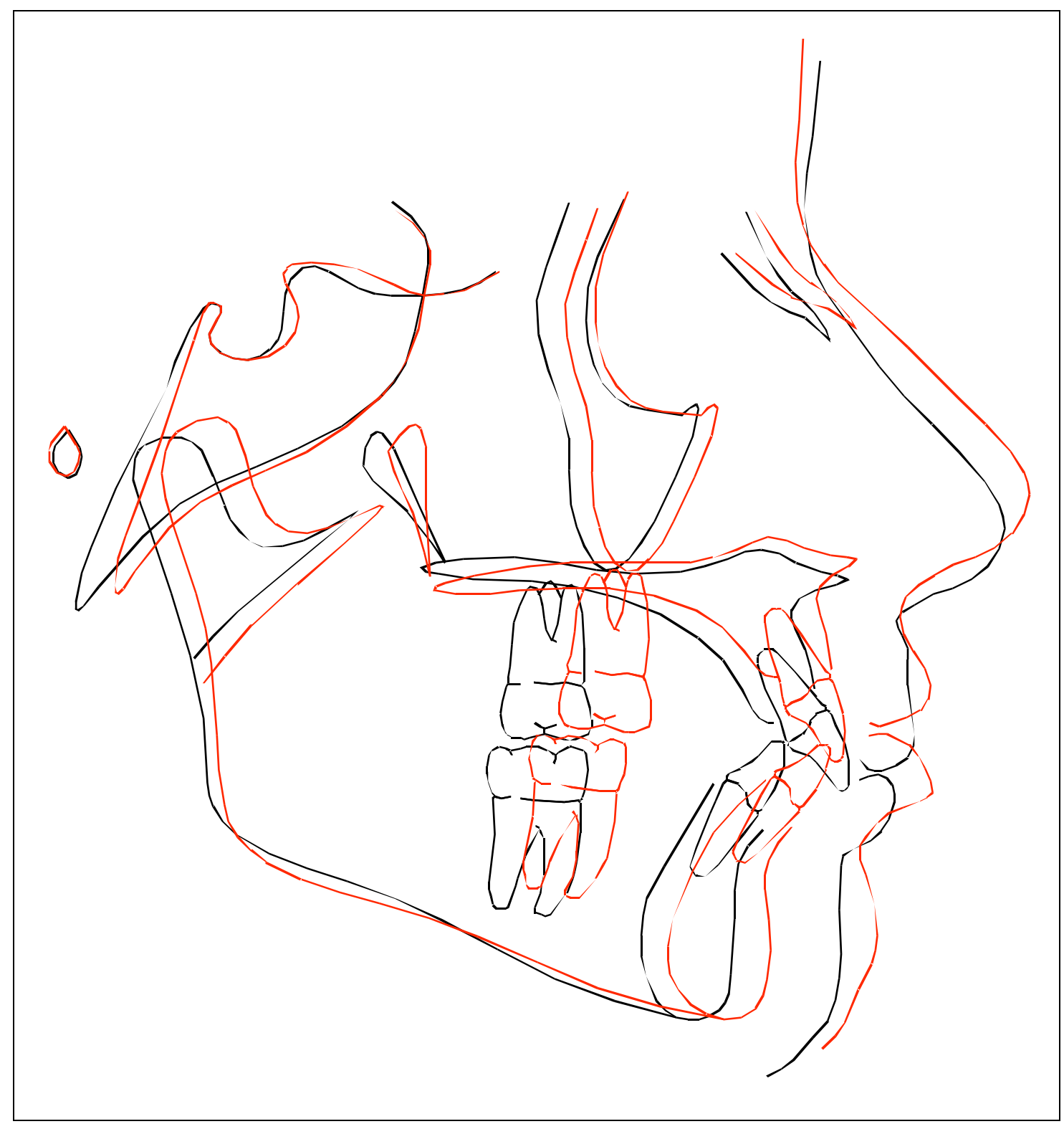

Figure A-52: Print of cephalometric tracing superimposition subject 52. 


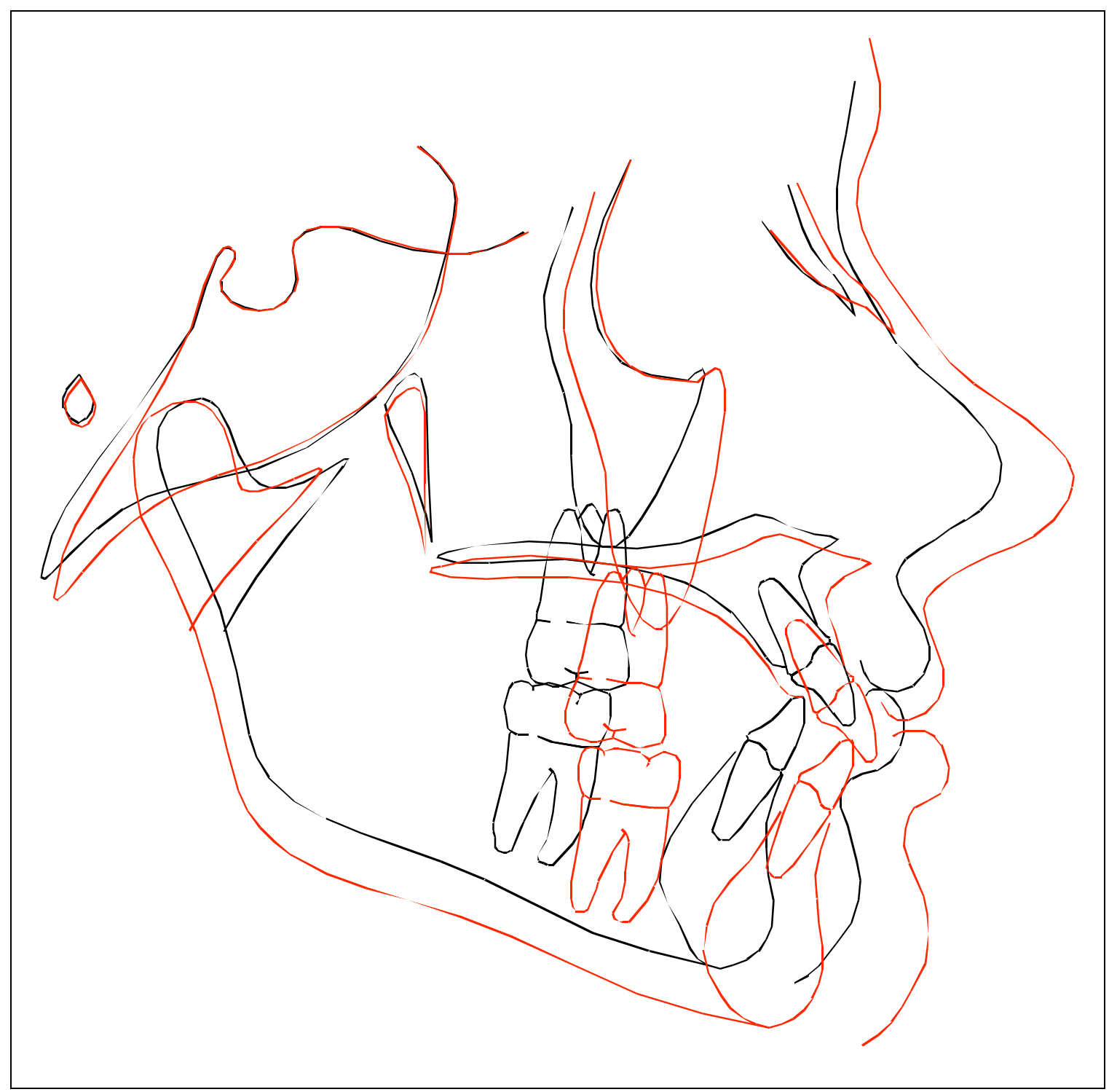

Figure A-53: Print of cephalometric tracing superimposition subject 53. 


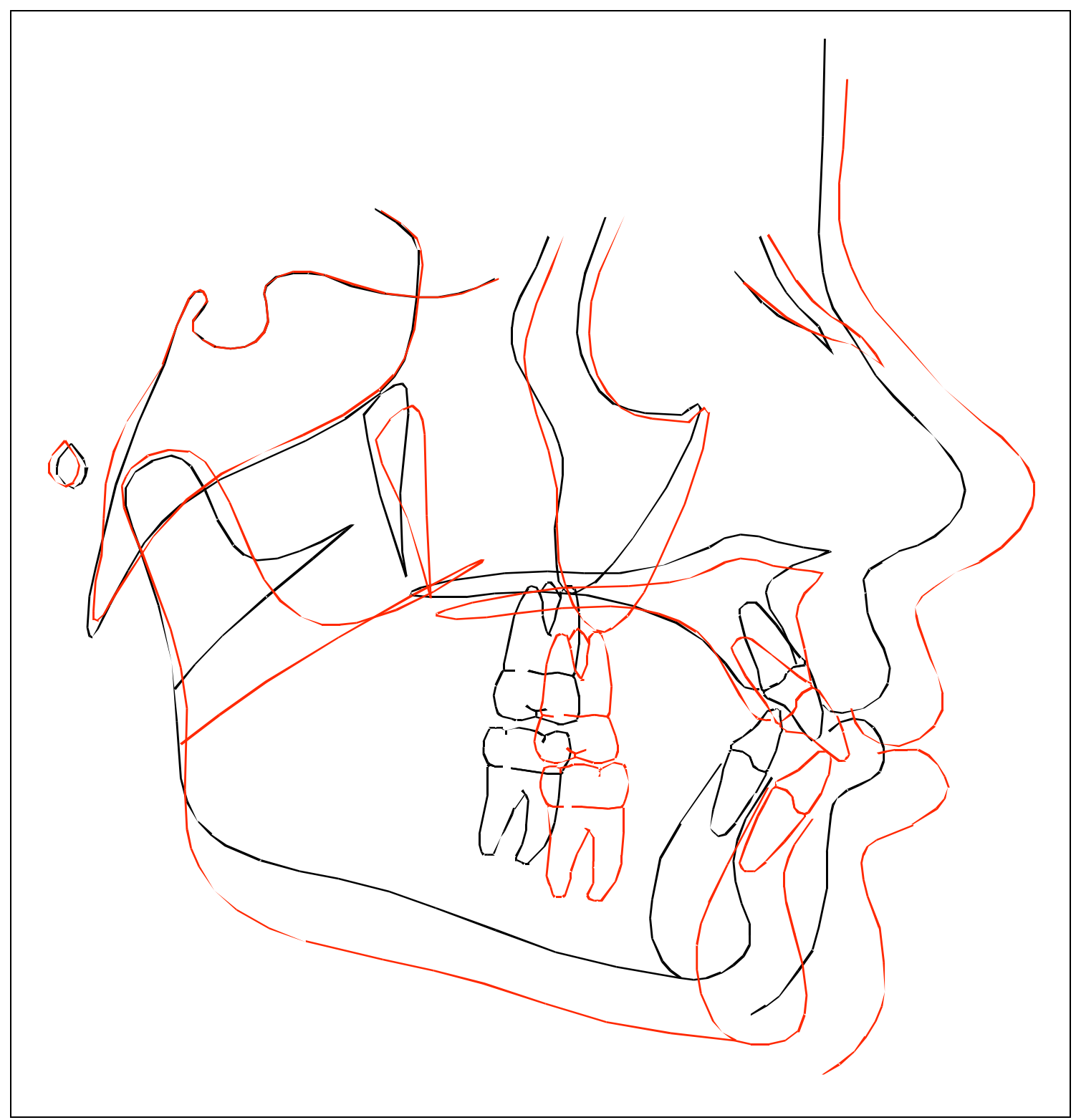

Figure A-54: Print of cephalometric tracing superimposition subject 54. 


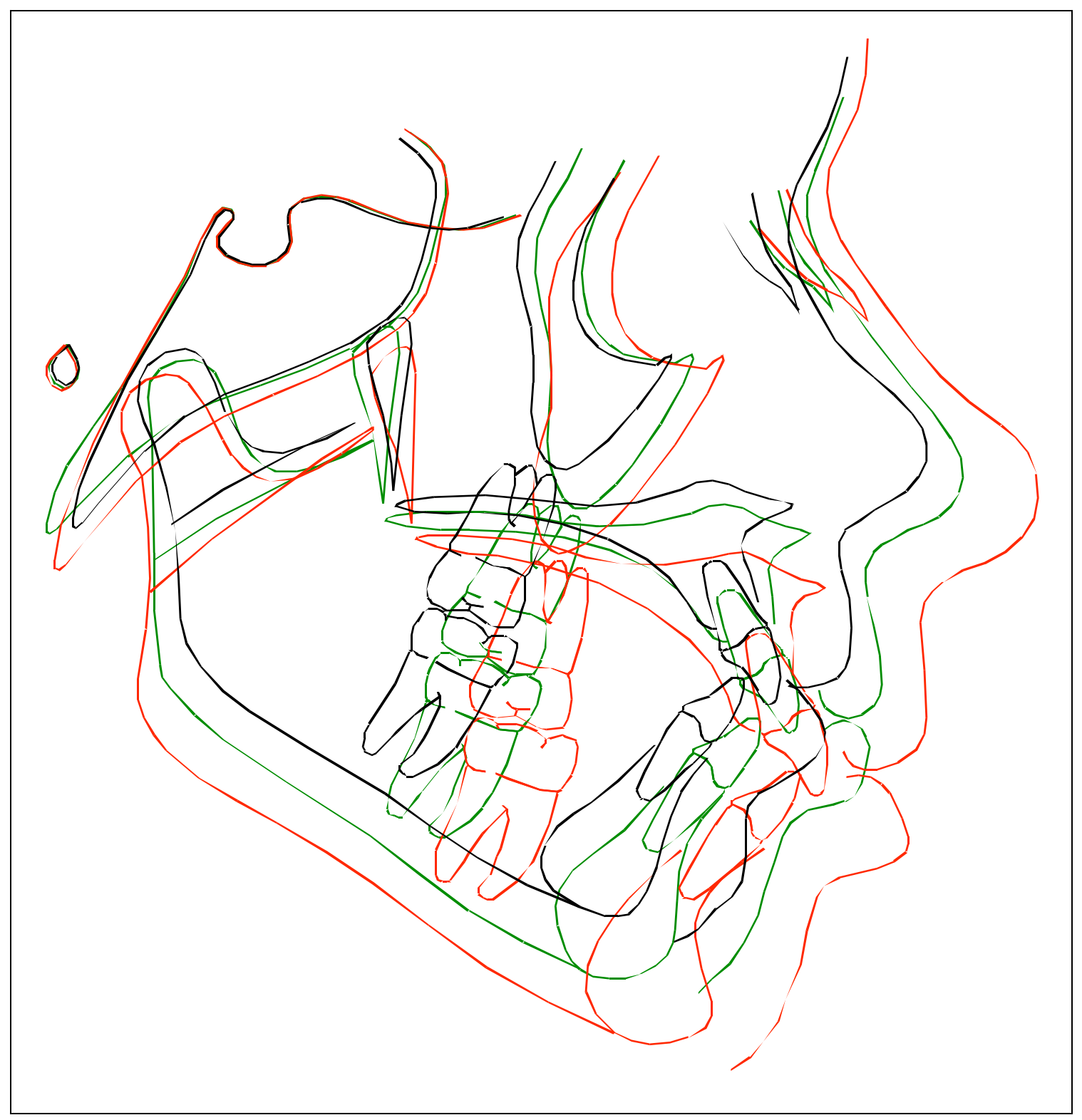

Figure A-55: Print of cephalometric tracing superimposition subject 55. 


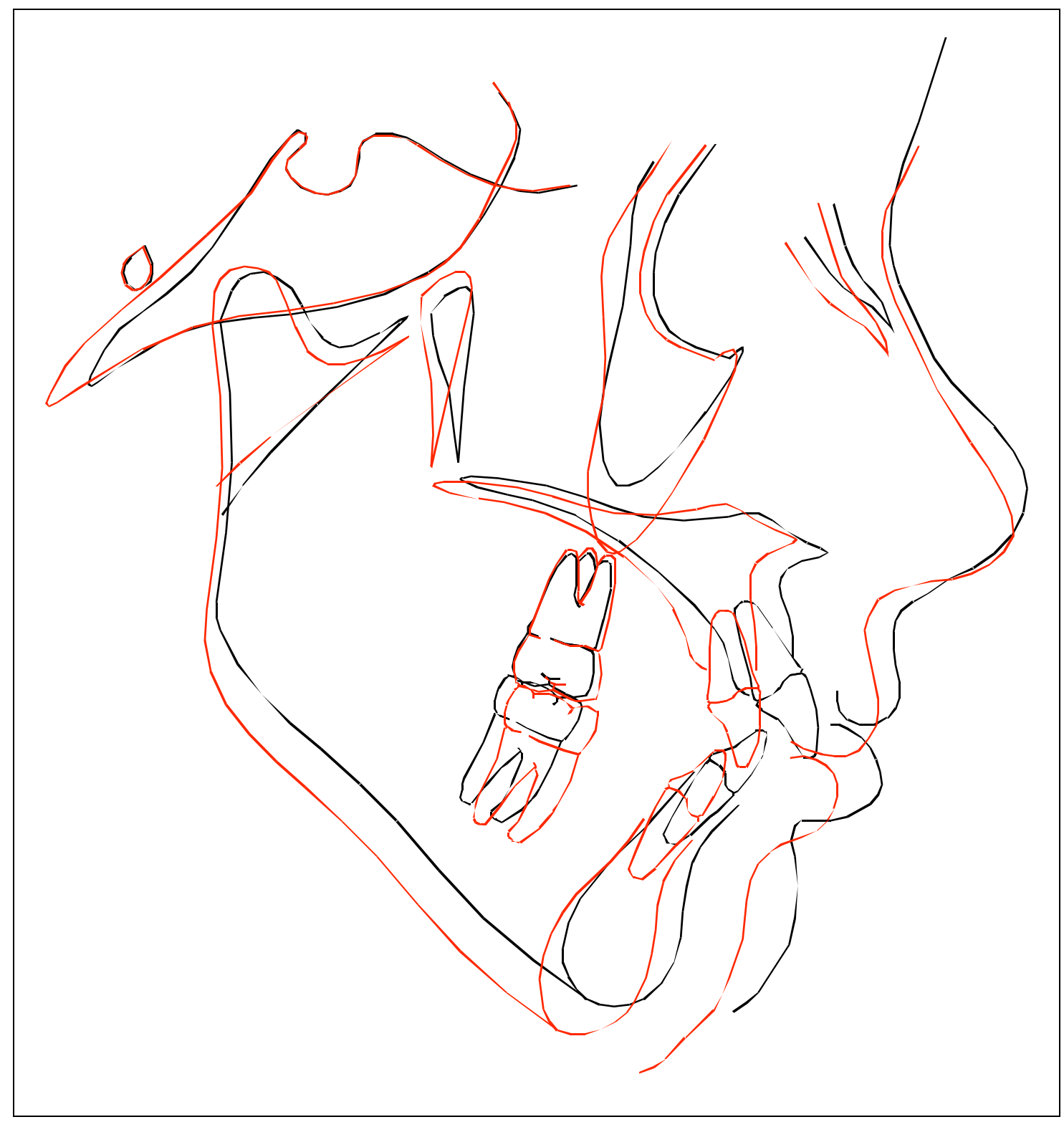

Figure A-56: Print of cephalometric tracing superimposition subject 56. 


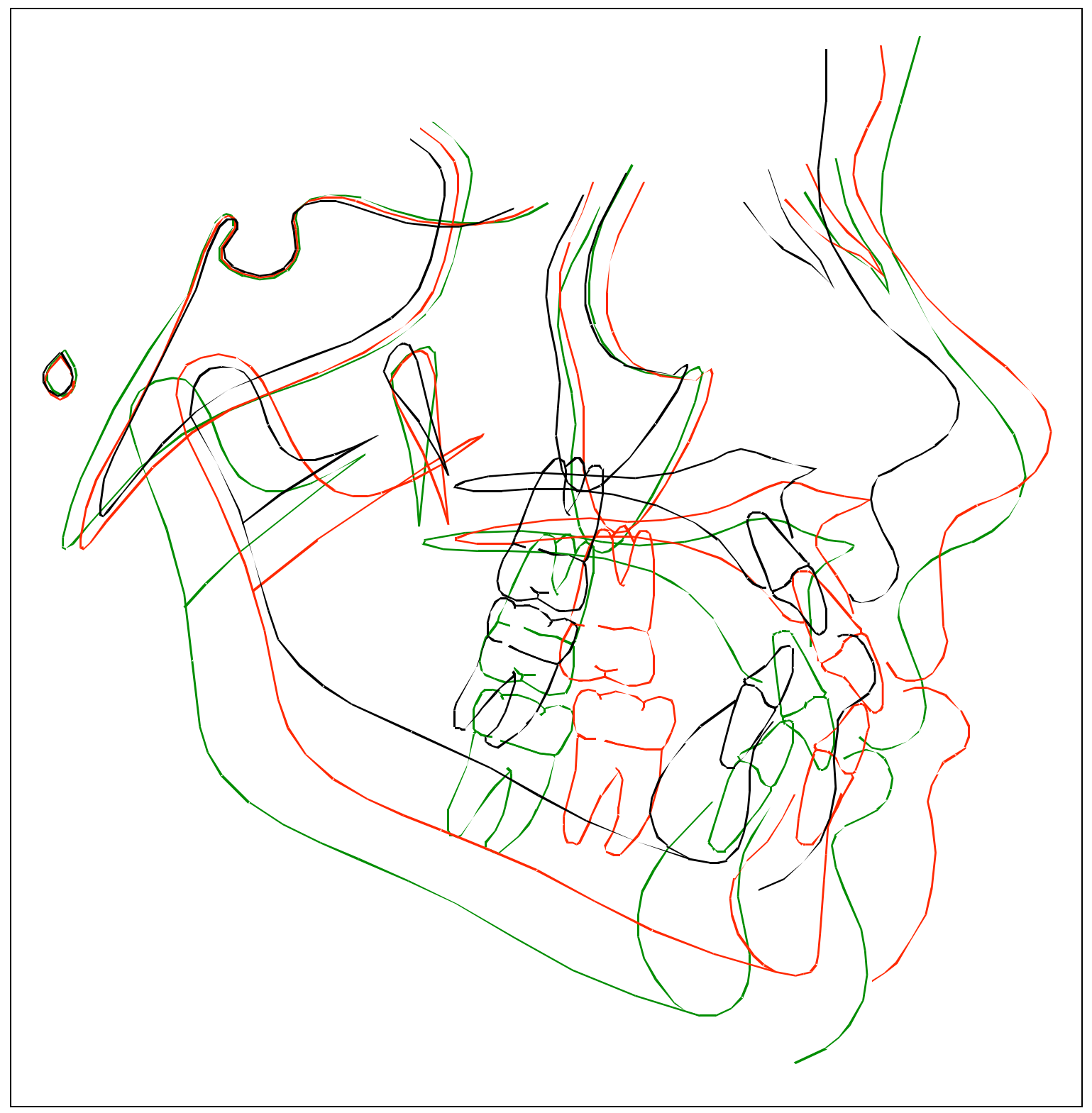

Figure A-57: Print of cephalometric tracing superimposition subject 57. 


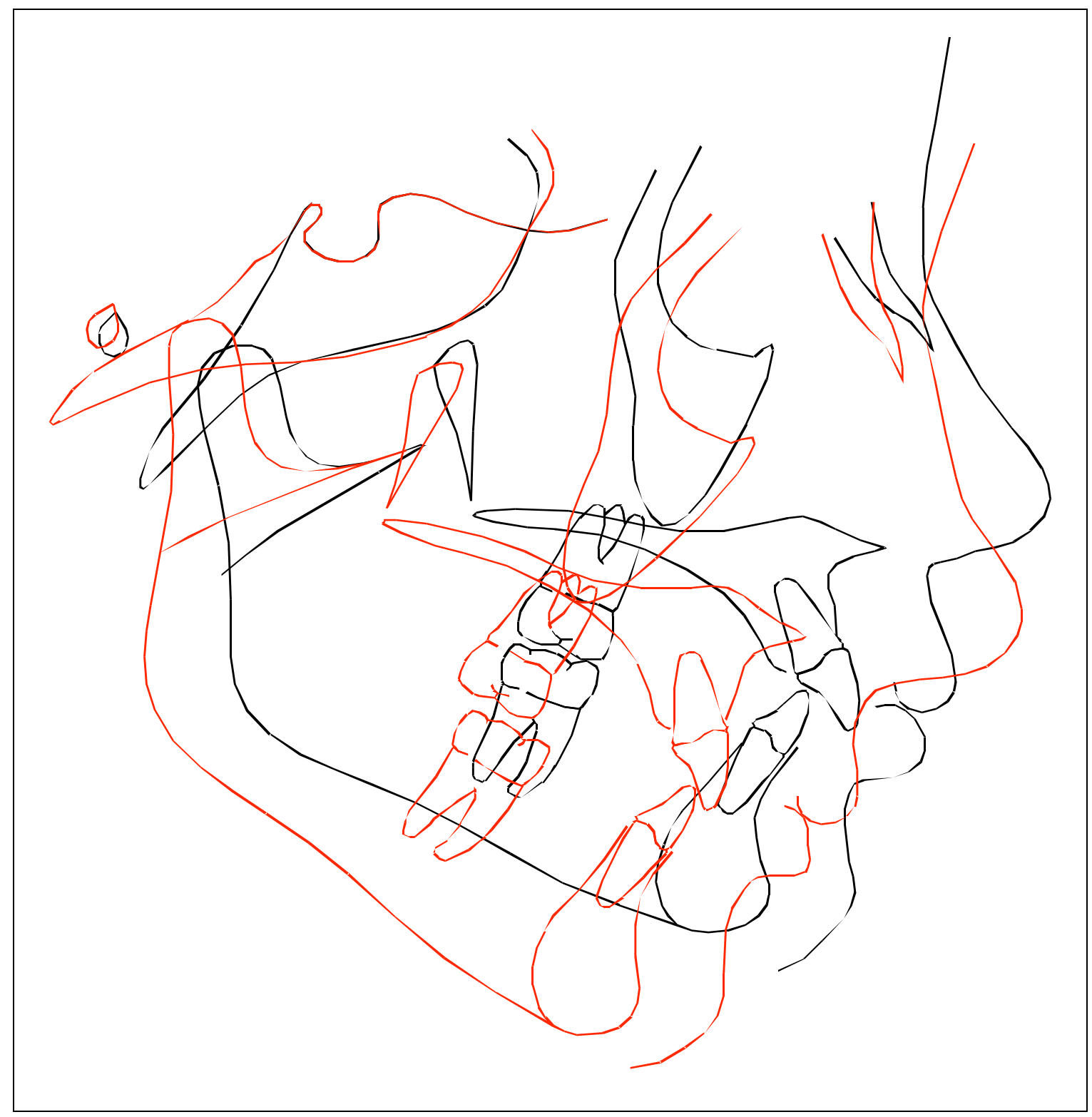

Figure A-58: Print of cephalometric tracing superimposition subject 58. 


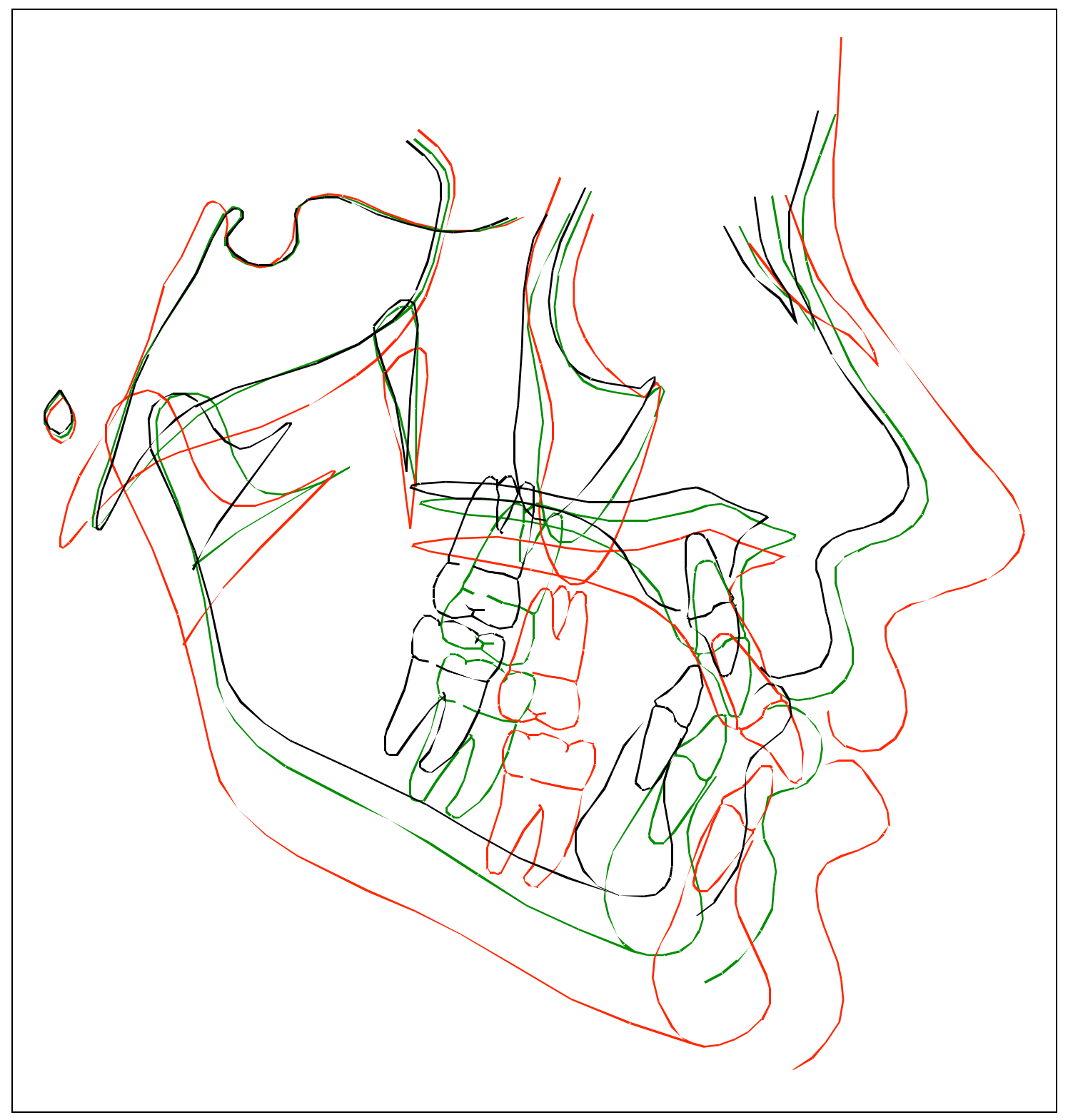

Figure A-59: Print of cephalometric tracing superimposition subject 59. 


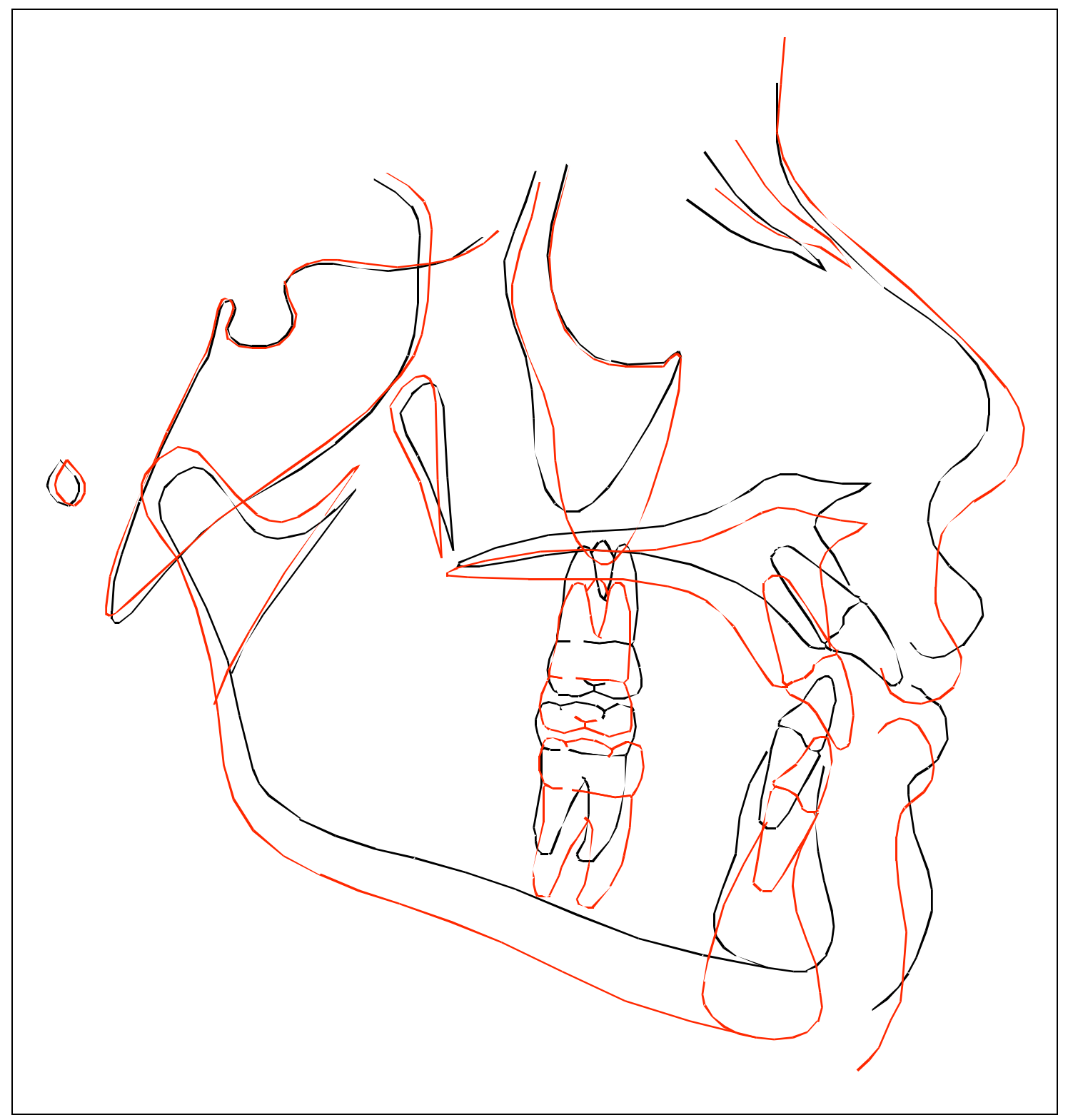

Figure A-60: Print of cephalometric tracing superimposition subject 60 . 


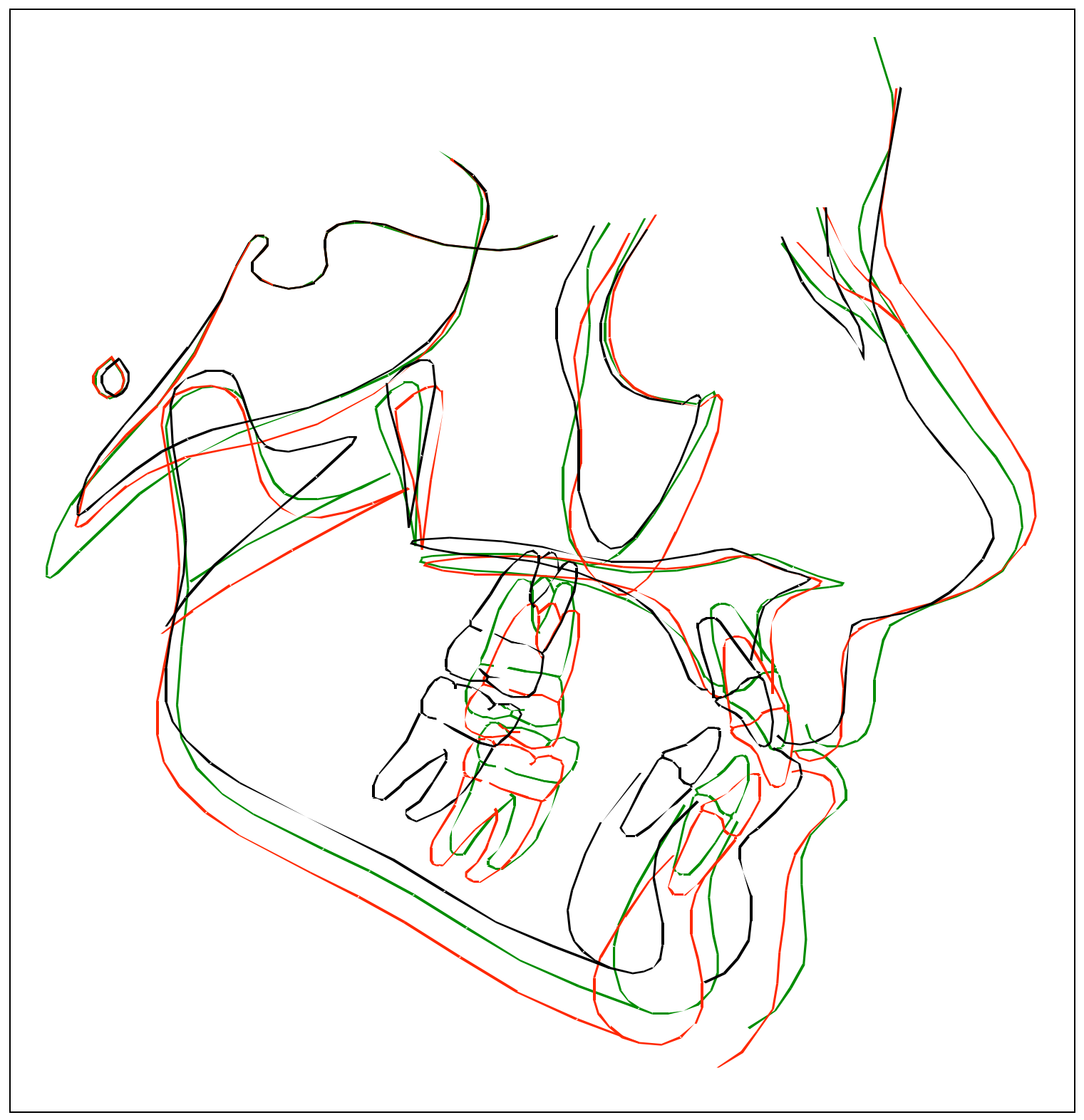

Figure A-61: Print of cephalometric tracing superimposition subject 61. 


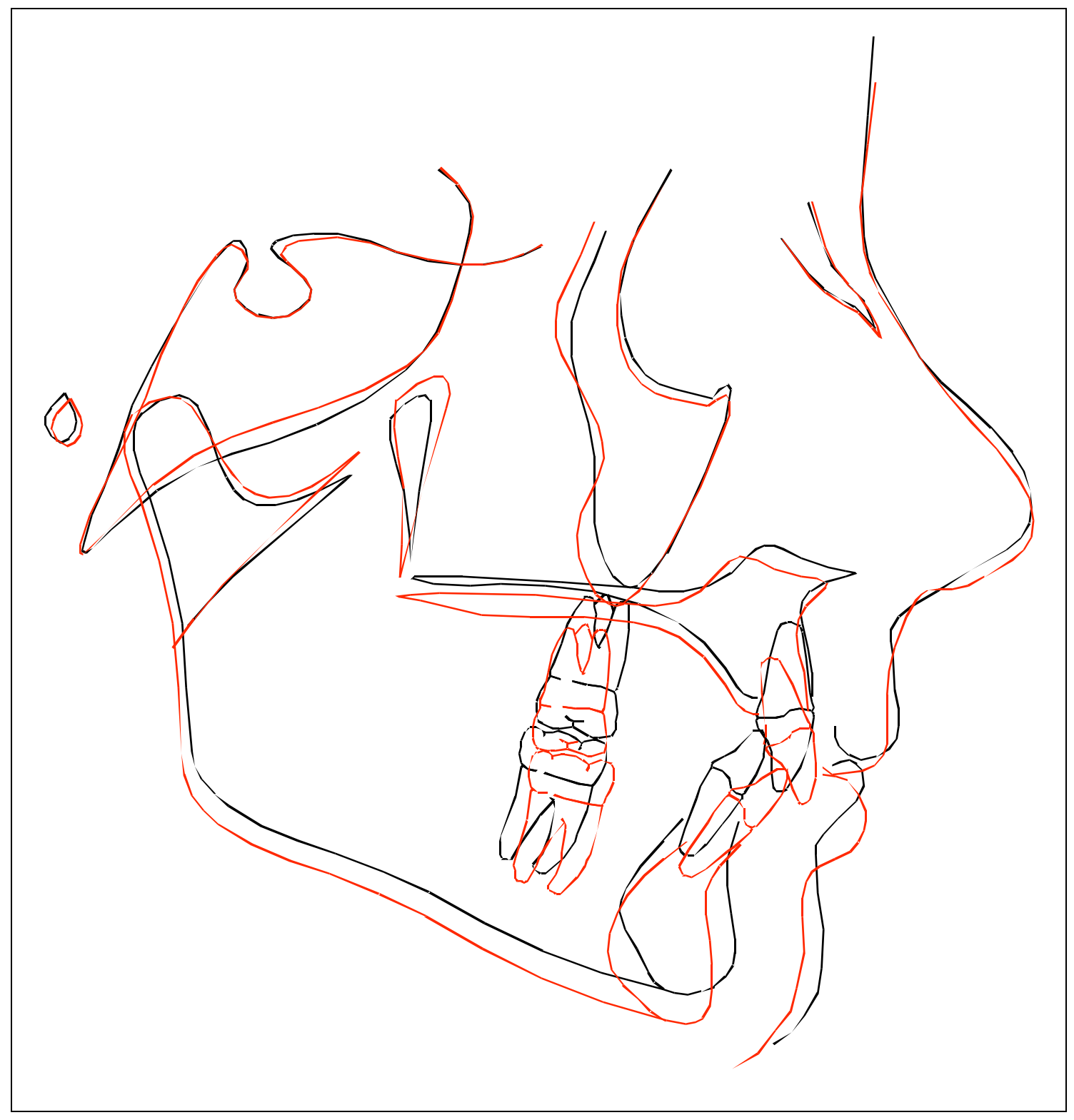

Figure A-62: Print of cephalometric tracing superimposition subject 62. 


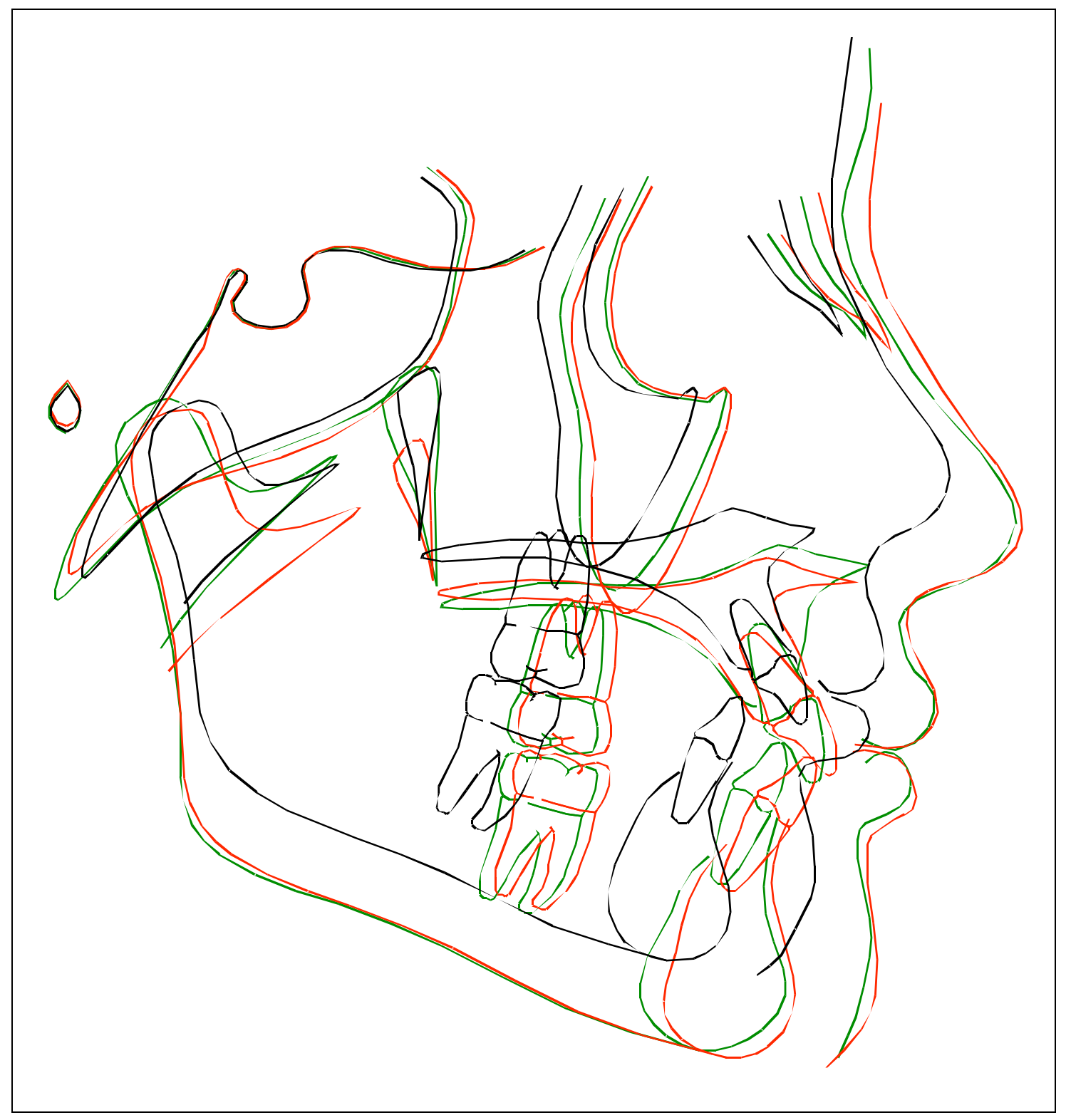

Figure A-63: Print of cephalometric tracing superimposition subject 63. 


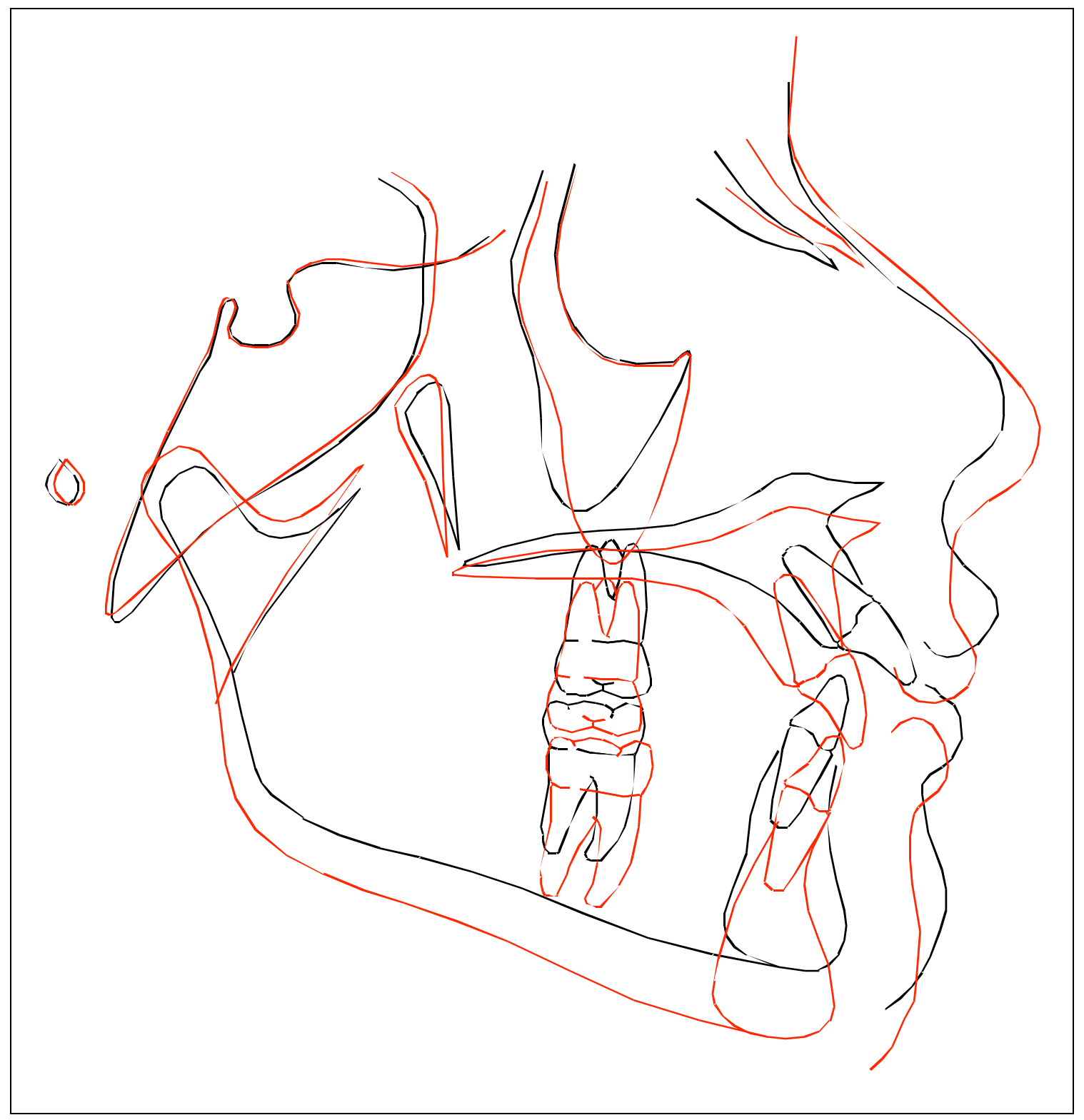

Figure A-64: Print of cephalometric tracing superimposition subject 64 . 
Frederick Jerome Burr was born in Nashville, Tennessee, on January 13, 1977. Jerome graduated from Dickson County High School in 1995. Jerome attended The University of Tennessee, Knoxville, where he received a Bachelor of Science in Biology and graduated with honors. After The University of Tennessee, Knoxville, he attended The University of Tennessee, Memphis School of Dentistry and was awarded the Doctor of Dental Surgery degree in May 2004. In August 2004, he entered The University of Tennessee as a graduate student in the Department of Orthodontics and is expected to receive his Master of Dental Science in May 2007. Jerome, his wife Amanda, and their son Elliott plan to live in Dickson, Tennessee, upon graduation. 